\title{
A Joint DOE/NRC Field Study of Tracer Migration in the Unsaturated Zone
}

\author{
John Nyhan \\ Will Polzer \\ Edward Essington \\ Edward Cokal \\ Leonard Lane \\ Edward Lopez \\ Ellen Stallings \\ Robin Walker
}

DE86 007861

\section{DISCLAIMER}

This report was prepared as an account of work sponsored by an agency of the United States Government. Neither the United States Government nor any agency thereof, nor any of their employees, makes any warranty, express or implied, or assumes any legal liability or responsibility for the accuracy, completeness, or usefulness of any information, apparatus, product, or process disclosed, or represents that its use would not infringe privately owned rights. Reference herein to any specific commercial product, process, or service by trade name, trademark, manufacturer, or otherwise does not necessarily constitute or imply its endorsement, recommendation, or favoring by the United States Government or any agency thereof. The views and opinions of authors expressed herein do not necessarily state or reflect those of the United States Government or any agency thereof. 
A JOINT DOE/NRC FIELD STUDY OF TRACER MIGRATION

IN THE UNSATURATED ZONE

by

John Nyhan, Will Polzer, Edward Essington, Edward Cokai, Leonard Lane, Edward Lopez, Ellen Stallings, and Robin Walker

\section{ABSTRACT}

The results of a joint DOE/NRC field experiment to evaluate leaching and transport of solutes in a sandy silt backfill used for shallow land burial operations at Los Alamos are presented for steady-state and unsteady-state flow conditions. The migration of iodide, bromide, and lithium through the backtill material is studied as functions of deptri and time and they are compared with one another.

The bromide and iodide tracer data are used to estimate the diffusion coefficient, the tortuosity factor, and dispersivity. These values are used to calculate effective dispersion coefficients for subsequent analyses of the retardation factor and the distribution coefficient for lithium using least squares procedures.

The results of the tracer inigration study are discussed relative to challenges facing the waste management community, and chemical transport modeling opportunities are presented for a modeling workshop to be held in FY86.

\section{INTRODUCTION}

The total volume of low-level radioactive wastes produced in the United States is conservatively projected to be about $16 \mathrm{million}^{3}$ by the year 2020 (US Department of Energy 1982). This increasing production rate is of major concern because new and acceptable sites will be required for the disposal of these wastes. Logistics dictates that new burial sites will need to be selected in a wide range of environments throughout the US, and actual or anticipated problems witn closed shallow land burial sites must also be corrected. 
The most popular current method for disposing of low-level radioactive wastes is shallow land burial (SLB). Burial trenches range in size from the 4.6-m deep, 3- by 15-m disposal pit at Oak Ridge National Laboratory to the 6.1-m deep, 30- by 300-m trench at Barnwel1, South Carolina. After waste materials are placed in these trenches, current management practices range from simple backfilling of the trench to more elaborate installation of multilayered trench caps and revegetation programs.

Once the burial trench receives its final cover, several environmental processes begin to influence the configuration and integrity of the surface and subsurface of the trench cap (Fig. 1). The most serious problems encountered in shallow land burial are related to water management (Jacobs et a1. 1980). As water comes into contact with the buried wastes, either from infiltration of precipitation or from trench cap erosion and subsidence, the integrity of the buried wastes is breached. Scientific planning for surface and subsurface water management at the SLB site requires a knowledge of the relationships between those factors that cause a loss of soil and water (Fig. 1), as well as a knowledge of the migration of water and contaminants in porous materials beneath the SLB trench cap.

The siting requirements described by the Nuclear Regulatory Commission (NRC) require disposal in unsaturated media above fluctuations in the water table, unless it can be conclusively demonstrated that site characteristics will result in molecular diffusion being the predominant mechanism of radionuclide migration (see 10 CFR, Part 61 in NRC 1982). Wastes buried in this unsaturated media may include spent reactor resins that have been solidified in cement and packaged in 55-gallon drums. Past burial operations did not require that the resins be solidified in cement. Another requirement 
in 10 CFR, Part 61 for the burial of waste is that the capability exists for the disposal area to be modeled.

Required disposal in unsaturated media ussumes that leaching and transport of contaminants is minimized under those conditions. That assumption has not been verified experimentally. In fact, under some circumstances, evidence suggests that leaching and transport may be enhanced. Biggar and Nielsen 1962 suggest that scils at a water content below saturation could be more efficiently leached and thereby reduce the amount of water required in removing (transporting) undesirable salt concentrations. Leaching uy rainfall was considered to be more efficient because it occurs under unsaturated conditions. Unsaturated flow affects the manner in which pore water velocity and diffusion contribute to the mixing process in a porous media.

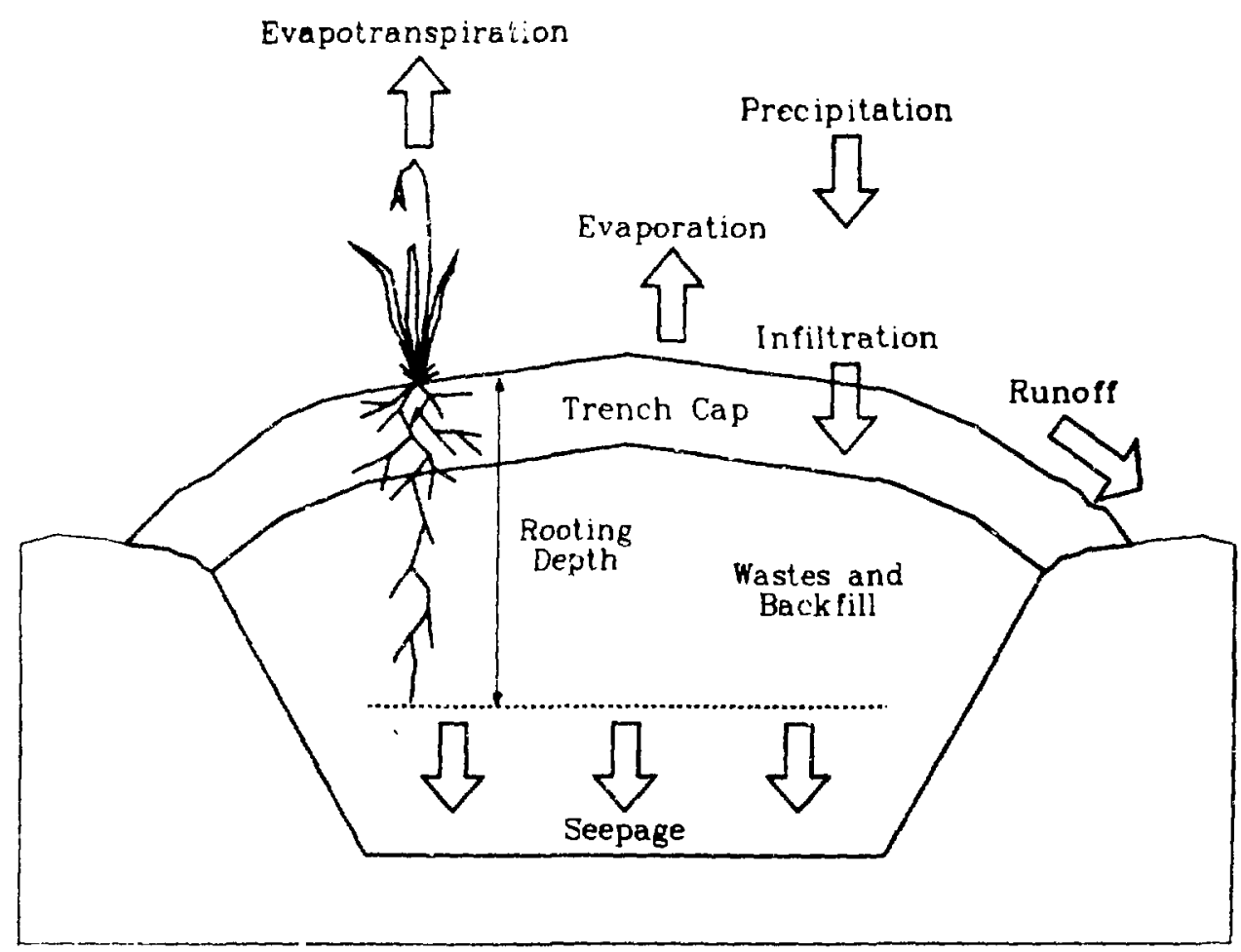

Fig. 1. Hydrology of shallow land burial. 
Wilson and Luthin 1963 observed that rainfall leached salts from a diatomaceous earth soil more efficiently than larger quantities of water applied by ponding. Nielsen et al. 1965 observed similar results when leaching by ponding was compared to internitient sprinkling. Miller et al. 1965 fourd $a$ difference in the redistribution of chloride where water was applied by two different inethods, continuous ponding and intermittent applications. They concluded that leaching was more efficient under unsaturated conditions and slow flow rates.

Brookhaven National Laboratory conducted laboratory experiments to investigate the influence of a wet-dry leaching cycle on the removal of radionuclides from solidified resin-cement composites (Arora and Dayal 1983 ). The composites were subjected to variable leaching cycles with deionized water. The activity leached as a function of the fractional liquid contact time (the time in each wet-dry cycle during which the waste composite was actually in contact with the influent solution) was compared to that leached for a comparable contact time for a modified International Atomic Ene'gy Agency (IAEA) leach test (Hespe 1971), which represents a continuous saturated type leaching. Their data indicate that the wet-dry cycling results in a pigher release (leaching) of radionuclides than does continuous leaching for the same contact time. The authors suggest that the waste form remains internally saturated and diffusional processes continue largely unabated during the dry periods, and the subsequent wetting of the waste form leaches the radionuclides, which concentrate at the surface during the dry cycle.

The above studies suggest that leaching and transport through unsaturated zones may be appreciable, and possibly significantly greater than that of a comparable saturated zone (on a column volume basis). Therefore, the ability to model leaching and transport of radionuclides in soiis under unsaturated 
conditions is important in assessing the performance of low-level waste disposal sites.

The field experiments described in this report were designed to provide data to develop a capability to predict chemical transport under unsaturated conditions in soils. The experiments were conducted to provide technical support to assist DOE and NRC in improving the understanding of estimated leaching and transport scenarios used to assess the performance of low-level waste disposal sites. The DOE National Low-Level Waste Management Program (NLLWMP) had invested several years investigating the hydraulic properties of the sandy silt backfill used for shallow land burial operations at Los Aidmos and was interested in coupling chemical transport with the water migration studies. Another general purpose of the field experiment was to compare the NRC laboratory-derived parameter estimates with those derived from a joint DOE/NRC large-scale field experiment.

In the following sections, we will first explain the experimental approach and design of our field experiments, describe the materials and methods used in the study, and present only the tracer results pertaining to the DOE portion of the study (iodide, bromide, and lithium). The results will be ciscussed relative to application of several chemical transport models for future modeling. A detailed listing of tracer data is presented in the apperidix for the use of moaelers who will use this data base to field-validate their transport modeis for steady-state and unsteady-state flow conditions in the unsaturated zone.

II. EXPERIMENTAL APPRDACH AND DESIGN

Traditional modeling efforts for radionuclide transport in the unsaturated zone lump the effects of several processes that relate solute 
concentration to concentration in the solid phase in a distribution coefficient, $k_{d}$. This can best be illustrated in tre form of a retardation factor in the one-dimensional convective - dispersive solute transport equation (van Genuchten and Alves 1982) written as

$$
R \frac{\partial c}{\partial t}=D \frac{\partial c}{\partial x^{2}}-v \frac{\partial c}{\partial x}-\mu c+\gamma
$$

where $\mathrm{R}$ is a retardation factor, $\mathrm{c}$ is the solute concentration in $\mathrm{mg} \mathrm{l}^{-1}, \mathrm{t}$ is time in days, $D$ is an apparent dispersion coefficient in $\mathrm{cm}^{2} \mathrm{~d}^{-1}, x$ is distance in $\mathrm{cm}, v$ is the pore velocity in $\mathrm{cm} \mathrm{d}^{-1}, \mu$ is a first-order decay constant in $\mathrm{d}^{-1}$, and $\gamma$ is a zero-order production constant in $\mathrm{mg}^{-1} \mathrm{~d}^{-1}$.

In order to apply Eq. 1 to the modeling of solute transport, the upper or inflow boundary condition needs to be specified. The source term can be used to specify the upper or inflow boundary condition to Eq. 1. For example, a single-pulse input of tracer (representing the concentration of the contaminant leached from waste material) can be represented as

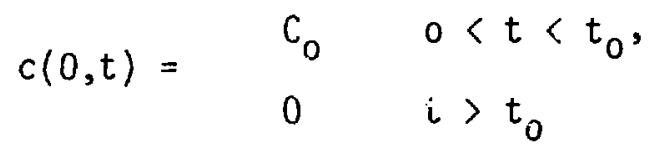

where $t$ is time and $C_{0}$ is input or source term concentration. A square wave input is the sum of several pulse inputs of the form

$$
c_{i}(0, t)=\quad \begin{array}{ll}
c_{0 i} & t_{i-1}<t<t_{i}, \\
0 & t>t_{i}
\end{array}
$$

where $j$ is an index for the individual fulses. If sporadic inputs, such as infiltration following precipitation events are appropriate, then Eq. 3 could be used to approximate the source term. Notice (Eq. 1) that we can have steady-state water movenent ( $\theta$ and $v$ constant), yet have leaching inputs that 
vary in time and in concentration, or we can have unsteady water flow with constant or varying concentration of the leaching input.

The retardation factor, $R$, can be expressed as

$$
R=1+\therefore K_{d} / 0
$$

where $\rho$ is the bulk density in $\mathrm{g} \mathrm{cm}^{-3}, \mathrm{~K}_{\mathrm{d}}$ is the distribution coefficient in $\mathrm{m} \varepsilon \mathrm{g}^{-1}$, and $\theta$ is the volumetric water content. The distribution coefficient $K_{d}$ can, in turn, be expressed as

$$
k_{d}=s / c,
$$

where $s$ is the concentration in the solid phase in ppm or $\mathrm{mg} \mathrm{kg}^{-1}$ and $\mathrm{c}$ is the solute concentration in $\mathrm{mg} \ell^{-1}$. The assumption in Eq. 5 is that the solid and solute concentrations are at equilibrium, which means that flow velocities are low enough so that equilibrium can be obtained.

Other complications are that the distribution coefficient is, in general, not independent of concentration and may vary with moisture content. Moreover, for a particular constituent the value of $k_{d}$ may be influenced by the presence of other dissolved species in sufficiently high concentrations. Other chemical processes that may affect solute transport and, thus, the apparent or effective retardation and $k_{d}$ include precipitation and/or coprecipitation. For additional discussion of these processes and their influence on solute transport, see Arnold et al. 1982, Onishi et al. 1981, Nielsen et al. 1972, and Miller et al. 1965.

Physical processes, such as the relative amount of mobile and immobile water involved in unsaturated flow, can affect solute transport as can 
nonhomogeneous flow processes and fracture flow (e.g., see Amoozegar-Fard et al. 1982 and Wierenga 1977). The dispersion coefficient, D, can be related to micro- and macro-processes through the effective dispersion coefficient as

$$
D=D_{0} \tau+\lambda v,
$$

where $D$ is the effective dispersion coefficient in $\mathrm{cm}^{2} \mathrm{~d}^{-1}, D_{0}$ is the diffusion coefficient in $\mathrm{cm}^{2} \mathrm{~d}^{-1}, \tau$ is a tortuosity factor, $\lambda$ is the dispersivity in $\mathrm{cm}$, and $v$ is the pore velocity in $\mathrm{cm} \mathrm{d}^{-1}$. The dispersivity, $\lambda$, is usually assumed to be a constant but may vary with travel distance. The value of $v$ in Eq. 6 is taken as the mean pore water velocity. In actual systems, $v$ has a distribution about the mean value and dispersivity (and the other parameters) can differ for the longitudinal and the lateral directions (Simmons 1982). Under these conditions, appropriate modeling of solute transport can only be accompi ished with two- or three-dimensional models.

Therefore, contaminant transport in the unsaturated zone is seen to be a conplex process subject to the influence of many factors, including the effects of adsorptive, chemical, and flow processes. To determine parameter values and, thus, to apply the predictive equations (for example Eq. 1), it is necessary to determine the relative effects of adsorptive, chemical, and physical processes and to also determine when, and if, lumped parameters, such as $K_{d}, R, d, \mu$, can be used with confidence.

Another significant problem affecting the applicability of solute transport equations and associated unsaturated flow equations is the extension of laboratory determined parameters to field conditions. It is very difficult or almost impossible to monitor flow and transport processes to determine parameter values under field conditions each time such information is needed 
on flow and transport. Also, field conditions are difficult to replicate in order to derive parameter estimates. On the other hand, it may be that parameters determined under carefully controlled laboratory conditions, while replicated, may not apply under field conditions. Because laboratory methods are quick and inexpensive compared with field studies, there is a definite need for methods to bridge the gap between laboratory methods and in situ determination of unsaturated flow and transport parameters. In our field experiment, we used $3-m$ by $6-m$ caissons at the Los Alamos Experimental Engineered Test Facility (EETF), because experiments performed at this scale are more representative of large-scale field problems than those performed at the scale of small laboratory columns.

In the caisson experiments, we gathered two types of tracer migration data to be used for chemical transport models. In the simplest case, measurements of water and tracer inflow and outflow rates allow mass balance calculations and determination of when steady-state water content and flow conditions are reached. The other type of data is derived from soil solution samples from the tuff in the caisson at various depths to determine tracer movement and dispersion rates as functions of time and depth. Volumetric water content was also determined as a function of horizontal distance from the edge of the caisson using neutron moisture gauge techniques at six sampling ports located at incremental depth intervals of $75 \mathrm{~cm}$. The sampling ports also allow for soil samples to be collected at the end of the experiment as a function of depth to be assayed for tracers, which are tightly adsorbed to the backfill matrix and exhibit minimal mobility. Although the removal of samples from the sampling ports with this approach will disturb steady-state conditions, the perturbations will be minimal because of the scale of the caisson experiments and should not affect the conditions of the experiment. 
In the experimental design of our caisson studies, nonsorbing tracers (iodide and bromide) were used to determine dispersion coefficients for solute transport through soil, and sorbing tracers (cesium, strontium, and lithium) wera used to determine retardation factors for a range of ion adsorption affinities. Flow conditions included a 6 -day tracer pulse followed by a steady-state flow rate of about $4 \mathrm{~cm} \mathrm{~d}^{-1}\left(200 \mathrm{~cm}^{3} \mathrm{~min}^{-1}\right)$ and a 6 -day tracer pulse followed by unsteady-state flow; two steady-state and two unsteddy-state pulses were performed in FY85. In the subsequent 70-day period following the single tracer input when only calcium chloride solution was added (no tracer), soil solution samples were collected daily at incremental depths and analyzed for tracer concentrations. In addition, inflow and outflow samples were collected and analyzed for tracer concentrations and outflow rates were measured. Neutron moisture data were also collected biweekli; as functions of depth and horizortal distance within the caisson.

Since one of the purposes of our caisson experiment is to ultimately compare the NRC laboratory-derived parameter estimates with those derived from the caisson, this exferimental design will provide unique data for evaluating the applicability of lumped coefficients in modeling solute transport under controlled conditions in the laboratory as well as il the large caissons. Thus, in our first pulse experiment, the caisson received a single-pulse input of bromide and iodide (conservative and nonadsorbing tracers) as well as lithium, strontium, and cesium under unsteady-state flow conditions. The bromide and iodide tracer data were used to determine values of $D_{0}, \tau$, and $\gamma$, where $D_{0}$ is the diffusion coefficient in $\mathrm{cm}^{2} \mathrm{~d}^{-1}, \tau$ is a tortuosity factor, and $\gamma$ is the dispersivity in $\mathrm{cm}$. These values were used to estimate effective dispersion coerficients for subsequent analys is of the retardation factor ( $R$ ) 
and the distribution coefficient $\left(K_{d}\right)$ for lithium and strontium using least-squares procedures (van Geruchten and ATves 1982).

This experimentai design allowed us to gather information on a field-scale study to address two other issues: replication of paraneter estimates and application of parameter estimates in predicting steady-state and unsteady-state solute transport. The results from these series of pulse inputs will allow better estimates of the sampling variability of $D_{0}, \tau$, and $\gamma$, thus, testing how well replicated tracer inputs result in the reproduction of data-based parameter estimates. In addition, because two types of flow conditions were studied, experimental data could address how rapidly (with respect to depth and time) does the unsteady response to a pulse input approach steady-state conditions. If the unsteady response to a pulse rapidly approaches steady-state conditions, then steady-state models may be applicable to predicting transport in unsteady conditions. The complexities of applying unsteady-state models is horrendous. In addition, do we obtain similar data-based parameter estimates under steady-state and unsteady flow conditions? Again, the issue has practical importance in determining when the application of a steady-state model is appropriate and in interpretation of parameter estimates from unsteady flow and transport data.

\section{II . MATERIALS AND METHODS}

The caisson (Caisson B) used in this field experiment is located in an experiment cluster (DePoorter 1981) at the EETF (the development of this site was sponsored by the DOE NLLWMP). The experiment cluster consists of six corrugated metal pipes ( $3 \mathrm{~m}$ in diameter and $6 \mathrm{~m}$ deep) placed around a central instrument and access caisson of the same size (Fig. 2). In five of the six interstitial positions, there are pipes $46 \mathrm{~cm}$ in diameter and $6 \mathrm{~m}$ 1ong. 
Access ports are situated at $75-\mathrm{cm}$ depth interyals between the central caisson and each of the eleven surrounding caissons (the access ports are shown in detail in Fig. 3) and allow the study of chemical transport as a function of depth in the caisson. The inside of each caisson was coated with Teflon paint in an effort to minimize tracer adsorption on the surface of the caisson walls. Caisson B was filled with crushed Bandelier Tuff, which consists mostly of silicic glass and has a grain-size distribution similar to that of a silty sand. At the bottom of the caisson, the drain was covered with a coarse screen. Approximately $0.25 \mathrm{~m}$ of gravel (approximately 2- to 3-cm diameter) was placed over the screen, and above this was placed approximately $0.25 \mathrm{~m}$ of coarse sand. The rest of the caisson was filled with crushed Bandelier Tuff, which was obtained locally.

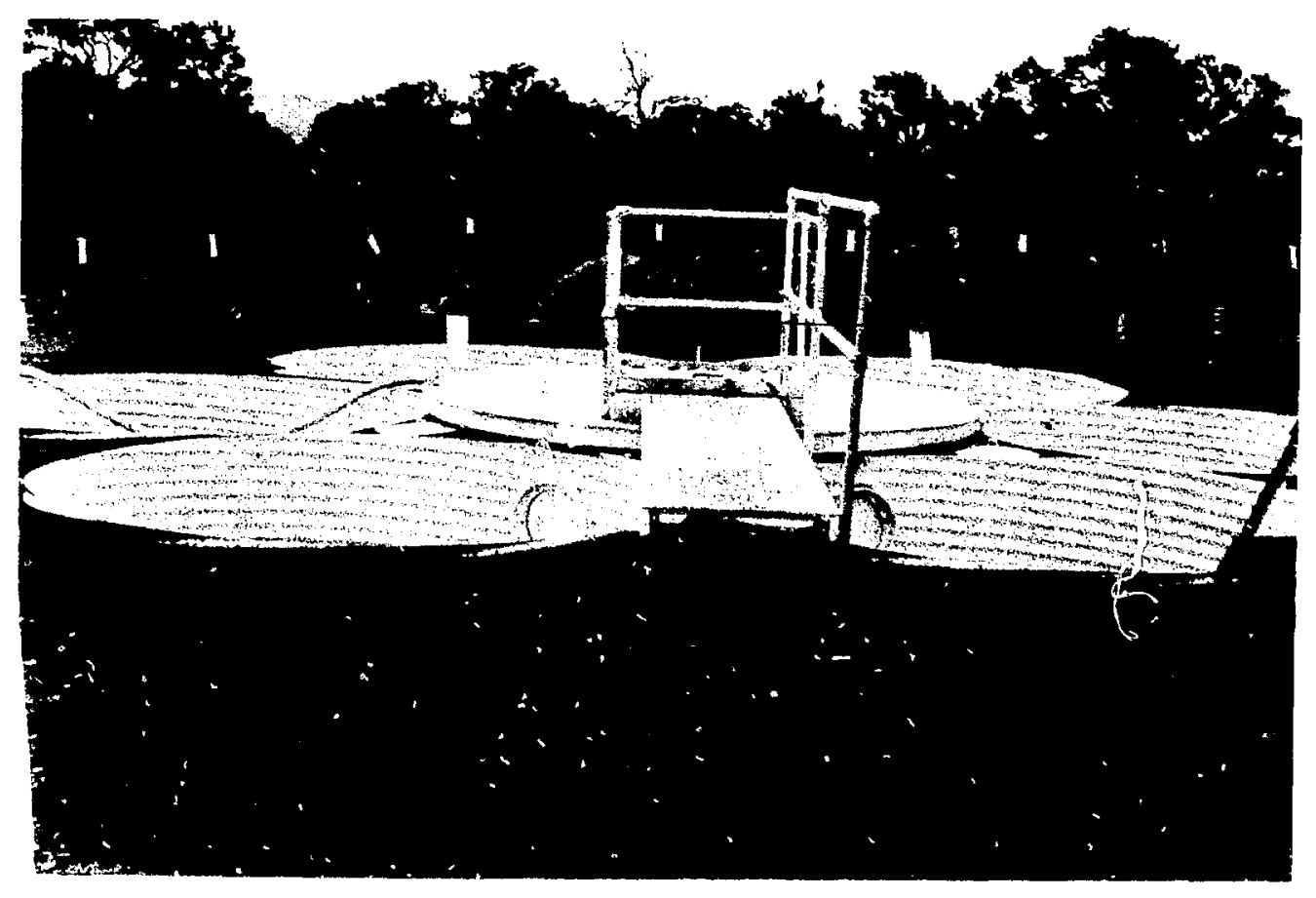

Fig. 2. Experimental cluster at the EETF. 
After crushing, the tuff was screened at a cement batch plant. All Inaterial passing through a 0.0125 in screen was mixed with known amounts of water to give optinum water content for compaction ensuring column uniformity and minimal subsidence. Water content as well as wet and dry densities were determined at several caisson depths during the filling operation by a Portaprobe Model MC-2 Percent-Test Nuclear Meter. The tuff was compacted by use of tampers (Mikasa Model MTR-80G) after each 0.2-m-thick layer was added. Dry densities varied with depth from 92.8 (at the $1.56-\mathrm{m}$ depth) to $99.3 \%$ (at the $0.25-\mathrm{m}$ depth) of what is considered optimum dry density for crushed tuff. Thus, maximum compaction was not obtained at the $1.56-\mathrm{m}$ depth where the initial moisture content was too high for optimal compaction. Average void ratios, which were calculated from the dry density data, were $0.60,0.67$, $0.71,0.67,0.62,0.64$, and 0.64 for caisson depths of $0.25,1.14,1.56,2.72$, $3.59,4.14$, and $4.92 \mathrm{~m}$, respectively.

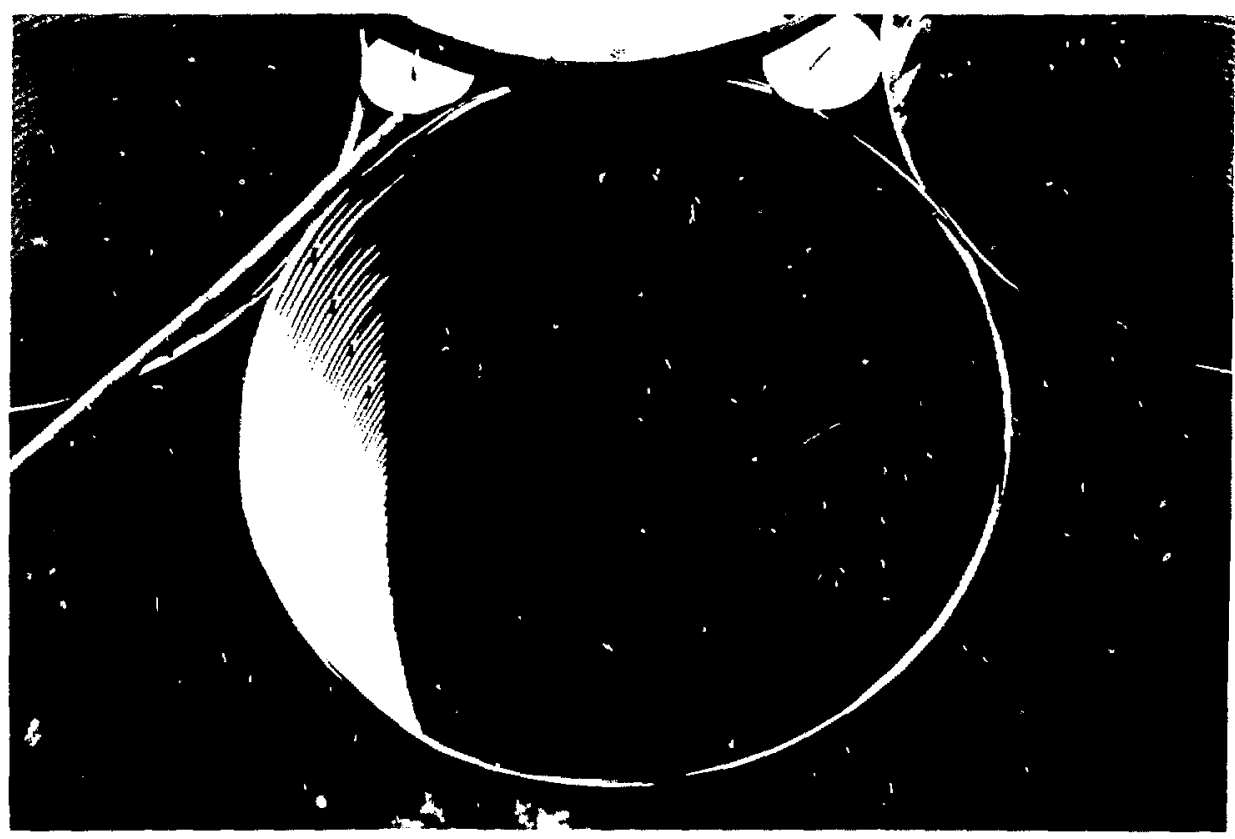

Fig. 3. Access port details in empty caisson. 
The geohydrology of crushed Bandelier Tuff has been extensively characterized (Abeele et al. 1981). Conductivities ranging from $9.2 \times 10^{-7} \mathrm{~m}$ $\mathrm{s}^{-1}$ at saturation to $9.9 \times 10^{-11} \mathrm{~m} \mathrm{~s}^{-1}$ at $25 \%$ of saturation have been measured (Abeele et al. 1981).

One of the disadvantages of performing field research under unsaturated flow conditions at the scale used in our caisson experiments was that the technology needed to do the experiment did not exist. Thus, we had to develop (1) an effective water-solute distribution systen at the surface of the caisson, (2) a system for blending and storing the calcium chloride and tracer solutions, and (3) nonreactive devices to sample groundwater. Over two years' worth of efforts on those subjects are summarized in detail by cokal et al. 1985.

The water-solute distribution systeri makes use of an assembly of four fluidic wafer switches (trade name, SCANIVALVES), each of which is fitted with 24 outlet ports fed sequentially from a single peristaltic pump, which is fitted with an electronic flow metering system (SIGNET Micro Flo-Sensor). The pump speed is adjusted as required to provide the necessary flow rate (about $\left.200 \mathrm{~cm}^{3} \mathrm{~min}^{-1}\right)$. The four ganged 24-position wafer switches are capable of delivering solution to 96 distinct points upon the surface of the caisson, with four randomly chosen points simultaneously receiving solution flow at any. given instant. The SCANIVALVES are advanced sequentially by a stepping motor drive, which, in turn, receives its power impulse from a battery-powered relaxation oscillator. The oscillator is set to deliver impulses at 12-second intervals. Thus, the entire set of outlets receives solution every 288 seconds, or approximately every five minutes.

Tests of delivery uniformity were conducted over a $21 / 2$ month period between June and September 1984. In these tests, solution was collected from 
each of the 96 outlets for one delivery period, each working day. The delivered volume was found to have day-to-day reproducibility (1 sigma probability level) within $5 \%$ of the mean delivered volume for the particular head tested.

A continuous application of $0.005 \mathrm{~F}$ calcium chloride solution (200 mg $\hat{i}^{-1}$ as $\mathrm{Ca}$ ) containing target concentrations of tracers of $0.001 \mathrm{M}$, was required for our experiments. It was necessary to provide for continuous blending and storage of this solution as a separate unit operation. Thus, the assembled blending/storage system (Fig. 4) contains the follo ing components:

1. Water storage tank, 5000 \& capacity (1200 gallons), with flow meter and flow adjustment valve.

2. Concentrate storage tank, $200 \ell$.

3. Blended matrix solution storage tank, $1600 \ell$, with filter at outflow points.

4. Concentrate metering pumps (two) and blending chamber.

5. Interval sampler, timer controlled, for collection of a composite sample of the matrix solution delivered to the caisson distribution system.

The concentrated tracer solutions were made daily by adding $543 \mathrm{~g} \mathrm{KI}$, $217 \mathrm{~g} \mathrm{LiBr}$, and $1384 \mathrm{~g} \mathrm{CaCl}_{2}$ to 25 \& of distilled water. After this concentrated solution was thoroughly mixed, it was diluted in the concentrate storage tank at a $1: 100$ ratio.

The materials of construction of the system were selected to preclude contamination of the matrix solution by corrosive products. The water and concentrate storage tanks are made of polyethylene, the matrix storage tank is made of an epoxy/fiberglass composite, and all plumbing fittings are either Teflon, PVC, or stainless steel and fittings are sealed with Teflon sealing 


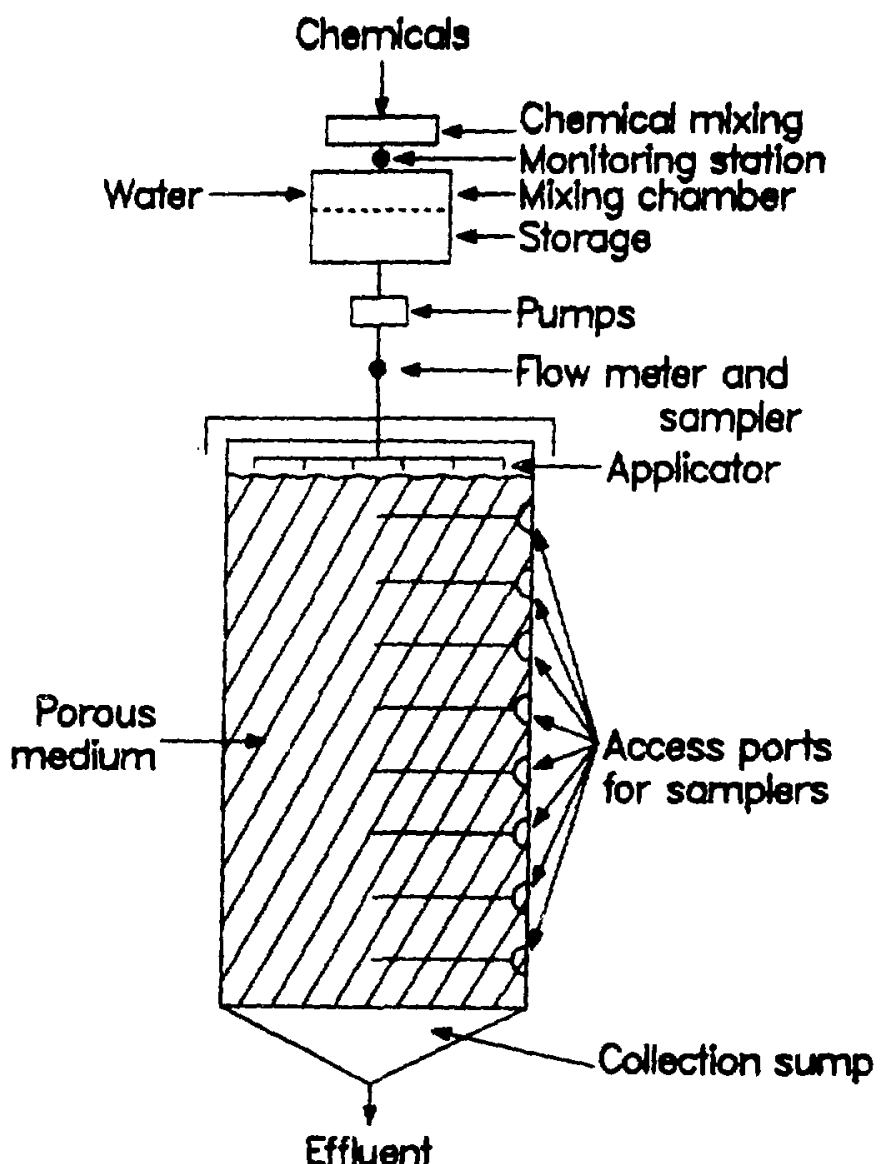

Fig. 4. Field caisson configuration.

tape. Since no brass or iron fittings are used, no corrodible materials contact the solution at any point. This entire system is insulated and equipped with heating tape to allow experiments to be performed throughout the subzero conditions experienced during the winter months.

The initial and current operations of this system did not fully implement the blending feedbaik control system. At present, the blending is operated bv a 24-hour timer switch instead of the ion electrode and meter system initially 
installed. The timer allows operations of the concentrate feed pump and a solenoid valve in the water feed iine. The blended matrix solution is sampled manually at the point of introduction to the matrix storage tank. It has been found that this rudimentary control is actually sufficient for the present purpose because minor concentration fluctuations in the blended solution are attenuated by the presence of about 1000-1600\& of matrix solution in the storage tank. The inflow is sampled on a daily basis while the blending system is in operation, anc the calcium analysis result is used to adjust the water or concentrate feed rates as necessary to maintain the desired calcium concentration in the matrix solution. The composite sample representing the actual matrix solution delivered to the caisson distribution system is al so analyzed to provide information on the constancy of the delivered eluent.

During our tracer transport studies, it was also necessary to ohtain a representative saficle of the soil solution as a function of time and sampling depth. Ceramic cup samplers normally used by soil scientists and groundwater nydrologists were found to substantially adsorb cationic tracers (Cokal et al. 1985) and, thus, were replaced with Teflon porous cups. The Teflon porous cups were satisfactory from the adsorption staridpoint, however, horizontal emplacement of these devices in the raissor, system proved to be unexpectedly difficult. The difficulty arose from the fact that porous cup samplers function by maintaining a continuous moisture film between the porous cup and the surrounding soil (or tuff) backfill material. Teflon, being hydrophobic, and probably depending for its non-adsorptive properties upon this fact, does not readily support the continuous moisture $f i l m$ between the porous cup and backfill material, making collection of soil solution samples below saturation impossible. Further consideration of this point led to the conclusion that the porous Teflon cups (when emplaced according to the manufacturer's 
recommended procedure) were actually functioning as inert supports for the silica flour backfill material. The silica flour was probably the actual porous matrix through which the sample was collected.

The problems encountered in reliably deploying the Teflon porous cup samplers led to a reexamination of the sampler installations. Because previous workers (Jackson et al. 1976) had reported on the successful use of porous hollow fiber bundles in soil column sampling work, a procedure was developed to prepare and emplace similar hollow fiber bundles in the backfill in the caissons. Hydrophilic fluoropolymer fibers of $20-\mathrm{cm}$ length, with a porosity equivalent to about 100,000 molecular weight, were bundled and then cemented into a Nylon SWAGELOK fitting. The assembled fiber bundle was finally fitted with a suitable length of $1 / 4$ " polyethylene tubing and emplaced in the caisson borehole within a plug of slurried, -100 mesh tuff or tuff/ silica flour mixture. The required water samples were then collected by drawing a suitable vacuum (about $22 \mathrm{~cm}$ ) on the system. This system included a polyethylene centrifuge tube used for receiving the soil solution sample and for transport of the sample to the laboratory for analysis.

Samples of soil solution were collected using the hollow fiber samplers at several sampling depths in the caisson. One set of samples was collected at each of six access ports (at depths of $43,119,194,271,347$, and $421 \mathrm{~cm}^{*}$ ) with the idea of characterizing the relatively fast transport of the nonsorbing tracers such as iodide and bromide. A second set of samples was collected starting in February 1985 using a series of vertically-emplaced samplers located at depths of $43,58,73,88,103$, and $118 \mathrm{~cm}$. The latter set of samplers was to be used (1) to detect the migration of the slow-moving absorbing tracers (cesium and strontium), and (2) to determine differences in

*Corresponds to soit sampling depths of $36,113,188,264,339$, and $415 \mathrm{~cm}$ respectively. 
modeling chemical transport of tracers using a data set from a smaller depth increment system than the first set of samples which were collected throughout the entire depth of the caisson.

Several techniques were used to assay the soil solution samples for iadide, bromide, and lithium. Iodide assays were performed with an ion selective electrode. Bromide assays were usually performed using ion chromatography, except for the samples from the second unsteady-state pulse, which were assayed using neutron activation techniques. Lithium assays were performed by $\mathrm{flame}$ atomic adsorption spectrophotometry procedures.

Volumetric water content was determined using a Campbell Pacific Neutron Moisture Gauge (Model 5030R). Measurements were made within al uminum access tubes located at each of the six access ports throughout the caisson at distances of 0 to $130 \mathrm{~cm}$ from the caisson wall in $10-\mathrm{cm}$ increments. Volumetric water content was also determined in a vertical access tube for depths ranging from 20 to $560 \mathrm{~cm}$ in increments of $20 \mathrm{~cm}$.

\section{RESULTS AND DISCUSSION}

Although the major emphasis is placed on presenting the tracer data in the succeeding sections, a tremendous amount of soil water data was also collected (see Appendix A). Volumetric water content at all sampling depths (43 to $421 \mathrm{~cm}$ ) remained essentially constant throughout the first unsteady-state pulse experiment and the two following unsteady-state pulse experiments at values that normally ranged from 27 to $29 \%$. Because our crushed tuff had a volumetric water content of $33 \%$ at saturation, this means that these pulse experiments were performed at 82 to $88 \%$ of saturation. The second unsteady-state pulse started out and remained in this moisture regime for 21 days, at which time no solution was added to the caisson; eleven days 
after the solution flow was terminated, the tuff throughout the caisson was at approximately $50 \%$ saturation.

In addition to the volumetric water content data collected during these experiments, some soil moisture tension data were also collected in 1985 using well-established methods (Abeele 1984). Tension data are presented in Tables 1 and 2 for measurements collected at the $43-\mathrm{cm}$ and $347-\mathrm{cm}$ depths, respectively. Soil water tension values at both depths in the caisson generally ranged from 2 to 8 inches of water during the steady-state flow experiments, except for the drainage portion of the second unsteady-state pulse experiment between April 29 and July 11, 1985. During this time, the tension increased to values close to 30 inches of water at the $43-\mathrm{cm}$ depth (Table 1) and about 18 inches of water at the 347-cm depth (Table 2), as would be expected.

The outflow data collected in Caisson B are presented in Table 3 for measurements made between July 1984 and October 1985. (Note that tracer concentrations in these samples are also presented in Appendix B.) Outflow rates ranging from 110 to $250 \mathrm{~cm}^{3} \mathrm{~min}^{-1}$ were observed during most of the steady-state flow experiments, values closer to our target flow rate of 200 $\mathrm{cm}^{3} \min ^{-1}$. During the portion of the second unsteady-state pulse when no solution was added to the caisson, out low rates dropped to a minimum of $3 \mathrm{~cm}^{3}$ $\min ^{-1}$ (Table 3).

Because our field experiments were conducted throughout the winter of 1984 and the summers of 1984 and 1985, we felt that soil temperatures inside and outside of the caisson should be monitored. Thus, temperature profiles were determined (DePoorter 1981) at various depths (Table 4) and horizontal locations (Table 5) within the caisson, and compared with similar temperature measurements collected outside of the caisson (Table 6). The data collected 
show that our winterizing efforts resulted in soil temperatures in the caisson of only a few degrees in the months of January and February 1985, with temperatures of 18 to $20^{\circ} \mathrm{C}$ being more common in the summer of 1985 (Tables 4 and 5). Horizontal temperature gradients were generally minimal (Table 5), and temperature profiles outside of the caisson varied seisonally with similar vertical profiles collected within the caisson (compare iables 4 and 6 ).

In the following sections, the tracer data from Caisson $B$ are discussed in terms of the data collected from the samplers located at the caisson access ports and the vertical samplers located in the upper $118 \mathrm{~cm}$ of th caisson. This data base is presented in deiail in Appendix B for future use by chemical transport modelers. In addition, our preliminary parameter estimates for the tracer dispersion coefficients and retardation factors are presented.

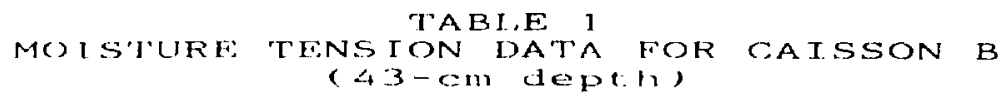

\begin{tabular}{|c|c|c|c|c|c|}
\hline Date & $\begin{array}{l}\text { Tension } \\
\left(\mathrm{In}-\mathrm{H}_{2} \mathrm{O}\right)\end{array}$ & Date & $\begin{array}{l}\text { Tension } \\
\left(\mathrm{In}-\mathrm{H}_{2} \mathrm{O}\right)\end{array}$ & Date & $\begin{array}{l}\text { Tension } \\
\left(\mathrm{In}-\mathrm{H}_{2} \mathrm{O}\right)\end{array}$ \\
\hline $\begin{array}{l}3 / 30 / 85 \\
3 / 31 / 85 \\
4 / 2 / 85 \\
4 / 3 / 85 \\
4 / 5 / 85\end{array}$ & $\begin{array}{l}2.0 \\
4.0 \\
4.0 \\
4.0 \\
6.0\end{array}$ & $\begin{array}{l}6 / 11 / 85 \\
6 / 17 / 85 \\
6 / 19 / 85 \\
6 / 20 / 85 \\
6 / 21 / 85\end{array}$ & $\begin{array}{l}26.0 \\
28.0 \\
30.0 \\
29.5 \\
29.0\end{array}$ & $\begin{array}{l}7 / 29 / 85 \\
7 / 31 / 85 \\
8 / 2 / 85 \\
8 / 7 / 85 \\
8 / 9 / 85\end{array}$ & $\begin{array}{l}4.0 \\
4.0 \\
4.0 \\
4.0 \\
4.0\end{array}$ \\
\hline $\begin{array}{l}4 / 8 / 85 \\
4 / 10 / 85 \\
4 / 12 / 85 \\
4 / 14 / 85 \\
4 / 16 / 85\end{array}$ & $\begin{array}{l}5.5 \\
6.0 \\
6.0 \\
5.5 \\
5.0\end{array}$ & $\begin{array}{l}6 / 22 / 85 \\
6 / 23 / 85 \\
6 / 24 / 85 \\
6 / 25 / 85 \\
6 / 27 / 85\end{array}$ & $\begin{array}{l}31.0 \\
31.0 \\
30.0 \\
31.0 \\
30.0\end{array}$ & $\begin{array}{l}8 / 14 / 85 \\
8 / 16 / 85 \\
8 / 13 / 85 \\
8 / 23 / 85 \\
8 / 26 / 85\end{array}$ & $\begin{array}{l}4.0 \\
4.5 \\
4.0 \\
4.0 \\
4.0\end{array}$ \\
\hline $\begin{array}{l}\downarrow / 18 / 85 \\
4 / 20 / 85 \\
4 / 22 / 85 \\
4 / 24 / 85 \\
4 / 25 / 85\end{array}$ & $\begin{array}{l}4.0 \\
4.5 \\
4.0 \\
4.0 \\
4.5\end{array}$ & $\begin{array}{l}6 / 29 / 85 \\
6 / 30 / 85 \\
7 / 2 / 85 \\
7 / 4 / 85 \\
7 / 5 / 85\end{array}$ & $\begin{array}{l}30.0 \\
29.0 \\
30.0 \\
32.0 \\
29.5\end{array}$ & $\begin{array}{l}8 / 28 / 85 \\
8 / 30 / 85 \\
9 / 3 / 85 \\
9 / 5 / 85 \\
9 / 16 / 85\end{array}$ & $\begin{array}{l}4.0 \\
4.0 \\
4.0 \\
4.0 \\
3.0\end{array}$ \\
\hline $\begin{array}{l}4 / 26 / 85 \\
4 / 28 / 85 \\
4 / 29 / 85 \\
4 / 30 / 85 \\
5 / 1 / 85\end{array}$ & $\begin{array}{l}4.0 \\
3.0 \\
4.0 \\
6.0 \\
8.0\end{array}$ & $\begin{array}{l}7 / 6 / 85 \\
7 / 7 / 85 \\
7 / 9 / 85 \\
7 / 17 / 85 \\
7 / 13 / 85\end{array}$ & $\begin{array}{l}29.0 \\
29.0 \\
30.0 \\
30.0 \\
10.0\end{array}$ & $\begin{array}{r}9 / 18 / 85 \\
9 / 20 / 85 \\
9 / 25 / 85 \\
10 / 2 / 85 \\
10 / 9 / 85\end{array}$ & $\begin{array}{l}4.0 \\
3.0 \\
2.0 \\
2.0 \\
2.0\end{array}$ \\
\hline $\begin{array}{l}5 / 2 / 85 \\
5 / 6 / 85 \\
5 / 8 / 85 \\
5 / 10 / 85 \\
5 / 13 / 85\end{array}$ & $\begin{array}{l}10.0 \\
12.0 \\
13.0 \\
12.0 \\
15.0\end{array}$ & $\begin{array}{l}7 / 14 / 85 \\
7 / 15 / 85 \\
7 / 16 / 85 \\
7 / 17 / 85 \\
7 / 18 / 85\end{array}$ & $\begin{array}{l}8.0 \\
6.0 \\
4.0 \\
5.0 \\
5.5\end{array}$ & & \\
\hline $\begin{array}{l}5 / 15 / 85 \\
5 / 23 / 85 \\
5 / 28 / 85 \\
5 / 30 / 85 \\
6 / 4 / 85\end{array}$ & $\begin{array}{l}18.0 \\
20.0 \\
20.0 \\
22.0 \\
24.0\end{array}$ & $\begin{array}{l}7 / 19 / 85 \\
7 / 21 / 85 \\
7 / 23 / 85 \\
7 / 25 / 85 \\
7 / 27 / 85\end{array}$ & $\begin{array}{l}4.0 \\
4.5 \\
5.0 \\
4.0 \\
4.5\end{array}$ & & \\
\hline
\end{tabular}


TABLE 2

MOISTURE TENSION DATA FOR CAISSON B (347-cm depth)

\begin{tabular}{|c|c|c|c|c|c|}
\hline Date & $\begin{array}{l}\text { Tension } \\
\left(\text { in }-\mathrm{H}_{2} \mathrm{O}\right)\end{array}$ & Date & $\begin{array}{l}\text { Tension } \\
\left(\text { in }-\mathrm{H}_{2} \mathrm{O}\right)\end{array}$ & Date & $\begin{array}{l}\text { Tension } \\
\left(\text { in- } \mathrm{H}_{2} \mathrm{O}\right)\end{array}$ \\
\hline $\begin{array}{l}3 / 30 / 85 \\
3 / 31 / 85 \\
4 / 2 / 85 \\
4 / 3 / 85 \\
4 / 5 / 85\end{array}$ & $\begin{array}{r}2.5 \\
18.0 \\
4.0 \\
3.0 \\
3.0\end{array}$ & $\begin{array}{l}6 / 11 / 85 \\
6 / 17 / 85 \\
6 / 19 / 85 \\
6 / 20 / 85 \\
6 / 21 / 85\end{array}$ & $\begin{array}{l}18.0 \\
18.0 \\
19.0 \\
18.5 \\
19.0\end{array}$ & $\begin{array}{l}7 / 29 / 85 \\
7 / 31 / 85 \\
8 / 2 / 85 \\
8 / 7 / 85 \\
8 / 9 / 85\end{array}$ & $\begin{array}{l}3.0 \\
3.0 \\
3.0 \\
3.5 \\
3.0\end{array}$ \\
\hline $\begin{array}{l}4 / 8 / 85 \\
4 / 10 / 85 \\
4 / 12 / 85 \\
4 / 14 / 85 \\
4 / 16 / 85\end{array}$ & $\begin{array}{l}3.0 \\
2.5 \\
3.0 \\
2.5 \\
3.5\end{array}$ & $\begin{array}{l}6 / 22 / 85 \\
6 / 23 / 85 \\
6 / 24 / 85 \\
6 / 25 / 85 \\
6 / 27 / 85\end{array}$ & $\begin{array}{l}19.0 \\
12.5 \\
13.0 \\
14.0 \\
14.0\end{array}$ & $\begin{array}{l}8 / 14 / 85 \\
8 / 16 / 85 \\
8 / 19 / 85 \\
8 / 23 / 85 \\
8 / 26 / 85\end{array}$ & $\begin{array}{l}3.0 \\
3.0 \\
3.0 \\
3.0 \\
3.0\end{array}$ \\
\hline $\begin{array}{l}4 / 18 / 85 \\
4 / 20 / 85 \\
4 / 22 / 85 \\
4 / 24 / 85 \\
4 / 25 / 85\end{array}$ & $\begin{array}{l}3.0 \\
2.5 \\
3.0 \\
3.0 \\
3.5\end{array}$ & $\begin{array}{l}6 / 29 / 85 \\
6 / 30 / 85 \\
7 / 2 / 85 \\
7 / 4 / 85 \\
7 / 5 / 85\end{array}$ & $\begin{array}{l}14.0 \\
14.0 \\
13.5 \\
15.0 \\
18.0\end{array}$ & $\begin{array}{l}8 / 28 / 85 \\
8 / 30 / 85 \\
9 / 3 / 85 \\
9 / 5 / 85 \\
9 / 16 / 85\end{array}$ & $\begin{array}{l}3.0 \\
3.0 \\
3.0 \\
3.0 \\
3.0\end{array}$ \\
\hline $\begin{array}{l}4 / 26 / 85 \\
4 / 28 / 85 \\
4 / 29 / 85 \\
4 / 30 / 85 \\
5 / 1 / 85\end{array}$ & $\begin{array}{l}3.0 \\
3.0 \\
3.0 \\
4.0 \\
4.0\end{array}$ & $\begin{array}{l}7 / 6 / 85 \\
7 / 7 / 85 \\
7 / 9 / 85 \\
7 / 11 / 85 \\
7 / 13 / 85\end{array}$ & $\begin{array}{l}19.0 \\
18.0 \\
17.0 \\
16.5 \\
17.0\end{array}$ & $\begin{array}{r}9 / 18 / 85 \\
9 / 20 / 85 \\
9 / 25 / 85 \\
10 / 2 / 85 \\
10 / 9 / 85\end{array}$ & $\begin{array}{l}3.0 \\
3.0 \\
3.0 \\
3.0 \\
3.0\end{array}$ \\
\hline $\begin{array}{l}5 / 2 / 85 \\
5 / 6 / 85 \\
5 / 8 / 85 \\
5 / 10 / 85 \\
5 / 13 / 85\end{array}$ & $\begin{array}{r}4.0 \\
5.0 \\
7.0 \\
8.0 \\
10.0\end{array}$ & $\begin{array}{l}7 / 24 / 85 \\
7 / 25 / 85 \\
7 / 16 / 85 \\
7 / 17 / 85 \\
7 / 18 / 85\end{array}$ & $\begin{array}{l}17.0 \\
17.0 \\
17.0 \\
17.0 \\
17.5\end{array}$ & & \\
\hline $\begin{array}{l}5 / 15 / 85 \\
5 / 23 / 85 \\
5 / 28 / 85 \\
5 / 30 / 85 \\
6 / 4 / 85\end{array}$ & $\begin{array}{l}10.0 \\
14.0 \\
13.0 \\
15.8 \\
16.0\end{array}$ & $\begin{array}{l}7 / 19 / 85 \\
7 / 21 / 85 \\
7 / 23 / 85 \\
7 / 25 / 85 \\
7 / 27 / 85\end{array}$ & $\begin{array}{r}17.0 \\
6.5 \\
4.0 \\
3.0 \\
3.5\end{array}$ & & \\
\hline
\end{tabular}


TABLE 3

CAISSON B OUTFLOW RATE (1984-1985)

\begin{tabular}{|c|c|c|c|c|c|}
\hline Date & $\begin{array}{l}\text { Days } \\
\text { elapsed }\end{array}$ & $\begin{array}{l}\text { outelow } \\
\text { (ml/min) }\end{array}$ & Date & $\begin{array}{c}\text { Days } \\
\text { elapsed }\end{array}$ & $\begin{array}{l}\text { outflow } \\
\text { (ml/min) }\end{array}$ \\
\hline $\begin{array}{ll}7 / 1 / 84 & 1 / 8 \\
8 / & 1 \\
8 / & 2 \\
8 / & 3 \\
8 / & 4\end{array}$ & $\begin{array}{l}-81 \\
-50 \\
-49 \\
-48 \\
-47\end{array}$ & $\begin{array}{l}175 \\
205 \\
210 \\
210 \\
198\end{array}$ & $\begin{array}{l}8 / 25 \\
8 / 26 \\
8 / 27 \\
8 / 29 \\
8 / 30\end{array}$ & $\begin{array}{l}-26 \\
-25 \\
-24 \\
-22 \\
-21\end{array}$ & $\begin{array}{l}191 \\
182 \\
176 \\
174 \\
170\end{array}$ \\
\hline $\begin{array}{ll}8 / & 5 \\
8 / & 6 \\
8 / & 7 \\
8 / & 8 \\
8 / & 9\end{array}$ & $\begin{array}{l}-46 \\
-45 \\
-44 \\
-43 \\
-42\end{array}$ & $\begin{array}{l}174 \\
168 \\
174 \\
197 \\
219\end{array}$ & $\begin{array}{ll}8 / 31 \\
9 / & 1 \\
9 / & 2 \\
9 / & 3 \\
9 / & 4\end{array}$ & $\begin{array}{l}-20 \\
-19 \\
-18 \\
-17 \\
-16\end{array}$ & $\begin{array}{l}177 \\
176 \\
230 \\
210 \\
168\end{array}$ \\
\hline $\begin{array}{l}8 / 10 \\
8 / 11 \\
8 / 12 \\
8 / 13 \\
8 / 14\end{array}$ & $\begin{array}{l}-41 \\
-40 \\
-39 \\
-38 \\
-37\end{array}$ & $\begin{array}{l}220 \\
192 \\
175 \\
170 \\
169\end{array}$ & $\begin{array}{l}9 / 5 \\
9 / 6 \\
9 / 7 \\
9 / 11 \\
9 / 12\end{array}$ & $\begin{array}{l}-15 \\
-14 \\
-13 \\
-9 \\
-8\end{array}$ & $\begin{array}{l}160 \\
146 \\
145 \\
156 \\
155\end{array}$ \\
\hline $\begin{array}{l}8 / 15 \\
8 / 16 \\
8 / 17 \\
8 / 18 \\
8 / 19\end{array}$ & $\begin{array}{l}-36 \\
-35 \\
-34 \\
-33 \\
-32\end{array}$ & $\begin{array}{l}163 \\
165 \\
174 \\
178 \\
176\end{array}$ & $\begin{array}{l}9 / 13 \\
9 / 14 \\
9 / 17 \\
9 / 19 \\
9 / 20\end{array}$ & $\begin{array}{l}-7 \\
-6 \\
-3 \\
-1 \\
0\end{array}$ & $\begin{array}{l}152 \\
146 \\
172 \\
172 \\
171\end{array}$ \\
\hline $\begin{array}{l}8 / 20 \\
8 / 21 \\
8 / 22 \\
8 / 23 \\
8 / 24\end{array}$ & $\begin{array}{l}-31 \\
-30 \\
-29 \\
-28 \\
-27\end{array}$ & $\begin{array}{l}172 \\
172 \\
182 \\
202 \\
200\end{array}$ & $\begin{array}{l}9 / 21 \\
9 / 24 \\
9 / 25 \\
9 / 26 \\
9 / 27\end{array}$ & $\begin{array}{l}1 \\
4 \\
5 \\
6 \\
7\end{array}$ & $\begin{array}{l}173 \\
140 \\
135 \\
126 \\
110\end{array}$ \\
\hline
\end{tabular}


Caisson-B Outflow Rate (Cont.)

\begin{tabular}{|c|c|c|c|c|c|}
\hline Date & $\begin{array}{l}\text { Days } \\
\text { elapsed }\end{array}$ & $\begin{array}{l}\text { outflow } \\
\text { (ml/min) }\end{array}$ & Date & $\begin{array}{l}\text { Days } \\
\text { elapsed }\end{array}$ & $\begin{array}{l}\text { Outflow } \\
(\mathrm{ml} / \mathrm{min})\end{array}$ \\
\hline $\begin{array}{l}9 / 28 \\
10 / 3 \\
10 / 2 \\
10 / 3 \\
10 / 4\end{array}$ & $\begin{array}{r}8 \\
11 \\
12 \\
13 \\
14\end{array}$ & $\begin{array}{r}100 \\
80 \\
70 \\
70 \\
70\end{array}$ & $\begin{array}{l}11 / 8 \\
11 / 12 \\
11 / 14 \\
11 / 15 \\
11 / 19\end{array}$ & $\begin{array}{l}49 \\
53 \\
55 \\
56 \\
60\end{array}$ & $\begin{array}{l}152 \\
170 \\
172 \\
170 \\
184\end{array}$ \\
\hline $\begin{array}{l}10 / 5 \\
10 / 9 \\
10 / 10 \\
10 / 11 \\
10 / 12\end{array}$ & $\begin{array}{l}15 \\
19 \\
20 \\
21 \\
22\end{array}$ & $\begin{array}{r}70 \\
135 \\
118 \\
100 \\
92\end{array}$ & $\begin{array}{l}11 / 21 \\
11 / 23 \\
11 / 26 \\
11 / 28 \\
11 / 29\end{array}$ & $\begin{array}{l}62 \\
64 \\
67 \\
69 \\
70\end{array}$ & $\begin{array}{l}176 \\
166 \\
192 \\
196 \\
194\end{array}$ \\
\hline $\begin{array}{l}10 / 15 \\
10 / 17 \\
10 / 18 \\
10 / 19 \\
10 / 22\end{array}$ & $\begin{array}{l}25 \\
27 \\
28 \\
29 \\
32\end{array}$ & $\begin{array}{r}80 \\
120 \\
132 \\
144 \\
176\end{array}$ & $\begin{array}{ll}12 / & 3 \\
12 / & 5 \\
12 / & 7 \\
12 / & 8 \\
12 / & 9\end{array}$ & $\begin{array}{l}74 \\
76 \\
78 \\
79 \\
80\end{array}$ & $\begin{array}{l}178 \\
190 \\
200 \\
195 \\
200\end{array}$ \\
\hline $\begin{array}{l}10 / 23 \\
10 / 24 \\
10 / 25 \\
10 / 26 \\
10 / 29\end{array}$ & $\begin{array}{l}33 \\
34 \\
35 \\
36 \\
39\end{array}$ & $\begin{array}{l}164 \\
156 \\
150 \\
152 \\
114\end{array}$ & $\begin{array}{l}12 / 10 \\
12 / 11 \\
12 / 12 \\
12 / 13 \\
12 / 14\end{array}$ & $\begin{array}{l}81 \\
82 \\
83 \\
84 \\
85\end{array}$ & $\begin{array}{l}196 \\
195 \\
186 \\
184 \\
184\end{array}$ \\
\hline $\begin{array}{l}10 / 30 \\
10 / 31 \\
11 / 2 \\
11 / 5 \\
11 / 7\end{array}$ & $\begin{array}{l}40 \\
41 \\
43 \\
46 \\
48\end{array}$ & $\begin{array}{l}120 \\
158 \\
134 \\
170 \\
152\end{array}$ & $\begin{array}{l}12 / 17 \\
12 / 18 \\
12 / 19 \\
12 / 20 \\
12 / 21\end{array}$ & $\begin{array}{l}88 \\
89 \\
90 \\
91 \\
92\end{array}$ & $\begin{array}{l}186 \\
186 \\
196 \\
224 \\
210\end{array}$ \\
\hline
\end{tabular}


Caisson-B Outflow Rate (Cont.)

\begin{tabular}{|c|c|c|c|c|c|}
\hline Date & $\begin{array}{l}\text { Days } \\
\text { elapsed }\end{array}$ & $\begin{array}{l}\text { outflow } \\
\text { (ml/min) }\end{array}$ & Date & $\begin{array}{l}\text { Days } \\
\text { elapsed }\end{array}$ & $\begin{array}{l}\text { Outflow } \\
\text { (ml/min) }\end{array}$ \\
\hline $\begin{array}{l}12 / 22 \\
12 / 24 \\
12 / 26 \\
12 / 27 \\
12 / 28\end{array}$ & $\begin{array}{l}93 \\
95 \\
97 \\
98 \\
99\end{array}$ & $\begin{array}{l}204 \\
204 \\
210 \\
208 \\
198\end{array}$ & $\begin{array}{ll}2 / & 1 \\
2 / & 6 \\
2 / & 8 \\
2 / & 9 \\
2 / 10\end{array}$ & $\begin{array}{l}134 \\
139 \\
141 \\
142 \\
143\end{array}$ & $\begin{array}{r}210 \\
210 \\
84 \\
88 \\
140\end{array}$ \\
\hline $\begin{array}{l}12 / 29 \\
12 / 31 \\
1 / 2 / 85 \\
1 / 3 \\
1 / 4\end{array}$ & $\begin{array}{l}100 \\
102 \\
104 \\
105 \\
106\end{array}$ & $\begin{array}{l}200 \\
174 \\
216 \\
208 \\
198\end{array}$ & $\begin{array}{l}2 / 11 \\
2 / 12 \\
2 / 13 \\
2 / 14 \\
2 / 15\end{array}$ & $\begin{array}{l}144 \\
145 \\
146 \\
147 \\
148\end{array}$ & $\begin{array}{l}194 \\
195 \\
188 \\
196 \\
200\end{array}$ \\
\hline $\begin{array}{l}1 / 5 \\
1 / 7 \\
1 / 8 \\
1 / 9 \\
1 / 10\end{array}$ & $\begin{array}{l}107 \\
109 \\
110 \\
111 \\
112\end{array}$ & $\begin{array}{l}192 \\
205 \\
200 \\
180 \\
186\end{array}$ & $\begin{array}{l}2 / 16 \\
2 / 18 \\
2 / 19 \\
2 / 20 \\
2 / 21\end{array}$ & $\begin{array}{l}149 \\
151 \\
152 \\
153 \\
154\end{array}$ & $\begin{array}{l}200 \\
172 \\
186 \\
186 \\
188\end{array}$ \\
\hline $\begin{array}{l}1 / 11 \\
1 / 12 \\
1 / 14 \\
1 / 16 \\
1 / 18\end{array}$ & $\begin{array}{l}113 \\
114 \\
126 \\
118 \\
120\end{array}$ & $\begin{array}{l}212 \\
214 \\
192 \\
190 \\
202\end{array}$ & $\begin{array}{l}2 / 22 \\
2 / 23 \\
2 / 25 \\
2 / 26 \\
2 / 27\end{array}$ & $\begin{array}{l}155 \\
156 \\
1.58 \\
159 \\
160\end{array}$ & $\begin{array}{l}196 \\
204 \\
215 \\
208 \\
200\end{array}$ \\
\hline $\begin{array}{l}1 / 21 \\
1 / 23 \\
1 / 25 \\
1 / 28 \\
1 / 30\end{array}$ & $\begin{array}{l}123 \\
125 \\
127 \\
130 \\
132\end{array}$ & $\begin{array}{l}210 \\
190 \\
204 \\
190 \\
170\end{array}$ & $\begin{array}{l}2 / 28 \\
3 / 2 \\
3 / 2 \\
3 / 4 \\
3 / 5\end{array}$ & $\begin{array}{l}161 \\
162 \\
163 \\
165 \\
i 66\end{array}$ & $\begin{array}{l}205 \\
208 \\
200 \\
206 \\
214\end{array}$ \\
\hline
\end{tabular}


Caisson-B Outlfow Rate (Cont.)

\begin{tabular}{|c|c|c|c|c|c|}
\hline Date & $\begin{array}{l}\text { Days } \\
\text { elapsed }\end{array}$ & $\begin{array}{l}\text { outflow } \\
\text { (ml/min) }\end{array}$ & Date & $\begin{array}{c}\text { Days } \\
\text { elapsed }\end{array}$ & $\begin{array}{l}\text { Outflow } \\
\text { (ml/min) }\end{array}$ \\
\hline $\begin{array}{l}3 / 6 \\
3 / 7 \\
3 / 8 \\
3 / 11 \\
3 / 13\end{array}$ & $\begin{array}{l}167 \\
158 \\
169 \\
172 \\
173\end{array}$ & $\begin{array}{l}216 \\
210 \\
200 \\
200 \\
224\end{array}$ & $\begin{array}{ll}5 / & 6 \\
5 / & 7 \\
5 / & 8 \\
5 / 10 \\
5 / 13\end{array}$ & $\begin{array}{l}227 \\
228 \\
229 \\
231 \\
234\end{array}$ & $\begin{array}{r}110 \\
80 \\
76 \\
70 \\
36\end{array}$ \\
\hline $\begin{array}{l}3 / 15 \\
3 / 18 \\
3 / 2.2 \\
3 / 25 \\
4 / 17\end{array}$ & $\begin{array}{l}175 \\
178 \\
182 \\
185 \\
208\end{array}$ & $\begin{array}{l}214 \\
212 \\
250 \\
250 \\
210\end{array}$ & $\begin{array}{l}5 / 15 \\
5 / 17 \\
5 / 20 \\
5 / 21 \\
5 / 22\end{array}$ & $\begin{array}{l}236 \\
238 \\
241 \\
242 \\
243\end{array}$ & $\begin{array}{l}36 \\
34 \\
28 \\
25 \\
22\end{array}$ \\
\hline $\begin{array}{l}4 / 18 \\
4 / 19 \\
4 / 20 \\
4 / 21 \\
4 / 22\end{array}$ & $\begin{array}{l}209 \\
210 \\
211 \\
212 \\
213\end{array}$ & $\begin{array}{l}200 \\
186 \\
215 \\
240 \\
236\end{array}$ & $\begin{array}{l}5 / 23 \\
5 / 24 \\
5 / 28 \\
5 / 30 \\
6 / 4\end{array}$ & $\begin{array}{l}244 \\
245 \\
249 \\
251 \\
256\end{array}$ & $\begin{array}{c}20 \\
22 \\
20 \\
17.5 \\
14\end{array}$ \\
\hline $\begin{array}{l}4 / 23 \\
4 / 24 \\
4 / 25 \\
4 / 26 \\
4 / 28\end{array}$ & $\begin{array}{l}214 \\
215 \\
216 \\
217 \\
219\end{array}$ & $\begin{array}{l}240 \\
230 \\
192 \\
219 \\
214\end{array}$ & $\begin{array}{l}6 / 12 \\
6 / 17 \\
6 / 19 \\
6 / 20 \\
6 / 21\end{array}$ & $\begin{array}{l}264 \\
269 \\
271 \\
272 \\
273\end{array}$ & $\begin{array}{l}8.0 \\
7.5 \\
7.0 \\
7.5 \\
7.5\end{array}$ \\
\hline $\begin{array}{l}4 / 29 \\
4 / 30 \\
5 / 2 \\
5 / 2 \\
5 / 3\end{array}$ & $\begin{array}{l}220 \\
221 \\
222 \\
223 \\
224\end{array}$ & $\begin{array}{l}224 \\
230 \\
206 \\
180 \\
140\end{array}$ & $\begin{array}{l}6 / 22 \\
6 / 23 \\
6 / 25 \\
6 / 26 \\
6 / 28\end{array}$ & $\begin{array}{l}274 \\
275 \\
277 \\
278 \\
280\end{array}$ & $\begin{array}{l}7.5 \\
8.0 \\
6.0 \\
6.0 \\
4.0\end{array}$ \\
\hline
\end{tabular}


Caisson-B Outflow Rate (Cont.)

\begin{tabular}{|c|c|c|c|c|c|}
\hline Date & $\begin{array}{l}\text { Days } \\
\text { elapsed }\end{array}$ & $\begin{array}{l}\text { outflow } \\
\text { (ml/min) }\end{array}$ & Date & $\begin{array}{l}\text { Days } \\
\text { elapsed }\end{array}$ & $\begin{array}{l}\text { outflow } \\
\text { (ml/min) }\end{array}$ \\
\hline $\begin{array}{l}6 / 29 \\
6 / 30 \\
7 / \\
7 / 2 \\
7 / 3\end{array}$ & $\begin{array}{l}281 \\
282 \\
283 \\
284 \\
285\end{array}$ & $\begin{array}{l}5.6 \\
6.4 \\
5.0 \\
5.0 \\
4.0\end{array}$ & $\begin{array}{l}7 / 29 \\
7 / 31 \\
8 / 1 \\
8 / 4 \\
8 / 5\end{array}$ & $\begin{array}{l}311 \\
313 \\
314 \\
317 \\
318\end{array}$ & $\begin{array}{l}200 \\
197 \\
208 \\
174 \\
206\end{array}$ \\
\hline $\begin{array}{l}7 / 6 \\
7 / 7 \\
7 / 8 \\
7 / 9 \\
7 / 10\end{array}$ & $\begin{array}{l}288 \\
289 \\
290 \\
291 \\
292\end{array}$ & $\begin{array}{l}6.0 \\
4.0 \\
4.0 \\
4.0 \\
4.0\end{array}$ & $\begin{array}{l}8 / 7 \\
8 / 9 \\
8 / 12 \\
8 / 14 \\
8 / 16\end{array}$ & $\begin{array}{l}320 \\
322 \\
325 \\
327 \\
329\end{array}$ & $\begin{array}{l}210 \\
204 \\
184 \\
160 \\
198\end{array}$ \\
\hline $\begin{array}{l}7 / 11 \\
7 / 12 \\
7 / 13 \\
7 / 14 \\
7 / 15\end{array}$ & $\begin{array}{l}293 \\
294 \\
295 \\
296 \\
297\end{array}$ & $\begin{array}{l}4.1 \\
4.0 \\
4.0 \\
4.0 \\
3.5\end{array}$ & $\begin{array}{l}8 / 19 \\
8 / 21 \\
8 / 23 \\
8 / 26 \\
8 / 28\end{array}$ & $\begin{array}{l}332 \\
334 \\
336 \\
339 \\
341\end{array}$ & $\begin{array}{l}204 \\
216 \\
218 \\
214 \\
214\end{array}$ \\
\hline $\begin{array}{l}7 / 16 \\
7 / 17 \\
7 / 18 \\
7 / 20 \\
7 / 21\end{array}$ & $\begin{array}{l}298 \\
299 \\
300 \\
302 \\
303\end{array}$ & $\begin{array}{r}3.0 \\
3.0 \\
3.5 \\
4.0 \\
22\end{array}$ & $\begin{array}{l}8 / 30 \\
9 / 4 \\
9 / 11 \\
9 / 16 \\
9 / 18\end{array}$ & $\begin{array}{l}343 \\
348 \\
355 \\
360 \\
362\end{array}$ & $\begin{array}{l}206 \\
200 \\
210 \\
196 \\
206\end{array}$ \\
\hline $\begin{array}{l}7 / 22 \\
7 / 23 \\
7 / 24 \\
7 / 26 \\
7 / 28\end{array}$ & $\begin{array}{l}304 \\
305 \\
306 \\
308 \\
310\end{array}$ & $\begin{array}{r}30 \\
85 \\
158 \\
180 \\
196\end{array}$ & $\begin{array}{r}9 / 20 \\
9 / 30 \\
10 / 2 \\
10 / 3 \\
10 / 4\end{array}$ & $\begin{array}{l}364 \\
374 \\
376 \\
377 \\
378\end{array}$ & $\begin{array}{l}214 \\
200 \\
184 \\
184 \\
200\end{array}$ \\
\hline
\end{tabular}


TABLE 4

VERTICAL TEMPERATURE PROFILES

INSIDE CAISSON B

(Thermistors located $30 \mathrm{~cm}$ from edge of caisson walls.)

\begin{tabular}{|c|c|c|c|c|c|c|c|}
\hline Date & Time & $\begin{array}{l}\text { Air } \\
\text { (12) }\end{array}$ & $\begin{array}{c}\text { Terface } \\
\text { (1) }\end{array}$ & $\begin{array}{l}\text { aperatu } \\
0.43 \mathrm{~m} \\
(2)\end{array}$ & $\begin{array}{c}\text { ce }\left({ }^{\circ} \mathrm{c}\right) \\
1.19 \mathrm{~m} \\
(3)\end{array}$ & $\begin{array}{c}1.94 \mathrm{~m} \\
(4)\end{array}$ & $\begin{array}{c}2.71 \mathrm{~m} \\
(5)\end{array}$ \\
\hline $\begin{array}{l}11 / 15 / 84 \\
11 / 16 / 84 \\
11 / 16 / 84 \\
11 / 17 / 84 \\
11 / 17 / 84\end{array}$ & $\begin{array}{l}1445 \\
0715 \\
0900 \\
0700 \\
1900\end{array}$ & $\begin{array}{l}7.0 \\
5.0 \\
8.8 \\
6.0 \\
6.8\end{array}$ & $\begin{array}{l}7.0 \\
5.3 \\
6.2 \\
6.7 \\
7.0\end{array}$ & $\begin{array}{l}8.3 \\
8.1 \\
8.5 \\
8.0 \\
8.0\end{array}$ & $\begin{array}{l}9.3 \\
9.0 \\
9.0 \\
9.0 \\
8.8\end{array}$ & $\begin{array}{r}10.3 \\
10.0 \\
10.0 \\
10.0 \\
9.8\end{array}$ & $\begin{array}{l}11.0 \\
10.6 \\
11.5 \\
10.5 \\
10.3\end{array}$ \\
\hline $\begin{array}{l}11 / 18 / 84 \\
11 / 18 / 84 \\
11 / 19 / 84 \\
11 / 20 / 84 \\
11 / 21 / 84\end{array}$ & $\begin{array}{l}0745 \\
2022 \\
0900 \\
0700 \\
0900\end{array}$ & $\begin{array}{l}6.0 \\
6.7 \\
5.0 \\
3.5 \\
6.3\end{array}$ & $\begin{array}{l}6.2 \\
7.0 \\
5.2 \\
4.0 \\
6.0\end{array}$ & $\begin{array}{l}7.7 \\
7.2 \\
7.0 \\
7.2 \\
7.5\end{array}$ & $\begin{array}{l}8.2 \\
8.0 \\
7.8 \\
8.0 \\
8.1\end{array}$ & $\begin{array}{l}9.2 \\
9.0 \\
8.7 \\
8.8 \\
9.0\end{array}$ & $\begin{array}{r}10.0 \\
7.4 \\
9.2 \\
9.5 \\
9.8\end{array}$ \\
\hline $\begin{array}{l}11 / 22 / 84 \\
11 / 23 / 84 \\
11 / 24 / 84 \\
11 / 26 / 84 \\
11 / 27 / 84\end{array}$ & $\begin{array}{l}0645 \\
0650 \\
0730 \\
0800 \\
1400\end{array}$ & $\begin{array}{l}5.0 \\
6.0 \\
6.5 \\
1.0 \\
5.3\end{array}$ & $\begin{array}{l}5.3 \\
5.0 \\
6.9 \\
0.6 \\
5.0\end{array}$ & $\begin{array}{l}7.6 \\
8.0 \\
8.3 \\
7.0 \\
6.0\end{array}$ & $\begin{array}{l}8.3 \\
8.7 \\
9.0 \\
8.2 \\
7.8\end{array}$ & $\begin{array}{l}9.1 \\
9.5 \\
9.9 \\
9.1 \\
8.8\end{array}$ & $\begin{array}{r}9.9 \\
10.1 \\
10.3 \\
10.0 \\
9.5\end{array}$ \\
\hline $\begin{array}{l}11 / 28 / 84 \\
11 / 29 / 84 \\
11 / 30 / 84 \\
12 / 3 / 84 \\
12 / 4 / 84\end{array}$ & $\begin{array}{l}0900 \\
0830 \\
0845 \\
0800 \\
0800\end{array}$ & $\begin{array}{l}3.5 \\
8.8 \\
4.2 \\
5.0 \\
4.8\end{array}$ & $\begin{array}{l}6.1 \\
5.0 \\
4.4 \\
5.5 \\
6.0\end{array}$ & $\begin{array}{l}6.0 \\
6.0 \\
6.0 \\
5.9 \\
5.9\end{array}$ & $\begin{array}{l}7.1 \\
7.1 \\
6.8 \\
6.0 \\
5.9\end{array}$ & $\begin{array}{l}8.2 \\
8.2 \\
8.0 \\
7.2 \\
6.7\end{array}$ & $\begin{array}{l}9.0 \\
9.0 \\
8.9 \\
8.0 \\
7.4\end{array}$ \\
\hline $\begin{array}{ll}12 / & 5 / 84 \\
12 / & 6 / 84 \\
12 / & 7 / 84 \\
12 / & 8 / 84 \\
12 / & 9 / 84\end{array}$ & $\begin{array}{l}0800 \\
1130 \\
0900 \\
0900 \\
1030\end{array}$ & $\begin{array}{l}5.2 \\
7.0 \\
7.9 \\
7.5 \\
7.5\end{array}$ & $\begin{array}{l}5.7 \\
6.2 \\
6.0 \\
5.9 \\
6.9\end{array}$ & $\begin{array}{l}5.2 \\
5.8 \\
6.0 \\
5.9 \\
6.5\end{array}$ & $\begin{array}{l}5.3 \\
5.7 \\
5.7 \\
5.8 \\
6.6\end{array}$ & $\begin{array}{l}5.6 \\
6.3 \\
6.1 \\
6.0 \\
6.4\end{array}$ & $\begin{array}{l}7.0 \\
7.1 \\
7.0 \\
6.9 \\
7.2\end{array}$ \\
\hline $\begin{array}{l}12 / 10 / 84 \\
12 / 11 / 84 \\
12 / 12 / 84 \\
12 / 13 / 84 \\
12 / 17 / 84\end{array}$ & $\begin{array}{l}0900 \\
0900 \\
0830 \\
0945 \\
0930\end{array}$ & $\begin{array}{l}5.7 \\
5.8 \\
4.8 \\
4.3 \\
5.5\end{array}$ & $\begin{array}{l}5.0 \\
6.0 \\
5.0 \\
4.9 \\
2.2\end{array}$ & $\begin{array}{l}6.5 \\
6.7 \\
6.2 \\
6.0 \\
4.0\end{array}$ & $\begin{array}{l}6.3 \\
6.8 \\
6.7 \\
6.2 \\
4.6\end{array}$ & $\begin{array}{l}7.0 \\
7.0 \\
7.0 \\
6.8 \\
5.5\end{array}$ & $\begin{array}{l}7.3 \\
7.6 \\
7.5 \\
7.3 \\
6.0\end{array}$ \\
\hline
\end{tabular}


Vertical Temperature Profiles Inside Caisson B (Cont.)

\begin{tabular}{|c|c|c|c|c|c|c|c|}
\hline Date & Time & $\begin{array}{l}\text { Air } \\
(12)\end{array}$ & $\begin{array}{c}\text { surface } \\
\text { (I) }\end{array}$ & $\begin{array}{c}0.43 \mathrm{~m} \\
(2)\end{array}$ & $\begin{array}{c}\text { dre }\left(^{\circ} \mathrm{C}\right. \\
1.19 \mathrm{~m} \\
(3)\end{array}$ & $\begin{array}{l}1 \\
1.94 \mathrm{~m} \\
\quad(4)\end{array}$ & $\begin{array}{c}2.71 \mathrm{~m} \\
(5)\end{array}$ \\
\hline $\begin{array}{l}12 / 19 / 84 \\
12 / 21 / 84 \\
12 / 26 / 84 \\
12 / 28 / 84 \\
12 / 31 / 84\end{array}$ & $\begin{array}{l}0900 \\
0900 \\
0900 \\
0900 \\
1000\end{array}$ & $\begin{array}{l}3.0 \\
0.7 \\
3.0 \\
3.8 \\
3.9\end{array}$ & $\begin{array}{l}3.0 \\
1.0 \\
2.5 \\
2.1 \\
3.7\end{array}$ & $\begin{array}{l}4.0 \\
4.0 \\
3.1 \\
3.9 \\
4.9\end{array}$ & $\begin{array}{l}4.6 \\
4.2 \\
3.9 \\
4.2 \\
5.0\end{array}$ & $\begin{array}{l}5.5 \\
5.2 \\
5.0 \\
5.1 \\
5.6\end{array}$ & $\begin{array}{l}6.0 \\
6.0 \\
5.5 \\
5.6 \\
6.0\end{array}$ \\
\hline $\begin{array}{l}1 / 3 / 85 \\
1 / 5 / 85 \\
1 / 7 / 85 \\
1 / 9 / 85 \\
1 / 11 / 85\end{array}$ & $\begin{array}{l}0900 \\
0900 \\
1000 \\
0930 \\
1100\end{array}$ & $\begin{array}{l}0.0 \\
1.6 \\
2.0 \\
2.6 \\
2.9\end{array}$ & $\begin{array}{l}0.0 \\
1.3 \\
2.0 \\
2.8 \\
2.5\end{array}$ & $\begin{array}{l}3.2 \\
2.9 \\
3.3 \\
3.6 \\
3.7\end{array}$ & $\begin{array}{l}3.0 \\
3.6 \\
3.9 \\
4.1 \\
4.2\end{array}$ & $\begin{array}{l}4.0 \\
4.6 \\
4.8 \\
4.9 \\
5.0\end{array}$ & $\begin{array}{l}5.6 \\
5.1 \\
5.3 \\
5.3 \\
5.6\end{array}$ \\
\hline $\begin{array}{l}1 / 14 / 85 \\
1 / 16 / 85 \\
1 / 18 / 85 \\
1 / 21 / 85 \\
1 / 23 / 85\end{array}$ & $\begin{array}{l}0900 \\
0830 \\
0830 \\
0840 \\
0845\end{array}$ & $\begin{array}{l}2.8 \\
3.5 \\
3.7 \\
4.1 \\
5.8\end{array}$ & $\begin{array}{l}3.0 \\
4.1 \\
4.4 \\
5.0 \\
6.0\end{array}$ & $\begin{array}{l}3.2 \\
4.0 \\
4.1 \\
5.1 \\
5.0\end{array}$ & $\begin{array}{l}3.9 \\
4.0 \\
4.0 \\
5.0 \\
4.9\end{array}$ & $\begin{array}{l}4.8 \\
4.8 \\
4.6 \\
5.1 \\
5.0\end{array}$ & $\begin{array}{l}5.2 \\
5.2 \\
5.1 \\
5.8 \\
5.3\end{array}$ \\
\hline $\begin{array}{l}1 / 25 / 85 \\
1 / 28 / 85 \\
1 / 30 / 85 \\
2 / 1 / 85 \\
2 / 8 / 85\end{array}$ & $\begin{array}{l}0900 \\
0950 \\
0835 \\
0900 \\
0900\end{array}$ & $\begin{array}{l}7.0 \\
9.8 \\
4.1 \\
0.0 \\
1.1\end{array}$ & $\begin{array}{l}5.7 \\
6.8 \\
5.0 \\
0.1 \\
1.6\end{array}$ & $\begin{array}{l}5.4 \\
6.0 \\
5.0 \\
3.3 \\
1.8\end{array}$ & $\begin{array}{l}5.0 \\
5.5 \\
4.3 \\
3.5 \\
1.8\end{array}$ & $\begin{array}{l}5.0 \\
5.5 \\
4.4 \\
4.0 \\
2.2\end{array}$ & $\begin{array}{l}5.4 \\
6.0 \\
5.0 \\
4.4 \\
3.0\end{array}$ \\
\hline $\begin{array}{l}2 / 11 / 85 \\
2 / 14 / 85 \\
2 / 18 / 85 \\
2 / 20 / 85 \\
2 / 22 / 85\end{array}$ & $\begin{array}{l}0900 \\
0830 \\
0900 \\
0845 \\
0845\end{array}$ & $\begin{array}{l}0.9 \\
2.6 \\
6.0 \\
5.5 \\
6.1\end{array}$ & $\begin{array}{l}1.0 \\
3.0 \\
6.0 \\
5.6 \\
6.3\end{array}$ & $\begin{array}{l}3.0 \\
4.6 \\
5.8 \\
6.0 \\
6.1\end{array}$ & $\begin{array}{l}2.9 \\
4.0 \\
5.0 \\
5.3 \\
6.0\end{array}$ & $\begin{array}{l}3.1 \\
4.2 \\
5.0 \\
5.2 \\
5.6\end{array}$ & $\begin{array}{l}3.9 \\
4.7 \\
5.1 \\
5.5 \\
6.8\end{array}$ \\
\hline $\begin{array}{l}2 / 25 / 85 \\
2 / 27 / 85 \\
3 / 8 / 85 \\
3 / 12 / 85 \\
3 / 19 / 85\end{array}$ & $\begin{array}{l}0845 \\
0835 \\
0830\end{array}$ & $\begin{array}{l}4.0 \\
4.9 \\
5.5 \\
4.5 \\
5.6\end{array}$ & $\begin{array}{l}4.0 \\
5.0 \\
5.8 \\
5.0 \\
5.5\end{array}$ & $\begin{array}{l}5.0 \\
5.2 \\
5.6 \\
5.1 \\
5.6\end{array}$ & $\begin{array}{l}4.7 \\
4.9 \\
5.3 \\
4.8 \\
5.0\end{array}$ & $\begin{array}{l}4.8 \\
4.9 \\
5.2 \\
4.7 \\
4.9\end{array}$ & $\begin{array}{l}5.0 \\
5.0 \\
5.2 \\
5.0 \\
5.1\end{array}$ \\
\hline
\end{tabular}

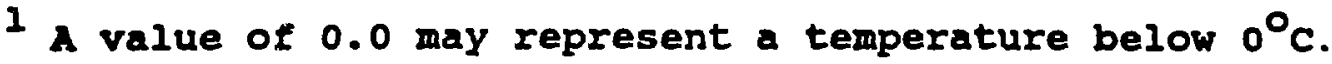


Vertical Temperature Profiles Inside Caisson B (Cont.)

\begin{tabular}{|c|c|c|c|c|c|c|c|}
\hline \multirow[b]{2}{*}{ Date } & \multirow[b]{2}{*}{ Time } & \multicolumn{6}{|c|}{ Temperature $\left({ }^{\circ} \mathrm{C}\right)$} \\
\hline & & $\begin{array}{l}\text { Air } \\
(12)\end{array}$ & $\begin{array}{c}\text { Surface } \\
\text { (I) }\end{array}$ & $\begin{array}{l}0.43 \mathrm{~m} \\
(2)\end{array}$ & $\begin{array}{l}1.19 \mathrm{~m} \\
\text { (3) }\end{array}$ & $\begin{array}{l}1.94 \mathrm{~m} \\
(4)\end{array}$ & $\begin{array}{c}2.71 \mathrm{~m} \\
(5)\end{array}$ \\
\hline $\begin{array}{l}3 / 25 / 85 \\
3 / 27 / 85 \\
3 / 29 / 85 \\
4 / 1 / 85 \\
4 / 6 / 85\end{array}$ & & $\begin{array}{l}6.0 \\
5.2 \\
4.0 \\
7.0\end{array}$ & $\begin{array}{l}6.0 \\
4.0 \\
6.7\end{array}$ & $\begin{array}{l}6.0 \\
6.3 \\
7.1 \\
5.0 \\
7.6\end{array}$ & $\begin{array}{l}5.3 \\
6.0 \\
6.9 \\
4.9 \\
7.0\end{array}$ & $\begin{array}{l}5.4 \\
6.9 \\
6.5 \\
5.0 \\
6.3\end{array}$ & $\begin{array}{l}5.3 \\
6.2 \\
6.2 \\
5.0 \\
6.1\end{array}$ \\
\hline $\begin{array}{l}4 / 7 / 85 \\
4 / 8 / 85 \\
4 / 12 / 85 \\
4 / 15 / 85 \\
4 / 18 / 85\end{array}$ & & $\begin{array}{l}8.5 \\
7.0 \\
7.5 \\
12.5 \\
11.9\end{array}$ & $\begin{array}{r}8.0 \\
7.5 \\
7.6 \\
11.5 \\
11.8\end{array}$ & $\begin{array}{r}8.2 \\
8.3 \\
8.4 \\
11.0 \\
11.1\end{array}$ & $\begin{array}{r}7.5 \\
7.5 \\
7.7 \\
10.0 \\
11.0\end{array}$ & $\begin{array}{r}7.0 \\
7.0 \\
7.1 \\
8.7 \\
10.0\end{array}$ & $\begin{array}{l}6.8 \\
6.9 \\
7.2 \\
8.0 \\
9.1\end{array}$ \\
\hline $\begin{array}{l}4 / 20 / 85 \\
4 / 21 / 85 \\
4 / 22 / 85 \\
4 / 24 / 85 \\
4 / 29 / 85\end{array}$ & & $\begin{array}{l}10.0 \\
8.0 \\
8.1 \\
7.0\end{array}$ & $\begin{array}{r}7.9 \\
10.0 \\
8.0 \\
8.0 \\
7.1\end{array}$ & $\begin{array}{r}8.3 \\
10.5 \\
9.9 \\
9.0 \\
8.8\end{array}$ & $\begin{array}{r}11.7 \\
10.0 \\
9.8 \\
9.0 \\
8.4\end{array}$ & $\begin{array}{r}10.6 \\
9.5 \\
9.0 \\
8.9 \\
8.3\end{array}$ & $\begin{array}{l}9.7 \\
9.0 \\
8.7 \\
8.2 \\
8.1\end{array}$ \\
\hline $\begin{array}{l}5 / 2 / 85 \\
5 / 6 / 85 \\
5 / 8 / 85 \\
5 / 10 / 85 \\
5 / 25 / 85\end{array}$ & & $\begin{array}{l}9.0 \\
11.5 \\
12.1 \\
12.3 \\
8.5\end{array}$ & $\begin{array}{r}9.0 \\
11.5 \\
12.0 \\
12.0 \\
8.5\end{array}$ & $\begin{array}{l}10.0 \\
11.5 \\
12.8 \\
13.0 \\
10.5\end{array}$ & $\begin{array}{r}9.2 \\
10.5 \\
11.6 \\
11.8 \\
10.0\end{array}$ & $\begin{array}{r}9.0 \\
9.5 \\
10.8 \\
10.9 \\
9.8\end{array}$ & $\begin{array}{r}8.9 \\
9.0 \\
10.2 \\
10.2 \\
9.4\end{array}$ \\
\hline $\begin{array}{l}5 / 17 / 85 \\
5 / 21 / 85 \\
5 / 22 / 85 \\
5 / 28 / 85 \\
5 / 30 / 85\end{array}$ & & $\begin{array}{l}12.2 \\
9.7 \\
11.0 \\
11.5 \\
13.5\end{array}$ & $\begin{array}{r}11.4 \\
9.4 \\
12.0 \\
11.5 \\
13.0\end{array}$ & $\begin{array}{l}11.9 \\
10.5 \\
10.5 \\
12.6 \\
14.5\end{array}$ & $\begin{array}{l}11.0 \\
10.0 \\
10.0 \\
11.4 \\
13.0\end{array}$ & $\begin{array}{r}10.6 \\
9.9 \\
9.9 \\
10.8 \\
12.0\end{array}$ & $\begin{array}{r}10.1 \\
9.5 \\
9.5 \\
10.1 \\
11.0\end{array}$ \\
\hline $\begin{array}{l}6 / 4 / 85 \\
6 / 6 / 85 \\
6 / 10 / 85 \\
6 / 11 / 85 \\
6 / 12 / 85\end{array}$ & & $\begin{array}{l}12.0 \\
13.0 \\
15.5 \\
14.0 \\
16.0\end{array}$ & $\begin{array}{l}12.5 \\
12.5 \\
15.5 \\
14.5 \\
15.0\end{array}$ & $\begin{array}{l}14.0 \\
12.0 \\
15.5 \\
16.0 \\
14.9\end{array}$ & $\begin{array}{l}12.9 \\
11.5 \\
14.0 \\
14.5 \\
13.8\end{array}$ & $\begin{array}{l}11.9 \\
10.9 \\
12.9 \\
13.1 \\
12.5\end{array}$ & $\begin{array}{l}11.1 \\
10.2 \\
12.0 \\
12.4 \\
12.0\end{array}$ \\
\hline
\end{tabular}


Vertical Temperature Profiles Inside Caisson B (Cont.)

\begin{tabular}{|c|c|c|c|c|c|c|c|}
\hline \multirow[b]{2}{*}{ Date } & \multirow[b]{2}{*}{ Time } & \multicolumn{6}{|c|}{ Temperature $\left({ }^{\circ} \mathrm{C}\right)$} \\
\hline & & $\begin{array}{l}\text { Air } \\
\text { (12) }\end{array}$ & $\begin{array}{c}\text { Surface } \\
\text { (1) }\end{array}$ & $\begin{array}{c}0.43 \mathrm{~m} \\
(2)\end{array}$ & $\begin{array}{c}1.19 \mathrm{~m} \\
(3)\end{array}$ & $\begin{array}{c}1.94 \mathrm{~m} \\
(4)\end{array}$ & $\begin{array}{c}2.71 \mathrm{~m} \\
(5)\end{array}$ \\
\hline $\begin{array}{l}6 / 14 / 85 \\
6 / 17 / 85 \\
6 / 19 / 85 \\
6 / 21 / 85 \\
6 / 24 / 85\end{array}$ & & $\begin{array}{l}12.2 \\
16.5 \\
15.2 \\
16.5 \\
17.0\end{array}$ & $\begin{array}{l}16.2 \\
16.0 \\
15.0 \\
16.5 \\
17.0\end{array}$ & $\begin{array}{l}15.5 \\
17.0 \\
16.0 \\
17.0 \\
17.8\end{array}$ & $\begin{array}{l}14.2 \\
15.2 \\
14.9 \\
15.5 \\
16.3\end{array}$ & $\begin{array}{l}13.1 \\
13.9 \\
13.7 \\
14.5 \\
15.0\end{array}$ & $\begin{array}{l}12.2 \\
13.0 \\
13.0 \\
13.5 \\
14.0\end{array}$ \\
\hline $\begin{array}{l}6 / 26 / 85 \\
6 / 29 / 85 \\
7 / 2 / 85 \\
7 / 4 / 85 \\
7 / 6 / 85\end{array}$ & & $\begin{array}{l}17.0 \\
16.1 \\
16.9 \\
20.0 \\
17.6\end{array}$ & $\begin{array}{l}16.5 \\
15.9 \\
17.0 \\
18.5 \\
17.8\end{array}$ & $\begin{array}{l}17.5 \\
17.2 \\
18.2 \\
18.0 \\
19.3\end{array}$ & $\begin{array}{l}16.0 \\
16.0 \\
17.0 \\
17.0 \\
18.0\end{array}$ & $\begin{array}{l}15.0 \\
15.0 \\
16.0 \\
16.0 \\
17.0\end{array}$ & $\begin{array}{l}14.0 \\
14.1 \\
15.0 \\
15.0 \\
15.8\end{array}$ \\
\hline $\begin{array}{l}7 / 8 / 85 \\
7 / 10 / 85 \\
7 / 12 / 85 \\
7 / 13 / 85 \\
7 / 16 / 85\end{array}$ & & $\begin{array}{l}19.0 \\
18.5 \\
19.8 \\
22.5 \\
19.5\end{array}$ & $\begin{array}{l}18.9 \\
18.0 \\
19.9 \\
22.0 \\
19.0\end{array}$ & $\begin{array}{l}20.0 \\
20.0 \\
20.3 \\
21.0 \\
20.5\end{array}$ & $\begin{array}{l}18.6 \\
18.8 \\
19.1 \\
19.0 \\
19.5\end{array}$ & $\begin{array}{l}17.2 \\
17.5 \\
18.0 \\
18.0 \\
18.0\end{array}$ & $\begin{array}{l}16.1 \\
16.5 \\
16.9 \\
16.5 \\
16.9\end{array}$ \\
\hline $\begin{array}{l}7 / 18 / 85 \\
7 / 20 / 85 \\
7 / 23 / 85 \\
7 / 25 / 85 \\
7 / 27 / 85\end{array}$ & & $\begin{array}{l}19.0 \\
21.0 \\
19.5 \\
18.1 \\
21.0\end{array}$ & $\begin{array}{l}19.5 \\
20.5 \\
19.0 \\
17.8 \\
20.2\end{array}$ & $\begin{array}{l}20.0 \\
20.0 \\
20.5 \\
19.5 \\
19.5\end{array}$ & $\begin{array}{l}19.5 \\
19.0 \\
19.0 \\
18.8 \\
18.8\end{array}$ & $\begin{array}{l}18.5 \\
17.8 \\
18.0 \\
17.8 \\
18.0\end{array}$ & $\begin{array}{l}16.5 \\
16.5 \\
17.0 \\
17.0 \\
17.0\end{array}$ \\
\hline $\begin{array}{l}7 / 29 / 85 \\
7 / 31 / 85 \\
8 / 2 / 85 \\
8 / 7 / 85 \\
8 / 9 / 85\end{array}$ & & $\begin{array}{l}18.8 \\
17.2 \\
17.5 \\
18.1 \\
18.1\end{array}$ & $\begin{array}{l}18.8 \\
17.1 \\
17.9 \\
18.0 \\
18.2\end{array}$ & $\begin{array}{l}18.7 \\
18.7 \\
19.4 \\
19.8 \\
20.1\end{array}$ & $\begin{array}{l}18.2 \\
18.0 \\
18.6 \\
18.9 \\
19.0\end{array}$ & $\begin{array}{l}17.8 \\
17.4 \\
18.0 \\
18.1 \\
18.5\end{array}$ & $\begin{array}{l}17.0 \\
17.0 \\
17.4 \\
17.6 \\
17.9\end{array}$ \\
\hline $\begin{array}{l}8 / 12 / 85 \\
8 / 14 / 85 \\
8 / 16 / 85 \\
8 / 19 / 85 \\
8 / 21 / 85\end{array}$ & & $\begin{array}{l}19.4 \\
19.7 \\
19.3 \\
27.5 \\
17.6\end{array}$ & $\begin{array}{l}18.5 \\
18.8 \\
18.8 \\
17.2 \\
18.0\end{array}$ & $\begin{array}{l}18.6 \\
18.6 \\
19.0 \\
18.9 \\
19.2\end{array}$ & $\begin{array}{l}18.0 \\
18.0 \\
18.4 \\
18.0 \\
18.4\end{array}$ & $\begin{array}{l}18.5 \\
17.5 \\
18.0 \\
17.8 \\
18.0\end{array}$ & $\begin{array}{l}17.0 \\
17.0 \\
17.5 \\
17.2 \\
17.5\end{array}$ \\
\hline
\end{tabular}


Vertical Temperature Profiles Inside Caisson B (Cont.)

\begin{tabular}{|c|c|c|c|c|c|c|c|}
\hline \multirow[b]{2}{*}{ Date } & \multirow[b]{2}{*}{ Time } & \multicolumn{6}{|c|}{ Temperature $\left({ }^{\circ} \mathrm{C}\right)$} \\
\hline & & $\begin{array}{l}\text { Air } \\
\text { (12) }\end{array}$ & $\begin{array}{l}\text { Surface } \\
\text { (1) }\end{array}$ & $\begin{array}{l}0.43 \mathrm{~m} \\
(2)\end{array}$ & $1.19 \mathrm{~m}$ & $\begin{array}{c}1.94 \mathrm{~m} \\
(4)\end{array}$ & $\begin{array}{c}2.71 \mathrm{~m} \\
(5)\end{array}$ \\
\hline $\begin{array}{l}8 / 23 / 85 \\
8 / 26 / 85 \\
8 / 30 / 85 \\
9 / 3 / 85 \\
9 / 6 / 85\end{array}$ & & $\begin{array}{l}20.0 \\
18.9 \\
18.5 \\
18.0 \\
18.5\end{array}$ & $\begin{array}{l}19.5 \\
18.7 \\
18.5 \\
17.1 \\
17.5\end{array}$ & $\begin{array}{l}20.0 \\
20.0 \\
19.5 \\
20.2 \\
20.0\end{array}$ & $\begin{array}{l}18.8 \\
19.0 \\
19.0 \\
19.1 \\
19.2\end{array}$ & $\begin{array}{l}18.2 \\
18.2 \\
18.2 \\
18.6 \\
18.5\end{array}$ & $\begin{array}{l}17.7 \\
18.0 \\
17.8 \\
18.0 \\
18.0\end{array}$ \\
\hline $\begin{array}{l}9 / 13 / 85 \\
9 / 16 / 85 \\
9 / 18 / 85 \\
9 / 20 / 85\end{array}$ & & $\begin{array}{l}18.5 \\
15.5 \\
16.1 \\
13.8\end{array}$ & $\begin{array}{l}17.0 \\
15.0 \\
16.0 \\
14.0\end{array}$ & $\begin{array}{l}20.0 \\
18.0 \\
16.8 \\
16.1\end{array}$ & $\begin{array}{l}19.0 \\
17.5 \\
16.2 \\
16.0\end{array}$ & $\begin{array}{l}18.3 \\
17.0 \\
16.2 \\
16.1\end{array}$ & $\begin{array}{l}17.8 \\
17.0 \\
16.1 \\
16.1\end{array}$ \\
\hline
\end{tabular}


TABLE 5

HORIZONTAI TEMPERATURE PROFILES ACROSS CAISSON B

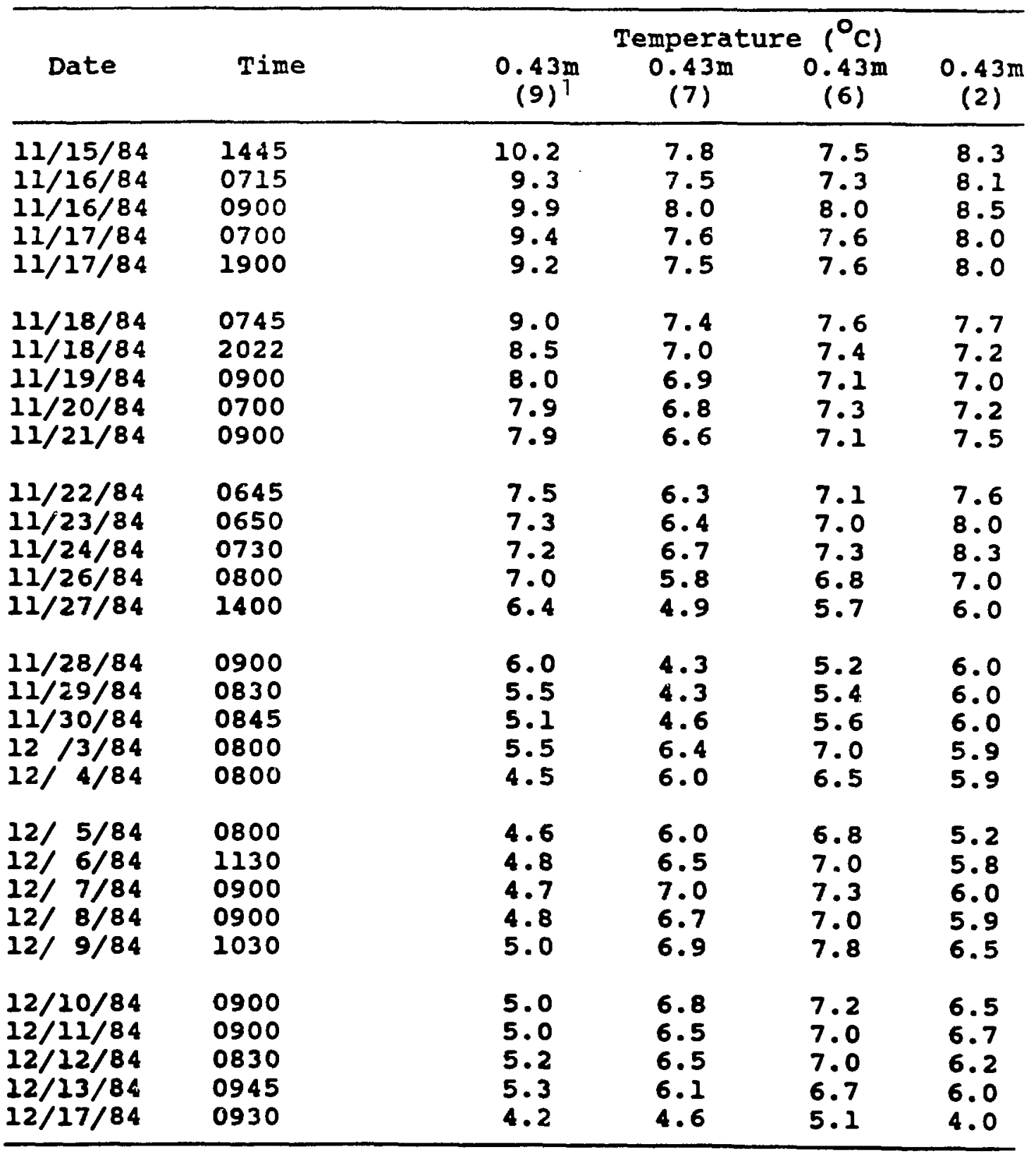

I Data collected at stations $9,7,6$, and 2, which are located $0.70 \mathrm{~m}$ outside of the caisson, and $2.70,1.5$, and $0.3 \mathrm{~m}$ from the caisson wall, respectively. 
Horizontal Temperature Profiles Across Caisson B (Cont.)

\begin{tabular}{|c|c|c|c|c|c|}
\hline \multirow[b]{2}{*}{ Date } & \multirow[b]{2}{*}{ Time } & \multicolumn{4}{|c|}{ Temperature $\left({ }^{\circ} \mathrm{C}\right)$} \\
\hline & & $\begin{array}{c}0.43 \mathrm{~m} \\
(9)^{1}\end{array}$ & $\begin{array}{c}0.43 \mathrm{~m} \\
(7)\end{array}$ & $\begin{array}{c}0.43 \mathrm{~m} \\
(6)\end{array}$ & $\begin{array}{c}0.43 \mathrm{~m} \\
(2)\end{array}$ \\
\hline $\begin{array}{l}12 / 19 / 84 \\
12 / 21 / 84 \\
12 / 26 / 84 \\
12 / 28 / 84 \\
12 / 31 / 84\end{array}$ & $\begin{array}{l}0900 \\
0900 \\
0900 \\
0900 \\
1000\end{array}$ & $\begin{array}{l}4.0 \\
3.6 \\
3.1 \\
3.4 \\
4.0\end{array}$ & $\begin{array}{l}4.4 \\
4.0 \\
3.5 \\
3.2 \\
5.0\end{array}$ & $\begin{array}{l}5.0 \\
4.9 \\
4.0 \\
4.5 \\
5.5\end{array}$ & $\begin{array}{l}4.0 \\
4.0 \\
3.1 \\
3.9 \\
4.9\end{array}$ \\
\hline $\begin{array}{ll}1 / & 3 / 85 \\
1 / & 5 / 85 \\
1 / & 7 / 85 \\
1 / & 9 / 85 \\
1 / 11 / 85\end{array}$ & $\begin{array}{l}0900 \\
0900 \\
1000 \\
0930 \\
1100\end{array}$ & $\begin{array}{l}3.0 \\
2.9 \\
2.6 \\
2.5 \\
3.7\end{array}$ & $\begin{array}{l}3.2 \\
3.0 \\
3.1 \\
3.1 \\
3.3\end{array}$ & $\begin{array}{l}3.9 \\
3.3 \\
3.4 \\
3.5 \\
4.0\end{array}$ & $\begin{array}{l}3.2 \\
2.9 \\
3.3 \\
3.6 \\
3.7\end{array}$ \\
\hline $\begin{array}{l}1 / 14 / 85 \\
1 / 16 / 85 \\
1 / 18 / 85 \\
1 / 21 / 85 \\
1 / 23 / 85\end{array}$ & $\begin{array}{l}0900 \\
0830 \\
0830 \\
0840 \\
0845\end{array}$ & $\begin{array}{l}2.5 \\
2.3 \\
2.2 \\
3.0 \\
2.5\end{array}$ & $\begin{array}{l}3.8 \\
4.5 \\
5.0 \\
6.0 \\
5.9\end{array}$ & $\begin{array}{l}4.0 \\
5.1 \\
5.6 \\
6.9 \\
6.6\end{array}$ & $\begin{array}{l}3.2 \\
4.0 \\
4.1 \\
5.1 \\
5.0\end{array}$ \\
\hline $\begin{array}{l}1 / 25 / 85 \\
1 / 28 / 85 \\
1 / 30 / 85 \\
2 / 1 / 85 \\
2 / 8 / 85\end{array}$ & $\begin{array}{l}0900 \\
0950 \\
0835 \\
0900 \\
0900\end{array}$ & $\begin{array}{l}2.6 \\
3.0 \\
3.0 \\
2.9 \\
2.0\end{array}$ & $\begin{array}{l}6.1 \\
6.9 \\
7.6 \\
5.8 \\
3.6\end{array}$ & $\begin{array}{l}7.0 \\
7.5 \\
7.2 \\
6.9 \\
4.2\end{array}$ & $\begin{array}{l}5.4 \\
6.0 \\
5.0 \\
3.3 \\
1.8\end{array}$ \\
\hline $\begin{array}{l}2 / 11 / 85 \\
2 / 14 / 85 \\
2 / 18 / 85 \\
2 / 20 / 85 \\
2 / 22 / 85\end{array}$ & $\begin{array}{l}0900 \\
0830 \\
0900 \\
0845 \\
0845\end{array}$ & $\begin{array}{l}2.9 \\
1.7 \\
2.7 \\
3.1 \\
3.6\end{array}$ & $\begin{array}{l}4.2 \\
4.8 \\
5.8 \\
6.0 \\
6.2\end{array}$ & $\begin{array}{l}5.5 \\
5.8 \\
6.6 \\
7.0 \\
7.1\end{array}$ & $\begin{array}{l}3.0 \\
4.6 \\
5.8 \\
6.0 \\
6.1\end{array}$ \\
\hline $\begin{array}{l}2 / 25 / 85 \\
2 / 27 / 85 \\
3 / 8 / 85 \\
3 / 12 / 85 \\
3 / 19 / 85\end{array}$ & $\begin{array}{l}0845 \\
0835 \\
0830\end{array}$ & $\begin{array}{l}3.0 \\
3.1 \\
5.5 \\
8.0 \\
4.1\end{array}$ & $\begin{array}{l}6.0 \\
6.2 \\
6.4 \\
7.2 \\
6.4\end{array}$ & $\begin{array}{l}7.0 \\
7.2 \\
7.0 \\
7.2 \\
7.1\end{array}$ & $\begin{array}{l}5.0 \\
5.2 \\
5.6 \\
7.1 \\
5.6\end{array}$ \\
\hline
\end{tabular}


Horizontal Temperature Profiles Across Caisson $B$

\begin{tabular}{|c|c|c|c|c|c|}
\hline Date & Time & $\begin{array}{c}0.43 \mathrm{~m} \\
(9)^{1}\end{array}$ & $\begin{array}{c}\text { Tempera } \\
0.43 \mathrm{~m} \\
(7)\end{array}$ & $\begin{array}{c}\left.0 .{ }^{0} \mathrm{C}\right) \\
0.43 \mathrm{~m} \\
(6)\end{array}$ & $\begin{array}{c}0.43 \mathrm{~m} \\
(2)\end{array}$ \\
\hline $\begin{array}{l}3 / 25 / 85 \\
3 / 27 / 85 \\
3 / 29 / 85 \\
4 / 1 / 85 \\
4 / 6 / 85\end{array}$ & & $\begin{array}{l}7.0 \\
7.8 \\
8.0 \\
6.0 \\
8.3\end{array}$ & $\begin{array}{l}7.0 \\
7.1 \\
7.2 \\
6.2 \\
8.1\end{array}$ & $\begin{array}{l}7.0 \\
7.1 \\
7.2 \\
7.0 \\
8.1\end{array}$ & $\begin{array}{l}6.0 \\
6.9 \\
7.1 \\
5.0 \\
7.6\end{array}$ \\
\hline $\begin{array}{l}4 / 7 / 85 \\
4 / 8 / 85 \\
4 / 12 / 85 \\
4 / 15 / 85 \\
4 / 18 / 85\end{array}$ & & $\begin{array}{r}9.0 \\
9.7 \\
9.5 \\
11.5 \\
13.0\end{array}$ & $\begin{array}{r}9.0 \\
9.0 \\
9.2 \\
11.5 \\
12.2\end{array}$ & $\begin{array}{r}8.9 \\
9.0 \\
9.1 \\
11.7 \\
12.8\end{array}$ & $\begin{array}{r}8.2 \\
8.3 \\
8.4 \\
11.0 \\
11.1\end{array}$ \\
\hline $\begin{array}{l}4 / 20 / 85 \\
4 / 21 / 85 \\
4 / 22 / 85 \\
4 / 24 / 85 \\
4 / 29 / 85\end{array}$ & & $\begin{array}{l}13.0 \\
12.0 \\
11.8 \\
11.0 \\
10.4\end{array}$ & $\begin{array}{r}12.0 \\
11.5 \\
11.0 \\
10.1 \\
9.7\end{array}$ & $\begin{array}{l}12.7 \\
11.5 \\
11.2 \\
10.4 \\
10.0\end{array}$ & $\begin{array}{r}11.7 \\
10.5 \\
9.9 \\
9.0 \\
8.8\end{array}$ \\
\hline $\begin{array}{ll}5 / 2 / 85 \\
5 / & 6 / 85 \\
5 / & 8 / 85 \\
5 / 10 / 85 \\
5 / 15 / 85\end{array}$ & & $\begin{array}{l}11.0 \\
13.0 \\
14.5 \\
15.5 \\
14.4\end{array}$ & $\begin{array}{l}10.3 \\
11.5 \\
11.9 \\
13.0 \\
12.0\end{array}$ & $\begin{array}{l}10.2 \\
11.0 \\
12.0 \\
12.0 \\
11.0\end{array}$ & $\begin{array}{l}10.0 \\
11.5 \\
12.8 \\
13.0 \\
10.5\end{array}$ \\
\hline $\begin{array}{l}5 / 17 / 85 \\
5 / 21 / 85 \\
5 / 22 / 85 \\
5 / 28 / 85 \\
5 / 30 / 85\end{array}$ & & $\begin{array}{l}25.0 \\
13.6 \\
13.0 \\
14.3 \\
16.0\end{array}$ & $\begin{array}{l}12.6 \\
12.0 \\
11.8 \\
12.9 \\
14.0\end{array}$ & $\begin{array}{l}11 \cdot 3 \\
11 \cdot 2 \\
11 \cdot 1 \\
12.0 \\
13.5\end{array}$ & $\begin{array}{l}11.9 \\
10.5 \\
10.5 \\
12.6 \\
14.5\end{array}$ \\
\hline $\begin{array}{l}6 / 4 / 85 \\
6 / 6 / 85 \\
6 / 10 / 85 \\
6 / 11 / 85 \\
6 / 12 / 85\end{array}$ & & $\begin{array}{l}17.1 \\
16.0 \\
18.0 \\
19.0 \\
18.3\end{array}$ & $\begin{array}{l}14.2 \\
13.5 \\
16.0 \\
16.3 \\
15.5\end{array}$ & $\begin{array}{l}13.1 \\
12.5 \\
14.7 \\
15.2 \\
14.5\end{array}$ & $\begin{array}{l}14.0 \\
12.0 \\
15.5 \\
16.0 \\
14.9\end{array}$ \\
\hline
\end{tabular}


Horizontal Temperature Profiles Across Caisson B (Cont.)

\begin{tabular}{|c|c|c|c|c|c|}
\hline \multirow[b]{2}{*}{ Date } & \multirow[b]{2}{*}{ Time } & \multicolumn{4}{|c|}{ Temperature $\left({ }^{0} \mathrm{C}\right)$} \\
\hline & & $\begin{array}{c}0.43 \mathrm{~m} \\
(9)^{\prime}\end{array}$ & $\begin{array}{c}0.43 \mathrm{~m} \\
(7)\end{array}$ & $\begin{array}{c}0.43 \mathrm{~m} \\
(6)\end{array}$ & $\begin{array}{c}0.43 \mathrm{~m} \\
(2)\end{array}$ \\
\hline $\begin{array}{l}6 / 14 / 85 \\
6 / 17 / 85 \\
6 / 19 / 85 \\
6 / 21 / 85 \\
6 / 24 / 85\end{array}$ & & $\begin{array}{l}13.6 \\
19.6 \\
19.0 \\
28.5 \\
19.6\end{array}$ & $\begin{array}{l}16.0 \\
17.0 \\
16.5 \\
17.0 \\
17.5\end{array}$ & $\begin{array}{l}15.0 \\
16.0 \\
15.8 \\
16.0 \\
16.9\end{array}$ & $\begin{array}{l}15.5 \\
17.0 \\
16.0 \\
17.0 \\
17.8\end{array}$ \\
\hline $\begin{array}{l}6 / 26 / 85 \\
6 / 29 / 85 \\
7 / 2 / 85 \\
7 / 4 / 85 \\
7 / 6 / 85\end{array}$ & & $\begin{array}{l}18.5 \\
19.5 \\
20.8 \\
21.0 \\
22.0\end{array}$ & $\begin{array}{l}17.5 \\
17.2 \\
18.0 \\
18 \cdot 0 \\
29.0\end{array}$ & $\begin{array}{l}16.5 \\
16.8 \\
17.3 \\
17.5 \\
18.0\end{array}$ & $\begin{array}{l}17.5 \\
17.2 \\
18.2 \\
18.0 \\
19.3\end{array}$ \\
\hline $\begin{array}{l}7 / 8 / 85 \\
7 / 10 / 85 \\
7 / 12 / 85 \\
7 / 13 / 85 \\
7 / 16 / 85\end{array}$ & & $\begin{array}{l}23.0 \\
23.0 \\
23.8 \\
24.0 \\
24.0\end{array}$ & $\begin{array}{l}19.8 \\
20.0 \\
20.8 \\
21.0 \\
21.5\end{array}$ & $\begin{array}{l}18.6 \\
18.8 \\
20.0 \\
20.7 \\
21.5\end{array}$ & $\begin{array}{l}20.0 \\
20.0 \\
20.3 \\
21.0 \\
20.5\end{array}$ \\
\hline $\begin{array}{l}7 / 18 / 85 \\
7 / 20 / 85 \\
7 / 23 / 85 \\
7 / 25 / 85 \\
7 / 27 / 85\end{array}$ & & $\begin{array}{l}25 \cdot 0 \\
22 \cdot 5 \\
22 \cdot 0 \\
21 \cdot 8 \\
21 \cdot 5\end{array}$ & $\begin{array}{l}21 \cdot 5 \\
21.0 \\
21.0 \\
20.4 \\
20.0\end{array}$ & $\begin{array}{l}21.0 \\
21.0 \\
21.0 \\
20.8 \\
20.5\end{array}$ & $\begin{array}{l}20.0 \\
20.0 \\
20.5 \\
19.5 \\
19.5\end{array}$ \\
\hline $\begin{array}{l}7 / 29 / 85 \\
7 / 31 / 85 \\
8 / 2 / 85 \\
8 / 7 / 85 \\
8 / 9 / 85\end{array}$ & & $\begin{array}{l}21.4 \\
20.9 \\
21.0 \\
21.0 \\
21.5\end{array}$ & $\begin{array}{l}19.9 \\
19.8 \\
29.8 \\
20.0 \\
20.5\end{array}$ & $\begin{array}{l}20.0 \\
19.5 \\
20.0 \\
20.0 \\
20.5\end{array}$ & $\begin{array}{l}18.7 \\
18.7 \\
19.4 \\
19.8 \\
20.1\end{array}$ \\
\hline $\begin{array}{l}8 / 12 / 85 \\
8 / 14 / 85 \\
8 / 16 / 85 \\
8 / 19 / 85 \\
8 / 21 / 85\end{array}$ & & $\begin{array}{l}21.0 \\
20.9 \\
21.3 \\
22.0 \\
22.0\end{array}$ & $\begin{array}{l}19.5 \\
19.6 \\
20.0 \\
19.9 \\
20.0\end{array}$ & $\begin{array}{l}20.0 \\
19.9 \\
19.9 \\
20.0 \\
20.1\end{array}$ & $\begin{array}{l}18.6 \\
18.6 \\
19.0 \\
18.9 \\
19.2\end{array}$ \\
\hline
\end{tabular}


Horizontal Temperature Profiles Across Caisson B (Cont.)

\begin{tabular}{lcccc}
\hline Date & Time & \multicolumn{5}{c}{ Temperature $\left({ }^{\circ} \mathrm{C}\right)$} \\
& $0.43 \mathrm{~m}$ & $0.43 \mathrm{~m}$ & $0.43 \mathrm{~m}$ & $0.43 \mathrm{~m}$ \\
& $(9)^{1}$ & $(7)$ & $(6)$ & $(2)$ \\
\hline $8 / 23 / 85$ & 22.0 & 20.5 & 20.5 & 20.0 \\
$8 / 26 / 85$ & 22.5 & 21.0 & 21.0 & 20.0 \\
$8 / 30 / 85$ & 22.2 & 20.7 & 20.5 & 19.5 \\
$9 / 3 / 85$ & 23.3 & 21.2 & 21.0 & 20.0 \\
$9 / 6 / 85$ & 23.5 & 21.0 & 21.2 & 20.0 \\
$9 / 13 / 85$ & 23.0 & 20.9 & 20.2 & 20.0 \\
$9 / 16 / 85$ & 21.0 & 19.0 & 18.5 & 18.0 \\
$9 / 18 / 85$ & 19.9 & 18.0 & 17.8 & 16.8 \\
$9 / 20 / 85$ & 3.9 .0 & 17.2 & 17.2 & 16.1 \\
\hline
\end{tabular}


TARLE 6

VERTICAL TEMPERATURE PROFILES

OUTSIDE CAISSON B

(Thermistors located $1.0 \mathrm{~m}$ from edge of caisson wall. )

\begin{tabular}{|c|c|c|c|c|c|}
\hline vate & Time & $\begin{array}{c}\text { Surface } \\
\text { (8) }\end{array}$ & $\begin{array}{c}\text { Temperatu } \\
0.43 \mathrm{~m} \\
(9)\end{array}$ & $\begin{array}{l}\left({ }^{\circ} \mathrm{c}\right) \\
1.0 \mathrm{~m} \\
(10)\end{array}$ & $\begin{array}{l}2.0 \mathrm{~m} \\
(11)\end{array}$ \\
\hline $\begin{array}{l}11 / 15 / 84 \\
11 / 16 / 84 \\
11 / 16 / 84 \\
11 / 17 / 84 \\
11 / 17 / 84\end{array}$ & $\begin{array}{l}1445 \\
0715 \\
0900 \\
0700 \\
1900\end{array}$ & $\begin{array}{r}10.7 \\
3.6 \\
4.0 \\
5.0 \\
4.5\end{array}$ & $\begin{array}{r}10.2 \\
9.3 \\
9.9 \\
9.4 \\
9.2\end{array}$ & $\begin{array}{l}11.3 \\
11.0 \\
11.2 \\
11.2 \\
11.3\end{array}$ & $\begin{array}{l}13.5 \\
13.4 \\
13.7 \\
13.8 \\
13.8\end{array}$ \\
\hline $\begin{array}{l}11 / 18 / 84 \\
11 / 18 / 84 \\
11 / 19 / 84 \\
11 / 20 / 84 \\
11 / 21 / 84\end{array}$ & $\begin{array}{l}0745 \\
2022 \\
0900 \\
0700 \\
0900\end{array}$ & $\begin{array}{l}2.5 \\
4.7 \\
2.0 \\
1.8 \\
1.1\end{array}$ & $\begin{array}{l}9.0 \\
8.5 \\
8.0 \\
7.9 \\
7.9\end{array}$ & $\begin{array}{l}11.0 \\
11.0 \\
10.8 \\
10.6 \\
10.4\end{array}$ & $\begin{array}{l}13.5 \\
13.5 \\
13.1 \\
13.2 \\
13.2\end{array}$ \\
\hline $\begin{array}{l}11 / 22 / 84 \\
11 / 23 / 84 \\
11 / 24 / 84 \\
11 / 26 / 84 \\
11 / 27 / 84\end{array}$ & $\begin{array}{l}0645 \\
0650 \\
0730 \\
0800 \\
1400\end{array}$ & $\begin{array}{l}2.0 \\
2.0 \\
3.2 \\
0.2 \\
0.2\end{array}$ & $\begin{array}{l}7.5 \\
7.3 \\
7.2 \\
7.0 \\
6.4\end{array}$ & $\begin{array}{l}10.3 \\
10.2 \\
10.1 \\
10.0 \\
9.9\end{array}$ & $\begin{array}{l}13.0 \\
13.0 \\
13.0 \\
12.9 \\
13.0\end{array}$ \\
\hline $\begin{array}{l}11 / 28 / 84 \\
11 / 29 / 84 \\
11 / 30 / 84 \\
12 / 3 / 84 \\
12 / 4 / 84\end{array}$ & $\begin{array}{l}0900 \\
0830 \\
0845 \\
0800 \\
0800\end{array}$ & $\begin{array}{l}0.1 \\
0.1 \\
0.1 \\
0.9 \\
0.1\end{array}$ & $\begin{array}{l}6.0 \\
5.5 \\
5.1 \\
5.5 \\
4.5\end{array}$ & $\begin{array}{l}9.5 \\
9.2 \\
9.0 \\
8.6 \\
8.0\end{array}$ & $\begin{array}{l}12.8 \\
12.6 \\
12.8 \\
12.3 \\
11.9\end{array}$ \\
\hline $\begin{array}{ll}12 / & 5 / 84 \\
12 / & 6 / 84 \\
12 / & 7 / 84 \\
12 / & 8 / 84 \\
12 / & 9 / 84\end{array}$ & $\begin{array}{l}0800 \\
1130 \\
0900 \\
0900 \\
1030\end{array}$ & $\begin{array}{l}0.2 \\
0.1 \\
0.1 \\
0.1 \\
2.5\end{array}$ & $\begin{array}{l}4.6 \\
4.8 \\
4.7 \\
4.8 \\
5.0\end{array}$ & $\begin{array}{l}8.0 \\
8.0 \\
7.9 \\
8.0 \\
7.6\end{array}$ & $\begin{array}{l}11.8 \\
11.8 \\
21.8 \\
11.8 \\
11.7\end{array}$ \\
\hline $\begin{array}{l}12 / 10 / 84 \\
12 / 11 / 84 \\
12 / 12 / 84 \\
12 / 13 / 84 \\
12 / 17 / 84\end{array}$ & $\begin{array}{l}0900 \\
0900 \\
0830 \\
0945 \\
0930\end{array}$ & $\begin{array}{l}1.0 \\
3.0 \\
2.0 \\
1.2 \\
0.2\end{array}$ & $\begin{array}{l}5.0 \\
5.0 \\
5.2 \\
5.3 \\
4.2\end{array}$ & $\begin{array}{l}7.5 \\
7.5 \\
7.5 \\
7.5 \\
7.2\end{array}$ & $\begin{array}{l}1 .-2 \\
11.2 \\
11.1 \\
11.1 \\
10.9\end{array}$ \\
\hline
\end{tabular}


Vertical Temperature Profiles Outside Caisson B (Cont.)

\begin{tabular}{|c|c|c|c|c|c|}
\hline \multirow[b]{2}{*}{ Date } & \multirow[b]{2}{*}{ Time } & \multicolumn{4}{|c|}{ Temperature $\left({ }^{\circ} \mathrm{C}\right)^{1}$} \\
\hline & & $\begin{array}{c}\text { Surface } \\
\text { (B) }\end{array}$ & $\begin{array}{c}0.43 \mathrm{~m} \\
(9)\end{array}$ & $\begin{array}{l}2.0 \mathrm{~m} \\
(20)\end{array}$ & $\begin{array}{l}2.0 \mathrm{~m} \\
\text { (il) }\end{array}$ \\
\hline $\begin{array}{l}12 / 19 / 84 \\
12 / 21 / 84 \\
12 / 26 / 84 \\
12 / 28 / 84 \\
12 / 31 / 84\end{array}$ & $\begin{array}{l}0900 \\
0900 \\
0900 \\
0900 \\
1000\end{array}$ & $\begin{array}{l}0.2 \\
0.0 \\
0.0 \\
0.0 \\
0.9\end{array}$ & $\begin{array}{l}4.0 \\
3.6 \\
3.1 \\
3.4 \\
4.0\end{array}$ & $\begin{array}{l}7.0 \\
6.7 \\
6.2 \\
6.0 \\
6.0\end{array}$ & $\begin{array}{l}10.6 \\
10.2 \\
10.1 \\
10.4 \\
9.7\end{array}$ \\
\hline $\begin{array}{ll}1 / 3 / 85 \\
1 / & 5 / 85 \\
1 / 7 / 85 \\
1 / 9 / 85 \\
1 / 11 / 85\end{array}$ & $\begin{array}{l}0900 \\
0900 \\
1000 \\
0930 \\
1100\end{array}$ & $\begin{array}{l}0.0 \\
0.0 \\
0.0 \\
0.0 \\
0.0\end{array}$ & $\begin{array}{l}3.0 \\
2.9 \\
2.6 \\
2.5 \\
3.7\end{array}$ & $\begin{array}{l}5.2 \\
5.3 \\
5.1 \\
5.0 \\
5.2\end{array}$ & $\begin{array}{l}7.6 \\
6.5 \\
8.0 \\
8.0 \\
6.3\end{array}$ \\
\hline $\begin{array}{l}1 / 14 / 85 \\
1 / 16 / 85 \\
1 / 18 / 85 \\
1 / 21 / 85 \\
1 / 23 / 85\end{array}$ & $\begin{array}{l}0900 \\
0830 \\
0830 \\
0840 \\
0845\end{array}$ & $\begin{array}{l}0.0 \\
0.0 \\
0.0 \\
0.0 \\
0.0\end{array}$ & $\begin{array}{l}2.5 \\
2.3 \\
2.2 \\
3.0 \\
2.5\end{array}$ & $\begin{array}{l}5.0 \\
5.0 \\
4.8 \\
5.1 \\
4.9\end{array}$ & $\begin{array}{l}8.0 \\
7.9 \\
7.4 \\
8.0 \\
7.3\end{array}$ \\
\hline $\begin{array}{l}1 / 25 / 85 \\
1 / 28 / 85 \\
1 / 30 / 85 \\
2 / 1 / 85 \\
2 / 8 / 85\end{array}$ & $\begin{array}{l}0900 \\
0950 \\
0835 \\
0900 \\
0900\end{array}$ & $\begin{array}{l}0.0 \\
0.9 \\
0.0 \\
0.0 \\
0.0\end{array}$ & $\begin{array}{l}2.6 \\
3.0 \\
3.0 \\
2.9 \\
2.0\end{array}$ & $\begin{array}{l}4.6 \\
5.0 \\
4.5 \\
4.8 \\
4.2\end{array}$ & $\begin{array}{l}7.6 \\
7.6 \\
7.2 \\
7.3 \\
7.1\end{array}$ \\
\hline $\begin{array}{l}2 / 11 / 85 \\
2 / 14 / 85 \\
2 / 18 / 85 \\
2 / 20 / 85 \\
2 / 22 / 85\end{array}$ & $\begin{array}{l}0900 \\
0830 \\
0900 \\
0845 \\
0845\end{array}$ & $\begin{array}{l}0.0 \\
0.0 \\
1.3 \\
2.0 \\
2.3\end{array}$ & $\begin{array}{l}1.9 \\
1.7 \\
2.7 \\
3.1 \\
3.6\end{array}$ & $\begin{array}{l}4.0 \\
3.9 \\
4.0 \\
4.0 \\
4.0\end{array}$ & $\begin{array}{l}7.1 \\
6.9 \\
6.9 \\
6.8 \\
6.5\end{array}$ \\
\hline $\begin{array}{l}2 / 25 / 85 \\
2 / 27 / 85 \\
3 / 8 / 85 \\
3 / 12 / 85 \\
3 / 19 / 85\end{array}$ & $\begin{array}{l}0845 \\
0835 \\
\text { OB30 }\end{array}$ & $\begin{array}{l}0.1 \\
1.0 \\
3.0 \\
0.1 \\
0.2\end{array}$ & $\begin{array}{l}3.0 \\
3.1 \\
5.5 \\
3.0 \\
4.1\end{array}$ & $\begin{array}{l}4.1 \\
4.1 \\
5.5 \\
4.0 \\
5.0\end{array}$ & $\begin{array}{l}6.4 \\
0.0 \\
0.0 \\
0.0 \\
0.0\end{array}$ \\
\hline
\end{tabular}

I A value of 0.0 may represent a temperature below $0^{\circ} \mathrm{C}$. 
Vertical Temperature Profiles Outside Caisson B (Cont.)

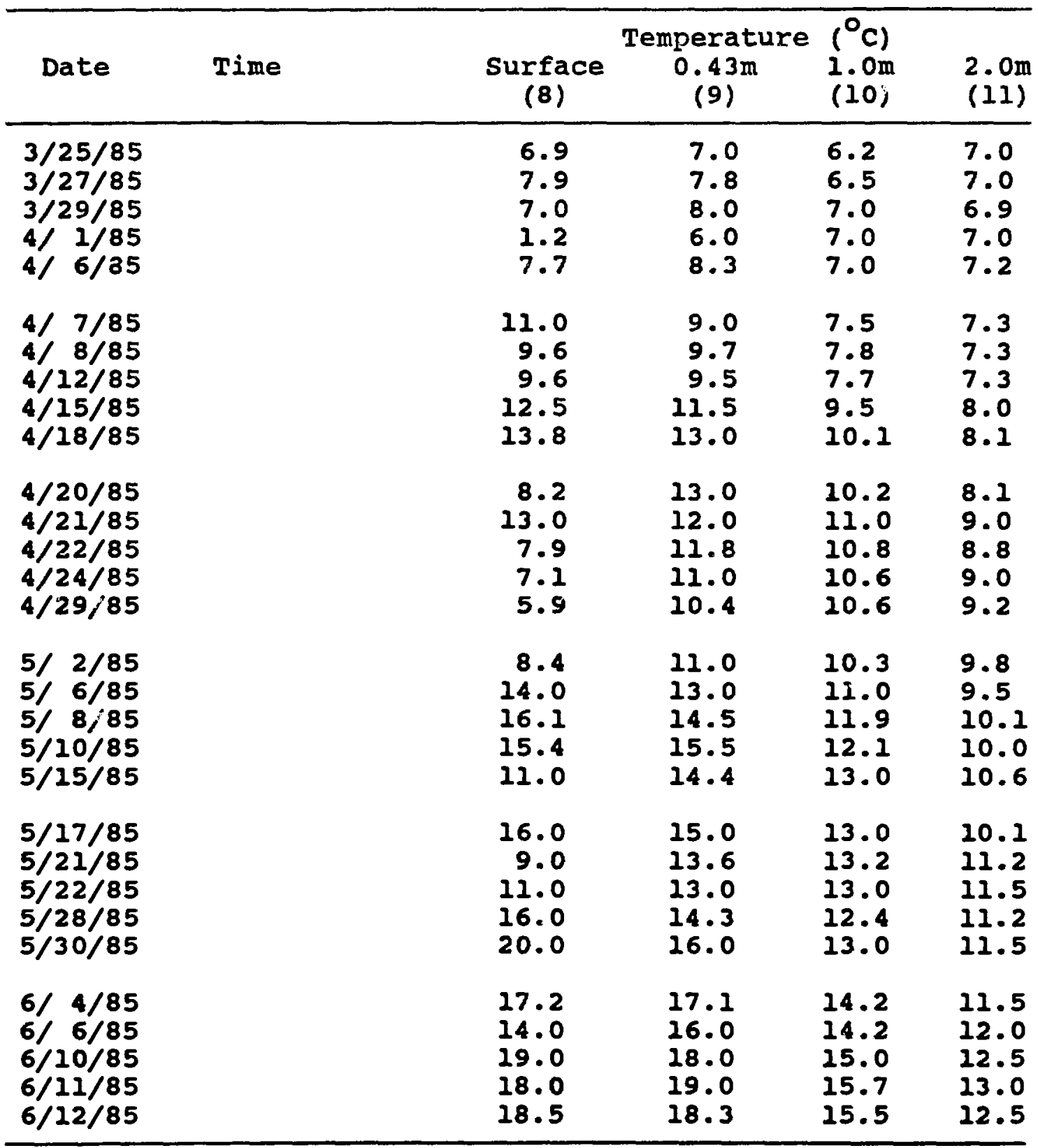


Vertical Temperature Profiles outside Caisson B (Cont.)

\begin{tabular}{|c|c|c|c|c|c|}
\hline Date & Time & $\begin{array}{c}\text { Surface } \\
(8)\end{array}$ & $\begin{array}{c}\text { Temperatu } \\
0.43 \mathrm{~m} \\
(9)\end{array}$ & $\begin{array}{l}\left({ }^{\circ} \mathrm{C}\right) \\
1.0 \mathrm{~m} \\
(10)\end{array}$ & $\begin{array}{l}2.0 \mathrm{~m} \\
\text { (II) }\end{array}$ \\
\hline $\begin{array}{l}6 / 14 / 85 \\
6 / 17 / 85 \\
6 / 19 / 85 \\
6 / 21 / 85 \\
6 / 24 / 85\end{array}$ & & $\begin{array}{l}19.9 \\
20.5 \\
15.0 \\
19.0 \\
18.9\end{array}$ & $\begin{array}{l}13.6 \\
19.6 \\
19.0 \\
18.5 \\
19.6\end{array}$ & $\begin{array}{l}15.9 \\
15.2 \\
16.5 \\
16.5 \\
16.8\end{array}$ & $\begin{array}{l}12.5 \\
13.0 \\
12.9 \\
13.0 \\
13.2\end{array}$ \\
\hline $\begin{array}{l}6 / 26 / 85 \\
6 / 29 / 85 \\
7 / 2 / 85 \\
7 / 4 / 85 \\
7 / 6 / 85\end{array}$ & & $\begin{array}{l}19.0 \\
19.5 \\
20.8 \\
23.0 \\
23.0\end{array}$ & $\begin{array}{l}18.5 \\
19.5 \\
20.8 \\
21.0 \\
22.0\end{array}$ & $\begin{array}{l}16.5 \\
17.0 \\
17.8 \\
18.0 \\
18.5\end{array}$ & $\begin{array}{l}13.5 \\
13.5 \\
14.0 \\
14.0 \\
14.6\end{array}$ \\
\hline $\begin{array}{l}7 / 8 / 85 \\
7 / 10 / 85 \\
7 / 12 / 85 \\
7 / 13 / 85 \\
7 / 16 / 85\end{array}$ & & $\begin{array}{l}24.2 \\
24.0 \\
24.2 \\
27.0 \\
19.0\end{array}$ & $\begin{array}{l}23.0 \\
23.0 \\
23.8 \\
24.0 \\
24.0\end{array}$ & $\begin{array}{l}19.0 \\
19.2 \\
20.0 \\
20.0 \\
21.0\end{array}$ & $\begin{array}{l}15.0 \\
15.0 \\
15.3 \\
15.2 \\
16.0\end{array}$ \\
\hline $\begin{array}{l}7 / 18 / 85 \\
7 / 20 / 85 \\
7 / 23 / 85 \\
7 / 25 / 85 \\
7 / 27 / 85\end{array}$ & & $\begin{array}{l}20.0 \\
21.0 \\
20.0 \\
19.3 \\
23.0\end{array}$ & $\begin{array}{l}25.0 \\
22.5 \\
22.0 \\
21.8 \\
21.5\end{array}$ & $\begin{array}{l}22.0 \\
20.2 \\
20.5 \\
20.3 \\
20.5\end{array}$ & $\begin{array}{l}16.5 \\
16.0 \\
16.5 \\
16.9 \\
17.0\end{array}$ \\
\hline $\begin{array}{l}7 / 29 / 85 \\
7 / 31 / 85 \\
8 / 2 / 85 \\
8 / 7 / 85 \\
8 / 9 / 85\end{array}$ & & $\begin{array}{l}21.0 \\
17.0 \\
18.0 \\
18.8 \\
18.0\end{array}$ & $\begin{array}{l}21.4 \\
20.9 \\
21.0 \\
21.0 \\
21.5\end{array}$ & $\begin{array}{l}20.4 \\
20.2 \\
20.0 \\
19.8 \\
19.9\end{array}$ & $\begin{array}{l}17.2 \\
17.0 \\
17.2 \\
17.5 \\
17.5\end{array}$ \\
\hline $\begin{array}{l}8 / 12 / 85 \\
8 / 14 / 85 \\
8 / 16 / 85 \\
8 / 19 / 85 \\
8 / 21 / 85\end{array}$ & & $\begin{array}{l}19.0 \\
20.8 \\
21.0 \\
17.2 \\
18.8\end{array}$ & $\begin{array}{l}21.0 \\
20.9 \\
21.3 \\
22.0 \\
22.0\end{array}$ & $\begin{array}{l}20.0 \\
20.0 \\
20.0 \\
20.2 \\
20.3\end{array}$ & $\begin{array}{l}17.8 \\
17.8 \\
17.8 \\
17.9 \\
18.0\end{array}$ \\
\hline
\end{tabular}


Vertical Temperature Profiles Outside Caisson B (Cont.)

\begin{tabular}{lccccc}
\hline Date & Time & $\begin{array}{c}\text { Surface } \\
(8)\end{array}$ & $\begin{array}{c}\text { Temperacure } \\
0.43 \mathrm{~m} \\
(9)\end{array}$ & $\begin{array}{l}\left.{ }^{\circ} \mathrm{C}\right) \\
1.0 \mathrm{~m} \\
(10)\end{array}$ & $\begin{array}{c}2 . \text { Om } \\
(11)\end{array}$ \\
\hline $8 / 23 / 85$ & 20.8 & 22.0 & 20.5 & 18.0 \\
$8 / 26 / 85$ & 20.9 & 22.5 & 20.8 & 18.0 \\
$8 / 30 / 85$ & 19.8 & 22.2 & 21.0 & 18.2 \\
$9 / 3 / 85$ & 19.8 & 23.3 & 21.2 & 18.8 \\
$9 / 6 / 85$ & 19.5 & 23.5 & 21.5 & 18.5 \\
$9 / 13 / 85$ & 19.0 & 23.0 & 21.0 & 18.0 \\
$9 / 16 / 85$ & 14.9 & 21.0 & 20.5 & 19.0 \\
$9 / 18 / 85$ & 17.0 & 19.9 & 20.0 & 15.0 \\
$9 / 20 / 85$ & 13.0 & 19.0 & 20.0 & 19.0 \\
\hline
\end{tabular}




\section{A. Tracer Data from Access Port Samplers}

Iodide, bromide, and lithium concentrations are summarized in Figs. 5-28 as a function of time for each of the sampling locations located at access ports for two steady-state and two unsteady-state pulse experiments. Data are presented for pulse experiments where tracer was added: September 20-26, 1984 (unsteady-state pulse 1); December 6-12, 1984 (steady-state pulse 1); February 8-14, 1985 (steady-state pulse 2); and April 8-14, 1985 (unsteady-state pulse 2). During the final pulse experiment, steady-state flow conditions were maintained until April 29, on which date input matrix solution flow was stopped and the caisson was allowed to drain until July 11, 1985, at which time the matrix solution was reinitiated.

In order to present the data in a more comprehensive manner, the iodide tracer data are presented in Figs. 29 and 30 as a function of time and depth for the steady-state pulse experiments. The iodide peak heights and shapes are similar at all six depths in the caisson as would be expected with these flow conditions. The peak iodide concentrations moved uniformly down the caisson from sampling port to sampling port at a rate that varied from 6.3 to $12.5 \mathrm{~cm} \mathrm{~d}^{-1}$, except for the relatively fast movement between the 197- to 271 $\mathrm{cm}$ depths. This abnormally fast migration was probably caused by a tuff layer of minimal compaction at this depth interval, allowing the flow of water and iodide to increase dramatically. (The void ratio of 0.71 found at the $1.56-\mathrm{m}$ depth was considerably larger than that found at any other depth, thus, promoting conductivity.)

Similar iodide tracer data are presented for the two unsteady-state pulse experiments in Figs. 31 and 32. In contrast to the steady-state data (Figs. 29 and 30$)$, the iodide peak heights and shapes are not similar at all six sampling depths in the caisson. Eventually, after the peak iodide 
concentration passes a sampling depth, a small concentration (tail) persists for a long time. In spite of these differences, the iodide peak concentration transport times ranged from 6.3 to $11.0 \mathrm{~cm} \mathrm{~d}^{-1}$, similar to those of the steady-state experiments (Figs. 29 and 30 ).

We also compared the transport of iodide to that of bromide (Fig. 33) and lithium (Fig. 34). Bromide appeared to migrate similarly to iodide (Fig. 33), as would be expected, but lithium and iodide were not similar in their steady-state transport behavior (compare Fig. 34 with Fig. 29, as well as Figs. 11-22). It was expected that lithium would be mobile because of its weak affinity for exchange sites in the presence of the $200 \mathrm{ppm}$ calcium in the inflow solution. However, the lithium traveled about $15 \%$ slower than the iodide in the first steady-state experiment (notice the time axis in Fig. 34 is different from Fig. 29) and exhibited concentrations that changed very slowly relative to iodide, resul:ing in very dissimilar transport when compared with iodide.

The differences in solute transport under steady-state flow conditions were observed to be even more exaggerated under unsteady-state flow conditions. The second unsteady-state pulse, for example, represented an extreme case, as far as variation in solution flow rates, when compared with the first unsteady-state pulse, and the tracer data reflect this difference (Figs. 23-28). Although the volumetric soil water content of the tuff in Caisson B remained consistently at $27-28 \%$ for the first twenty days of the experiment, it decreased to almost $15 \% 60$ days later (Appendix A). During this drainage period it appears that the iodide and bromide migration was enhanced relative to steady-state migration at the $347-\mathrm{cm}$ (Fig. 27) and 421-cm (Fig. 28) sampling depths. In contrast, transport of lithium at these two depths seemed to be dramatically reduced during the (unsteady-state) drainage portion of the 44 
second unsteady-state pulse: experiment relative to steady-state migration. The latter observation is hased on an analysis of the peak tracer concentrations as a function of time at each sampling depth; further data analysis and modeling need to be accomplished to make this point more valid by comparing tracer concentrations as a function of pore volumes of solution being transported through a sampling depth. These observations have very important imp?ications, as far as applying any type of modeling approach to the transport of either nonsorbing (iodide, bromide) or partially-sorbing (lithium) tracers under extreme unsteady-state flow conditions, conditions which would prevail under field conditions.

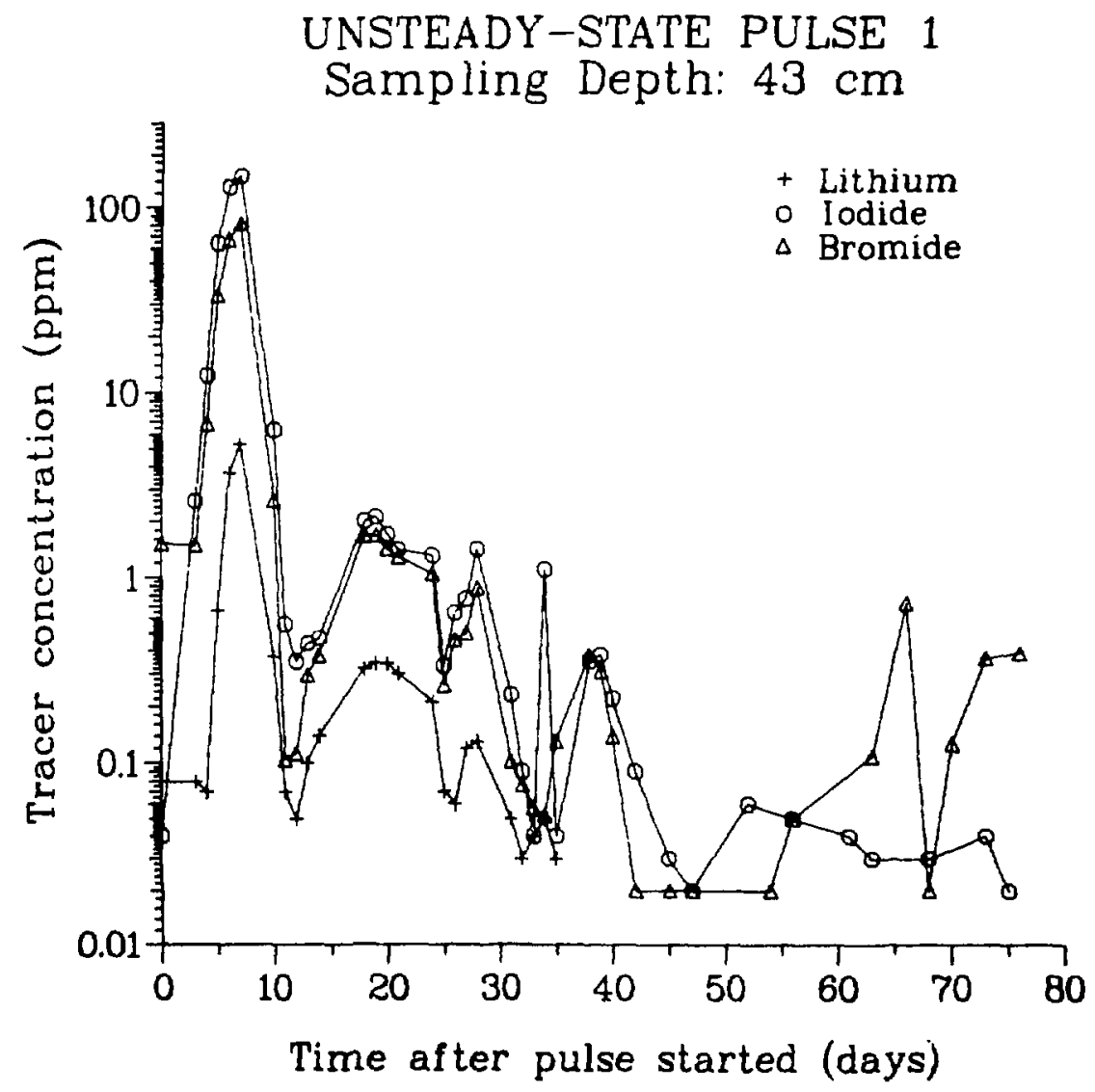

Fig. 5. Iodide, bromide, and lithium concentrations in Caisson B as a function of time after the unsteady-state pulse 1 was started at the $43-\mathrm{cm}$ depth. 


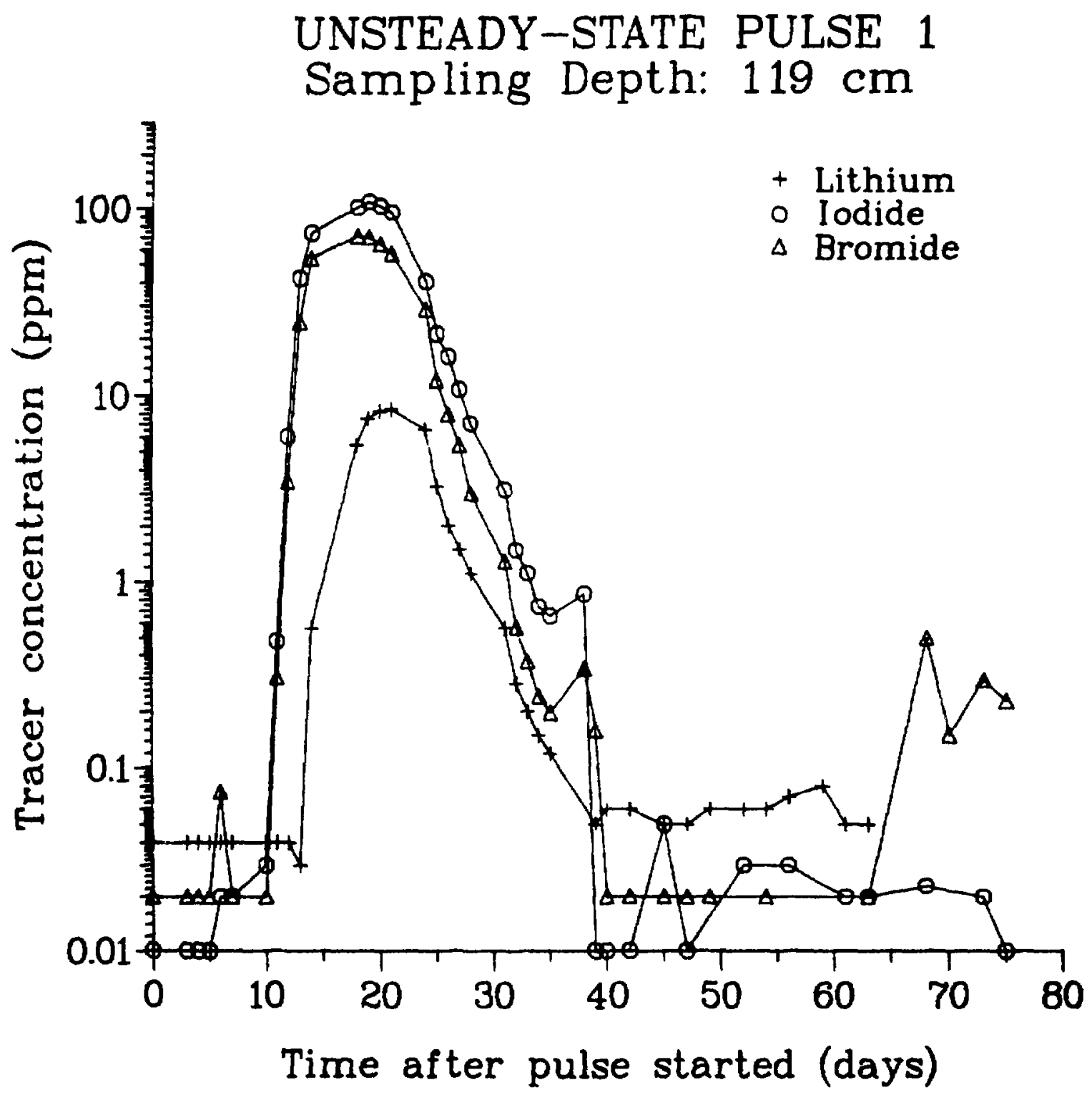

Fig. 6. Iodide, bromide, and lithium concentrations in Caisson B as a function of time after the unsteady-state pulse 1 was started at the $119-\mathrm{cm}$ depth. 


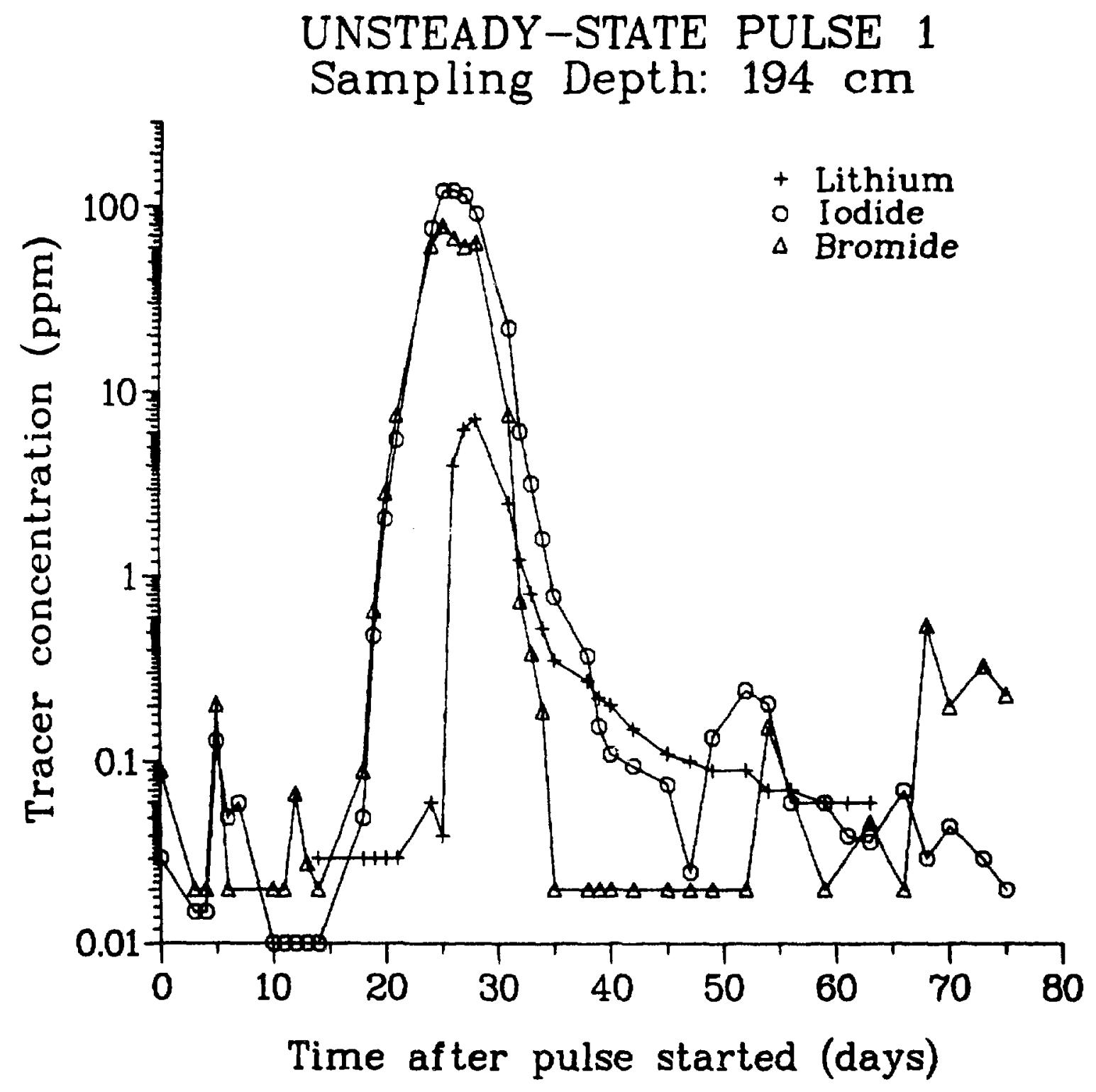

Fig. 7. Iodide, bromide, and lithium concentrations in Caisson B as a function of time after the unsteady-state pulse 1 was started at the 194-cm depth. 


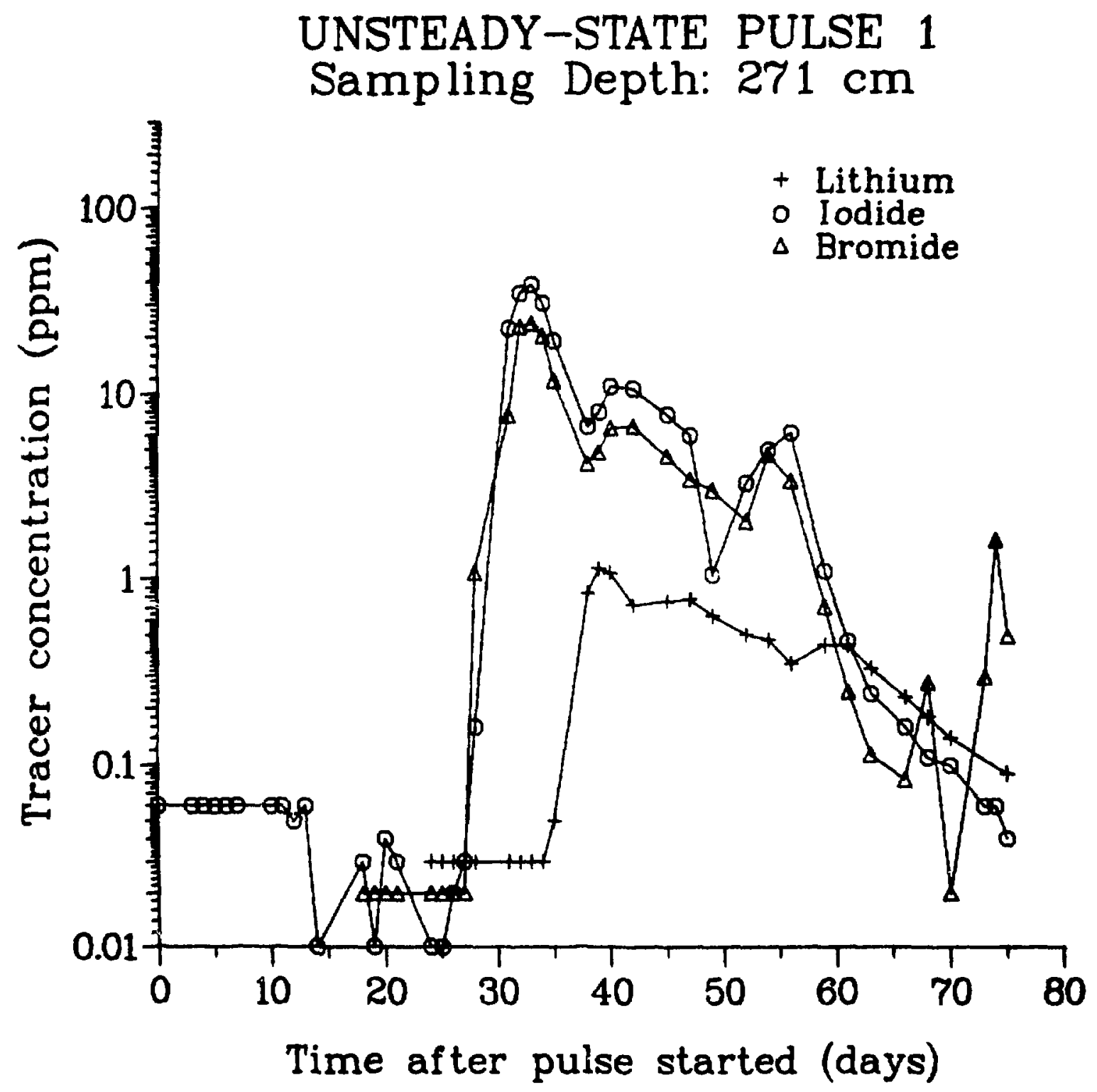

Fig. 8. Iodide, bromide, and lithium concentrations in Caisson B as a function of time after the unsteady-state pulse 1 was started at the 271-cm depth. 


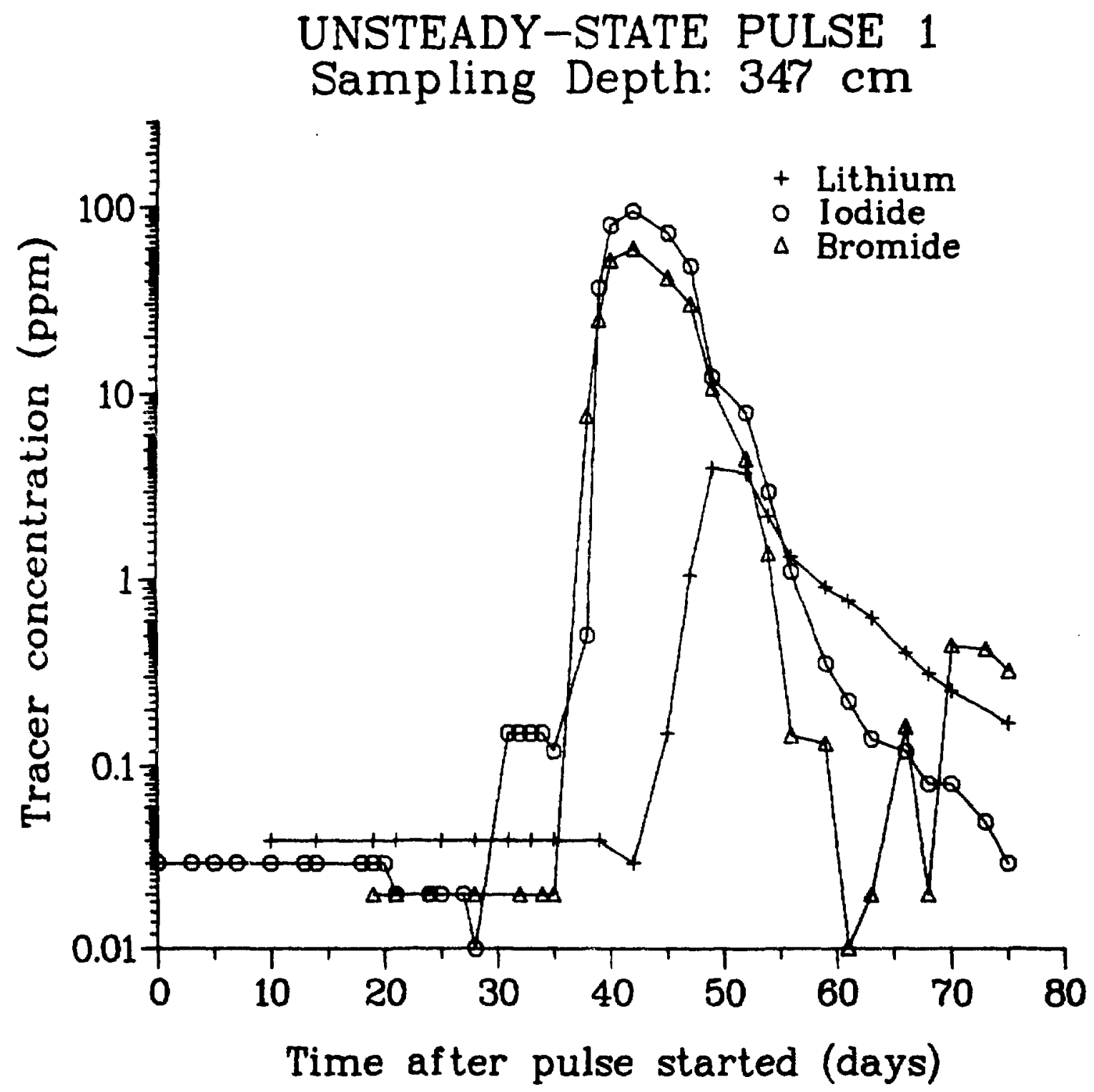

Fig. 9. Iodide, bromide, and lithium concentrations in Caisson B as a function of time after the unsteady-state pulse 1 was started at the $347-\mathrm{cm}$ depth. 


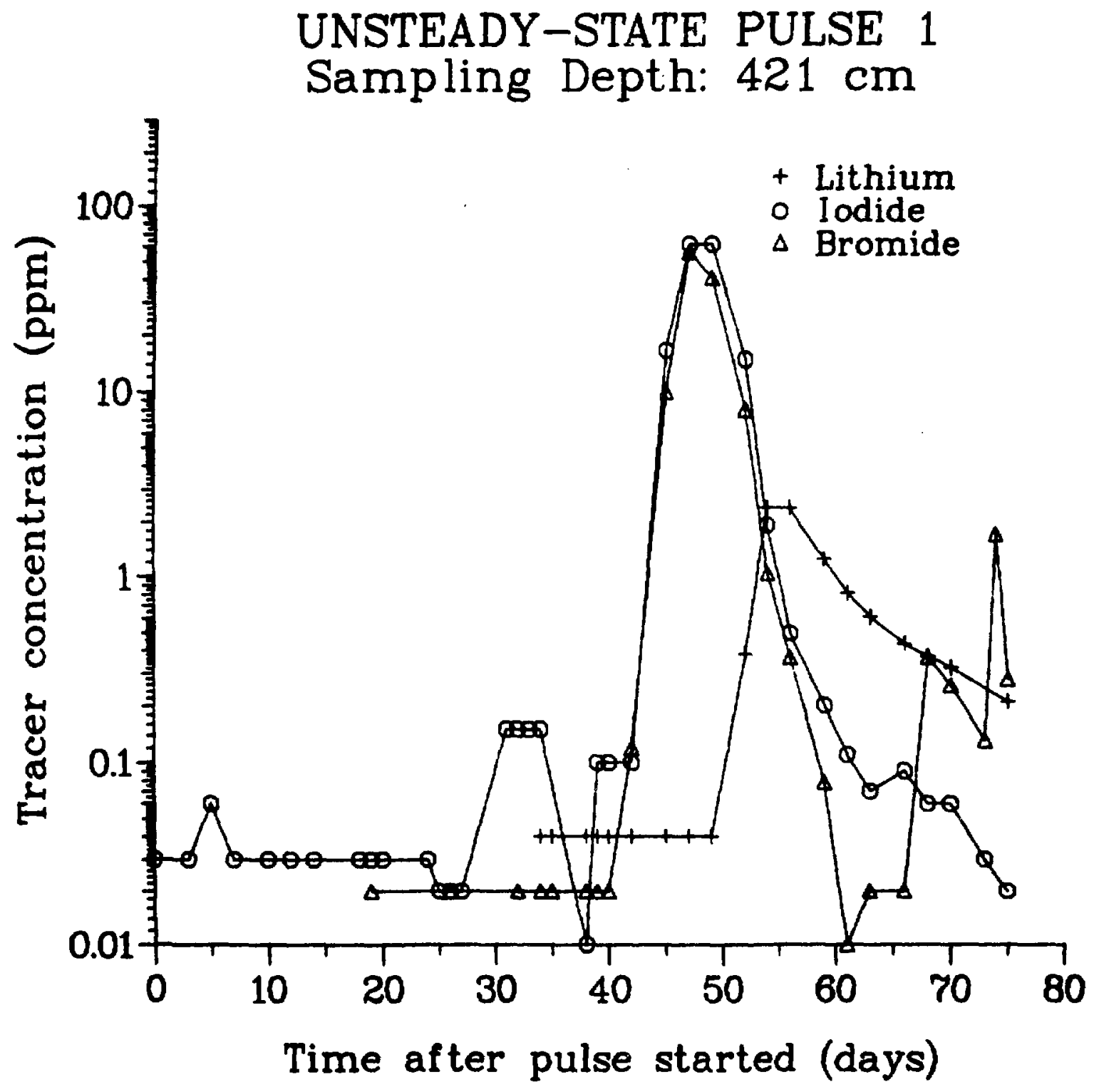

Fig. 10. Jodide, bromide, and lithium concentrations in Caisson B as a function of time after the unsteady-state pulse 1 was started at the $421-\mathrm{cm}$ depth. 


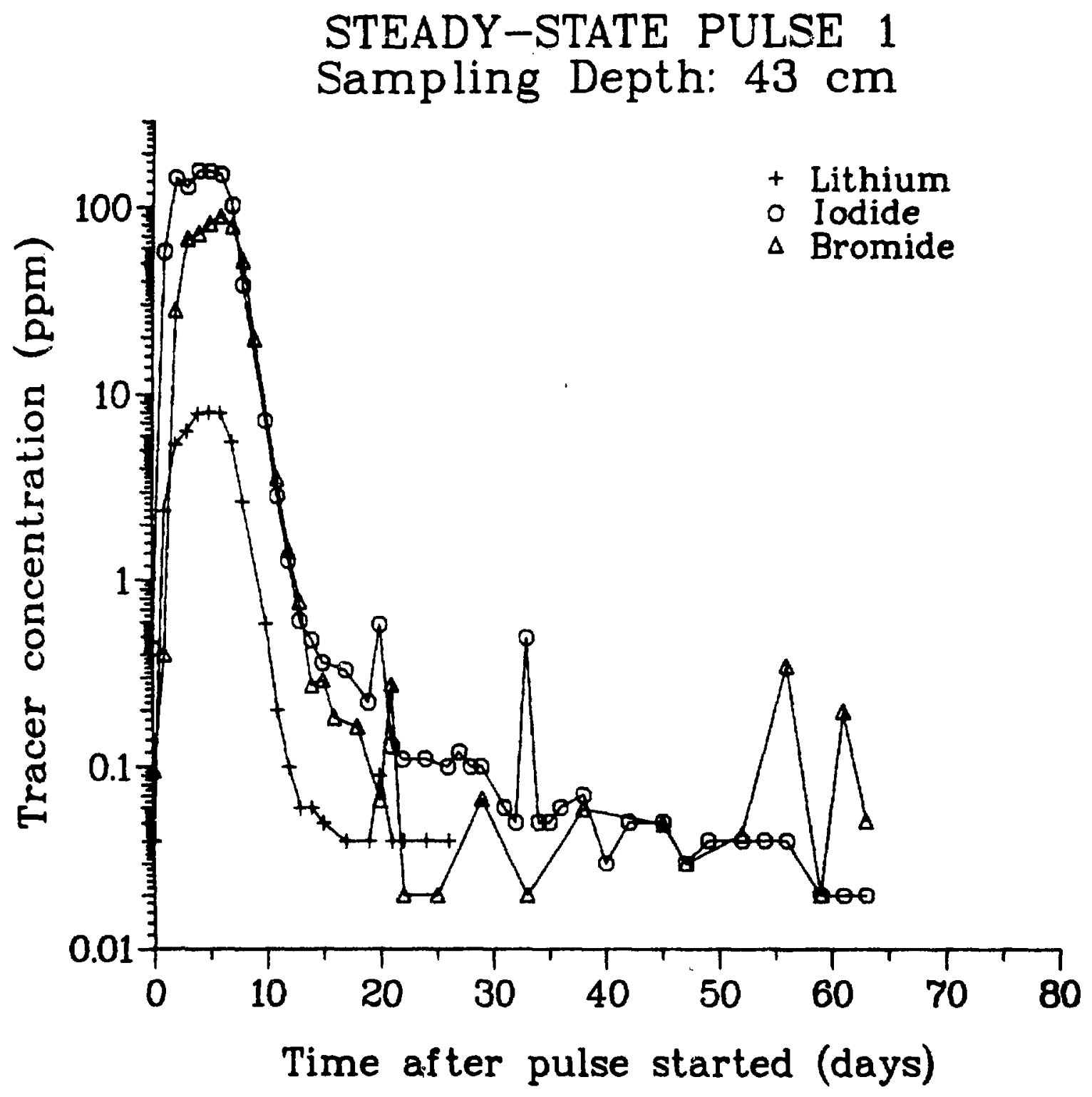

Fig. 11. Iodide, bromide, and lithium concentrations in Caisson B as a function of time after the steady-state pulse 1 was started at the 43-cm depth. 


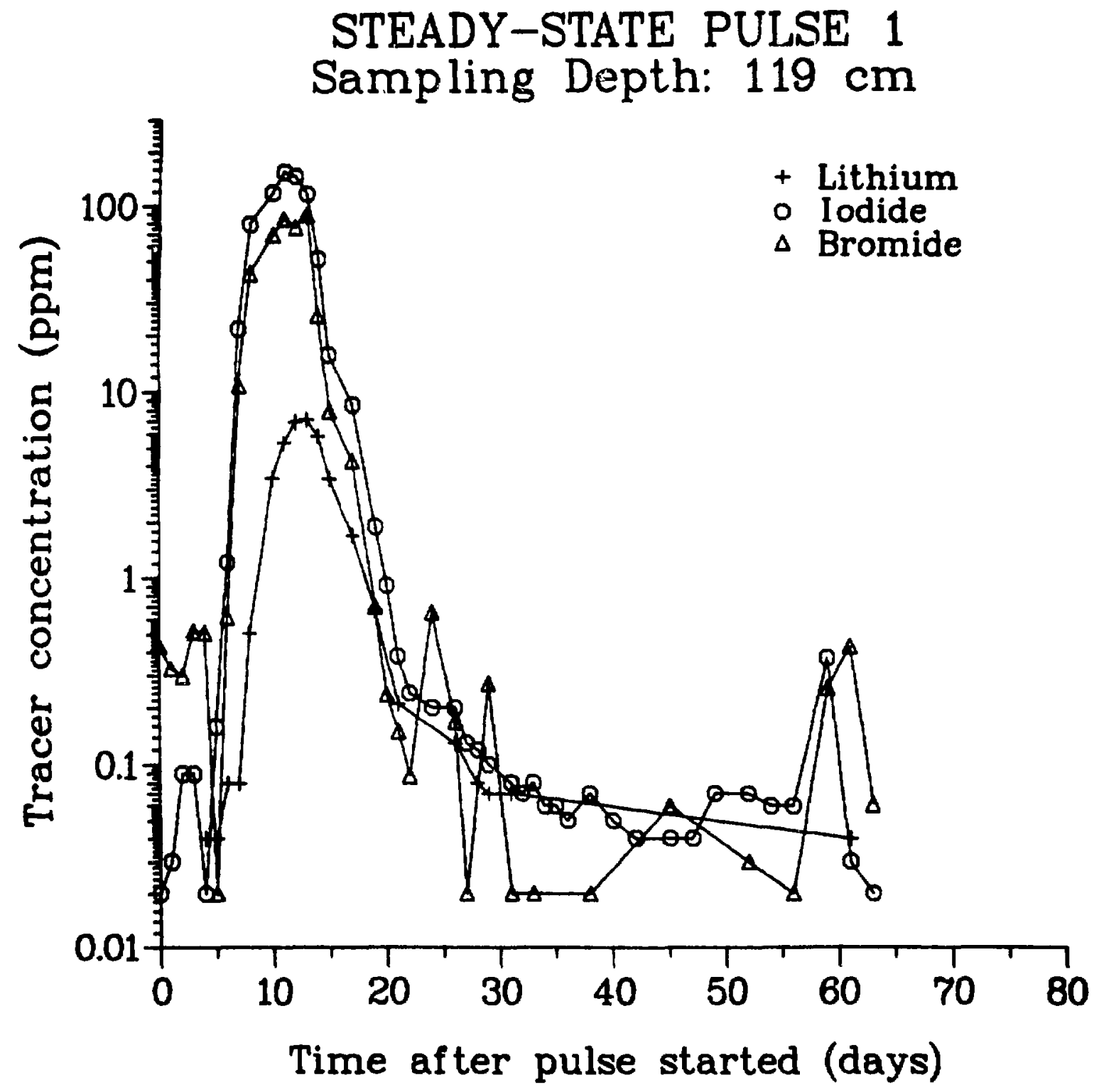

Fig. 12. Iodide, bromide, and lithium concentrations in Caisson B as a function of time after the steady-state pulse 1 was started at the $119-\mathrm{cm}$ depth. 


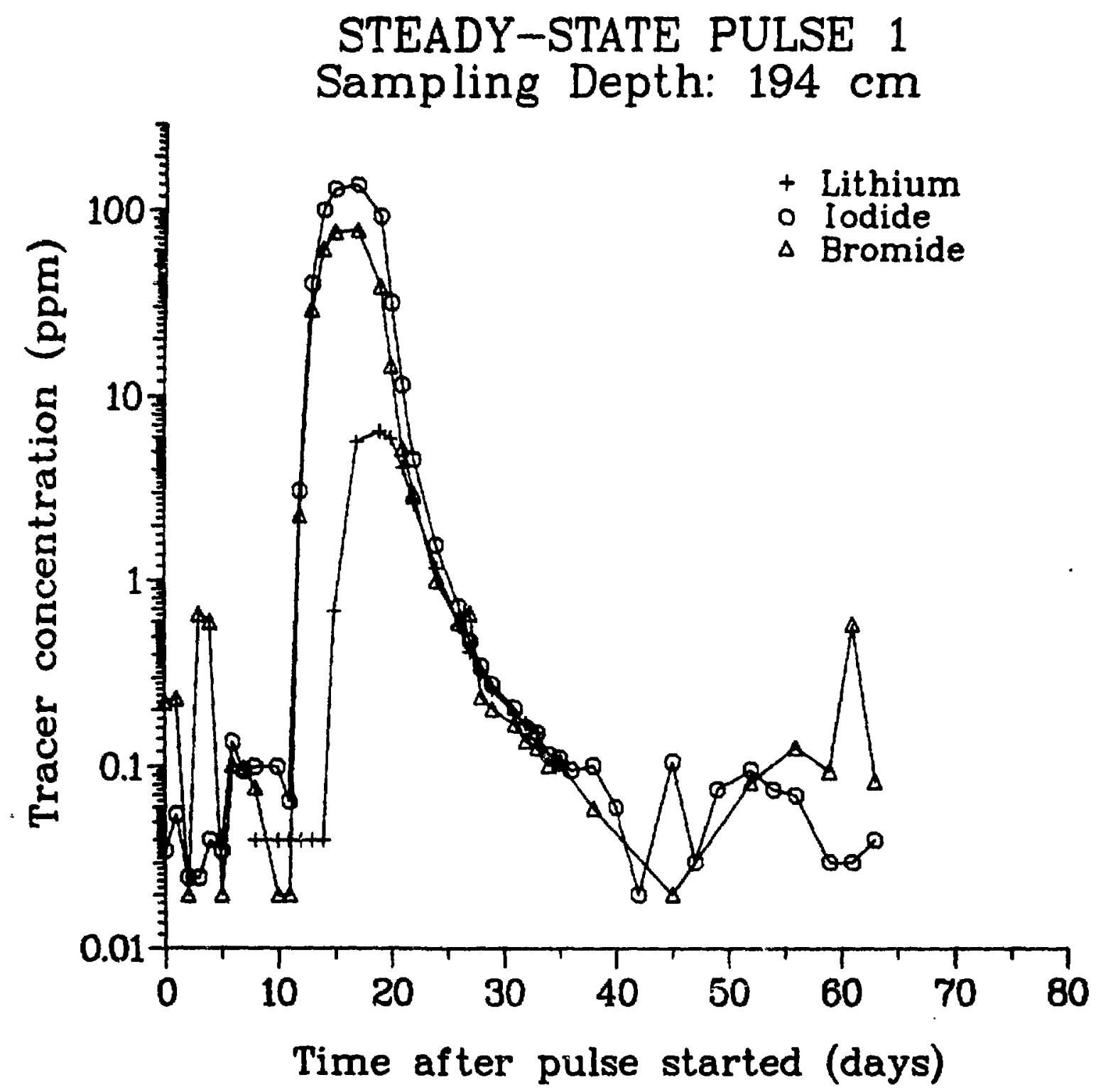

Fig. 13. Iodide, bromide, and lithium concentrations in Caisson B as a function of time after the steady-state pulse 1 was siarted at the 194-cm depth. 


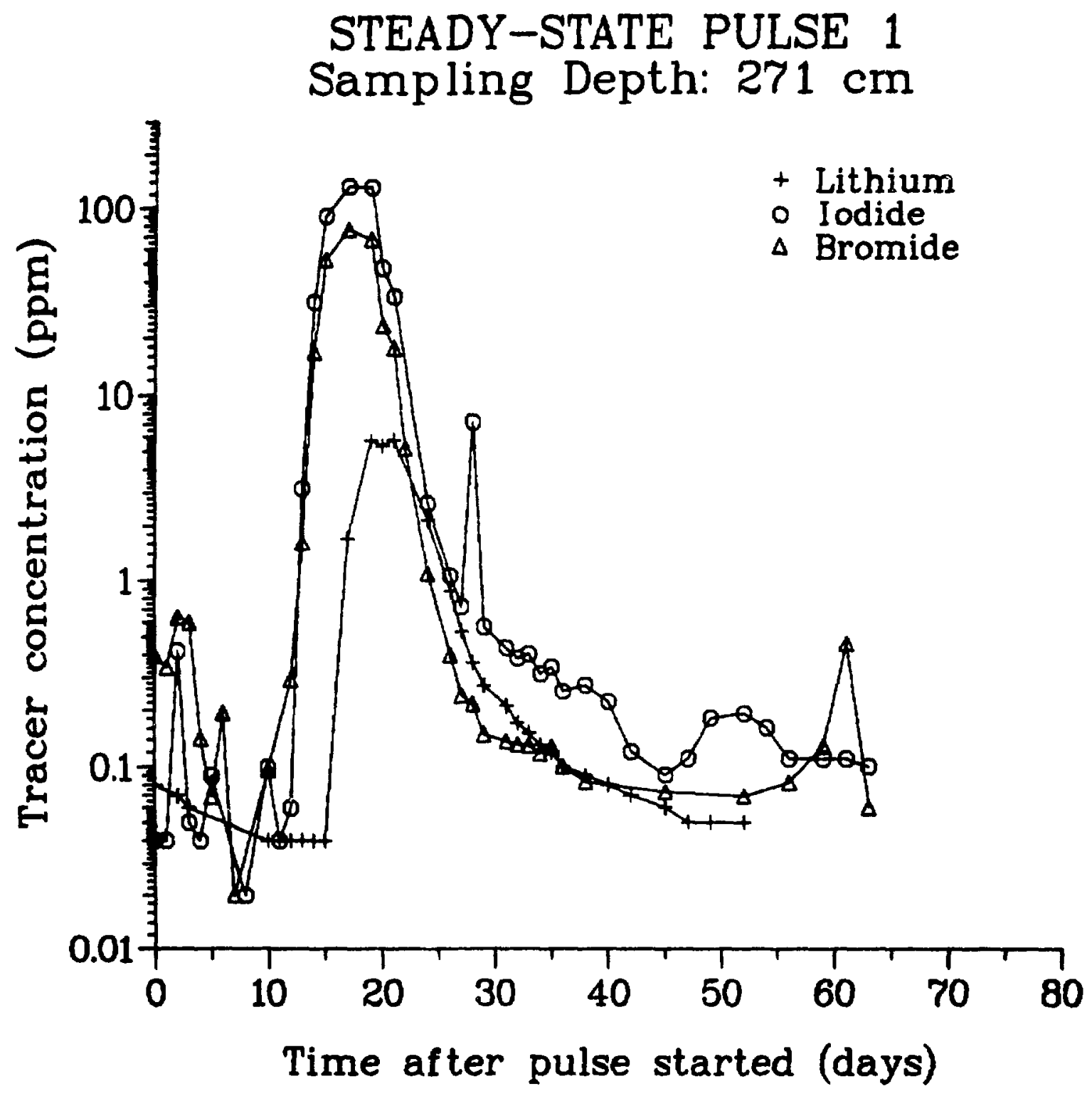

Fig. 14. Iodide, bromide, and lithium concentrations in Caisson B as a function of time after the steady-state pulse 1 was started at the $271-\mathrm{cm}$ depth. 


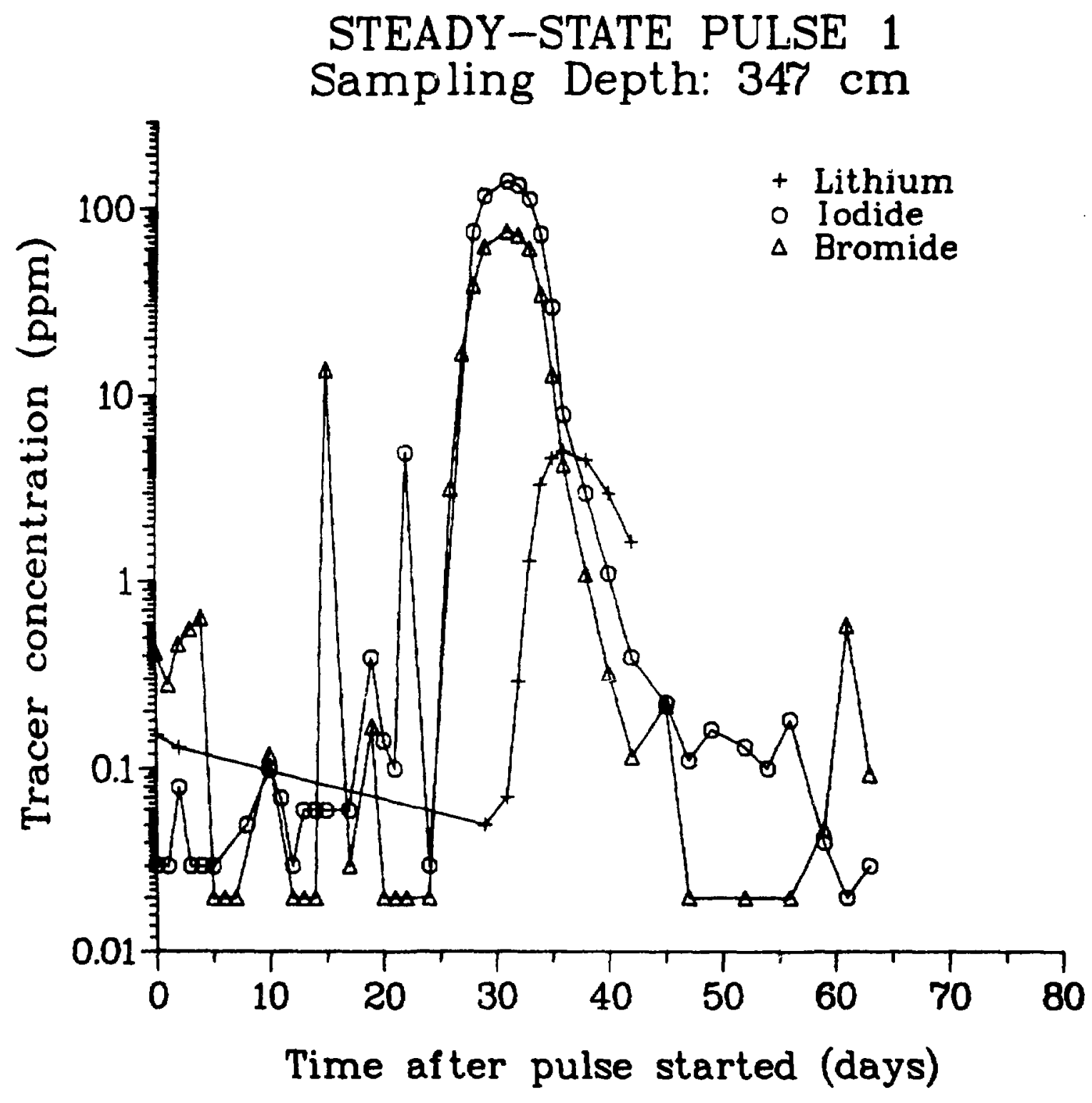

Fig. 15. Iodide, bromide, and lithium concentrations in Caisson B as a function of time after the steady-state pulse 1 was started at the $347-\mathrm{cm}$ depth. 


\section{STEADY-STATE PULSE 1 Sampling Depth: $421 \mathrm{~cm}$}

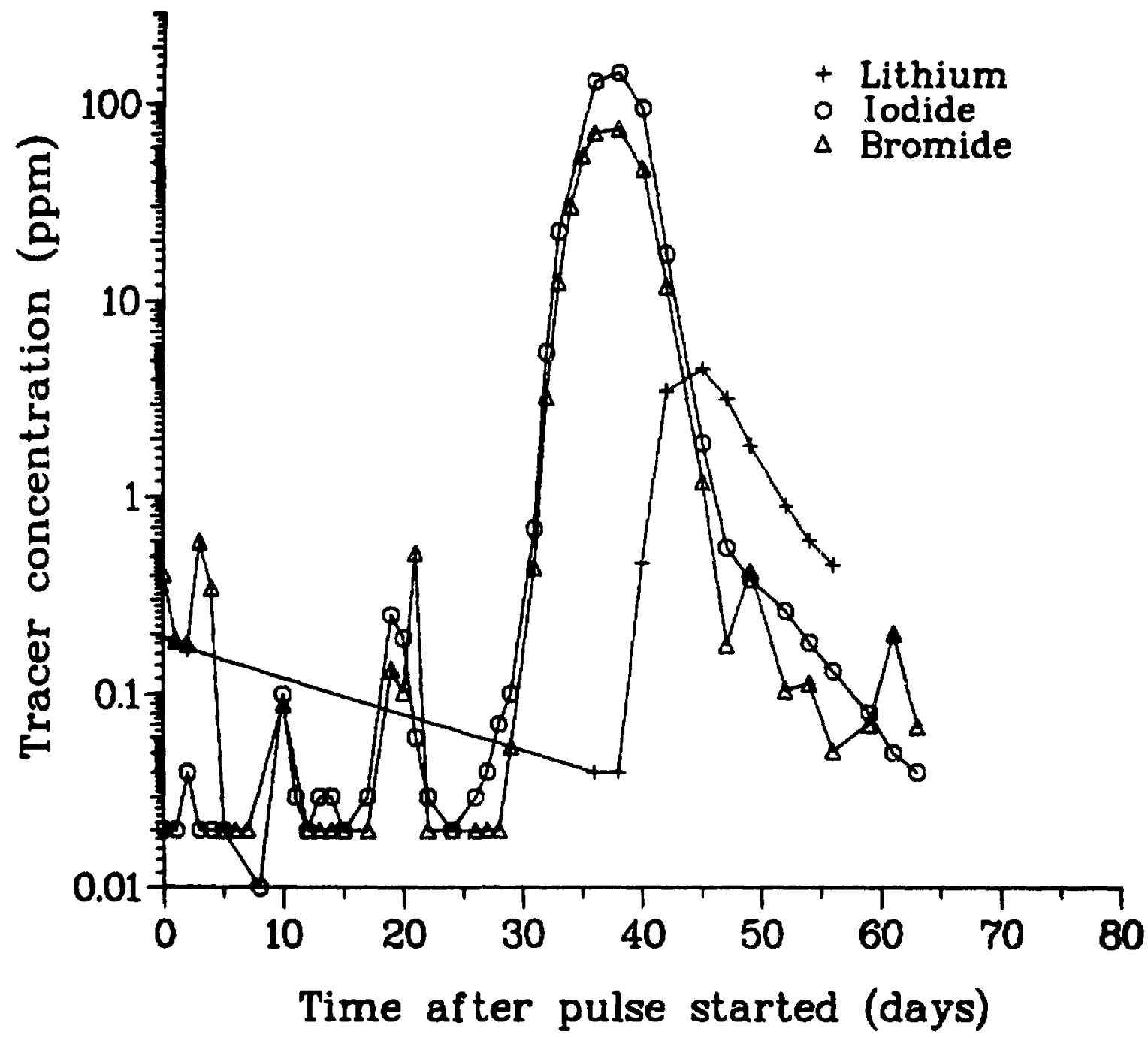

Fig. 16. Iodide, bromide, and lithium concentrations in Caisson B as a function of time after the steady-state pulse 1 was started at the $421-\mathrm{cm}$ depth. 


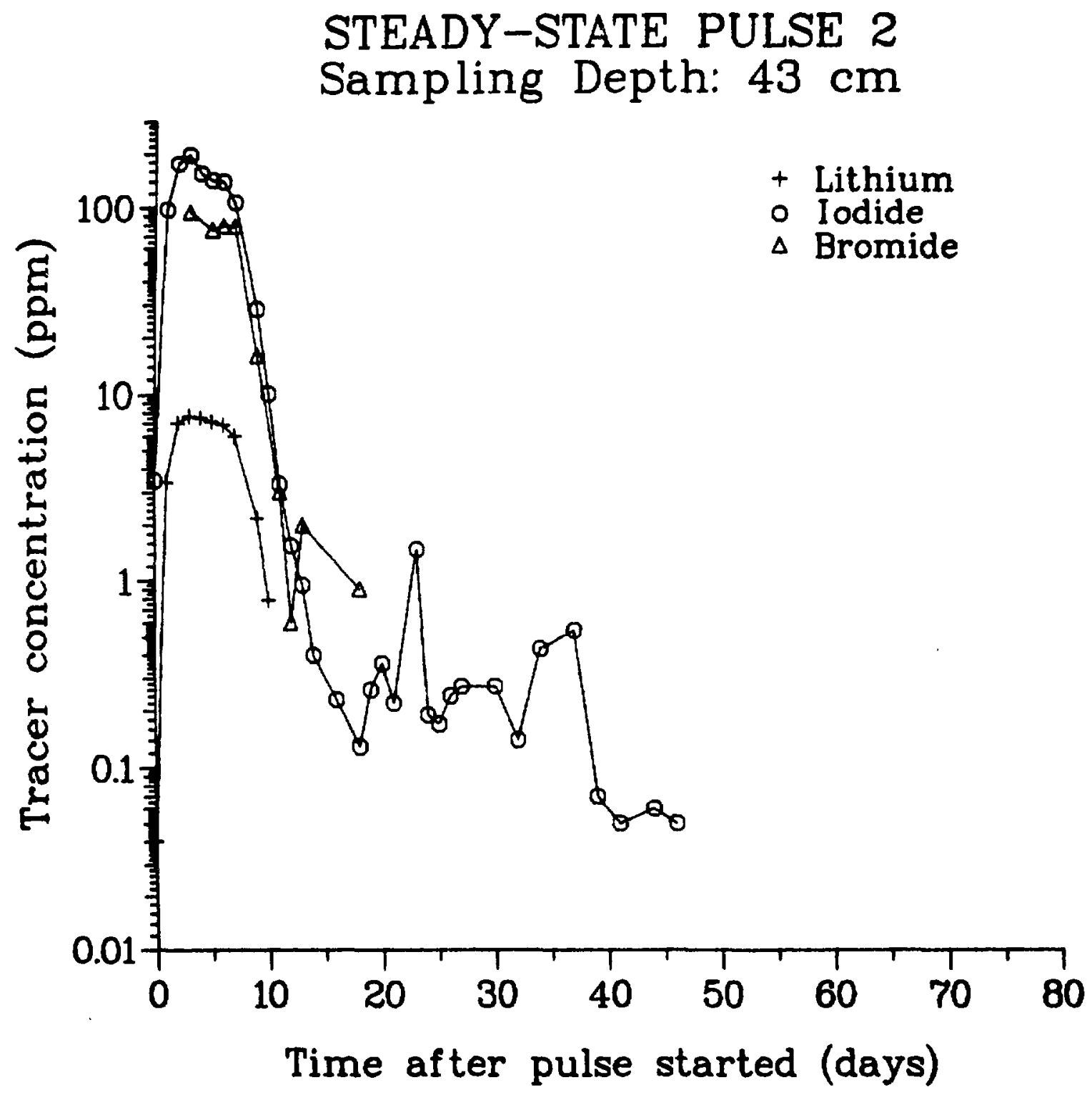

Fig. 17. Iodide, bromide, and lithium concentrations in Caisson B as a function of time after the steady-state pul se 2 was started at the $43-\mathrm{cm}$ depth. 
STEADY-STATE PULSE 2
Sampling Depth: $119 \mathrm{~cm}$

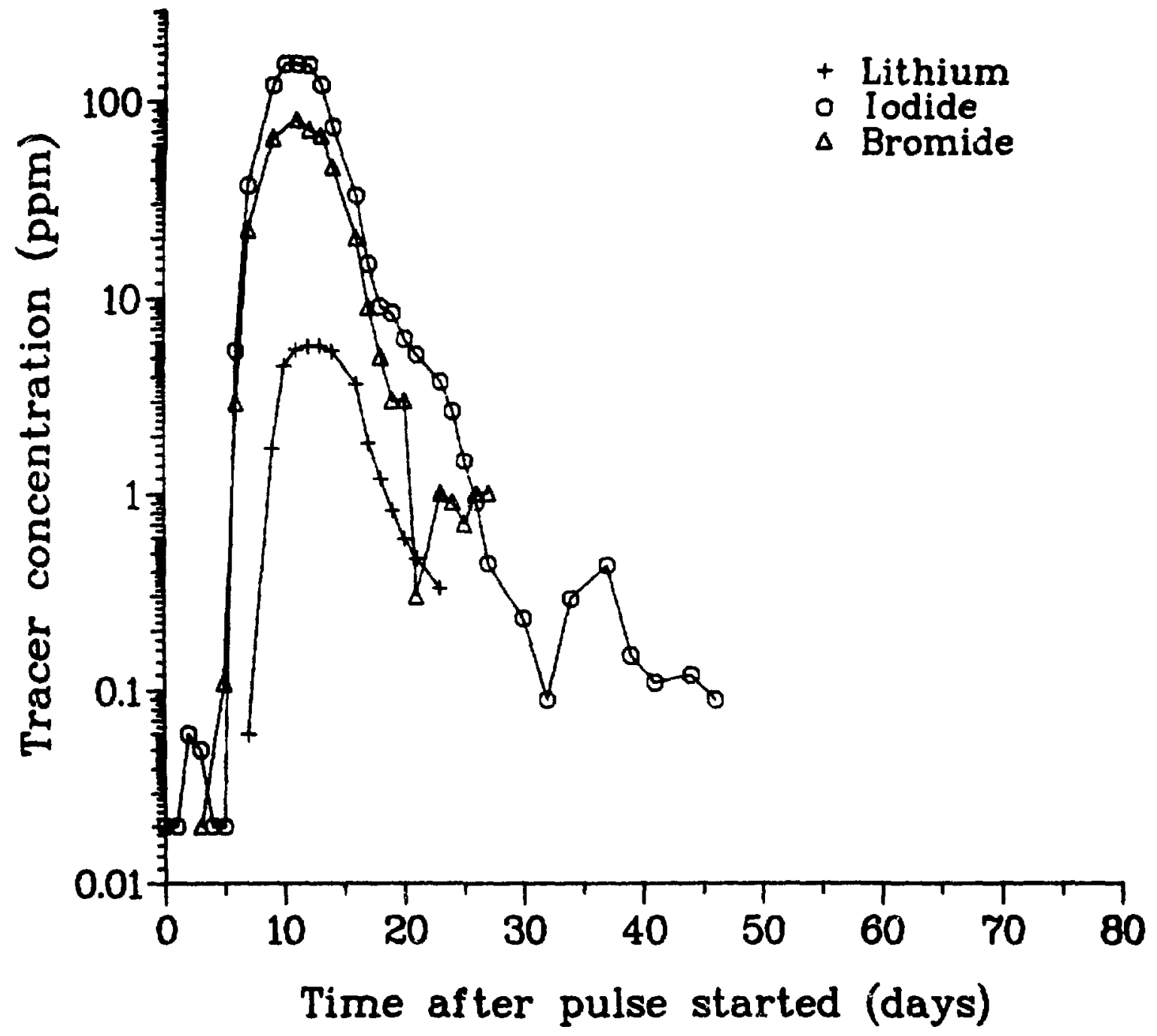

Fig. 18. Iodide, bromide, and lithium concentrations in Caisson B as a function of time after the steady-state pulse 2 was started at the 119-cm depth. 


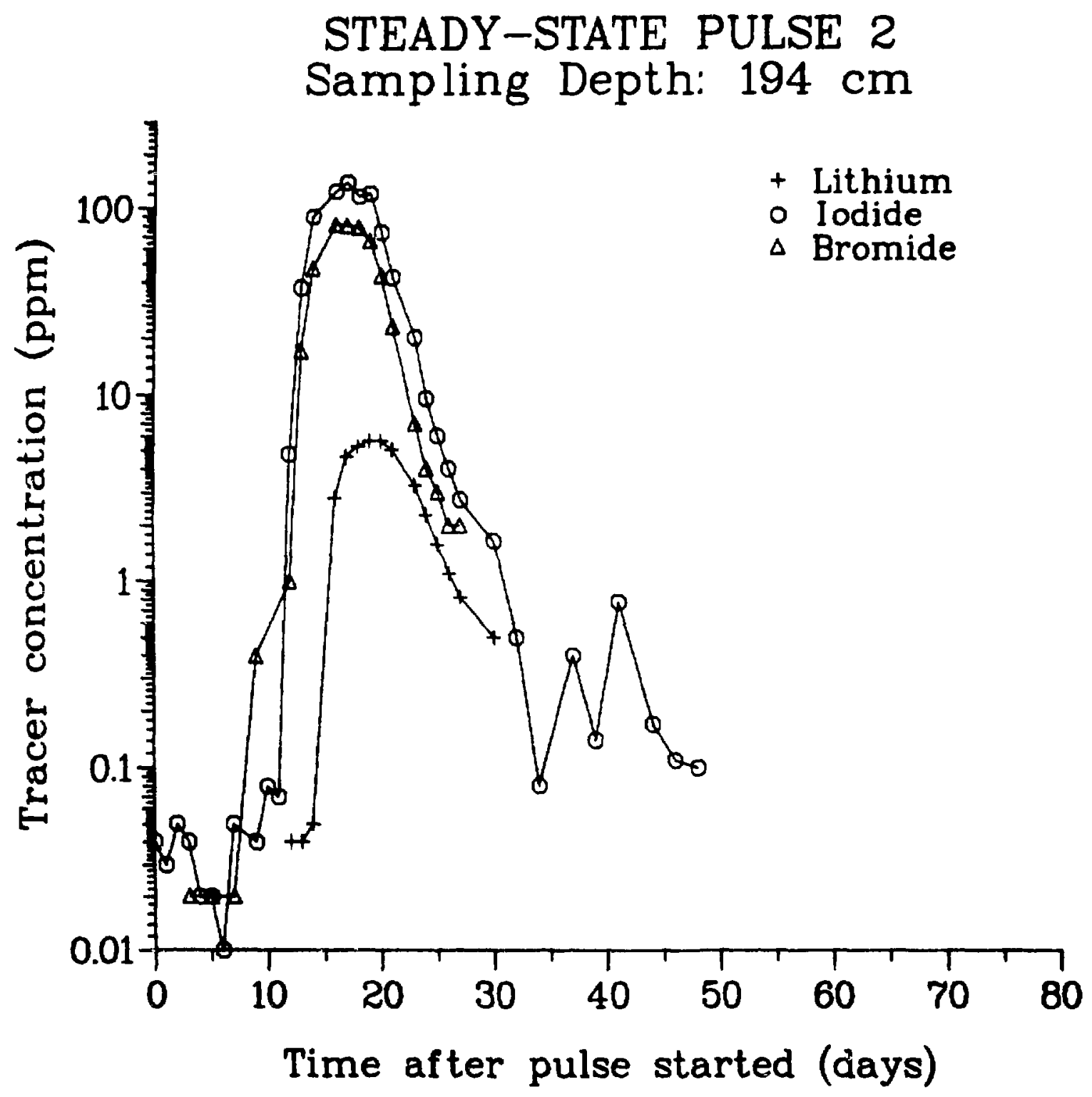

Fig. 19. Iodide, bromide, and lithium concentrations in Caisson B as a function of time after the steady-state pulse 2 was started at the 194-cm depth. 


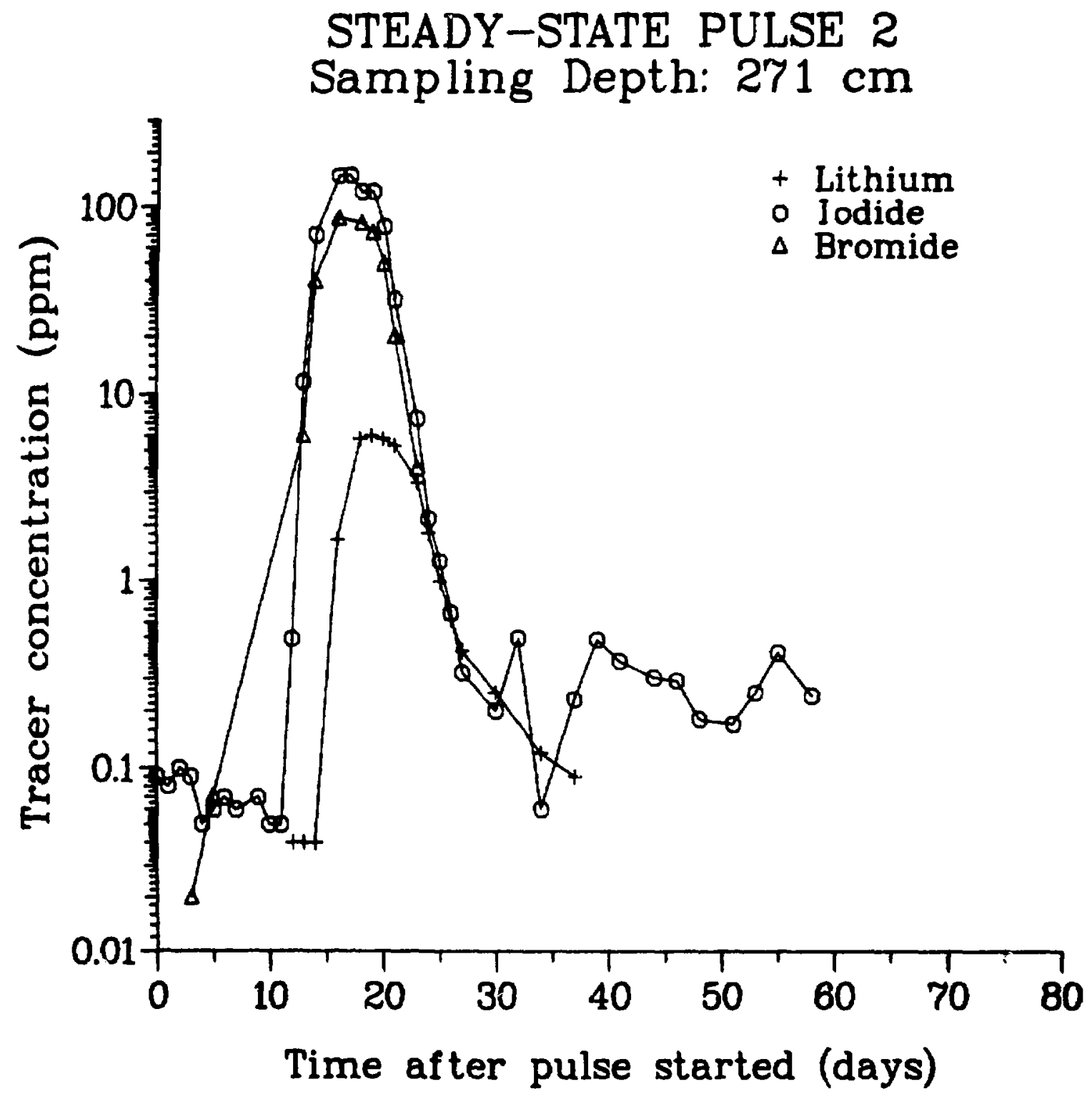

Fig. 20. Iodide, bromide, and lithium concentrations in Caisson B as a function of time after the steady-state pulse 2 was started at the $271-\mathrm{cm}$ depth. 


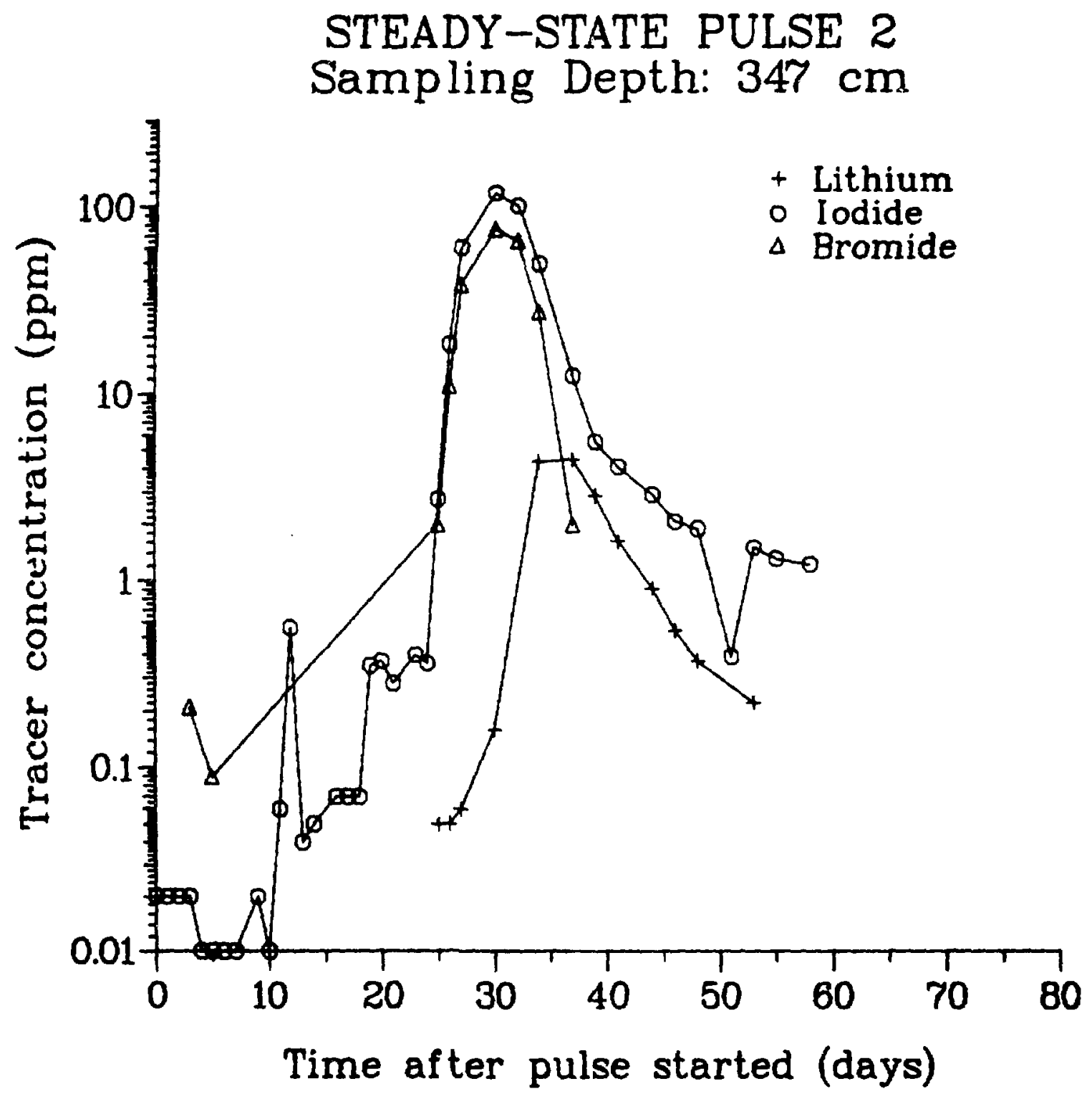

Fig. 21. Iodide, bromide, and Iithium concentrations in Caisson B as a function of time after the steady-state pulse 2 was started at the $347-\mathrm{cm}$ depth. 


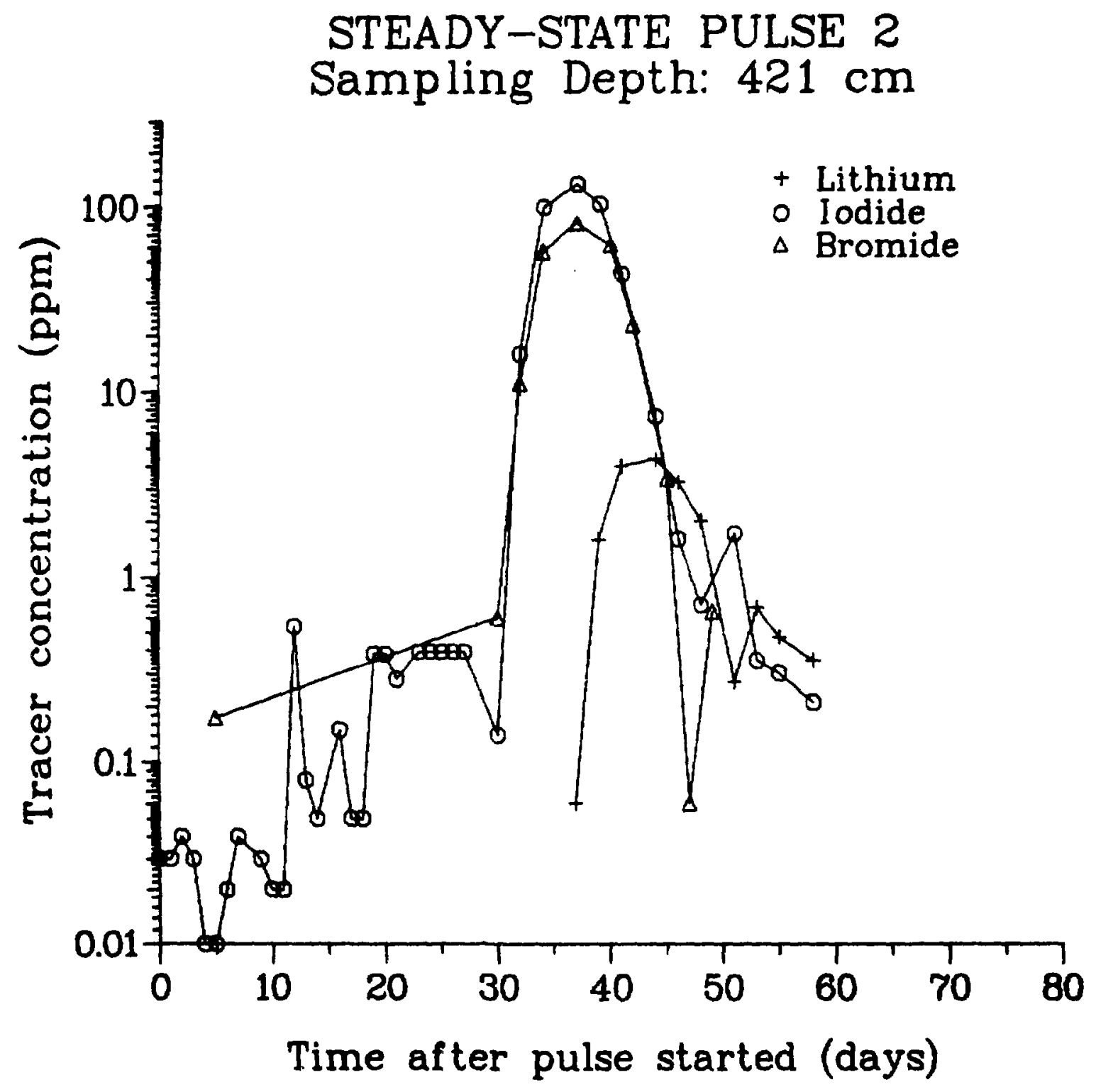

Fig. 22. Iodide, bromide, and lithium concentrations in Caisson B as a function of time after the steady-state pulse 2 was started at the $421-\mathrm{cm}$ depth. 


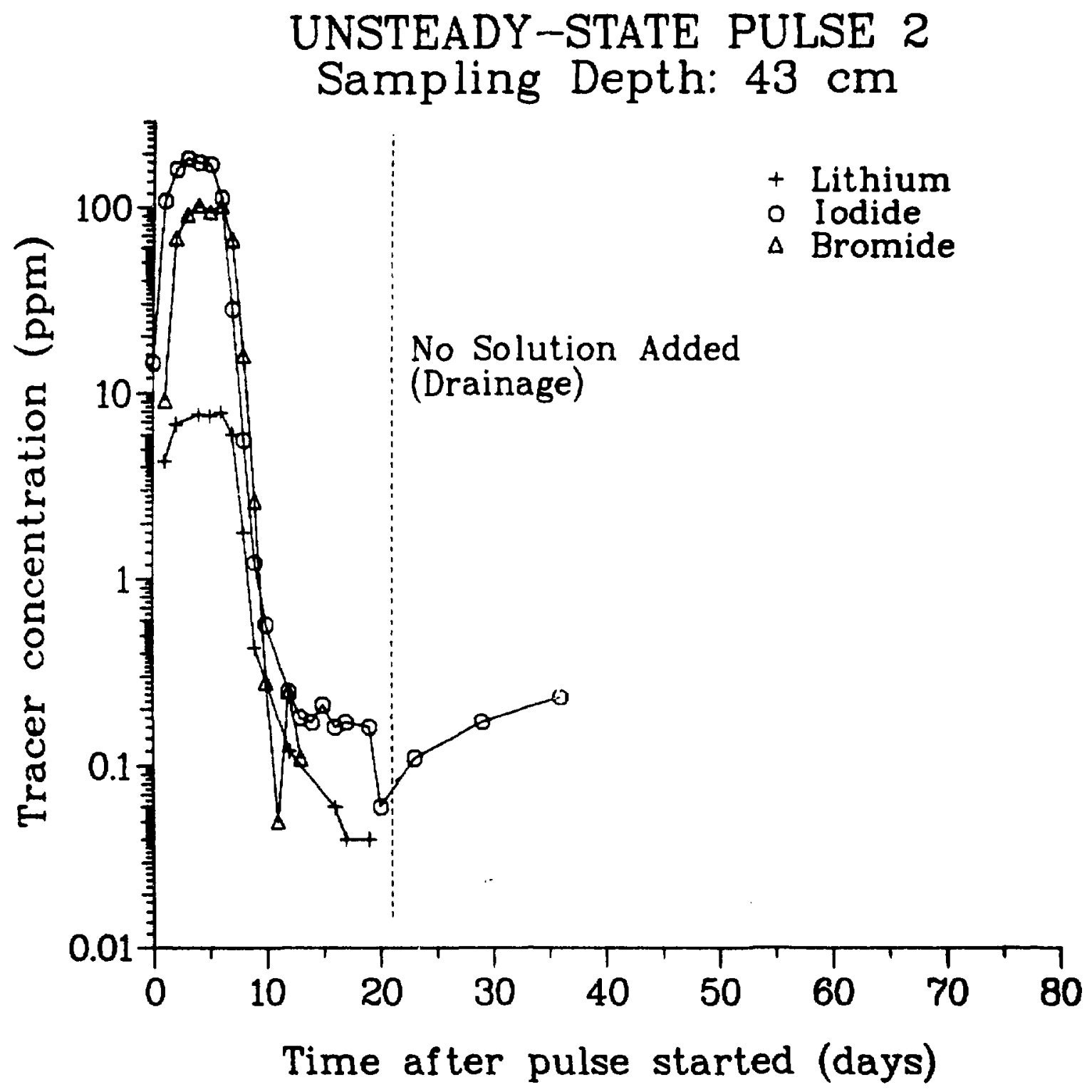

Fig. 23. Iodide, bromide, and 7 ithium concentrations in Caisson B as a function of time after the unsteady-state pulse 2 was started at the $43-\mathrm{cm}$ depth. 


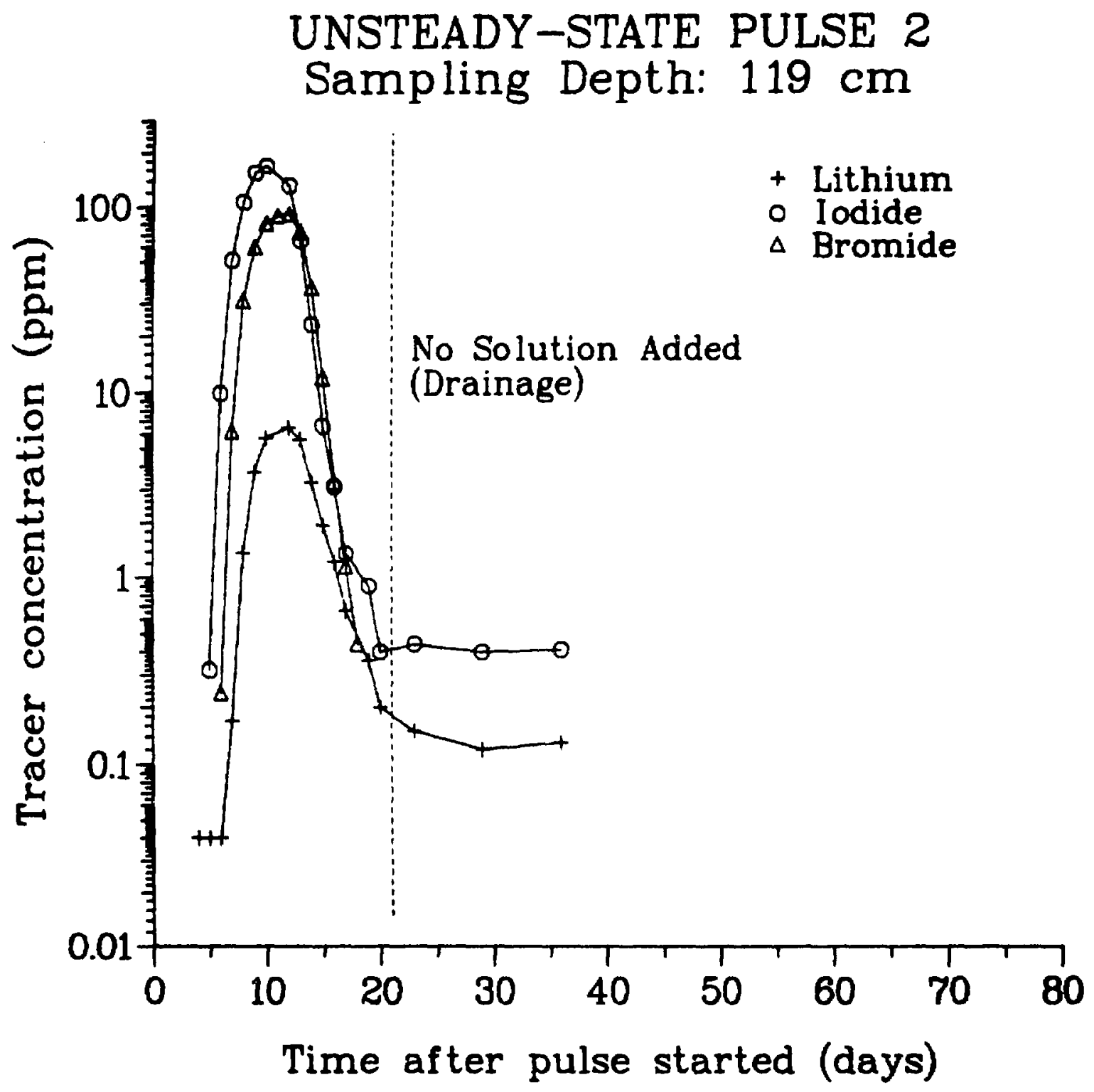

Fig. 24. Iodide, bromide, and lithium concentrations in Caisson B as a function of time after the unsteady-state pulse 2 was started at the $119-\mathrm{cm}$ depth. 


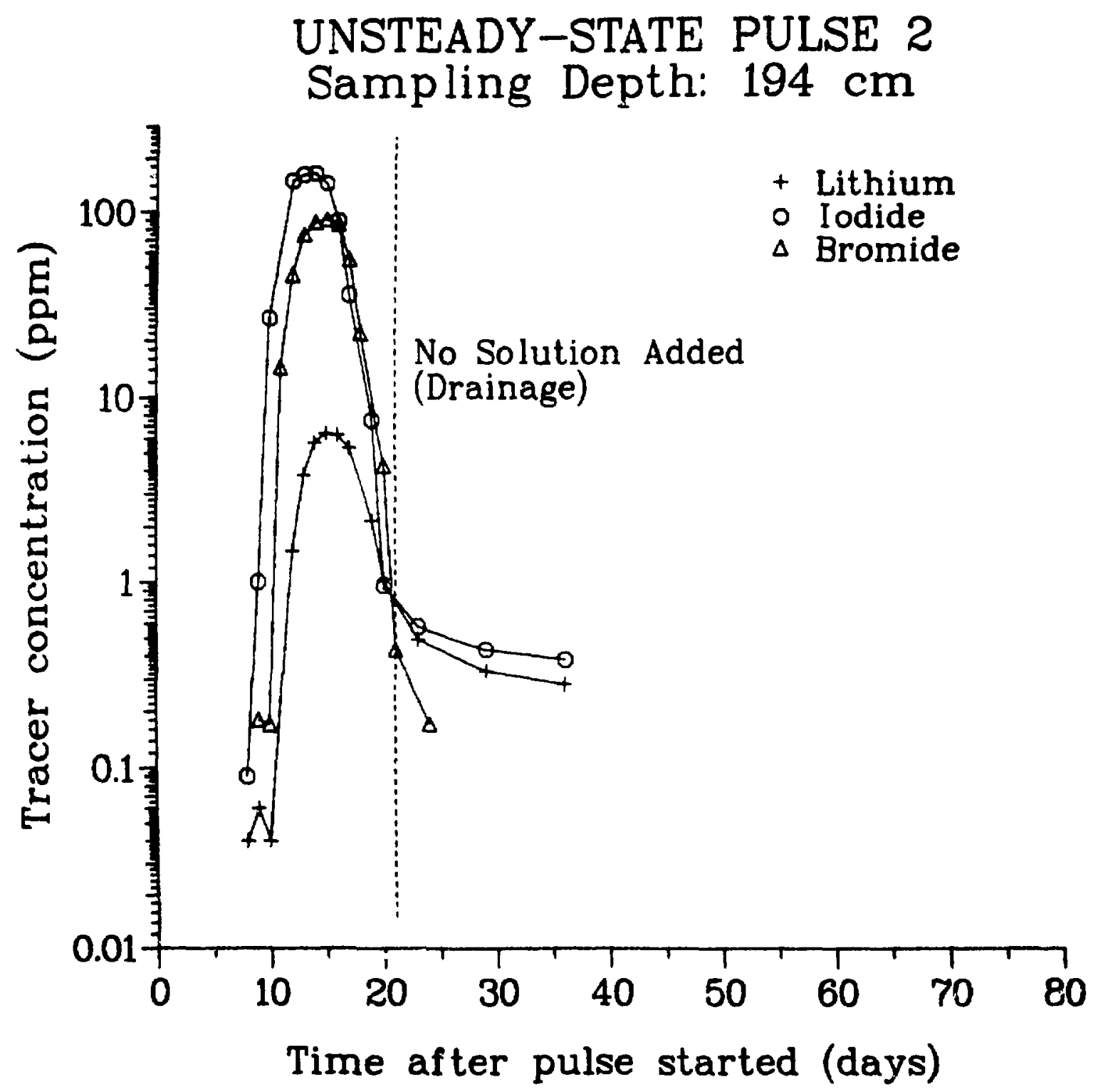

Fig. 25. Iodide, bromide, and lithium concentrations in Caisson B as a function of time after the unsteady-stace pulse 2 was started at the 194-cm depth. 


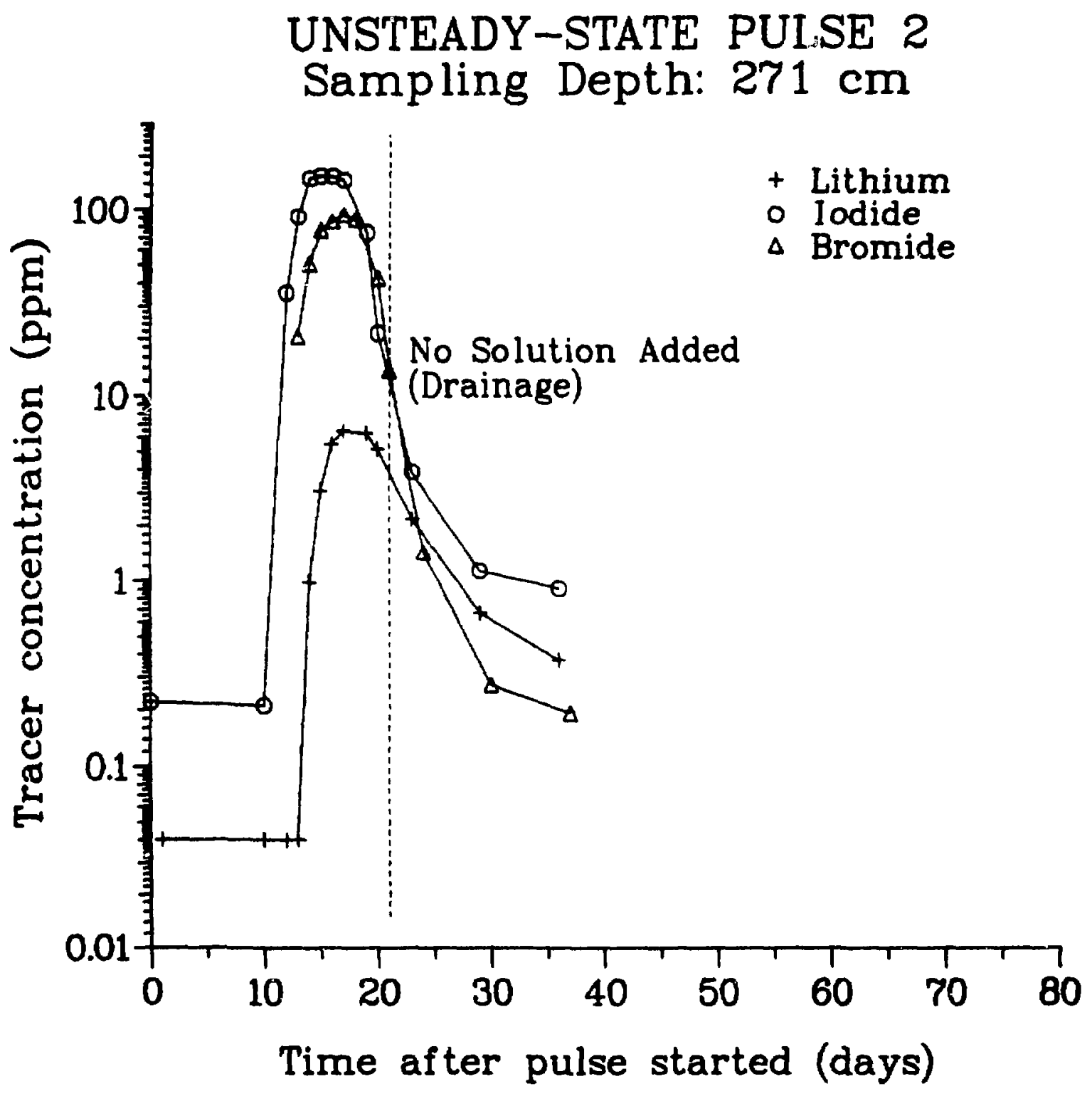

Fig. 26. Iodide, bromide, and lithium concentrations in Caisson B as a function of time after the unsteady-state pulse 2 was started at the $271-\mathrm{cm}$ depth. 


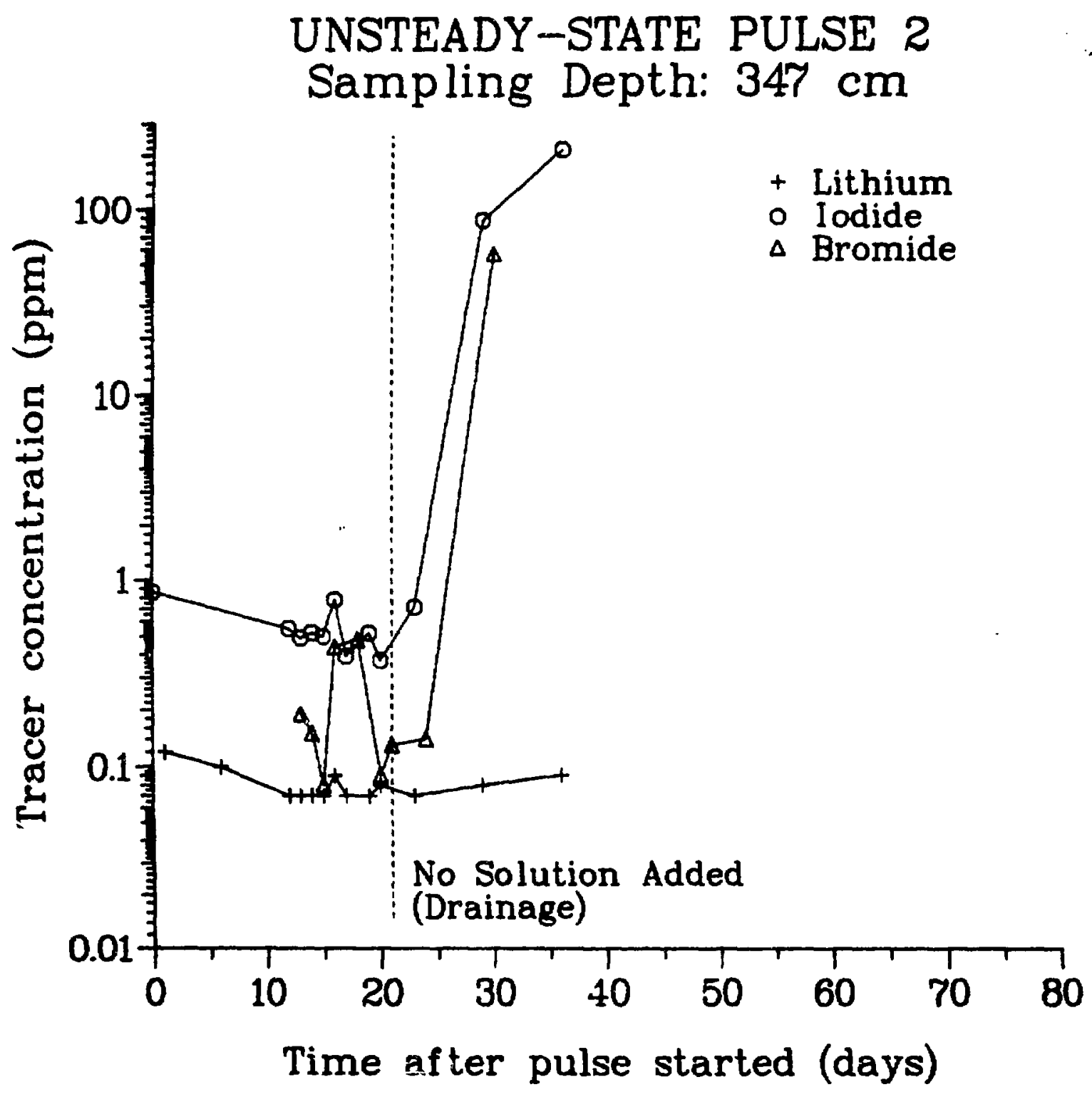

Fig. 27. Iodide, bromide, and lithium concentrations in Caisson B as a function of time after the unsteady-state pulse 2 was started at the $347-\mathrm{cm}$ depth. 


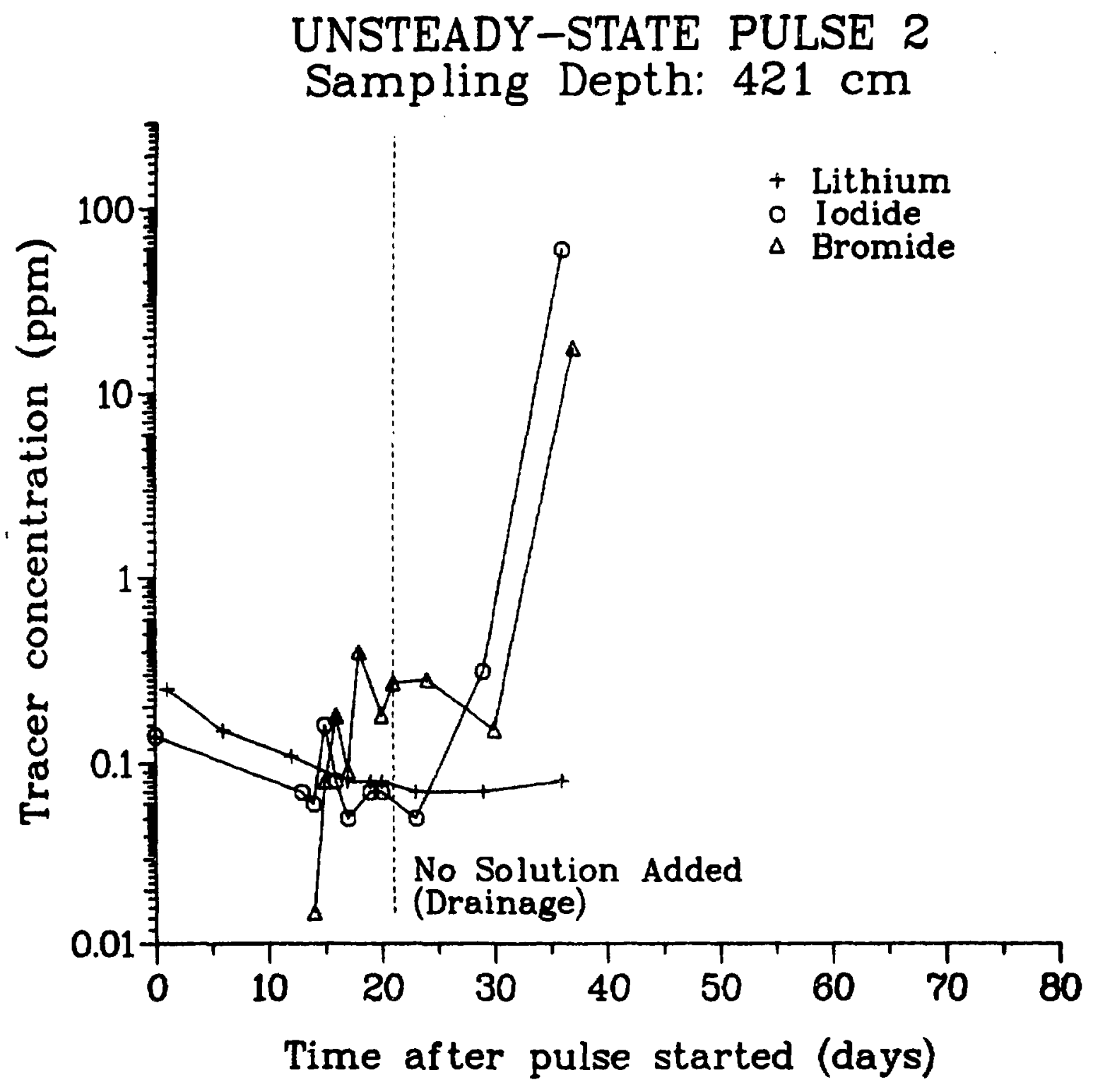

Fig. 28. Iodide, bromide, and lithium concentrations in Caisson o as a function of time after the unsteady-state pulse 2 was started at the 421-cm depth. 


\section{lodide migration: first steady-state pulse}

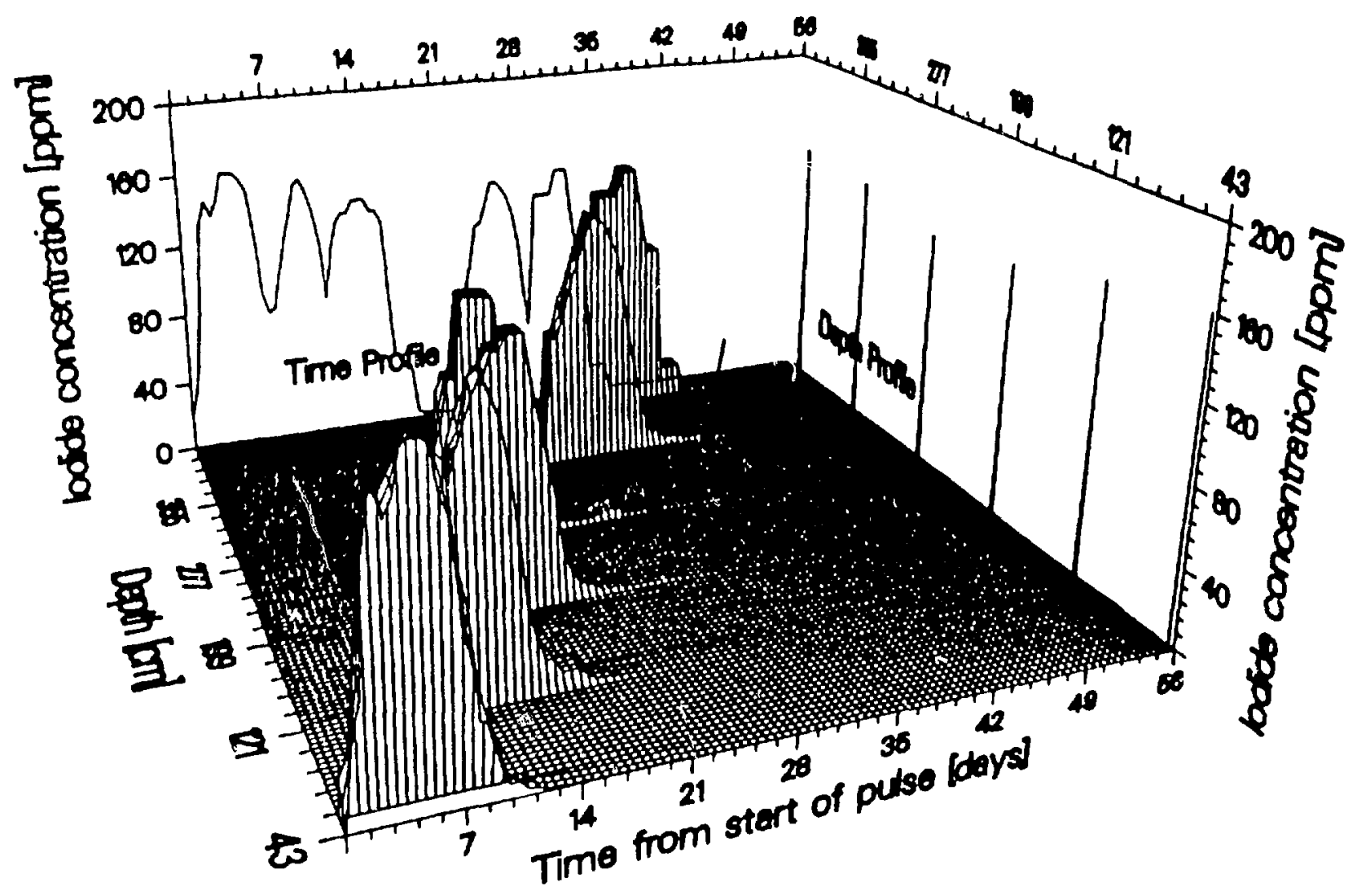

Fig. 29. Iodide concentration in the iirst steady-state pulse experiment in Caisson B as functions of depth and time. 


\section{lodide migration: second steady-state pulse}

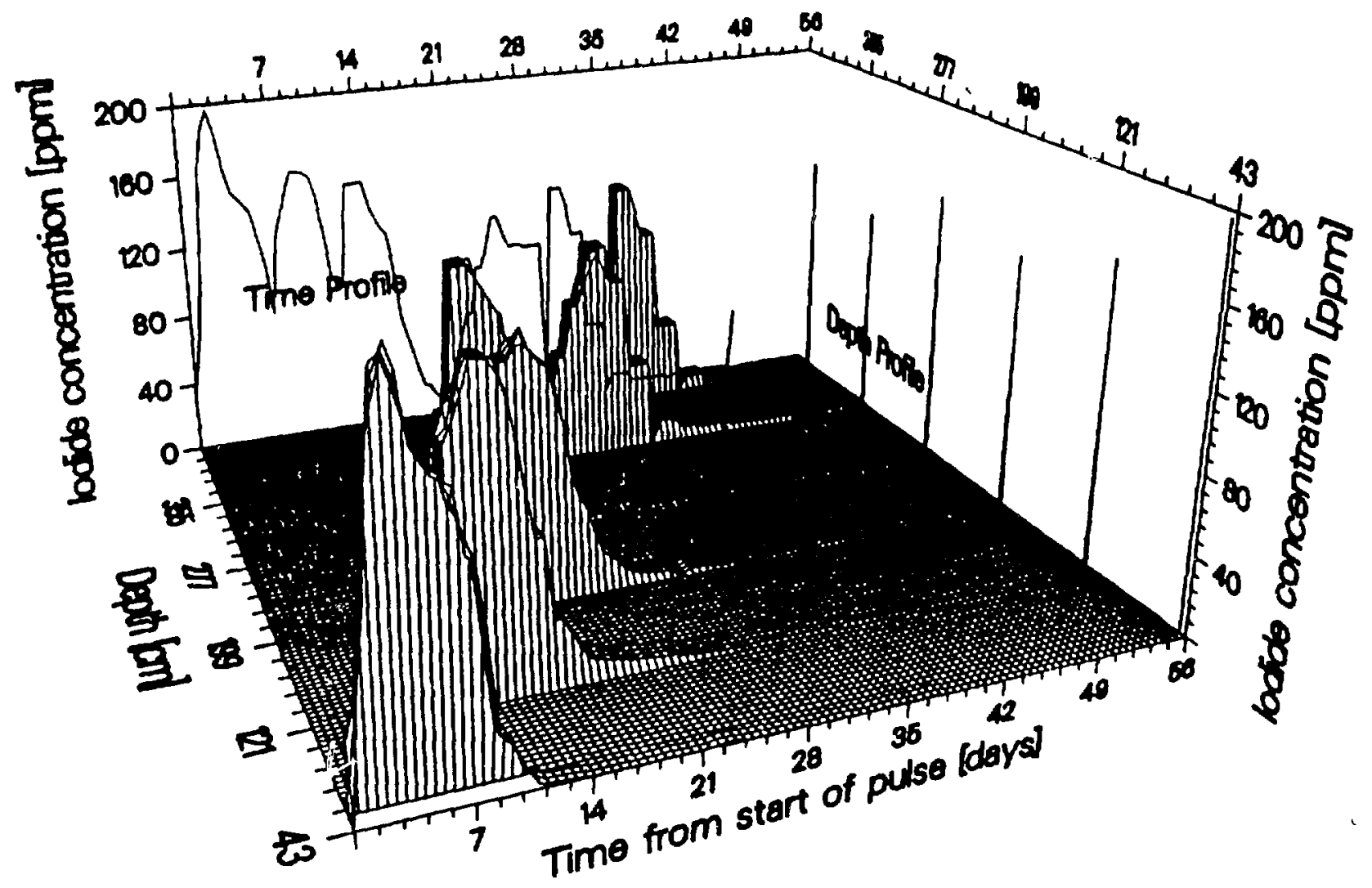

Fig. 30. Iodide concentration in the second steady-state pulse experiment in Caisson $B$ as functions of depth and time. 


\section{lodide migration: first unsteady-state pulse}

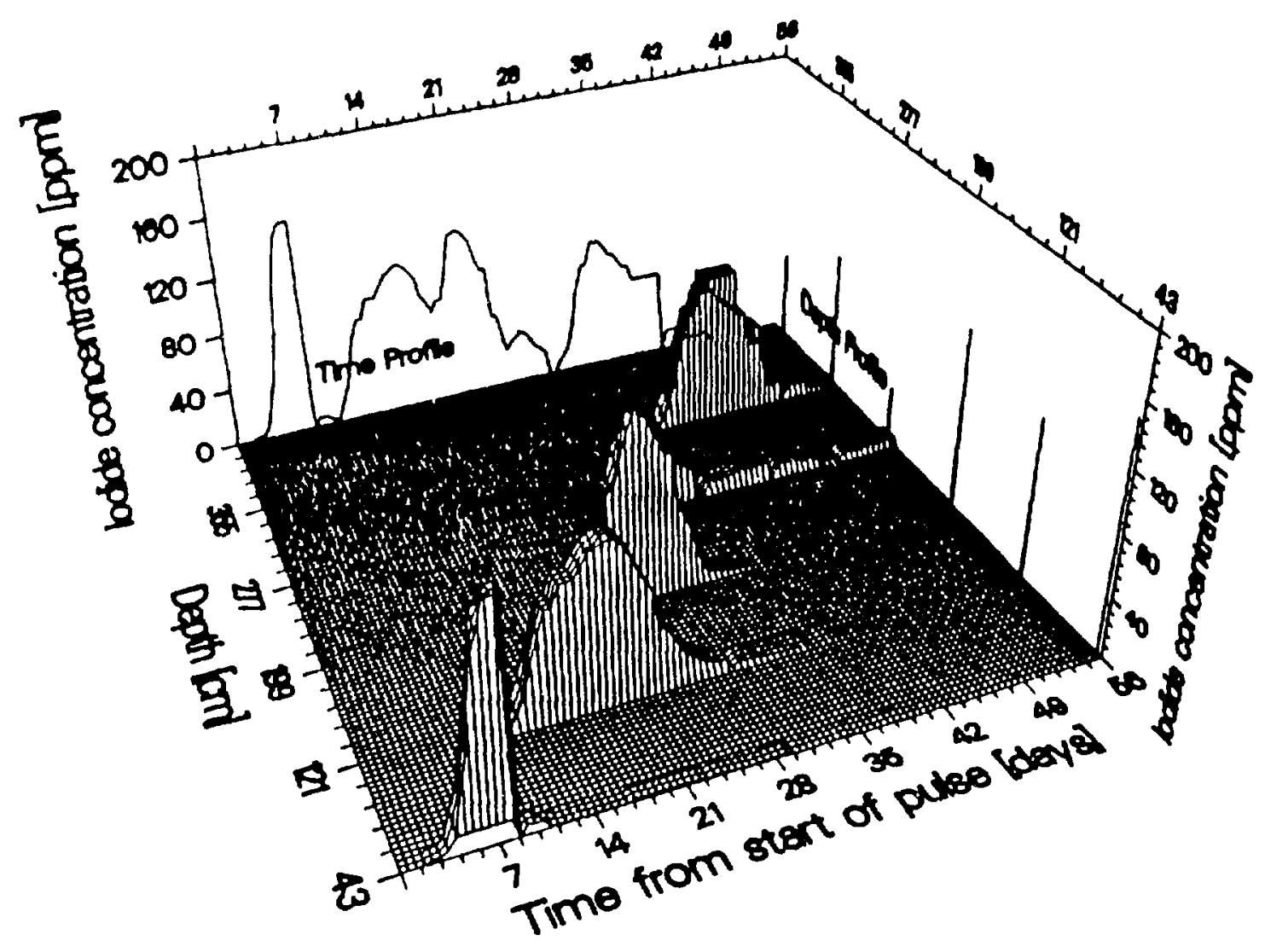

Fig. 31. Iodide concentration in the first unsteady-state pulse experiment in Caisson B as functions of depth and time. 


\section{lodide migration: second unsteady-state pulse}

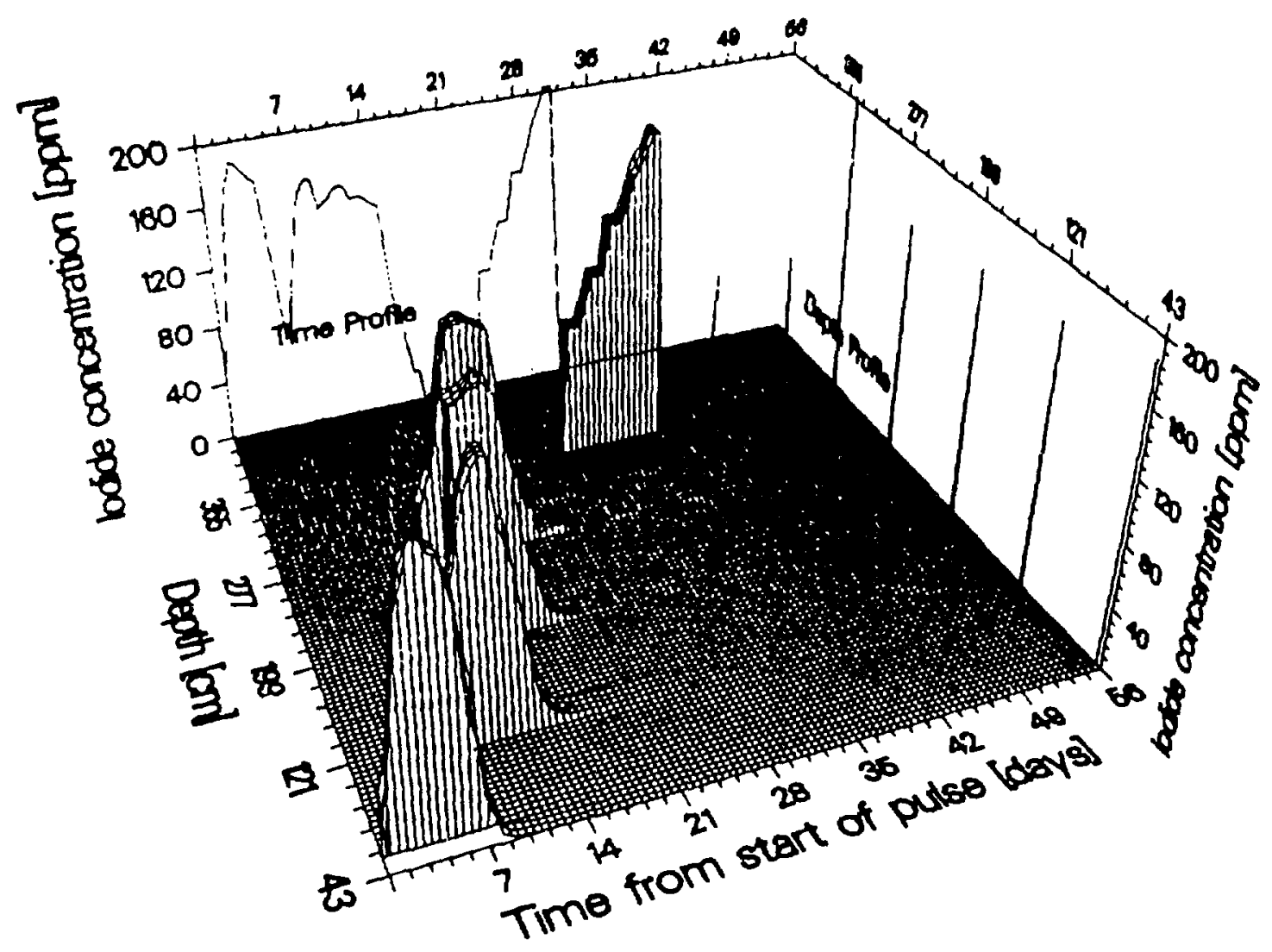

Fig. 32. Jodide concentration in the first second unsteady-state pulse experiment in Caisson $B$ as functions of depth and $t$ ime. 

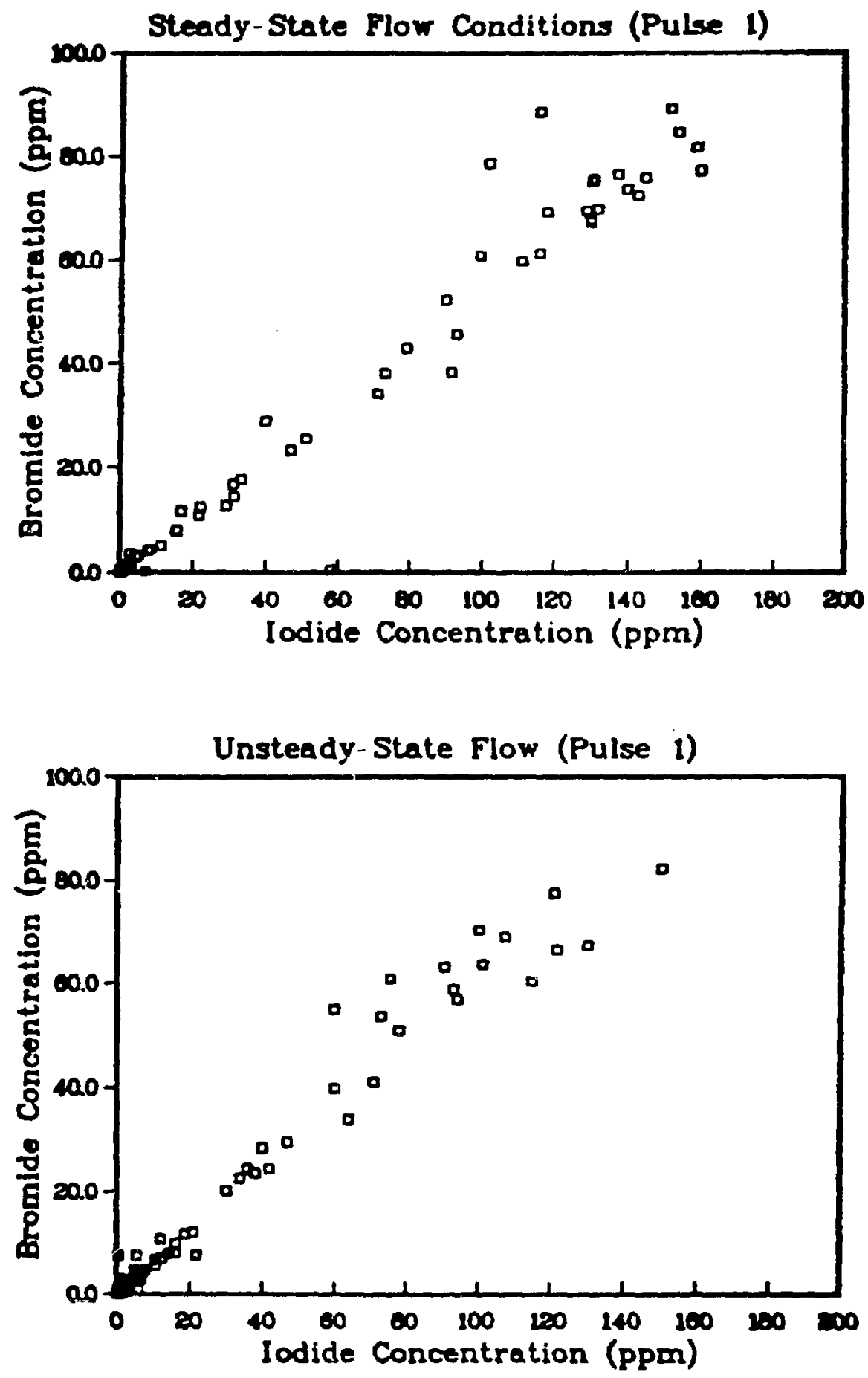

Fig. 33. Iodide versus bromide concentrations for two pulses in the Caisson $B$ experiment. 


\section{Lithium migration: first steady-state pulse}

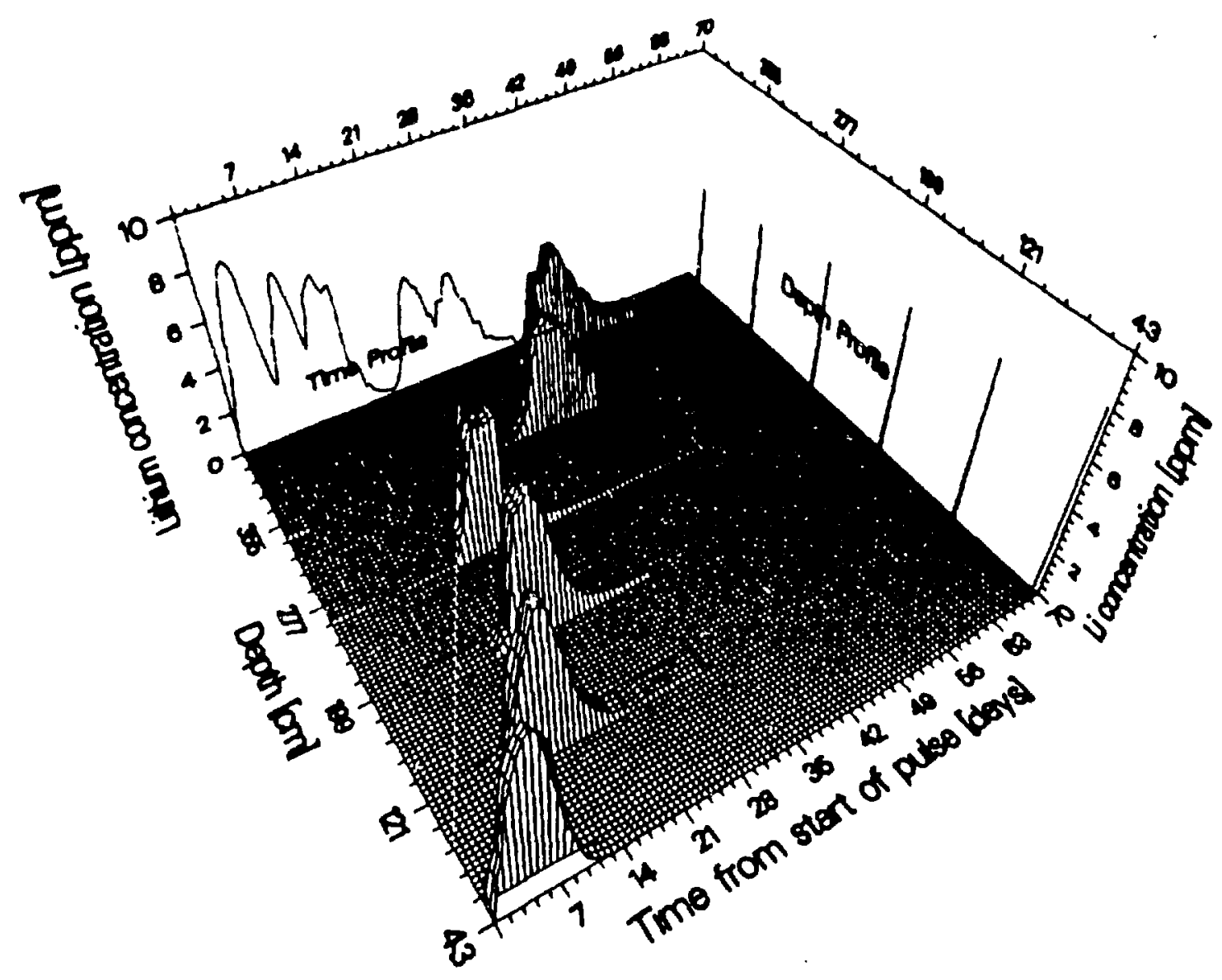

Fig. 34. Lithium concentrations in the first steady-state pulse experiment in Caisson $B$ as functions of depth and time. 
B. Tracer Data from Vertical Samplers

Using the vertical samplers, we were able to study the transport of tracers in the upper $118 \mathrm{~cm}$ of Caisson $B$ in more detail than with the samplers located at the access ports in the top $421 \mathrm{~cm}$ of the caisson. Although these samplers will probably be the only samplers used for cesium transport (cesium was observed to be very strongly absorbed by the tuff in the upper portions of the caisson), they will be used to generate a second set of absorption parameter estimates for iodide, lithium, and bromide in future data analysis. Basically, only steady-state flow conditions were studied for iodide, bromide, and lithium in these samplers (Figs. 35-46). All of the conclusions drawn for the transport of these three tracers, using the samplers located at the access ports, also apply to this data set (see previous section). Included in this set of conclusions, in spite of the short travel distance ( 43 $\mathrm{cm}$ to $188 \mathrm{~cm}$ ), was the observation that lithium migrated slower than iodide and bromide through the crushed tuff profile. C. Modeling Efforts

The following information covers only the results from modeling efforts for iodide, bromide, and lithium transport in the pulses of August 1983, September 1984, and December 1984. Modeling consisted of estimating the dispersion coefficient and retardation factor by an optimal parameter curve-fitting applied to an analytical solution of the advective-dispersive equation. The August 1983 and September 1984 pulses occurred at unsteadystate flow. Flow through the caisson was maintained at about $200 \mathrm{~cm}^{3} \mathrm{~min}^{-1}$, which is equivalent to an average pore velocity of $14 \mathrm{~cm} \mathrm{~d}^{-1}$. The flow during the September 1984 pulse ranged from about 60 to $200 \mathrm{~cm}^{3} / \mathrm{min}$. Moisture content varied with time and depth from 25 to $39 \%$ with an arithmetic average of about $28 \%$. 
Figs. 47 to 49 summarize the estimates for the flow and transport parameters of the August 1983, September 1984, and December 1984 pulses. The most relevant features observed from those pulses and their comparisons follow:

(1) The parameters, dispersion coefficient, and retardance factor for bromide track those for iodide very well as a function of depth. Any differences are well within the expected experimental and analytical errors.

(i) The retordance factor tends to approach 1.0 with an increase in depth. A value of about 1.0 is expected for conservative tracers, such as iodide and bromide. The tendency for the retardance factor to approach unity with an increase in depth agrees with the state-of-the-art issue that Fickian models (Eq. 1, for example) represent reality better at distances far from the source than at distances close to the source.

(3) The parameter estimates of the lower depths for both pulses are consistent with the expected dispersion coefficients and retardance factors. The deviation from those estimates at the upper levels of the caisson is greater for the August pulse than for the December pulse. Two major factors could account for the improvement in the parameter estimates for the latter pulse. One is an increase in the homogeneity of the soil characteristics through rearrangement of the soil matrix. The second is more uniformity in the tracer application of the December pulse: the tracers were distributed through 96 outlets in the December pulse and through only 64 outlets in the August pulse.

(4) Lithium shows a slightly higher stabilized retardation factor than iodide or bromide; however, its physical dispersion coefficient is well within the range of those for either iodide or bromide.

(5) Breakthrough predictions are quite satisfactory for all pulses. Although problems exist at the upper levels in the caisscns, the 
predictions get better as depth increases. Conservation of mass in the predictions is not effectively maintained. Reasons may be related to either the applicability of the model itself, the lack of spatial variability considerations, or both.

The retardation factors and dispersion coefficients for three iodide tracer pulses are shown as functions of caisson depth in Fig. 49. The results indicate that parameter estimates can be reproduced reasonably well for iodide transport for depths of 1 ill or greater. The unusually high retardation factors and dispersion coefficients above the 1-m depth probably result from nonuniform input of solutes at the soil surface. Unlike the other two pulses (Fig. 49), the pulse introduced in September was an unsteady-state pulse; parameter estimates for this pulse could not be made because the paraneter estination techniques used assumed steady-state flow conditions.

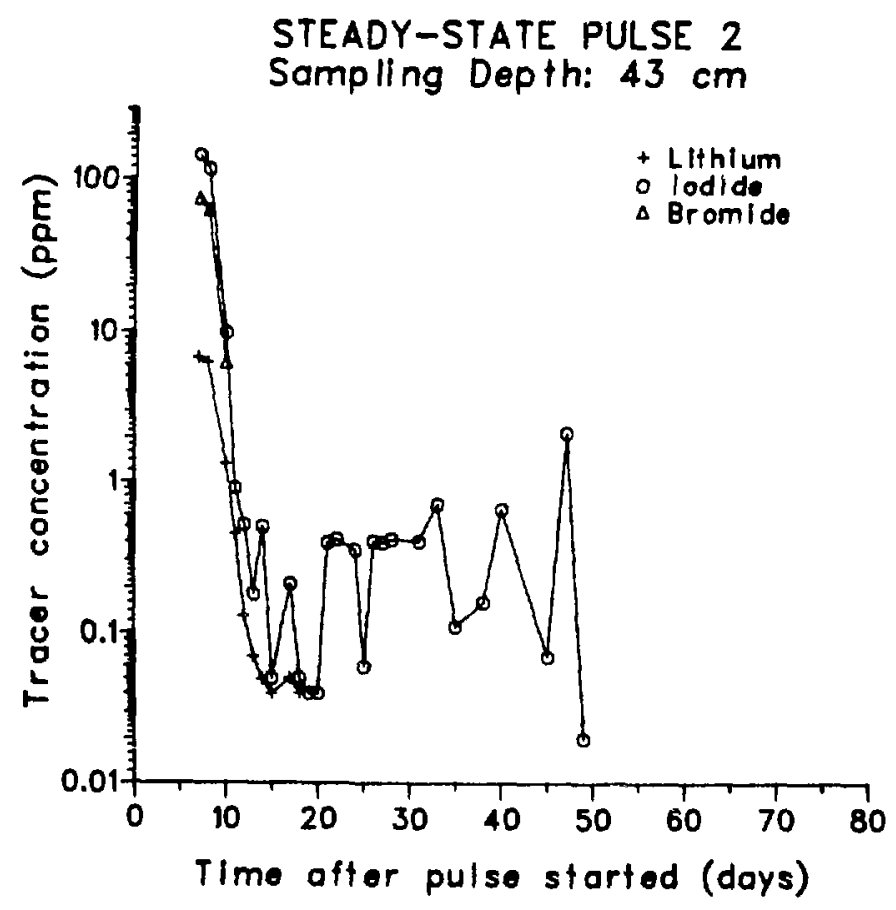

Fig. 35. Iodide, bromide, and lithium concentrations as a function of time for the vertical sampler emplaced at the 43-cm depth in Caisson B. 


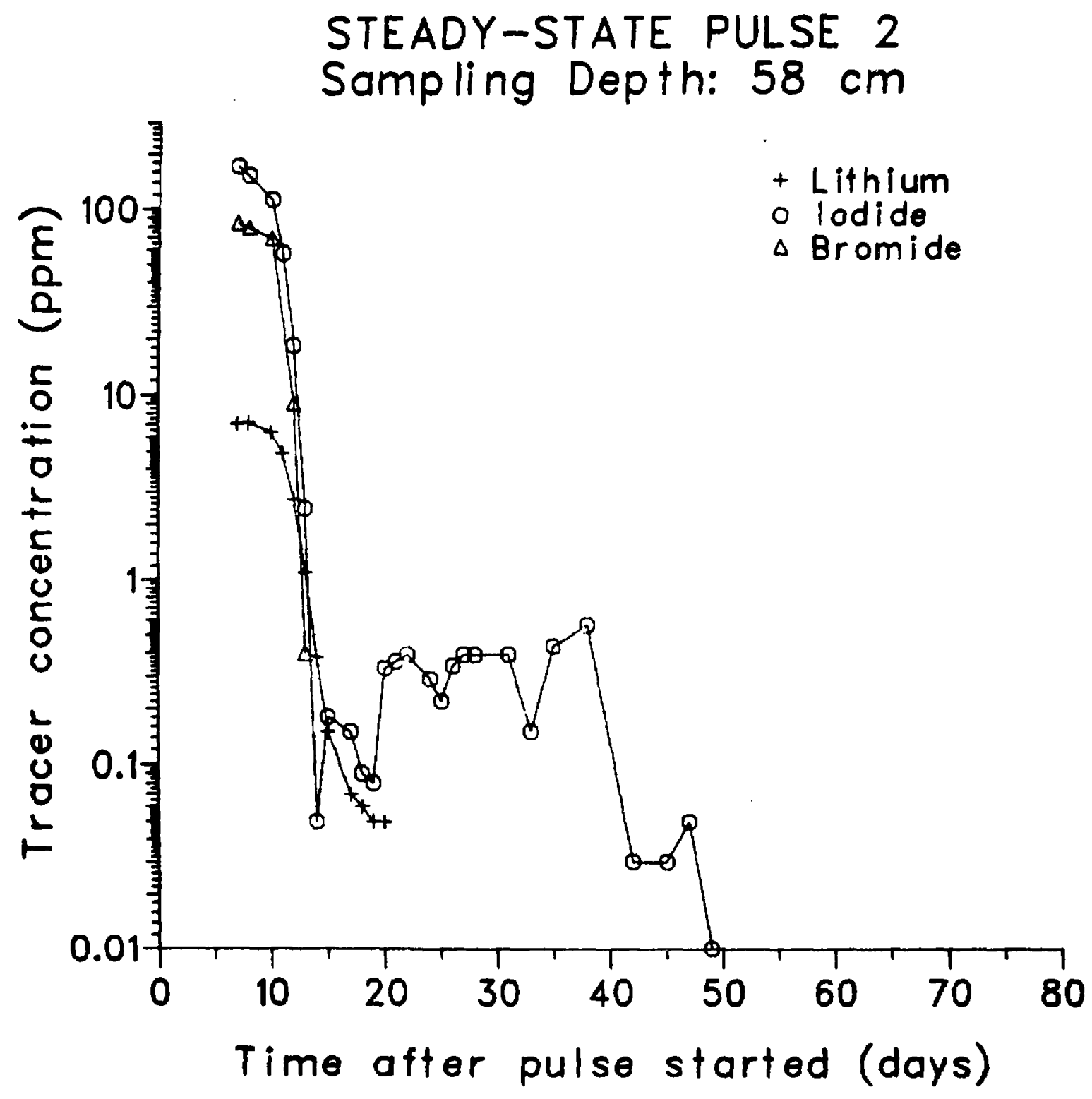

Fig. 36. Iodide, bromide, and lithium concentrations as a function of time for the vertical sampler emplaced at the $58-\mathrm{cm}$ depth in Caisson $B$. 


\section{STEADY-STATE PULSE 2 Sampling Depth: $73 \mathrm{~cm}$}

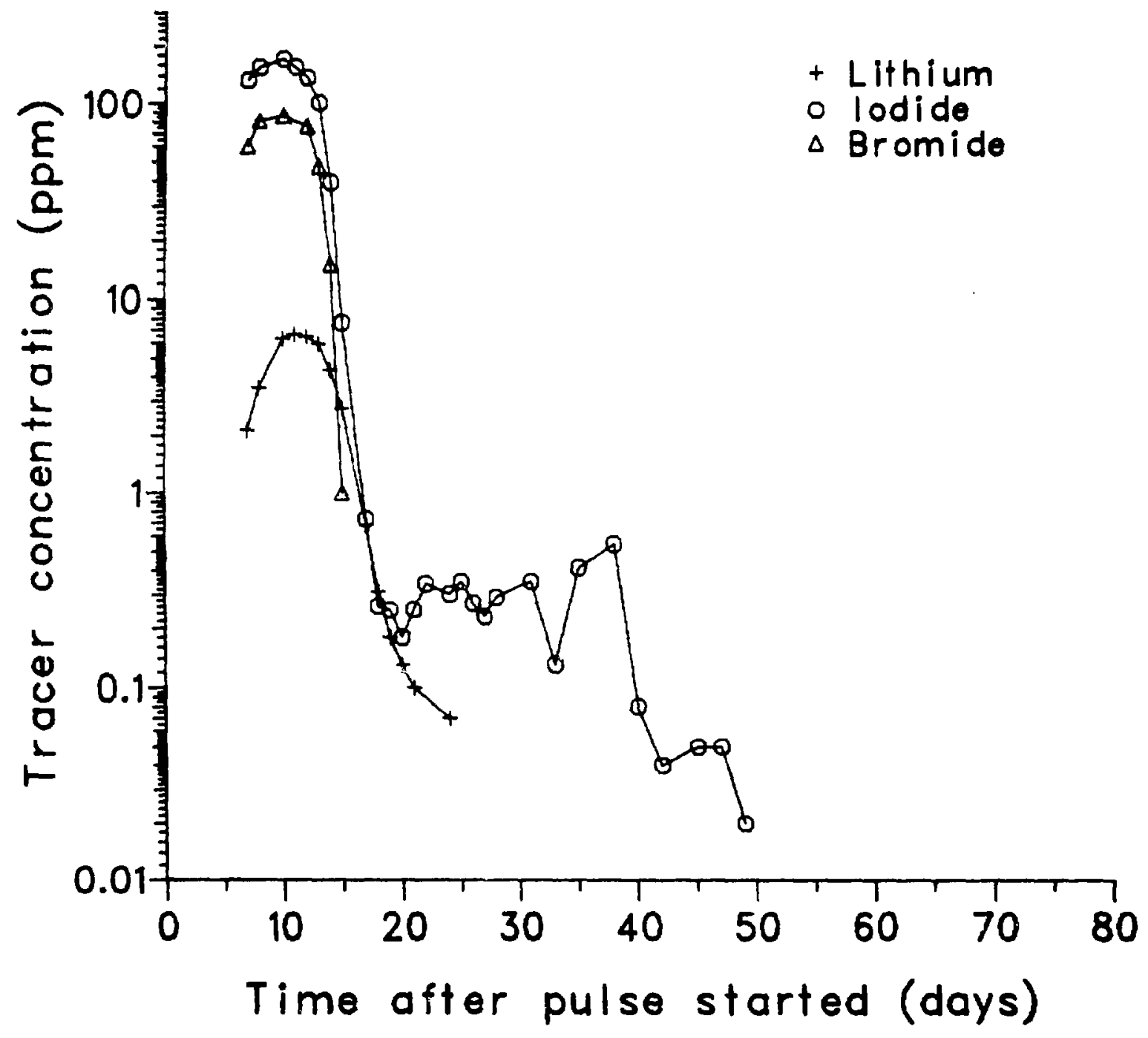

Fig. 37. Iodide, bromide, and lithium concentrations as a function of time for the vertical sampler emplaced at the $73-\mathrm{cm}$ depth in Caisson $B$. 


\section{STEADY-STATE PULSE 2 Sampling Depth: $88 \mathrm{~cm}$}

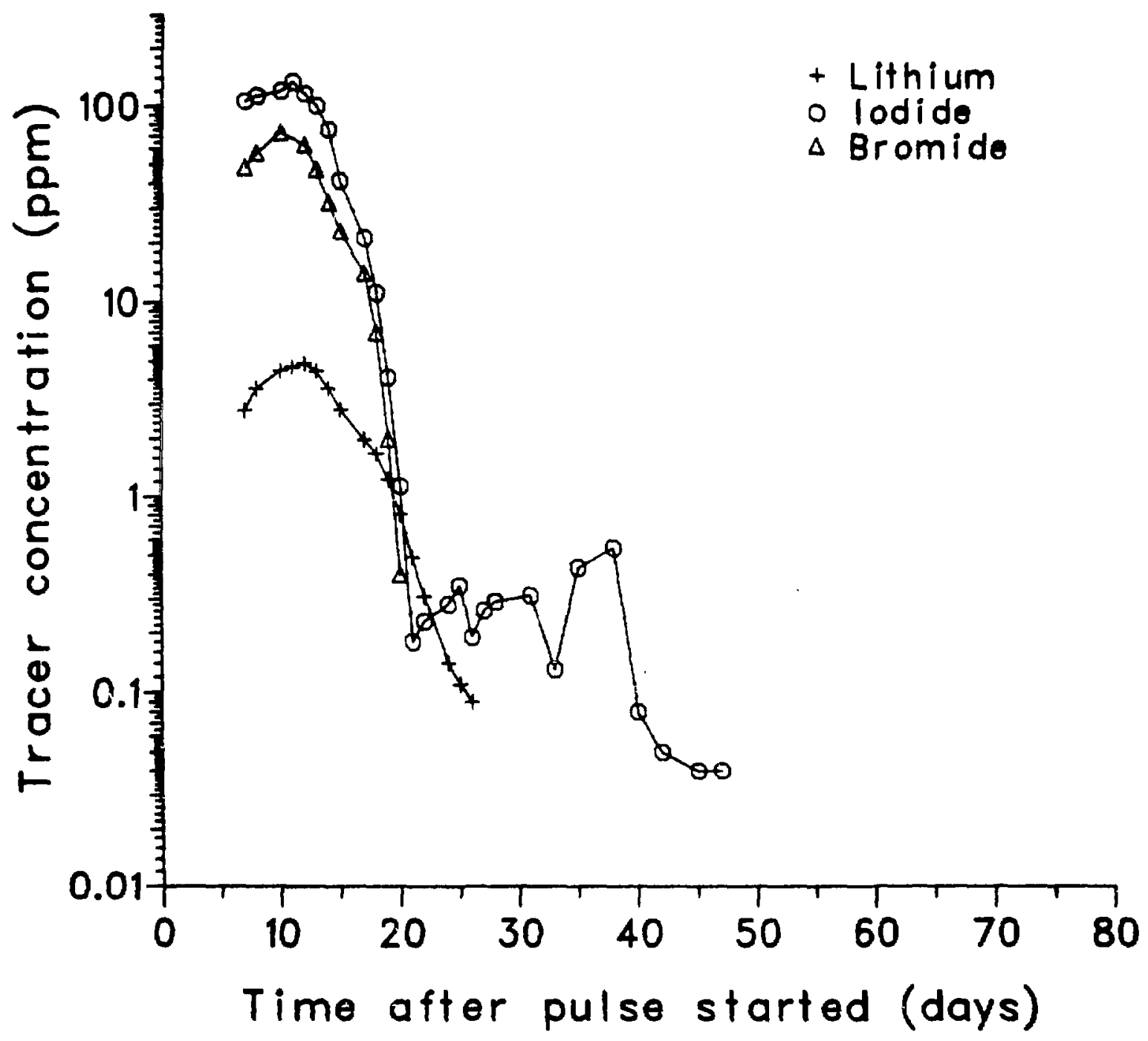

Fig. 38. Iodide, bromide, and lithium concentrations as a function of time for the vertical sampler emplaced at the 88-cm depth in Caisson B. 


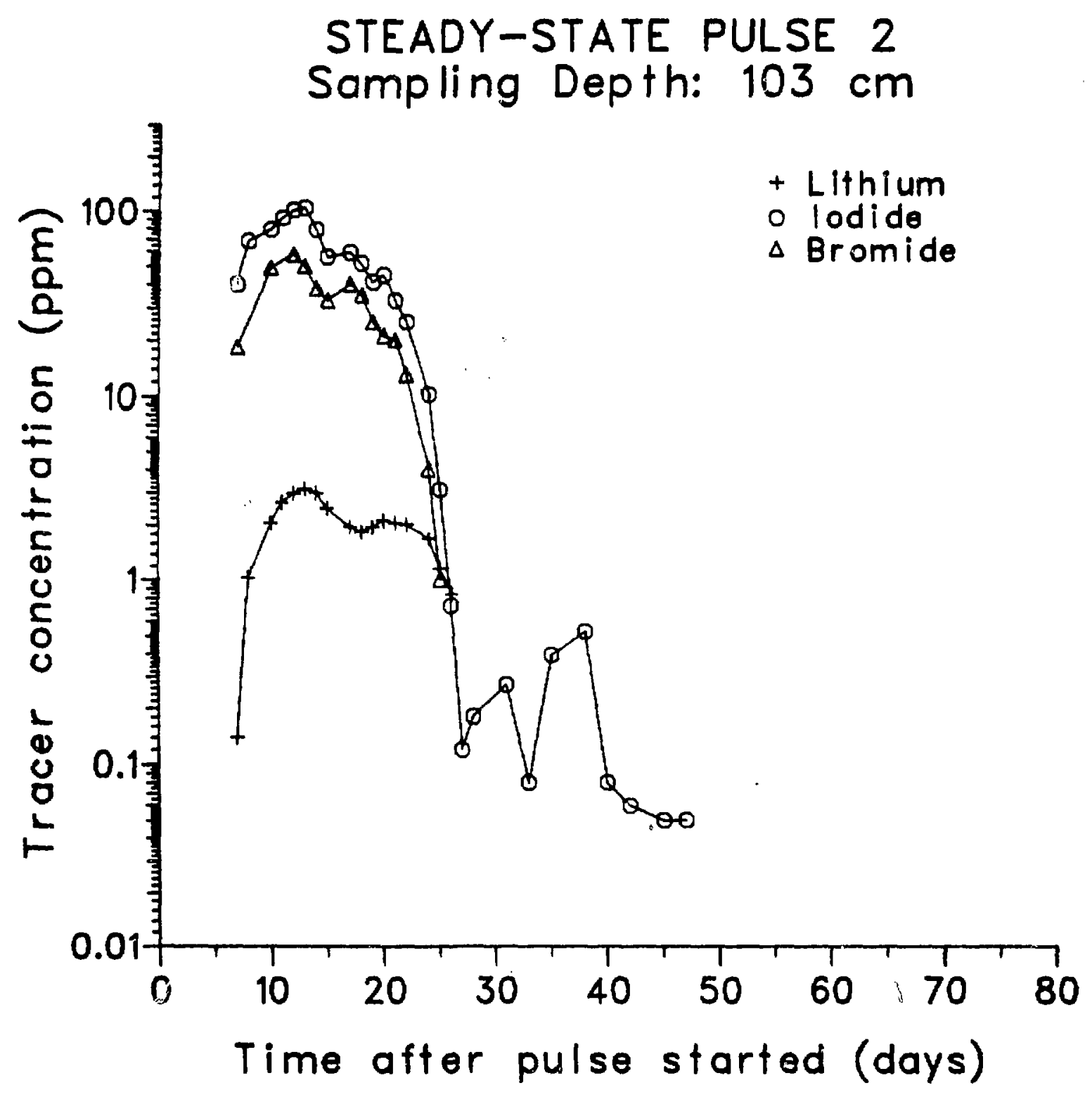

Fig. 39. Iodide, bromide, and lithium concentrations as a function of time for the vertical sampler emplaced at the 103-cm depth in Caisson $B$. 


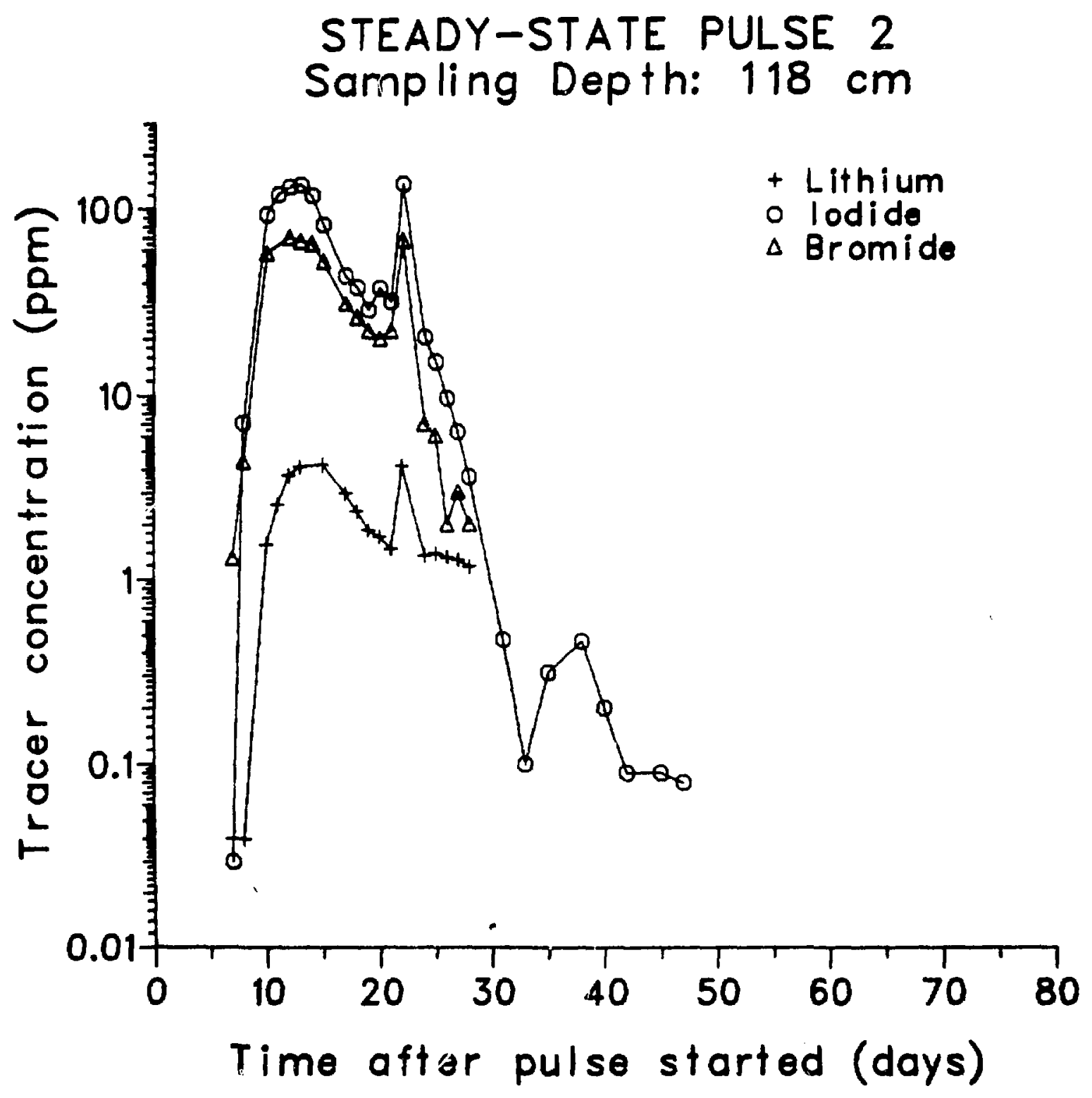

Fig. 40. Iodide, bromide, and lithium concentrations as a function of time for the vertical sampler emplaced at the 118-cm depth in Caisson B. 


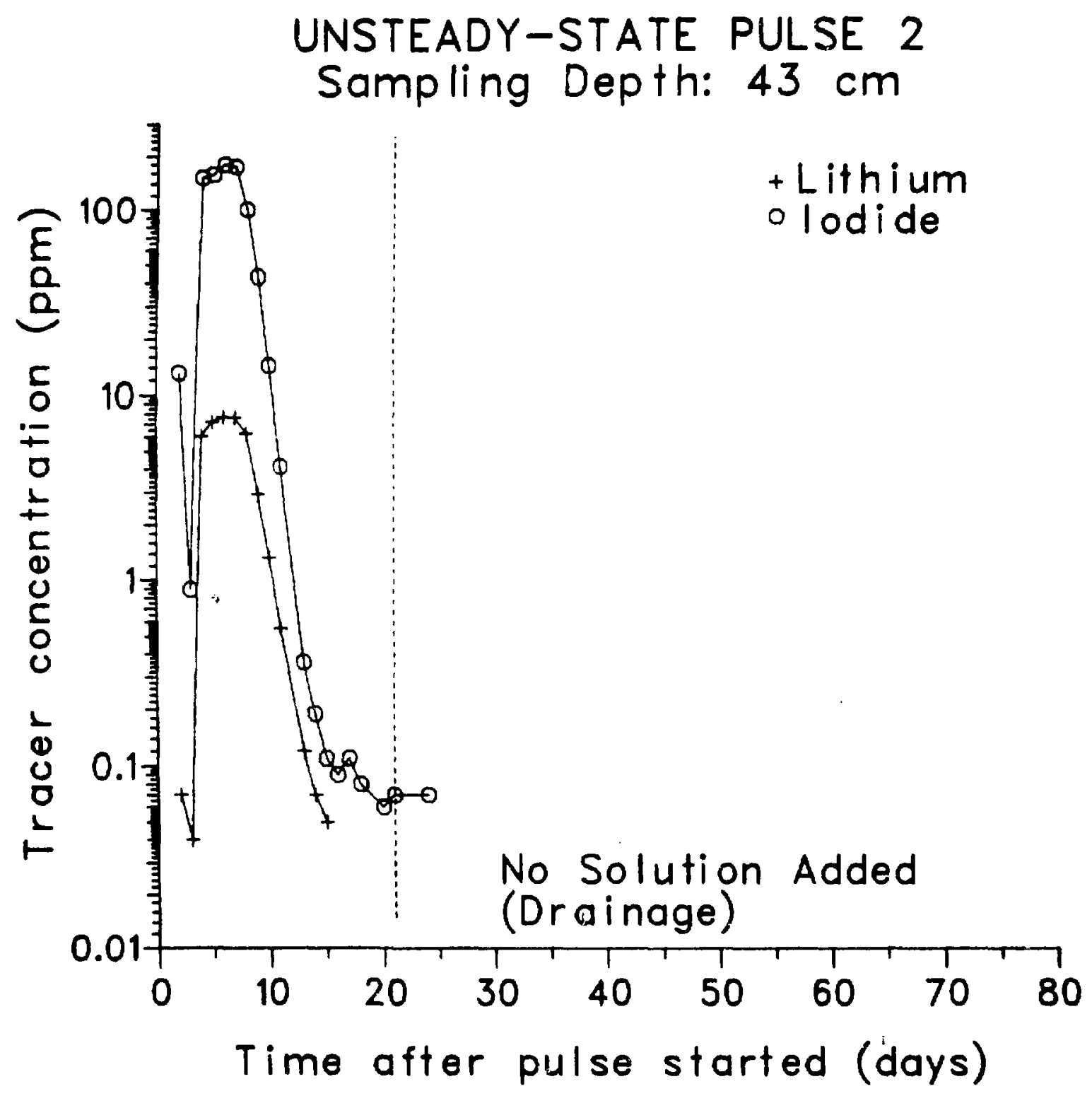

Fig. 41. Iodide and lithium concentrations as a function of time for the vertical sampler emplaced at the 43-cm depth in Caisson B. 


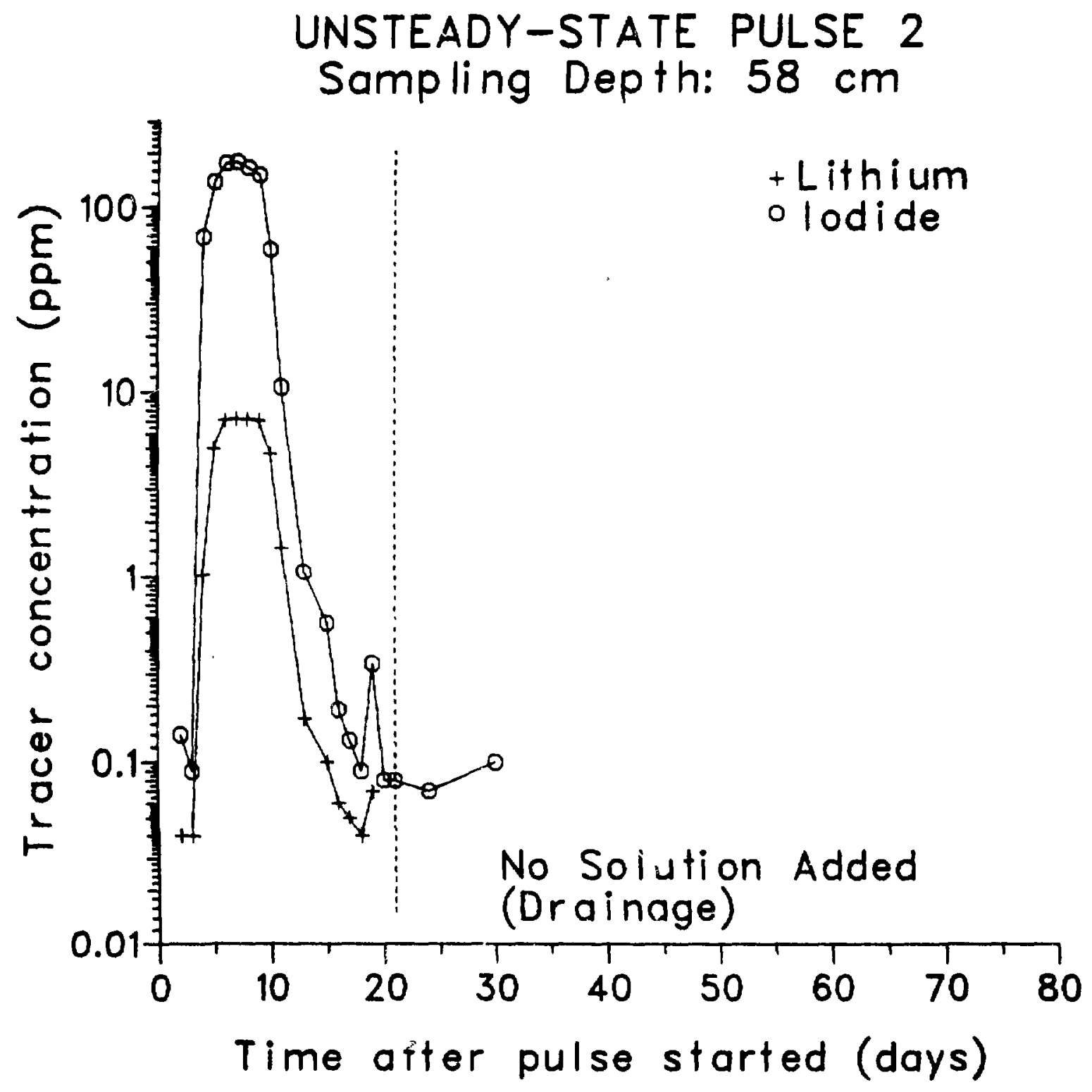

Fig. 42. Iodide and lithium concentrations as a function of time for the vertical sampler emplaced at the $58-\mathrm{cm}$ depth in Caisson $B$. 


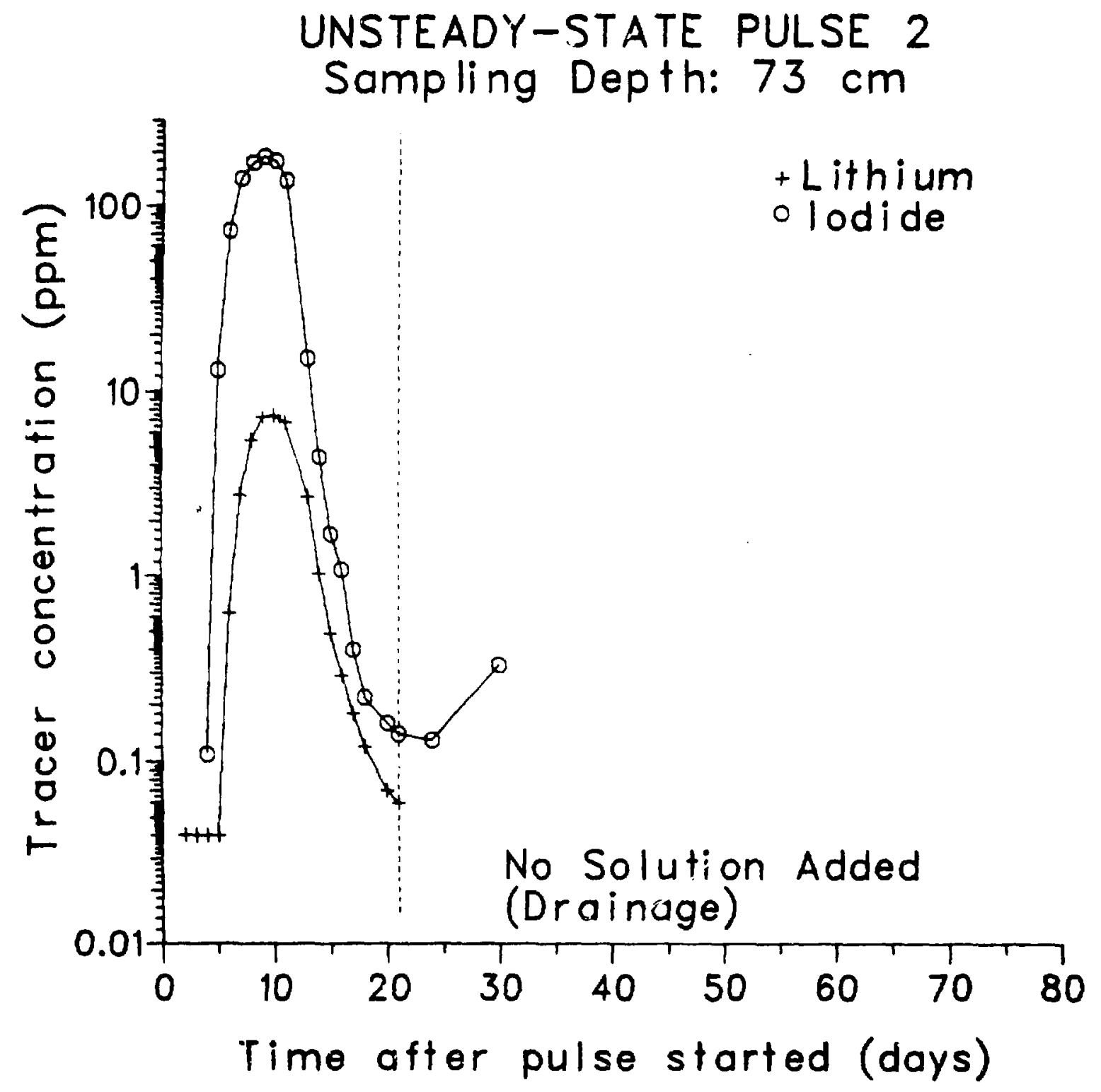

Fig. 43. Iodide and lithium concentrations as a function of time for the vertical sampler emplaced at the 73-cm depth in Caisson $B$. 


\section{UNSTEADY-STATE PULSE 2 Sampling Depth: $88 \mathrm{~cm}$}

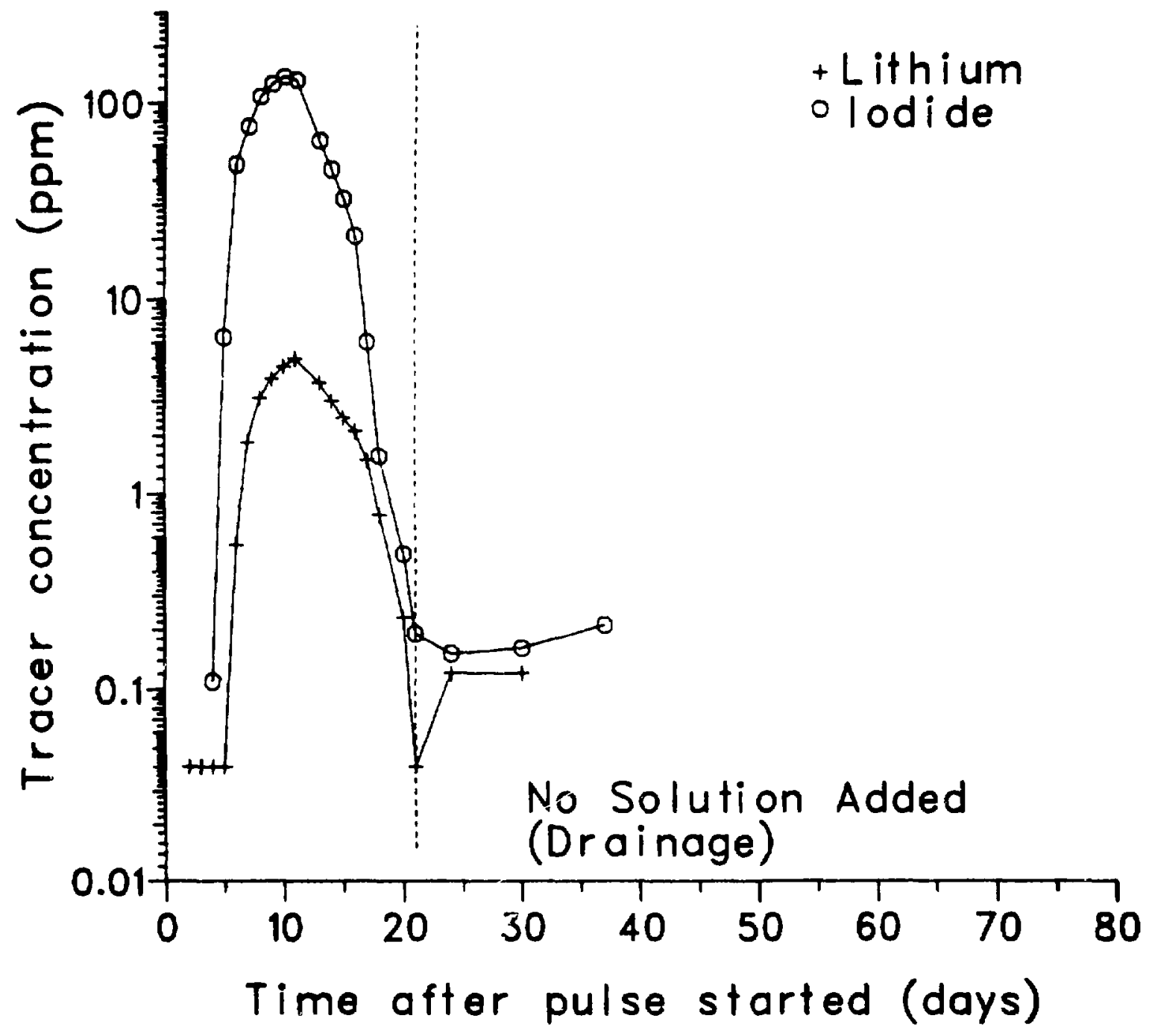

Fig. 44. Iodide and lithium concentrations as a function of time for the vertical sampler emplaced at the 88-cm depth in Caisson B. 


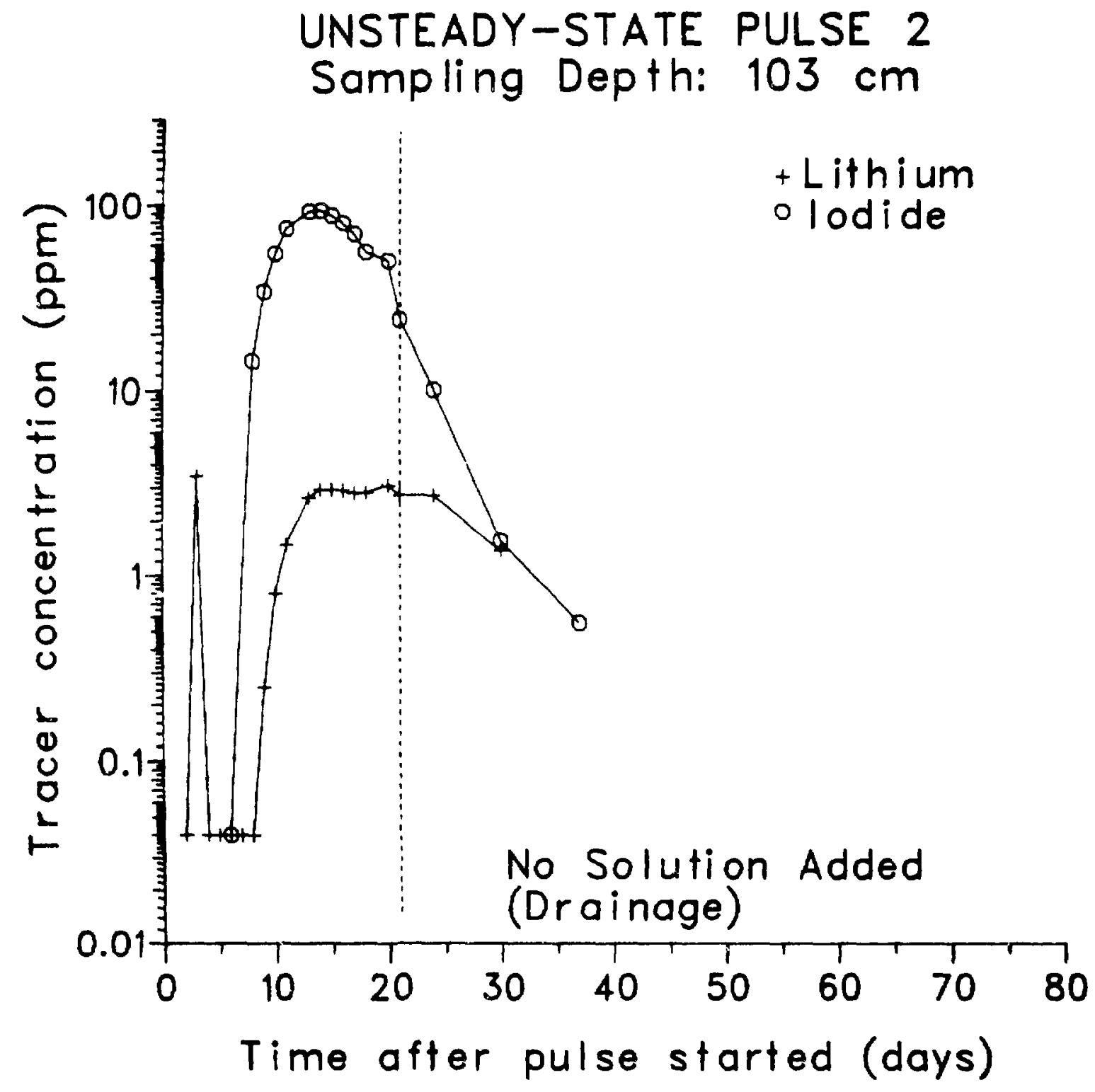

Fig. 45. Iodide and lithium concentrations as a function of time for the vertical sampler emplaced at the 103-cm depth in Caisson B. 


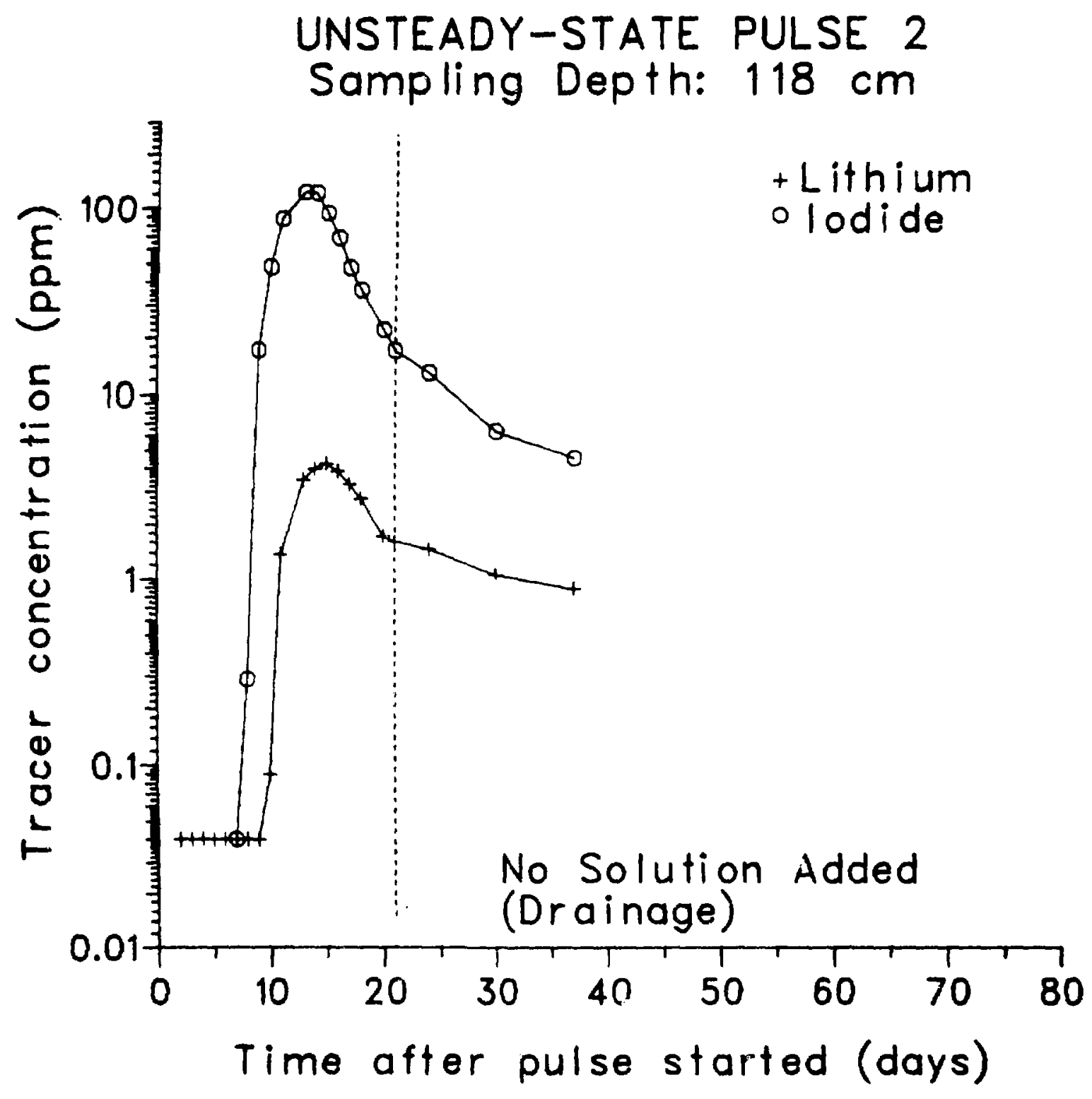

Fig. 46. Iodide and lithium concentrations as a function of time for the vertical sampler emplaced at the 118-cm depth in Caisson $B$. 
OPTIMAL PARAMETER ESTIMATES
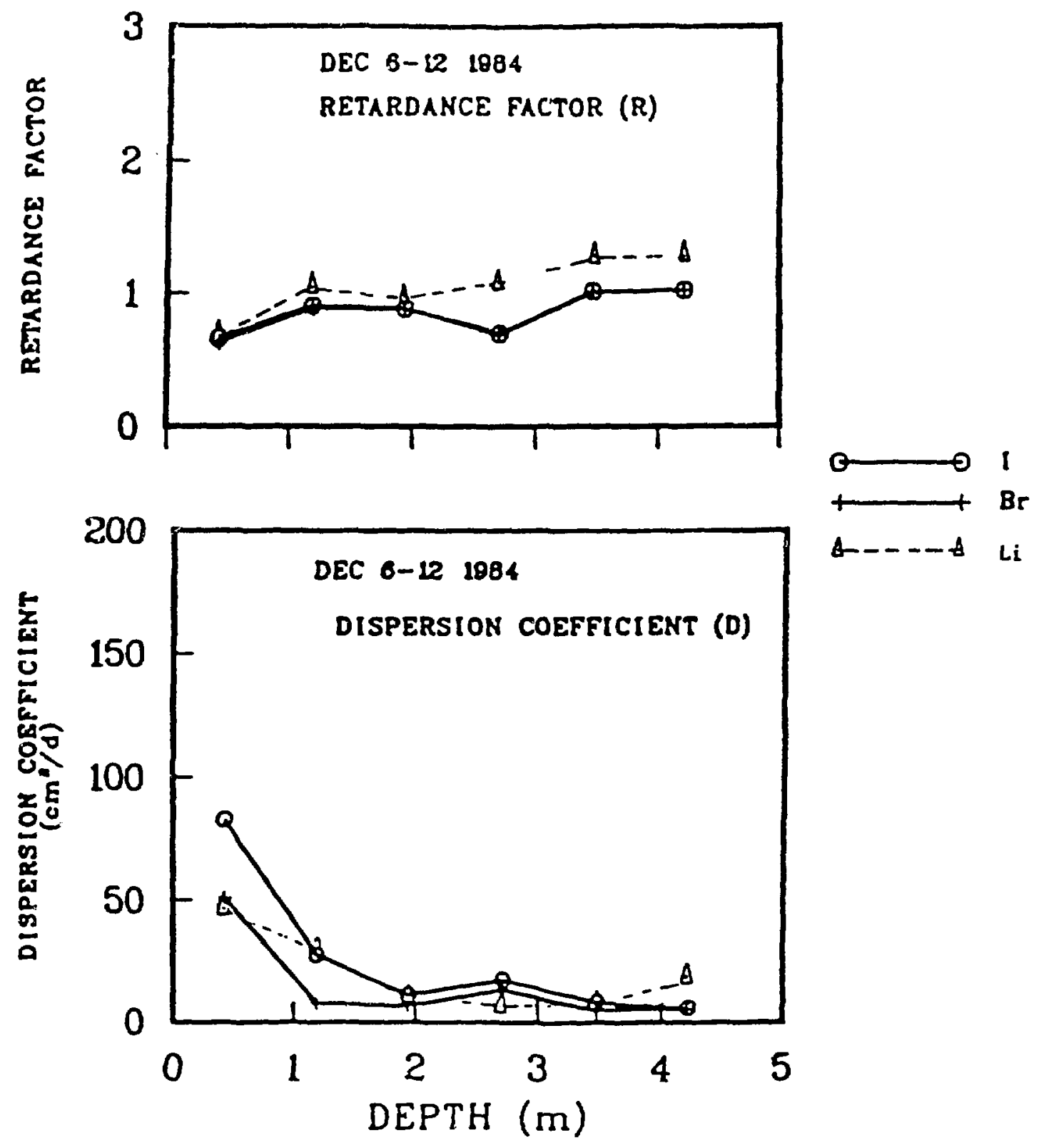

Fig. 47. Parameter estimates for three tracers as a function of depth in Caisson B (steady-state pulse). 
OPTIMAL PARAMETER ESTIMATES
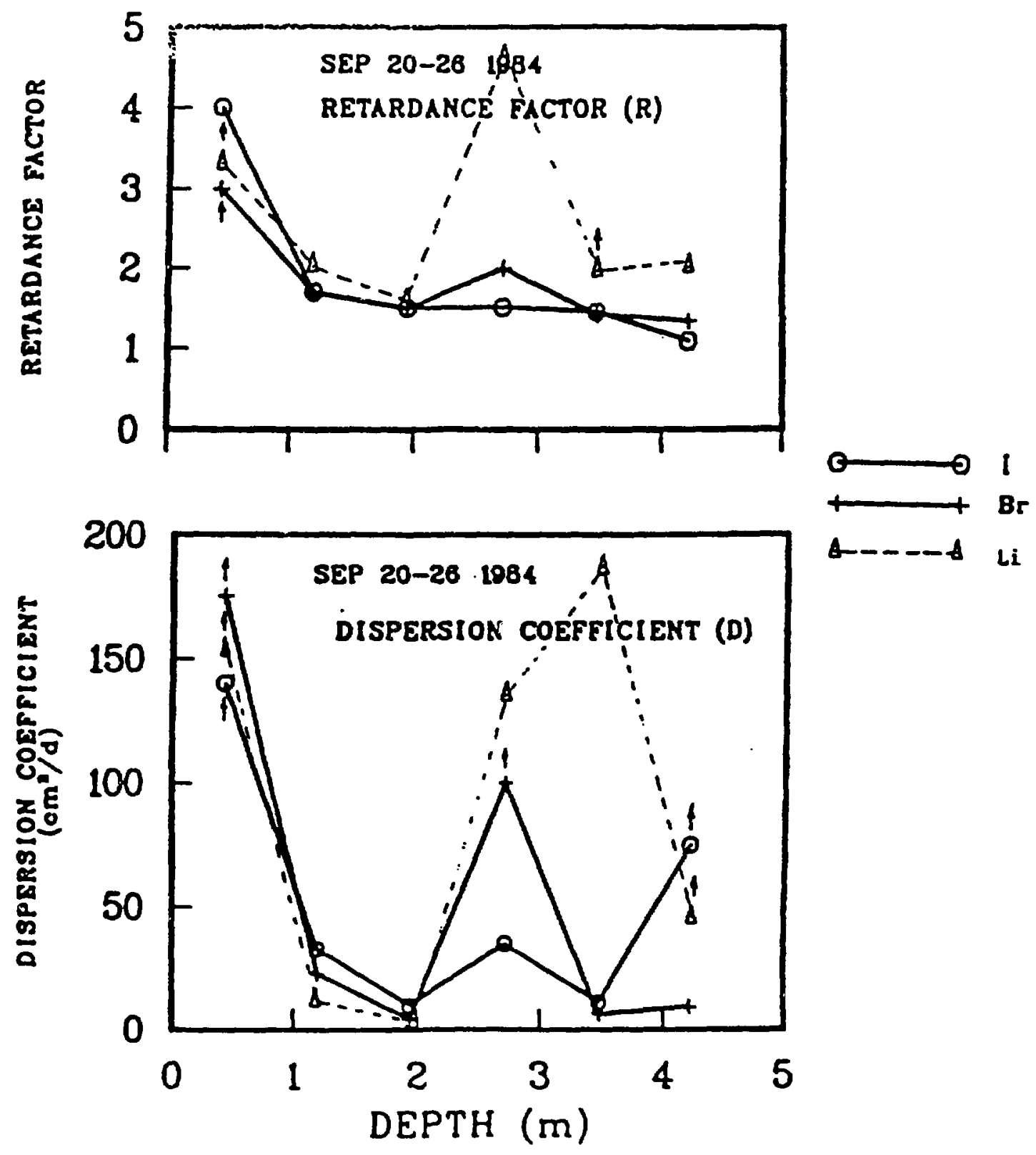

Fig. 48. Parameter estimates for three tracers as a function of depth in Caisson B (unsteady-state pulse). 

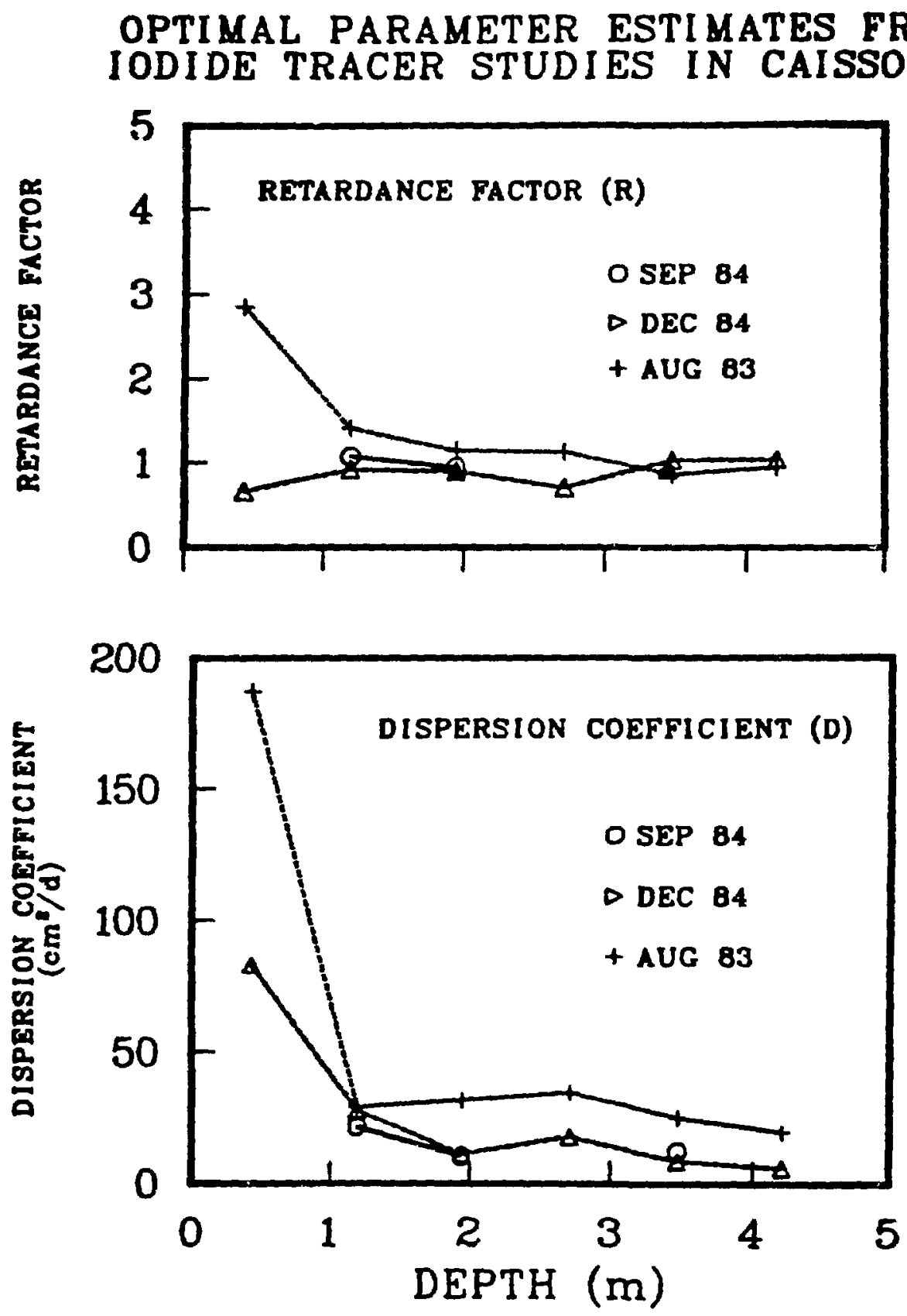

Fig. 49. Parameter estimates for iodide tracer studies in Caisson B. 


\section{FUTURE RESEARCH CONSIDERATIONS}

Since the field work for this experiment was concluded at the end of 1985 and most of the chemical assays were finished in September 1985, we have not performed extensive data analysis on the transport of iodide, bromide, and lithium in this document. However, the results of these field experiments are so impressive that we have convinced several funding agencies that coniderable modeling efforts should be expended on this data during 1986 . Basically, additional parameter estimates need to be made for all of the tracer data, except for the preliminary results shown in Figs. 47-49. The inflow/outflow tracer data need to be tabulated and analyzed, and final analytical work for the NRC program (cesium and strontium) needs to be completed, followed by extensive transport modeling.

In spite of these few loose ends, the unique field data sets included in this report can now be used to model chemical transport in unsaturated conditions for both steady-state and unsteady-state flow. Considerable information has been collected on the transport of iodide, bromide, and lithium under steady-state flow conditions that can be used to field-validate several current transport codes. Although this will be useful to the waste management community, the real challenge will be met in modeling the unsteadystate flow of these tracers with current transport codes, and finding their limitations.

Our preliminary findings were that iodide and bromide migration seem to be enhanced under unsteady-state flow conditions; while lithium transport demonstrated just the opposite pattern. This behavior, along witn similar NRC data on cesium and strontium transport, will provide real challenges to current transport codes, many of which will probably only work successfully under steady-state flow conditions. Because most transport in the field, such 
as liquid waste spills and leaching of buried wastes in landfills, probably occurs within the unsteady-state (unsaturated) flow regime, our ultimate goal is to be able to model chemical transport under these transient conditions. We believe that successful modeling of the tracer data included in this report will be a big step in this direction.

\section{REFERENCES}

Abeele, W. V., M. L. Wheeler, and B. W. Burton, "Geohydrology of Bandelier Tuff," Los Alamos Natiunal Laboratory report LA-8962-MS (October 1981).

Abeele, W. V., "Hydraulic Testing of Crushed Bandelier Tuff," Los Alamos National Laboratory report LA-10037-MS (March 1984).

Amoozegar-Fard, A., D. R. Nielsen, and A. W. Warrick, "Soil Solute Concentration Distribution for Spatially Varying Pore Water Velocities and Apparent Diffusion Coefficients," Soil Sci. Soc. Amer. Proc. 46, 3-9 (1982).

Arnold, E. M., A. W. Gee, and R. W. Nielsen, "Symposium on Unsteady Flow and Transport Modeling," Pacific Northwest Laboratory report, PNL-SA-10325, p. 351 (1982).

Arora, H. and R. Dayal, "Properties of Radioactive Wastes and Waste Containers, Quarterly Progress Report, October-December 1982, "Batellelle Northwest Laboratory report BNL-NUREG-32498 (1983).

Biggar, J. W. and D. R. Nielsen, "Improved Leaching Practices - Save Water, Reduce Drainage Problems," California Agriculture 16, 5 (1962).

Cokal, E. J., E. Stallings, R. Walker, J. W. Nyhan, W. L. Polzer, and E. H. Essington, "Apparatus for Groundwater Chemistry Investigations in Field Caissons," Proceedings of the Association of Groundwater Scientists and Engineers, Renc, Nevada, January 15-16, 1985, pp. 348-356.

DePoorter, G. L., "The Los Alamos Experimental Engineered Waste Burial Facility: Design Considerations and Preliminary Exnerimental Plan," in Waste Management ' 81, R. G. Post and M. E. Wacks, Eds. (University of Arizona, 1981), pp. 667-686.

Hespe, E. D., "Leach Testing of Immobilize's Radioactive Waste Solids, A Proposal for a Standard Method," Atomic Eriergy Review 9, 195-207 (1971).

Jackson, D.R., F. S. Brinkley, and E. A. Bondietti, "Extraction of Soil Water Using Cellulose-Acetate Hollow Fibers," Soil Science Soc. Amer. Proc. 40, 327-329 (1976). 
Jacobs, D. G., J. S. Epler, and R. R. Rose, "Identification of Te, nical Problems Encountered in the Shallow Land Burial of Low-Level Radioactive Wastes," Oak Ridge National Laboratory report ORNL/SUB-80/13619/1 (1980).

Miller, R. J., J. W. Biggar, and D. R. Nielsen, "Chloride Displacement in Panoche Clay Loam in Relation to Water Movement and Distribution," Water Resources Res. 1, 63-73 (1965).

Nielsen, D. R., J. W. Biggar, and J. N. Luthin, "Desalinization of Soils Under Controlled Unsaturated Flow Conditions," International Committee on Irrigation Drainage, bth Congress, New Delhi, India, Question 19, p. 15-24 (1965).

Nielsen, D. R., R. D. Jackson, J.W. Cory, and D. D. Evans, (eds.), "Soil Water," Amer. Soc. of Agronomy, Soil Sci. Soc. Am., Madison, Wisconsin (1972).

Nuclear Regulatory Agency, "10 CFR Parts 2, 19, 20, 21, 30, 40, 51, 61, 70, 73, and 170: Licensing Requirements for Land Disposal of Radioactive Waste," Federal Register 47, 57446-57482 (1982).

Onishi, Y., R. J. Serne, E. M. Arnold, C. E. Cowan, and F. L. Thompson, "Critical Review: Radionuclide Transport, Sediment Transport, and Water Quality Mathematical Modeling; and Radionuclide Adsorption/Desorption Mechanisms," Pacific Northwest Laboratory report PNL-2901 (1981;.

Simmons, C. S., "A Stochastic-Convective Ensemble Method for Representing Dispersive Transport in Groundwater," EPRI report CS-2558, Project 1406-1, Topical Report (1982).

US Department of Energy, Office of Nuclear Energy, "Spent Fuel and Radioactive Waste Inventories, Projections, and Characteristics, "US Department of Energy report DOE/NE-0017-1 (1982).

van Genuchten, M. Th. and W. J. Alves, "Analytical Solutions of the One-Dimensional Convective-Dispersive Solute Transport Equation," US Department of Agriculture, Tech. Bulletin 1661, p. 151 (1982).

Wierenga, P. J., "Solute Distribution Profiles Computed with Steady-State and Transient Water Movement Models," Soil Sci. Soc. Amer. Proc. 41, 1050-1055 (1977).

Wilson, L. G. and J. N. Luthin, "Effect of Air Flow Ahead of the Wetting Front on Infiltration," Soil Sci. 96, 136-143 (1963). 
APPENDIX A

VOLUMETRIC WATER CONTENT DATA BASE

FOR THE CAISSON B EXPERIMENTS 


\section{DATA BASE FOR THE CAISSON B EXPERIMENTS}

Six access tubes were emplaced in Caisson B, 1 icated at the Los Alamos EETF, to determine soil water content, using a neutron moisture gauge, as a function of soil depth and time. These access tubes were emplaced horizontally through access ports located in the instrument caisson and data were collected various distances from the center of Caisson $B$.

Access tubes $802,803,804,805,806$, and 807 were located 68, 145, 220, 295, 370, and $445 \mathrm{~cm}$, respectively, from the soil surface in Caisson B. Soil water content determinations were usually performed within each tube at $10-\mathrm{cm}$ increments from 0 to $130 \mathrm{~cm}$ from the center of Caisson $B$. 
SAMPLING
DATE

12APR 84

12APR 84

12 APR 84

12APR 84

12APR 84

12APR84

12APR84

12 APR 84

12 APR 84

12 APR 84

12 APR 84

12APR 84

12APR 84

12APR 84

1OMAY 84

1OMAY 84

1OMAY 84

10MAY84

IOMAY84

10MAY84

1OMAY 84

1OMAY 84

10MAY84

10MAY 84

I OMAY 84

10MAY84

10MAY 84

D6JUN84

06JUN84

06JUN84

06JUN84

06JUN84

06JUN84

06JUN84

06JUN84

06JUN84

06JUN84

06JUN84

06JUN84

06JUN84

25JUL84

25 JUL84

25 JUL84

25JUL84

25 JUL84

Di.PTH FROM
SURF:CE $(\mathrm{cm}$.

DISTANCE

FROM

CENTER

68

68

68

68

68

68

68

68

68

68

68

68

68

68

68

68

68

68

68

68

68

68

68

68

68

68

68

68

68

68

68

68

68

68

68

68

68

68

68

68

68

68

68

68

68
VOLUMETRIC

MOISTURE

CONTENT $(z)$

18.7

17.8

17.8

17.9

18.0

17.8

17.3

17.1

17.1

17.4

17.9

17.0

15.8

10.6

16.8

16.6

16.1

16.0

16.1

16.4

16.3

16.2

16. 1

16.1

16.1

15.6

14.3

29.5

27.6

27.6

27.5

26.8

27.3

26.1

26.6

26.8

26.3

25.7

25.6

24.3

29.5

27.9

27.8

27.7

27.5 


\section{SAMPLING \\ DATE}

25JUL84

25JUL84

25JUL84

25JUL84

25J UL84

25JUL84

25JUL84

25JUL84

09AUG84

09AUG84

O9AUG84

09AUG84

09AUG84

09AUG84

09AUG84

09AUG84

09AUG84

09AUG84

O9AUG84

09AUG84

O9AUG84

09AUG84

09AUG84

09AUG84

09AUG84

09AUG84

09AUG84

09AUG84

09AUG84

09AUG84

09AUG84

09AUG84

09AUG84

09AUG84

20AUG84

20AUG84

20AUG84

20AUG84

20AUG84

20AUG 84

20AUG84

20AUG84

20AUG84

20AUG84

20AUG84

\section{DEPTH FROM \\ SURFACE (cm.)

DISTANCE
FROM
CENTER

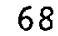

68

68

68

68

68

68

68

68

68

68

68

68

58

68

68

68

68

68

68

68

68

68

68

68

68

68

68

68

68

68

68

68

68

68

68

68

68

68

68

68

68

68

68

68
50

60

70

80

90

100

110

120

0

10

10

20

20

30

30

40

40

50

50

60

60

70

70

80

80

90

90

100

100

110

110

120

120

0

10

20

30

40

50

60

70

80

90

100
VOLUMETRIC MOISTURE

CONTENT ( 8 )

26.8

27.2

27.5

27.7

27.3

26.5

26.4

25.3

31.9

30.7

28.7

29.8

30.0

28.9

28.5

29.6

28.4

29.5

28.5

29.5

28. 2

29.2

29.6

28.6

29.2

28.2

29.0

28.0

27.5

28.4

27.7

28.7

26.3

27.2

33.5

31.8

30.7

31.4

30.7

30.2

30.5

31.0

30.7

30.5

29.6 


\begin{tabular}{|c|c|c|c|}
\hline $\begin{array}{l}\text { SAMPLING } \\
\text { DATE }\end{array}$ & $\begin{array}{l}\text { DEPTH FROM } \\
\text { SURFACE }(\mathrm{cm} .)\end{array}$ & $\begin{array}{l}\text { DISTANCE } \\
\text { FROM } \\
\text { CENTER }\end{array}$ & $\begin{array}{l}\text { VOLUMETRIC } \\
\text { MOISTURE } \\
\text { CONTENT }\end{array}$ \\
\hline $20 A \cup G 84$ & 68 & 110 & 29.1 \\
\hline $20 A U G 84$ & 68 & 120 & 28.6 \\
\hline 04 SEP84 & 68 & 0 & 32.1 \\
\hline 04 SEP 84 & 68 & 10 & 30.0 \\
\hline $04 \operatorname{SEP} 84$ & 68 & 20 & 30.1 \\
\hline 04 SEP 84 & 68 & 30 & 30.0 \\
\hline 04SEP84 & 68 & 40 & 29.5 \\
\hline 04SEP84 & 68 & 50 & 29.3 \\
\hline 04 SEP84 & 68 & 60 & 29.2 \\
\hline 04SEP84 & 68 & 70 & 29.5 \\
\hline 04 SEP 84 & 68 & 80 & 29.5 \\
\hline 04 SEP 84 & 68 & 90 & 28.5 \\
\hline O4SEP 84 & 68 & 100 & 27.9 \\
\hline 04SEP34 & 68 & 110 & 28.2 \\
\hline 04SEP 84 & 68 & 120 & 26.4 \\
\hline $18 \mathrm{SEP} 84$ & 68 & 0 & 29.3 \\
\hline $18 \mathrm{SEP} 84$ & 68 & 10 & 27.7 \\
\hline $18 \mathrm{SEE} 84$ & 68 & 20 & 27.4 \\
\hline IBSEPS: & 68 & 30 & 27.3 \\
\hline 18 SEP 84 & 68 & 40 & 27.2 \\
\hline 18 SEP84 & 68 & 50 & 27.4 \\
\hline 18SE.P 84 & 68 & 60 & 26.9 \\
\hline 18 SEF 84 & 68 & 70 & 27.2 \\
\hline 18SEP84 & 68 & 80 & 27.0 \\
\hline 18 SEP 84 & 68 & 90 & 26.4 \\
\hline 18SEP84 & 68 & 100 & 26.2 \\
\hline 18SEP84 & 68 & 110 & 25.5 \\
\hline 18SEP84 & 68 & 120 & 24.5 \\
\hline 020CT84 & 68 & 0 & 28.8 \\
\hline $020 \mathrm{CT} 84$ & 68 & 10 & 27.2 \\
\hline 020CT 84 & 68 & 20 & 27.0 \\
\hline 020CT84 & 68 & 30 & 26.9 \\
\hline 020CT84 & 68 & 40 & 26.8 \\
\hline $020 C T 84$ & 68 & 50 & 26.9 \\
\hline $020 \mathrm{CT} 84$ & 68 & 60 & 26.6 \\
\hline 020CT84 & 68 & 70 & 26.4 \\
\hline 020CT84 & 68 & 80 & 26.4 \\
\hline $020 \mathrm{CT} 84$ & 68 & 90 & 25.9 \\
\hline 020CT84 & 68 & 100 & 25.3 \\
\hline $020 \mathrm{CT} 84$ & 68 & 110 & 25.0 \\
\hline $020 C T 84$ & 68 & 120 & 24.1 \\
\hline $180 \mathrm{CT} 84$ & 68 & 0 & 30.0 \\
\hline $180 \mathrm{CT} 84$ & 68 & 10 & 27.9 \\
\hline $180 C T 84$ & 68 & 20 & 28.2 \\
\hline $180 \mathrm{CT} 84$ & 68 & 30 & 27.4 \\
\hline
\end{tabular}




\section{SAMPLING \\ DATE}

$180 \mathrm{CT} 84$

$180 \mathrm{CT} 84$

$180 \mathrm{CT} 84$

$180 \mathrm{CT} 84$

i $80 \mathrm{CT} 84$

$180 \mathrm{CT} 84$

$180 \mathrm{CT} 84$

$180 \mathrm{CT} 84$

$180 \mathrm{CT} 84$

$310 \mathrm{CT} 84$

$310 \mathrm{CT} 84$

$310 \mathrm{CT} 84$

$310 \mathrm{CT} 84$

$310 \mathrm{CT} 84$

$310 \mathrm{CT} 84$

$310 \mathrm{CT} 84$

$310 \mathrm{CT} 84$

$310 \mathrm{CT} 84$

$310 C T 84$

$310 \mathrm{CT} 84$

310 TT 84

310CT 84

28 NOV 84

28 NOV 84

28NOV 84

28 NOV 84

28NOV84

28 NOV 84

28 INOV 84

28 NOV 84

28 NOV 84

28NOV 84

28NOV 84

28NOV84

28NOV84

03JAN85

03JAN85

03.JAN85

17JAN8 5

17JAN85

17 JAN 85

04FEB85

04FEB8 5

04FEB 85

20FEB 85

DEPTH FROM
SURFACE (cm.)

68

68

68

68

68

68

68

68

68

68

68

68

68

68

68

68

68

68

68

68

68

68

68

68

68

68

68

68

68

68

68

68

68

68

68

68

68

68

68

68

68

68

68

68

68

$\begin{array}{ll}\text { DISTANCE } & \text { VOLUMETRIC } \\ \text { FROM } & \text { MOISTURE } \\ \text { CENTER } & \text { CONTENT ( } 8)\end{array}$

27.0

27.0

26.4

27.5

27.5

26.2

26.2

26.2

25.6

29.1

27.6

27.9

27.0

27.2

26.8

26.8

26.8

26.7

26.4

24.9

25.1

24.3

30.2

28.3

27 .

28.0

27.6

27.6

27.7

27.4

27.8

27.4

26.5

26.6

25.6

28.3

26.2

24.8

30.5

29.4

27.9

25.8

24.1

22.1

29.5 
SAMPLING

DATE

20FEB 85

$20 F E B 85$

O6MAR8 5

OGMAR8 5

06MAR 85

21MAR 85

21MAR85

21 MAR 85

OIAPR 85

O1APR85

01.APR 85

C8APR 5

08APR 85

D8APR 85

25APR 85

25APR 85

25APR 85

29APR 85

29APR 85

29APR 8

30APR 85

30 APR 85

30APR 85

01MAY85

01MAY85

01MAY85

$02 \mathrm{MAY} 85$

02MAY85

02 MAY85

03 MAY 85

03 MAY85

03 MAY 85

06MAY85

06MAY85

06MAY85

$08 M A Y 85$

08MAY85

O8MAY85.

10MAY85

10MAY85

10MAY85

13MAY85

13MAY85

13 MAY85

15MAY85
DEFTH FROM

SURFACE ( $\mathrm{cm}$.

68

68

68

68

68

68

68

68

68

68

68

68

68

58

68

68

68

68

68

68

68

68

68

68

68

68

68

68

68

68

68

68

63

68

68

68

68

68

68

68

68

68

68

68

68
DISTANCE VOLUMETRIC

FROM MOISTURE.

CENTER

CONTENT ( $\%$ )

27.9

26.1

31.0

29.1

27.1

31.4

29.5

28.1

33.4

31.0

29.0

30.9

29.0

27.2

29.9

27.7

25.8

29.6

27.7

25.7

27.5

26.0

22.6

26.0

24.7

21.4

25.2

23.4

19.4

24.3

23.2

18.6

23.1

21.9

19.0

22.5

21.2

17.3

21.8

20.7

17.4

21.5

20.2

18.1

21.0 


\section{SAMPLING DATE}

15MAY85

$15 \mathrm{MAY} 85$

17MAY85

17MAY85

17MAY85

21MAY85

21MAY85

21MAY85

23MAY85

23MAY85

23MAY85

28 MAY85

28MAY85

28MAY85

30MAY85

30MAY85

30MAYY 85

04JUN85

04JUN85

04JUN85

12JUN8 5

12JUN85

12JUN85

$18 J U N 85$

18JUN85

18 JUN85

25JUN85

25JUN85

25JUN85

02JUL85

02JUL85

02JUL85

11JUL85

11 JUL85

11JUL85

12JUL85

12JUL85

12JUL85

13JUL85

13JUL85

13JUL85

14 JUL85

14JUL85

14JUL85

15JUL85

\section{DEPTH FROM \\ SURFACE (cm.)

DISTANCE
FROM
CENTER

\section{8}

68

68

68

68

68

68

68

68

68

68

68

68

68

68

68

68

68

68

68

68

68

68

68

68

68

68

68

68

68

68

68

68

68

68

68

68

68

68

68

68

68

68

68

68
VOLUMETRIC

MOISTURE

CONTENT (\%)

20.0

17.9

20.8

19.7

16.9

20.3

19.5

17.5

20.5

18.9

17.4

20.1

18.8

16.9

19.6

18.8

16.5

18.9

18.2

16.0

18.0

17.5

15.8

18.0

17.0

14.7

17.2

16.7

15.0

17.0

16.2

14.8

16.5

15.9

14.0

23.0

20.5

15.5

26.9

24.5

21.5

28.1

25.7

23.0

27.7 


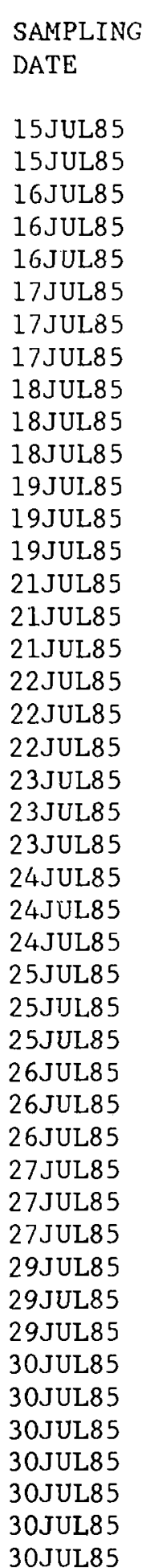

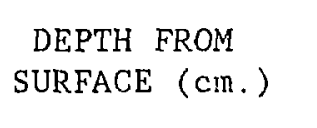

68

68

68

68

68

68

68

68

68

68

68

68

68

68

68

68

68

68

68

68

68

68

68

68

68

68

68

68

68

68

68

68

68

68

68

68

68

68

68

68

68

68

68

68

68
DISTANCE VOLUMETRIC FROM MOISTURE

CENTER

CONTENT

25.9

22.8

27.8

25.8

20.6

27.5

25.4

23.3

28.3

25.7

23.7

28.2

25.7

20.6

28.2

25.7

22.8

28.5

25.8

23.8

28.7

25.7

22.9

28.6

25.9

22.1

29.0

26.4

23.1

28.9

26.5

23.5

29.0

26.3

24.3

28.2

26.2

23.5

28.7

26.2

28.7

27.7

23.8

27.5

22.5 


\begin{tabular}{|c|c|c|c|}
\hline $\begin{array}{l}\text { SAMPLING } \\
\text { DATE }\end{array}$ & $\begin{array}{c}\text { DEPTH FROM } \\
\text { SURFACE (cm.) }\end{array}$ & $\begin{array}{l}\text { DISTANCE } \\
\text { FROM } \\
\text { CENTER }\end{array}$ & $\begin{array}{l}\text { VOLUMETRIC } \\
\text { MOISTURE } \\
\text { CONTENT ( })\end{array}$ \\
\hline 02AUG85 & 68 & 0 & 28.7 \\
\hline 02AUG85 & 68 & 60 & 26.9 \\
\hline 02 AUG 85 & 68 & 120 & 23.4 \\
\hline 04AUG85 & 68 & 0 & 28.9 \\
\hline 04AUG85 & 68 & 60 & 26.6 \\
\hline 04AUG85 & 68 & 120 & 24.2 \\
\hline 06AUG85 & 68 & 0 & 29.2 \\
\hline 06AUG85 & 68 & 60 & 26.9 \\
\hline 06AUG85 & 68 & 120 & 25.0 \\
\hline 09AUG85 & 68 & 0 & 28.6 \\
\hline 09AUG85 & 68 & 60 & 26.6 \\
\hline 09AUG85 & 68 & 120 & 24.6 \\
\hline 14AUG85 & 68 & 0 & 29.0 \\
\hline 14AUG 85 & 68 & 60 & 26.8 \\
\hline 14AUG85 & 68 & 120 & 24.3 \\
\hline 16AUG85 & 68 & 0 & 28.5 \\
\hline 16AUG85 & 68 & 0 & 29.0 \\
\hline 16AUG85 & 68 & 60 & 26.9 \\
\hline 16AUG85 & 68 & 60 & 26.0 \\
\hline 16AUG85 & 68 & 120 & 25.2 \\
\hline 21AUG85 & 68 & 0 & 29.3 \\
\hline 21AUG85 & 68 & 60 & 26.5 \\
\hline 21AUG85 & 68 & 120 & 24.6 \\
\hline 23AUG85 & 68 & 0 & 29.0 \\
\hline 23AUG85 & 68 & 60 & 27.1 \\
\hline 23AUG85 & 68 & 120 & 24.8 \\
\hline 27AUG85 & 68 & 0 & 29.1 \\
\hline 27AUG85 & 68 & 60 & 27.0 \\
\hline 27AUG85 & 68 & 120 & 24.5 \\
\hline 30AUG85 & 68 & 0 & 29.7 \\
\hline 30AUG85 & 68 & 60 & 27.0 \\
\hline $30 A U G 85$ & 68 & 120 & 24.6 \\
\hline 03SEP85 & 68 & 0 & 29.2 \\
\hline 03SEP85 & 68 & 60 & 27.0 \\
\hline 03SEP85 & 68 & 120 & 24.8 \\
\hline 09SEP85 & 68 & 0 & 29.1 \\
\hline 09SEP85 & 68 & 60 & 27.6 \\
\hline 09SEP85 & 68 & 120 & 24.8 \\
\hline 18SEP85 & 68 & 0 & 29.3 \\
\hline 18SEP85 & 68 & 60 & 27.7 \\
\hline 18SEP85 & 68 & 120 & 23.4 \\
\hline 30SEP85 & 68 & 0 & 29.5 \\
\hline 30 SEP85 & 68 & 60 & 27.5 \\
\hline 30SEP85 & 68 & 120 & 25.1 \\
\hline 090СТ85 & 68 & 0 & 29.0 \\
\hline
\end{tabular}


SAMPLING
DATE

$090 \mathrm{CT} 85$

$090 \mathrm{CT} 85$

$160 \mathrm{CT} 85$

$160 \mathrm{CT} 85$

$160 \mathrm{CT} 85$

$230 \mathrm{CT} 85$

$230 \mathrm{CT} 85$

$230 \mathrm{C}^{\mathrm{m}}+85$

$300 \mathrm{CT} 85$

$300 \mathrm{CT} 85$

$300 \mathrm{CT} 85$

06 NOV 85

06NOV85

06 NOV 85

DEPTH
SURFACE
68
68
68
68
68
68
68
68
68
68
68
68
68
68

DISTANCE VOLUMETRIC

FROM MOISTURE

CENTER CONTENT ( 8 )

$68 \quad 60$

120

0

60

120

0

60

120

0

60

120

0

60

120
27.9

24.0

30.1

26.6

24.8

28.7

27.4

26.2

29.0

27.0

25.3

29.7

27.7

24.8 


\begin{tabular}{|c|c|c|c|}
\hline $\begin{array}{l}\text { SAMPLING } \\
\text { DATE }\end{array}$ & $\begin{array}{c}\text { DEPTH FROM } \\
\text { SURFACE }(\mathrm{cm} .)\end{array}$ & $\begin{array}{l}\text { DISTANCE } \\
\text { FROM } \\
\text { GENTER }\end{array}$ & $\begin{array}{l}\text { VOLUMETRIC } \\
\text { MOISTURE } \\
\text { CONTENT. }(8)\end{array}$ \\
\hline 10MAY84 & 145 & 0 & 19.5 \\
\hline IOMAY84 & 145 & 10 & 18.3 \\
\hline 10MAY84 & 145 & 20 & 18.3 \\
\hline 10MAY84 & 145 & 30 & 18.4 \\
\hline 10MAY84 & 145 & 40 & 18.6 \\
\hline 10MAY84 & 145 & 50 & 18.5 \\
\hline 10MAY84 & 145 & 60 & 18.4 \\
\hline 10MAY84 & 145 & 70 & 18.1 \\
\hline 10MAY 84 & 145 & 80 & 17.5 \\
\hline 10MAY84 & 145 & 90 & 17.7 \\
\hline 10MAY84 & 145 & 100 & 17.4 \\
\hline 10MAY84 & 145 & 110 & 17.0 \\
\hline 10MAY84 & 145 & 120 & 15.4 \\
\hline 06JUN84 & 145 & 0 & 28.0 \\
\hline 06JUN84 & 145 & 10 & 27.3 \\
\hline 06JUN84 & 145 & 20 & 26.9 \\
\hline 06JUN84 & 145 & 30 & 26.9 \\
\hline 06JUN84 & 145 & 40 & 26.0 \\
\hline 06JUN84 & 145 & 50 & 26.1 \\
\hline 06JUN84 & 145 & 60 & 26.0 \\
\hline 06JUN84 & 145 & 70 & 25.7 \\
\hline 06JUN84 & 145 & 80 & $2 F .5$ \\
\hline 06JUN84 & 145 & 90 & 26.2 \\
\hline 06JUN84 & 145 & 100 & 26.0 \\
\hline 06JUN84 & 145 & 110 & 25.8 \\
\hline 06JUN84 & 145 & 120 & 24.7 \\
\hline 25JUL84 & 145 & 0 & 29.4 \\
\hline 25JUL84 & 145 & 10 & 27.2 \\
\hline 25JUL84 & 145 & 20 & 27.5 \\
\hline 25JUL84 & 145 & 30 & 26.8 \\
\hline 25JUL84 & 145 & 40 & 26.9 \\
\hline 25JUL84 & 145 & 50 & 26.6 \\
\hline 25JUL84 & 145 & 60 & 26.6 \\
\hline 25JUL84 & 145 & 70 & 26.8 \\
\hline 25JUL84 & 145 & 80 & 26.6 \\
\hline 25JUL84 & 145 & 90 & 26.6 \\
\hline 25JUL84 & 145 & 100 & 26.8 \\
\hline 25JUL84 & 145 & 110 & 26.4 \\
\hline 25JUL84 & 145 & 120 & 24.9 \\
\hline 09AUG84 & 145 & 0 & 31.5 \\
\hline 09AUG84 & 145 & 0 & 30.3 \\
\hline 09AUG84 & 145 & 10 & 28.3 \\
\hline 09AUG84 & 145 & 10 & 29.3 \\
\hline 09AUG84 & 145 & 20 & 28.5 \\
\hline 09AUG84 & 145 & 20 & 29.6 \\
\hline
\end{tabular}




\section{SAMPIING \\ DATE}

09AUG84

09AUG84

09AUG84

09AUG84

09AUG84

09AUG 84

09AUG84

09AUG84

O9AUG84

09AUG84

09AUG84

09AUG84

09AUG84

09AUG84

09AUG84

O9AUG84

09AUG84

09AUG84

09AUG84

O9AUG84

20AUG84

20AUG84

20AUG84

2CAUG84

20AUG84

20AUG84

20AUG84

20AUG84

20AUG8 4

20 AUG84

20AUG8 4

20AUG84

20AUG84

04SEP8 4

04SEP84

04SEP84

04SEP84

04SEP84

04SEP84

04SEP84

04SEP8 4

04SEP84

04SEP84

04SEP84

04 SEP84

$\begin{array}{cl} & \text { DISTANCE } \\ \text { DEPTH FROM } & \text { FROM } \\ \text { SURFACE }(\mathrm{cm} .) & \text { CENTER }\end{array}$

145

145

145

145

145

145

145

145

145

145

145

145

145

145

145

145

145

145

145

145

145

145

145

145

145

145

145

145

145

145

145

145

145

145

145

145

145

145

145

145

145

145

14.

145

145
30

30

40

40

50

50

60

60

70

70

80

80

90

90

100

100

110

110

120

120

0

10

20

30

40

50

60

70

80

90

100

110

120

0

10

20

30

40

50

60

70

80

90

100

110
VOLUMETRIC

MOISTURE

CONTENT ( 8 )

28.9

27.9

27.5

28.5

27.7

28.7

28.7

27.8

27.8

28.8

27.6

28.6

28.7

27.7

27.4

28.4

27.9

26.9

25.9

26.7

33.0

30.8

30.5

30.7

29.8

30.0

30.0

29.1

29.9

29.6

29.1

29.4

28.3

31.0

29.6

28.8

28.8

28.3

28.5

27.9

28.5

28.1

28.0

28.2

28.0 


SAMPLING
DATE

04SEP84

18SEP84

18 SEP 84

18SEP84

18 SEP 84

18SEP84

$18 S E P 84$

18 SEP84

$18 \mathrm{SEP} 84$

18 SEPB4

$18 \mathrm{SEP} 84$

$18 S E P 84$

18 SEP84

18 SEP84

020CT84

$020 C T 84$

020CT 84

020CT84

$020 C T 84$

02.0CT84

020CT84

020CT84

020CT 84

020CT84

020CT 84

020CT8:

020CT 24

180CT 84

$180 \mathrm{CT} 84$

180CT84

$180 C T 84$

$180 \mathrm{CT} 84$

$180 \mathrm{CT} 84$

$180 \mathrm{CT} 84$

$180 \mathrm{CT} 84$

$180 \mathrm{CT} 84$

$180 \mathrm{CT} 84$

180 TT 84

$180 \mathrm{CT} 84$

$180 \mathrm{CT} 84$

310 CT84

$310 \mathrm{CT} 84$

310СT84

$310 \mathrm{CT} 84$

310CT84

$\begin{array}{cll} & \text { DISTANCE } & \text { VOLUMETRIC } \\ \text { DEPTH FROM } & \text { FROM } & \text { MOISTURE } \\ \text { SURFACE }(\mathrm{cm} .) & \text { CENTER } & \text { CONTENT }(8)\end{array}$

145

145

145

145

145

145

145

145

145

145

145

145

145

145

145

145

145

145

145

145

145

145

145

145

145

145

145

145

145

145

145

145

145

145

145

145

145

145

145

145

145

145

145

145

145
120

0

10

20

30

40

50

60

70

80

90

100

110

120

0

10

20

30

40

50

60

70

80

90

100

110

120

0

10

20

30

40

50

60

70

80

90

100

110

120

0

10

20

30

40
26.1

28.5

27.1

26.9

26.6

26.5

26.0

26.4

26.2

26.5

26.3

26.3

25.9

24.3

27.4

26.2

25.4

25.9

25.2

25.3

25.0

25.1

24.9

24.8

24.2

24.4

22.8

28.7

26.9

27.0

26.3

26.7

26.4

25.6

26.7

26.1

26.5

26.4

25.8

24.1

27.9

26.2

26.2

26.6

25.9 


\begin{tabular}{|c|c|c|c|}
\hline & & DISTANCE & VOLUMETRIC \\
\hline SAMPLING & DEPTH FROM & FROM & MOISTURE \\
\hline DATE & SURFACE $(\mathrm{cm})$. & CENTER & CONTENT $(8)$ \\
\hline 310CT84 & 145 & 50 & 25.5 \\
\hline $310 \mathrm{CT} 84$ & 145 & 60 & 25.8 \\
\hline $310 \mathrm{CT} 84$ & 145 & 70 & 25.7 \\
\hline $310 \mathrm{CT} 84$ & 145 & 80 & 25.4 \\
\hline $310 \mathrm{CT} 84$ & 145 & 90 & 25.3 \\
\hline $310 \mathrm{CT} 84$ & 145 & 100 & 25.2 \\
\hline $310 C T 84$ & 145 & 110 & 24.7 \\
\hline $310 \mathrm{CT} 84$ & 145 & 120 & 23.3 \\
\hline 28 NOV 84 & 145 & 0 & 29.1 \\
\hline 28NOV84 & 145 & 10 & 27.8 \\
\hline 28NOV84 & 145 & 20 & 27.1 \\
\hline 28 NOV84 & 145 & 30 & 26.9 \\
\hline 28NOV84 & 145 & 40 & 27.5 \\
\hline 28NOV84 & 145 & 50 & 26.6 \\
\hline 28NOV84 & 145 & 60 & 26.6 \\
\hline 28 NOV84 & 145 & 70 & 26.3 \\
\hline 28NOV84 & 145 & 80 & 26.7 \\
\hline 28NOV84 & 145 & 90 & 26.8 \\
\hline 28NOV84 & 145 & 100 & 26.4 \\
\hline 28NOV84 & 145 & 110 & 26.2 \\
\hline 28 NOV 84 & 145 & 120 & 25.2 \\
\hline 03JAN85 & 145 & 0 & 28.2 \\
\hline 03JAN85 & 145 & 60 & 26.3 \\
\hline 03JAN85 & 145 & 120 & 25.0 \\
\hline 17JAN85 & 145 & 0 & 30.6 \\
\hline 17JAN85 & 145 & 60 & 28.0 \\
\hline 17JAN85 & 145 & 120 & 26.6 \\
\hline 04FEB85 & 145 & 0 & 27.7 \\
\hline $04 F E B 85$ & 145 & 60 & 24.7 \\
\hline 04FEB85 & 145 & 120 & 22.6 \\
\hline 20FEB 85 & 145 & 0 & 28.7 \\
\hline 20FEB85 & 145 & 60 & 27.0 \\
\hline 20FEB 85 & 145 & 120 & 25.7 \\
\hline 06MAR85 & 145 & 0 & 30.2 \\
\hline 06MAR85 & 145 & 60 & 27.3 \\
\hline 06MAR85 & 145 & 120 & 26.1 \\
\hline 21MAR85 & 145 & 0 & 30.5 \\
\hline 21MAR85 & 145 & 60 & 28.2 \\
\hline 21MAR85 & 145 & 120 & 26.5 \\
\hline 01APR85 & 145 & 0 & 32.0 \\
\hline 01APR85 & 145 & 60 & 29.1 \\
\hline 01APR8 5 & 145 & 120 & 27.9 \\
\hline 08APR85 & 145 & 0 & 30.3 \\
\hline 08APR85 & 145 & 60 & 27.8 \\
\hline 08APR8 5 & 145 & 120 & 25.9 \\
\hline
\end{tabular}




\section{SAMPLING \\ DATE}

25APR85
25APR 85
2 5APR85
29APR85
29APR85

29APR 85

30APR 85

30APR 85

$30 A P R 85$

01MAY 85

01MAY85

01MAY85

02 MAY85

02MAY85

02MAY85

$03 M A Y 85$

03MAY85

03 MAY85

06MAY85

06MAY85

06MAY85

O8MAY85

08MAY85

08MAY85

10MAY85

10MAY85

10MAY85

13MAY85

13 MAY85

13MAY85

15MAY85

15MAY85

$15 \mathrm{MAY} 85$

17MAY85

17MAY85

17MAY85

21MAY85

21MAY85

21MAY85

23MAY85

23 MAY85

23 MAY 85

28MAY85

28MAY85

28MAY85

$\begin{array}{cll} & \text { DISTANCE } & \text { VOLUMETRIC } \\ \text { DEPTH FROM } & \text { FROM } & \text { MOISTURE } \\ \text { SURFACE (cm.) } & \text { CENTER } & \text { CONTENT }(8)\end{array}$

145

145

145

145

145

145

145

145

145

145

145

145

145

145

145

145

145

145

145

145

145

145

145

145

145

145

145

145

145

145

145

145

145

145

145

145

145

145

145

145

145

145

145

145

145
28.8

26.7

24.7

29.3

27.0

25.7

28.5

26.3

24.2

27.6

25.1

22.8

27.0

24.1

18.9

26.5

23.8

19.0

25.4

22.4

19.8

24.0

21.7

17.7

23.5

21.4

17.6

22.8

21.0

18.4

22.0

21.2

17.5

22.0

20.6

18.1

21.6

20.5

17.5

21.4

20.2

17.7

21.0

19.8

16.8 


\begin{tabular}{|c|c|c|c|}
\hline $\begin{array}{l}\text { SAMPLING } \\
\text { DATE }\end{array}$ & $\begin{array}{l}\text { DEPTH FROM } \\
\text { SURFACE }(\mathrm{cm} .)\end{array}$ & $\begin{array}{l}\text { DISTANCE } \\
\text { FROM } \\
\text { CENTER }\end{array}$ & $\begin{array}{l}\text { VOLUMETR } \\
\text { MOISTURE } \\
\text { CONTENT }\end{array}$ \\
\hline 30MAY85 & 145 & 0 & 20.8 \\
\hline 30MAI 85 & 145 & 60 & 19.6 \\
\hline 30MAY85 & 145 & 120 & 16.6 \\
\hline 04JUN85 & 145 & 0 & 21.0 \\
\hline 04JUN85 & 145 & 60 & 19.7 \\
\hline 04.JUN85 & 145 & 120 & 15.9 \\
\hline 12JUN85 & 145 & 0 & 19.9 \\
\hline 12JUN8 5 & 145 & 60 & 19.3 \\
\hline 12JUN85 & 145 & 120 & 16.1 \\
\hline 18JUN85 & 145 & 0 & 20.1 \\
\hline 18JUN85 & 145 & 60 & 18.8 \\
\hline 18JUN85 & 145 & 120 & 15.4 \\
\hline 25JUN85 & 145 & 0 & 19.5 \\
\hline 25JUN85 & 145 & 60 & 18.9 \\
\hline 25JUN85 & 145 & 120 & 15.7 \\
\hline 02JUL85 & 145 & 0 & 19.3 \\
\hline 02JUL85 & 145. & 60 & 18.4 \\
\hline 02JUL85 & 145 & 120 & 14.3 \\
\hline 11JUL85 & 145 & 0 & 18.7 \\
\hline 11JUL85 & 145 & 60 & 18.0 \\
\hline 11JUL85 & 145 & 120 & 15.0 \\
\hline 12JUL85 & 145 & 0 & 18.6 \\
\hline 12JUL85 & 145 & 60 & 17.5 \\
\hline 12JUL85 & 145 & 120 & 14.5 \\
\hline 13JUL85 & 145 & 0 & 18.8 \\
\hline 13JUL85 & 145 & 60 & 17.8 \\
\hline 13JUL85 & 145 & 120 & 14.1 \\
\hline 14JUL85 & 145 & 0 & 21.0 \\
\hline 14JUL85 & 145 & 60 & 19.4 \\
\hline 14JUL85 & 145 & 120 & 16.7 \\
\hline 15JUL85 & 145 & 0 & 26.6 \\
\hline 15JUL85 & 145 & 60 & 24.0 \\
\hline 15JUL85 & 145 & 120 & 18.7 \\
\hline 16JUL85 & 145 & 0 & 27.2 \\
\hline 16JUL85 & 145 & 60 & 25.2 \\
\hline 16JUL85 & 145 & $? 20$ & 19.4 \\
\hline 17JUL85 & 145 & 0 & 27.5 \\
\hline 17JUL85 & 145 & 60 & 25.5 \\
\hline 17JUL85 & 145 & 120 & 22.6 \\
\hline 18JUL85 & 145 & 0 & 27.2 \\
\hline 18JUL85 & 145 & 60 & 25.0 \\
\hline 18JUL85 & 145 & 120 & 22.1 \\
\hline 19JUL85 & 145 & 0 & 26.9 \\
\hline 19JUL85 & 145 & 60 & 25.2 \\
\hline 19JUL85 & 145 & 120 & 19.7 \\
\hline
\end{tabular}




\section{SAMPLING \\ DATE}

21JUL85

21JUL85

21JUL85

22JUL85

22JUL85

22JUL85

23JUL85

23JUL85

23JUL.85

24JUL85

24JUL85

24JUL8 5

25JUL85

25JUL85

25JUL85

26JUL85

26JUL85

26JUL85

27JUL85

27JUL85

27JUL85

28JUL85

28JUL85

28JUL85

28 JUL85

28JUL85

28JUL85

29JUL85

29JUL85

29JUL85

30 JUL 85

30 JUL 85

30 JUL85

02AUG85

02AUG85

02 AUG85

04AUG85

04AUG85

04AUG85

06AUG 85

06AUG8 5

06AUG8 5

09AUG85

09AUG85

09AUG8 5
DEPTH FROM

SURFACE $(\mathrm{cm}$.

145

145

145

145

145

145

145

145

145

145

145

145

145

145

145

145

145

145

145

145

145

145

145

145

145

145

145

145

145

145

145

145

145

145

145

145

145

145

145

145

145

145

145

145

145 $\begin{array}{ll}\text { DISTANCE } & \text { VOLUMETRIC } \\ \text { FROM } & \text { MOISTURE } \\ \text { CENTER } & \text { CONTENT (z) }\end{array}$

27.9

25.0

22.1

27.4

25.2

22.7

27.4

25.2

22.6

28.2

25.4

22.3

27.7

25.4

21.9

28.1

25.7

21.7

27.7

25.3

24.3

29.0

28.1

25.8

26.6

23.4

24.6

27.9

25.3

23.0

28.1

25.4

22.6

27.4

25.6

21.7

27.8

25.7

23.7

28.9

26.1

23.6

28.0

25.8

24.2 
-TREAT $=145 \mathrm{~cm}$. HORIZONTAL TUBE TUBE NUMBER=803

SAMPLING
DATE

14AUG85

1.4AUG 85

14 AUG 85

21 AUG 85

21AUG85

21 AUG 85

23AUG85

23 AUG85

23 AUG85

27 AUG 85

27 AUG85

27 AUG 85

30 AUG 85

30 AUG 85

$30 A U G G 85$

03SEP 85

$03 S E P 85$

O3SEP85

09S 85

09SiY J

09SEP85

18 SEP 85

18SEP 85

18SEP 85

$30 S E P 85$

3 USEP 85

30SEP8 5

$090 \mathrm{CT} 85$

$090 C T 85$

$090 \mathrm{CT} 85$

$160 \mathrm{CT} 85$

$160 \mathrm{CT} 85$

$160 \mathrm{CT} 85$

230 CT 85

$230 \mathrm{CT} 85$

$230 \mathrm{CT} 85$

300 CT 85

300CT 85

300CT 85

06NOV85

06NOV85

06NOV85

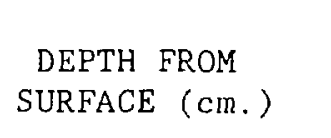

145

145

145

145

145

145

145

145

145

145

145

14.5

145

145

145

145

145

145

145

145

145

145

145

145

145

145

145

145

145

145

145

145

145

145

145

145

145

145

145

145

145

145

$\begin{array}{ll}\text { DISTANCE } & \text { VOLUMETRIC } \\ \text { FROM } & \text { MOISTURE } \\ \text { CENTER } & \text { CONTENT }(8)\end{array}$

28.4

26.1

23.9

28.8

26.3

23.8

28.5

25.9

24.3

28.3

25.8

24.6

28.6

25.6

24.1

28.7

25.8

24.3

28.5

25.9

24.2

28.6

26.3

24.7

28.2

25.9

24.0

28.3

26.2

22.6

28.8

25.4

24.4

29.1

25.6

24.4

28.5

25.8

24.4

27.7

26.0

22.9 


\section{SAMPLING \\ DATE}

12APR 84

12APR 84

12APR 84

12APR 84

12APR 84

12APR 84

12APR 84

12 APR 84

12 APR 84

12APR 84

12APR84

12APR84

12APR 84

12APR 84

10MAY84

10MAY84

10MAY84

1DNAY84

10MAY84

10MAY84

10MAY84

10MAY84

10MAY84

10MAY84

10 MAY84

10MAY84

10MAY84

06JUN84

06JUN84

06JUN84

06JUN84

06JUN84

06JUN84

06JUN84

06JUN84

06JUN84

C5JUN84

06JUN84

06JUN84

06JUN84

25JUL84

25JUL84

25JUL84

25JUL 84

25JUL84
DEPTH FROM

SURFACE (cm.)

220

220

220

220

220

220

220

220

220

220

220

220

220

220

220

220

220

220

220

220

220

220

220

220

220

220

220

220

220

220

220

220

220

220

220

220

220

220

220

220

220

220

220

220

220
DISTANCE VOLUMETRIC

FROM MOISTURE

CENTER

CONTENT ( 8 )

10
20
30
40
50
60
70

80

90

100

110

120

130

0

10

20

30

40

50

60

70

80

90

100

110

120

0

10

20

30

40

50

60

70

80

90

100

110

120

0

10

20

30

40
21.6

20.2

20.6

20.3

20.0

20.3

20.1

20.4

19.8

19.9

19.4

18.9

17.1

12.5

19.8

19.0

19.0

18.8

18.6

18.9

18.9

18.6

18.3

18.3

18.0

17.2

16.1

28.3

27.1

26.2

26.3

26.1

26.1

25.9

26.0

26.0

26.1

25.8

25.4

24.2

29.1

27.8

27.3

27.2

27.1 


\begin{tabular}{|c|c|c|c|}
\hline $\begin{array}{l}\text { SAMPLING } \\
\text { DATE }\end{array}$ & $\begin{array}{l}\text { DEPTH FROM } \\
\text { SURFACE (cm.) }\end{array}$ & $\begin{array}{l}\text { DISTANCE } \\
\text { FROM } \\
\text { CENTER }\end{array}$ & $\begin{array}{l}\text { VOLUMETRIC } \\
\text { MOISTURE } \\
\text { CONTENT }(8))\end{array}$ \\
\hline 25JUL84 & 220 & 50 & 26.6 \\
\hline 25JUL84 & 220 & 60 & 26.8 \\
\hline 25JUL84 & 220 & 70 & 26.6 \\
\hline 25JUL84 & 220 & 80 & 26.9 \\
\hline 2.5JUL84 & 220 & 90 & 27.4 \\
\hline 25JUL84 & 220 & 100 & 26.9 \\
\hline 25JUL84 & 220 & 110 & 26.7 \\
\hline 25JUL84 & 220 & 120 & 25.6 \\
\hline 09AUG84 & 220 & 0 & 31.1 \\
\hline 09AUG84 & 220 & 0 & 30.0 \\
\hline 09AUG84 & 220 & 10 & 28.7 \\
\hline 09AUG84 & 220 & 10 & 29.7 \\
\hline 09AUG84 & 220 & 20 & 29.8 \\
\hline 09AUG84 & 220 & 20 & 28.8 \\
\hline 09AUG84 & 220 & 30 & 28.5 \\
\hline 09AUG84 & 220 & 30 & 29.5 \\
\hline 09AUG84 & 220 & 40 & 29.3 \\
\hline 09.AUG84 & 220 & 40 & 28.2 \\
\hline 09AUG84 & 220 & 50 & 27.9 \\
\hline 09AUG84 & 220 & 50 & 28.8 \\
\hline 09AUG84 & 220 & 60 & 27.8 \\
\hline 09AUG84 & 220 & 60 & 28.8 \\
\hline 09AUG84 & 220 & 70 & 28.8 \\
\hline 09AUG84 & 220 & 70 & 27.8 \\
\hline 09AUG84 & 220 & 80 & 28.1 \\
\hline 09AUG84 & 220 & 80 & 29.1 \\
\hline 09AUG84 & 220 & 90 & 28.0 \\
\hline 09AUG84 & 220 & 90 & 29.0 \\
\hline 09AUG84 & 220 & 100 & 29.3 \\
\hline 09AUG84 & 220 & 100 & 28.2 \\
\hline 09AUG84 & 220 & 110 & 27.5 \\
\hline 09AUG84 & 220 & 110 & 28.5 \\
\hline 09AUG84 & 220 & 120 & 27.5 \\
\hline 09AUG84 & 220 & 120 & 26.6 \\
\hline 20AUG84 & 220 & 0 & 32.9 \\
\hline 20AUG84 & 220 & 10 & 31.2 \\
\hline 20AUG84. & 220 & 20 & 30.6 \\
\hline 20AUG84 & 220 & 30 & 29.9 \\
\hline 20AUG84 & 220 & 40 & 29.8 \\
\hline 20AUG84 & 220 & 50 & 29.9 \\
\hline 20AUG84 & 220 & 60 & 29.2 \\
\hline 20AUG84 & 220 & 70 & 29.8 \\
\hline 20AIJG84 & 220 & 80 & 30.1 \\
\hline 20AUG84 & 220 & 90 & 30.4 \\
\hline 20AUG84 & 220 & 100 & 29.8 \\
\hline
\end{tabular}




\begin{tabular}{|c|c|c|c|}
\hline $\begin{array}{l}\text { SAMPLING } \\
\text { DATE }\end{array}$ & $\begin{array}{c}\text { DEPTH FROM } \\
\text { SURFACE }(\mathrm{cm} .)\end{array}$ & $\begin{array}{l}\text { DISTANCE } \\
\text { FROM } \\
\text { CENTER }\end{array}$ & $\begin{array}{l}\text { VOLUMETRIC } \\
\text { MOISTURE } \\
\text { CONTENT }(\gamma)\end{array}$ \\
\hline 20AUG84 & 220 & 110 & 29.7 \\
\hline 20AUG84 & 220 & 120 & 28.2 \\
\hline 04SEP8/ & 220 & 0 & 31.5 \\
\hline 04SEP84 & 220 & 10 & 29.7 \\
\hline 04SEP84 & 220 & 20 & 29.1 \\
\hline 04SEP84 & 220 & 30 & 28.7 \\
\hline 04SEP84 & 220 & 40 & 28.7 \\
\hline 04SEP84 & 220 & 50 & 27.8 \\
\hline 04SEP84 & 220 & 60 & 28.3 \\
\hline 04SEP84 & 220 & 70 & 28.5 \\
\hline 04SEP84 & 220 & 80 & 29.1 \\
\hline 04SEP84 & 220 & 90 & 28.8 \\
\hline 04SEP84 & 220 & 100 & 28.6 \\
\hline 04SEP84 & 220 & 110 & 28.3 \\
\hline 04SEP84 & 220 & 120 & 26.3 \\
\hline 18SEP84 & 220 & 0 & 29.1 \\
\hline 18SEP84 & 220 & 10 & 27.2 \\
\hline 18SEP84 & 220 & 20 & 26.9 \\
\hline 18SEP84 & 220 & 30 & 26.4 \\
\hline 18SEP84 & 220 & 40 & 26.0 \\
\hline 18SEP84 & 220 & 50 & 26.0 \\
\hline 18 SEP 84 & 220 & 60 & 26.1 \\
\hline 18SEP84 & 220 & 70 & 26.1 \\
\hline 18SEP84 & 220 & 80 & 26.4 \\
\hline 18SEP84 & 220 & 90 & 26.6 \\
\hline 18SEP84 & 220 & 100 & 26.3 \\
\hline 18SEP84 & 220 & 110 & 26.2 \\
\hline 18SEP84 & 220 & 120 & 25.2 \\
\hline 020CT84 & 220 & 0 & 27.4 \\
\hline 020CT84 & 220 & 10 & 25.2 \\
\hline 020CT84 & 220 & 20 & 24.8 \\
\hline 020CT84 & 220 & 30 & 24.9 \\
\hline 020CT84 & 220 & 40 & 24.6 \\
\hline 020CT84 & 220 & 50 & 24.7 \\
\hline 020CT84 & 220 & 60 & 25.0 \\
\hline 020CT84 & 220 & 70 & 24.7 \\
\hline 020CT 84 & 220 & 80 & 24.7 \\
\hline $020 \mathrm{CT} 84$ & 220 & 90 & 24.4 \\
\hline 020CT84 & 220 & 100 & 24.2 \\
\hline $020 \mathrm{CT} 84$ & 220 & 110 & 24.3 \\
\hline 020CT84 & 220 & 120 & 22.3 \\
\hline $180 \mathrm{CT} 84$ & 220 & 0 & 28.4 \\
\hline $180 \mathrm{CT} 84$ & 220 & 10 & 27.2 \\
\hline $180 \mathrm{CT} 84$ & 220 & 20 & 26.9 \\
\hline $180 \mathrm{CT} 84$ & 220 & 30 & 26.7 \\
\hline
\end{tabular}




\begin{tabular}{|c|c|c|c|}
\hline SAMPLING & DEPTH FROM & $\begin{array}{l}\text { DISTANCE } \\
\text { FROM }\end{array}$ & $\begin{array}{l}\text { VOLUMETRIC } \\
\text { MOISTURE }\end{array}$ \\
\hline DATE & SURFACE $(\mathrm{cm})$. & CENTER & CONTENT $(q)$ \\
\hline $180 \mathrm{CT} 84$ & 220 & 40 & 26.8 \\
\hline $180 \mathrm{CT} 84$ & 220 & 50 & 26.4 \\
\hline $180 \mathrm{CT} 84$ & 220 & 60 & 25.4 \\
\hline $180 \mathrm{CT} 84$ & 220 & 70 & 26.3 \\
\hline $180 \mathrm{CT} 84$ & 220 & 80 & 26.2 \\
\hline $180 \mathrm{CT} 84$ & 220 & 90 & 25.6 \\
\hline $180 \mathrm{CT} 84$ & 220 & 100 & 26.2 \\
\hline $180 \mathrm{CT} 84$ & 220 & 110 & 26.1 \\
\hline $180 \mathrm{CT} 84$ & 220 & 120 & 25.0 \\
\hline $310 \mathrm{CT} 84$ & 220 & 0 & 27.9 \\
\hline $310 \mathrm{CT} 84$ & 220 & 10 & 26.7 \\
\hline $310 \mathrm{CT} 84$ & 220 & 20 & 26.0 \\
\hline $310 \mathrm{CT} 84$ & 220 & 30 & 25.9 \\
\hline $310 \mathrm{CT} 84$ & 220 & 40 & 26.0 \\
\hline $310 \mathrm{CT} 84$ & 220 & 50 & 25.9 \\
\hline $310 \mathrm{CT} 84$ & 220 & 60 & 25.6 \\
\hline $310 \mathrm{CT} 84$ & 220 & 70 & 25.7 \\
\hline $310 \mathrm{CT} 84$ & 220 & 80 & 25.8 \\
\hline $310 \mathrm{CT} 84$ & 220 & 90 & 25.8 \\
\hline $310 \mathrm{CT} 84$ & 220 & 100 & 25.5 \\
\hline $310 \mathrm{CT} 84$ & 220 & 110 & 25.3 \\
\hline $310 \mathrm{CT} 84$ & 220 & 120 & 24.1 \\
\hline 28 NOV 84 & 220 & 0 & 29.5 \\
\hline 28 NOV84 & 220 & 10 & 27.9 \\
\hline 28NOV84 & 220 & 20 & 27.0 \\
\hline 28 NOV 84 & 220 & 30 & 26.8 \\
\hline 28 NOV84 & 220 & 40 & 27.0 \\
\hline 28 NOV84 & 220 & 50 & 26.7 \\
\hline 28NOV84 & 220 & 60 & 26.8 \\
\hline 28NOV84 & 220 & 70 & 26.9 \\
\hline 28 NOV 84 & 220 & 80 & 26.9 \\
\hline 28NOV84 & 220 & 90 & 27.2 \\
\hline 28 NOV 84 & 220 & 100 & 27.0 \\
\hline 28 NOV84 & 220 & 110 & 26.6 \\
\hline 28NOV84 & 220 & 120 & 25.2 \\
\hline 03JAN85 & 220 & 0 & 28.6 \\
\hline 03JAN85 & 220 & 60 & 26.4 \\
\hline 03JAN85 & 220 & 120 & 25.0 \\
\hline 17JAN85 & 220 & 0 & 30.5 \\
\hline 17JAN85 & 220 & 60 & 28.5 \\
\hline 17JAN85 & 220 & 120 & 27.1 \\
\hline 04FEB85 & 220 & 0 & 28.3 \\
\hline 04FEB85 & 220 & 60 & 25.1 \\
\hline 04FE'B85 & 220 & 120 & 23.6 \\
\hline 20FEB85 & 220 & 0 & 29.7 \\
\hline
\end{tabular}




\section{SAMPLING DATE}

\section{FEB 85}

20FEB 85

06MAR85

06MAR85

O6MAR 85

21MAR 85

21MAR85

21MAR 85

01APR 85

01APR85

01APR 85

08APR8 5

08APR 5

08APR 85

25APR 85

25APR 85

25APR 85

29APR85

29APR 85

29APR 85

30APR85

30APR 85

30APR 85

O1MAY85

01MAY85

01MAY85

02 MAY85

02MAY85

02 MAY85

$03 M A Y 85$

03MAY85

03 MAY 85

06MAY85

06MAY85

06MAY85

$08 M A Y 85$

OBMAY 85

08MAY 85

10MAY85

10MAY85

10MAY85

13 MAY85

13MAY85

13 MAY85

15 MAY 85
DEPTH FROM

SURFACE (cm.)

220

220

220

220

220

220

220

220

220

220

220

220

220

220

220

220

220

220

220

220

220

220

220

220

220

220

220

220

220

220 ,

220

220

220

220

220

220

220

220

220

220

220

220

220

220

220
DISTANCE VOLUMETRIC FROM MOISTURE

CENTER

CONTENT (\&)

27.4

26.3

31.2

27.6

26.5

31.3

28.0

26.5

32.0

30.1

28.2

31.0

27. 8

26.0

29.5

26.5

25.4

29.5

27.2

26.0

29.6

26.8

24.2

28.3

25.9

23.9

27.4

25.5

21.1

27.1

24.8

21.8

26.1

23.8

21.6

24.8

23.0

18.6

24.5

22.8

19.2

23.8

22.0

19.4

23.3 


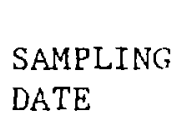

15MAY85

15MAY85

17 MAY85

17 MAY85

17 MAY 85

2.1MAY85

21MAY85

21 MAY 85

23MAY85

23 MAY85

23 MAY 85

28MAY 85

28MAY 85

$28 M A Y 85$

30MAY 85

30MAY85

30MAY85

04JUN8 5

04JUN85

04JUN85

12 JUN85

12 JUN85

12JUN85

18JUN85

18 JUN85

18JUN85

25JUN85

25 JUN 85

25JUN85

02JUL85

02JUL85

02JUL85

11 JUL85

11 JUL 85

11JUL85

12JUL85

12JUL85

12JUL85

13JUL85

13 JUL85

13JUL85

14JUL85

14JUL8 5

14JUL85

15 JUL8 5
DEPTH FROM

SURFACE (cm.)

220

220

220

220

220

220

220

220

22.0

220

220

220

220

220

220

220

220

220

220

220

220

220

220

220

220

220

220

220

220

220

220

220

220

220

220

220

220

220

220

220

220

220

220

220

220
DISTANCE VOLUMETRIC

FROM

CEN'TER

60

120

0

60

120

0

60

120

0

60

120

0

60

120

0

60

120

0

60

120

0

60

120

0

60

120

0

60

120

0

60

120

0

60

120

0

60

120

0

60

120

0

60

120

0
MOISTURE

CONTENT (8)

21.7

19.0

22. 8

21.3

19.4

22.3

21.3

18.1

22.3

20.6

17.5

22.0

20.3

17.9

21.9

20.6

18.0

21.8

20.2

17.5

21.0

19.4

16.6

20.9

19.9

16.2

20.4

19.1

16.3

19.6

18.7

15.7

19.8

18.6

15.7

19.1

18.2

15.3

18.8

17.7

15.0

19.4

17.9

15.0

19.4 


\begin{tabular}{|c|c|c|c|}
\hline $\begin{array}{l}\text { SAMPLING } \\
\text { DATE }\end{array}$ & $\begin{array}{l}\text { DEPTH FROM } \\
\text { SURFACE (cm.) }\end{array}$ & $\begin{array}{l}\text { DISTANCE } \\
\text { FROM } \\
\text { CENTER }\end{array}$ & $\begin{array}{l}\text { VOLUMETR } \\
\text { MOISTURE } \\
\text { CONTENT }\end{array}$ \\
\hline 15JUL85 & 220 & 60 & 18.4 \\
\hline 15JUL85 & 220 & 120 & 11.2 \\
\hline 16JUL85 & 220 & 0 & 20.5 \\
\hline 16JUL85 & 220 & 60 & 19.4 \\
\hline 16JUL85 & 220 & 120 & 12.7 \\
\hline $17 \mathrm{JUL} 85$ & 220 & 0 & 26.0 \\
\hline 17JUL85 & 220 & 60 & 23.2 \\
\hline 17JUL85 & 220 & 120 & 21.7 \\
\hline 18JUL85 & 220 & 0 & 28.2 \\
\hline 18JUL85 & 220 & 60 & 25.4 \\
\hline 18JUL85 & 220 & 120 & 23.4 \\
\hline 19JUL85 & 220 & 0 & 28.0 \\
\hline 19JUL85 & 220 & 60 & 25.4 \\
\hline 19JUL85 & 220 & 120 & 20.5 \\
\hline 21JUL85 & 220 & 0 & 27.9 \\
\hline 21JUL85 & 220 & 60 & 25.6 \\
\hline 21JUL85 & 220 & 120 & 23.2 \\
\hline 22JUL85 & 220 & 0 & 28.0 \\
\hline 22JUL85 & 220 & 60 & 25.4 \\
\hline 22JUL85 & 220 & 120 & 22.9 \\
\hline 23JUL85 & 220 & 0 & 28.0 \\
\hline 23JUL85 & 220 & 60 & 25.1 \\
\hline 23JUL85 & 220 & 120 & 23.6 \\
\hline 24JUL85 & 220 & 0 & 28.0 \\
\hline 24JUL85 & 220 & 60 & 25.9 \\
\hline 24JUL85 & 220 & 120 & 22.9 \\
\hline 25JUI_85 & 220 & 0 & 27.9 \\
\hline 25JUL85 & 220 & 60 & 25.2 \\
\hline 25JUL85 & 220 & 120 & 21.9 \\
\hline 26JUL85 & 220 & 0 & 28.4 \\
\hline 26JUL85 & 220 & 60 & 25.6 \\
\hline 26JUL85 & 220 & 120 & 23.4 \\
\hline 27JUL85 & 220 & 0 & 28.0 \\
\hline 27JUL85 & 220 & 60 & 25.9 \\
\hline 27JUL85 & 220 & 120 & 23.9 \\
\hline 28JUL85 & 220 & 0 & 28.2 \\
\hline 28JUL85 & 220 & 60 & 25.8 \\
\hline 28JUL85 & 220 & 120 & 24.0 \\
\hline 29JUL85 & 220 & 0 & 27.9 \\
\hline 29JUL85 & 220 & 60 & 25.6 \\
\hline 29JUL85 & 220 & 120 & 23.6 \\
\hline 30JUL85 & 220 & 0 & 28.1 \\
\hline 30JUL85 & 220 & 60 & 26.2 \\
\hline 30J JL85 & 220 & 120 & 23.6 \\
\hline 02AUG85 & 220 & 0 & 28.6 \\
\hline
\end{tabular}




SAMPLING
DATE

$02 A U G 85$

$02 A U G 85$

04AUG85

04 AUG8 5

04AUG85

06AUG85

06AUG85

06AUG85

09AUG85

09AUG85

09AUG85

14 AUG85

14 AUG85

14 AUG85

16 AUG85

16AUG85

16AUG85

21AUG85

21AUG85

21AUG85

23AUG85

23AUG85

23AUG85

27 AUG85

27 AUG 85

27 AUG 5

30AUG85

30 AUG 85

30AUG 8

$03 S E P 85$

03SEP85

03SEP 85

09SEP 85

09SEP 85

09SEP85

18SEP8 5

18 SEP 85

18SEP85

30 SEP 85

$30 S E P 85$

30 SEP85

$090 \mathrm{CT} 85$

090CT8 5

$090 \mathrm{CT} 85$

160CT85
DEPTH FROM

SURFACE (cm.)

220

220

220

220

220

220

220

220

220

220

220

220

220

220

220

220

220

220

220

220

220

220

220

220

220

220

220

220

220

220

220

220

220

220

220

220

220

220

220

220

220

220

220

220

220

$\begin{array}{ll}\text { DISTANCE } & \text { VOLUMETRIC } \\ \text { FROM } & \text { MOISTURE } \\ \text { CENTER } & \text { CONTENT }(q)\end{array}$

26.2

22.9

28.7

25.8

24.3

29.0

25.8

24.6

28.5

25.9

24.3

28.7

26.1

24.1

28.6

26.4

24.5

28.6

26.1

24.4

29.1

26.1

24.5

28.8

26.3

24.1

28.5

26.0

24.5

28.7

26.6

24.5

28.4

26.4

24.6

28.9

26.4

24.7

28.9

25.9

24.7

29.0

26.4

23.3

28.4 
SAMPLING
DATE

$160 \mathrm{CT} 85$

$160 \mathrm{CT} 85$

230 CT 85

$230 \mathrm{CT} 85$

230CT 85

$300 C T 85$

300CT 85

$300 \mathrm{CT} 85$

06NOV85

06NOV85

06NOV85

DEPTH FROM
SURFACE (cm.)

220

220

220

220

220

220

220

220

220

220

220

$\begin{array}{ll}\text { DISTANCE } & \text { VOLUMETRIC } \\ \text { FROM } & \text { MOISTURE } \\ \text { CENTER } & \text { CONTENT }(8)\end{array}$

60

27.0

120

0

60

120

0

60

120

0

60

120
24.8

29.0

25.9

24.4

28.2

25.9

23.3

28.3

27.0

23.4 
SAMPLING
DATE

12APR 84

12APR 84

12APR 84

12APR 84

12APR84

12APR84

12APR84

12APR84

12APR84

12APR84

12APR 84

12APR 84

12APR 84

12APR 84

10MAY84

10MAY84

10MAY84

1OMAY 84

10MAY 84

10MAY84

10MAY84

10MAY 84

10MAY 84

10MAY84

10MAY 84

10MAY84

10MAY84

06JUN84

06JUN84

06JUN84

06JUN84

06JUN84

06JUN84

06JUN84

06JUN84

06JUN84

06JUN84

06JUN84

06JUN84

06JUN84

25JUL84

25 JUL84

25JUL84

25JUL84

25JUL84

DEPTH FROM
SURFACE (cm.)

295

295

295

295

295

295

295

295

295

295

295

295

295

295

295

295

295

295

295

295

295

295

295

295

295

295

295

295

295

295

295

295

295

295

295

295

295

295

295

295

295

295

295

295

295
DISTANCE VOLUMETRIC

FROM

CENTER

MOISTURE

CONTENT ( 8 )

21.8

21.2

20.4

20.3

20.5

20.7

21.3

20.8

20.6

20.4

19.8

19.1

17.7

12.4

20.2

19.6

19.5

19.3

19.3

19.4

19.5

19.4

19.3

19.0

18.4

18.0

16.5

28.5

26.8

26.6

26.7

26.5

26.6

26.2

26.5

26.5

25.8

26.0

25.7

24.5

28.9

27.5

27.1

26.9

27.2 
SAMPLING

DATE

25JUL84

25JUL84

25JUL84

25JUL84

25JUL84

25JUL84

25JUL84

25JUL84

09AUG84

09AUG84

09AUG84

09AUG84

09AUG84

09AUG84

09AUG84

09AUG84

09AUG84

09AUG84

$09 A U G 84$

$09 A U G 84$

09AUG84

09AUG84

09AUG84

09AUG84

09AUG84

09AUG84

09AUG84

09AUG84

09AUG84

09AUG84

09AUG84

09AUG84

09AUG84

09AUG84

20AUG84

20AUG84

20AUG84

20AUG84

20AUG84

20AUG84

20AUG84

20AUG84

20AUG84

20AUG84

20AUG84
DEPTH FROM

SURFACE $(\mathrm{cm}$.

295

295

295

295

295

295

295

295

295

295

295

295

295

295

295

295

295

295

295

295

295

295

295

295

295

295

295

295

295

295

295

295

295

295

295

295

295

295

295

295

295

295

295

295

295
DISTANCE VOLUMETRIC

FROM MOISTURE

CENTER

CONTENT

(8)

$\begin{array}{rr}50 & 27.1 \\ 60 & 27.2 \\ 70 & 26.9 \\ 80 & 26.9 \\ 90 & 26.9 \\ 100 & 26.7 \\ 110 & 27.4 \\ 120 & 26.0 \\ 0 & 30.9 \\ 0 & 29.8 \\ 10 & 30.2 \\ 10 & 29.1 \\ 20 & 29.8 \\ 20 & 28.8 \\ 30 & 29.4 \\ 30 & 28.4 \\ 40 & 28.8\end{array}$

$40 \quad 28.8$

$40 \quad 29.9$

$50 \quad 29.3$

$50 \quad 30.3$

$60 \quad 29.1$

$60 \quad 28.1$

$70 \quad 29.0$

$70 \quad 28.0$

$80 \quad 29.2$

$80 \quad 28.2$

$90 \quad 29.2$

$90 \quad 28.2$

$100 \quad 29.0$

100

110

30.1

29.4

110

120

28.4

27.6

120

28.6

31.9

30.0

30.6

30.9

30.8

30.8

30.6

30.5

30.3

29.6

29.8 


SAMPLING
DATE

20AUG84

20AUG84

04SEP84

04SEP84

04SEP84

04SEP 84

04SEP84

04 SEP8 4

04SEP84

04SEP84

04SEP84

04SEP84

04SEP84

04SEP84

04SEP84

18SEP84

18SEP84

18SEP 84

18SEP84

$18 S E P 84$

$18 S E P 84$

$185 \mathrm{SPP} 84$

$18 S E P 84$

$18 S E P 84$

$18 S E P 84$

$18 \mathrm{SEP} 84$

18SEP84

18SEP84

020CT 84

020CT 84

020CT 84

020CT84

020CT 84

020CT 84

020CT84

$020 \mathrm{CT} 84$

$020 \mathrm{CT} 84$

020CT84

020CT 84

020CT84

$020 C T 84$

$180 \mathrm{CT} 84$

$180 \mathrm{CT} 84$

180CT 84

180CT 84

$\begin{array}{cll} & \text { DISTANCE } & \text { VOLUMETRIC } \\ \text { DEPTH FROM } & \text { FROM } & \text { MOISTURE } \\ \text { SURFACE (cm.) } & \text { CENTER } & \text { CONTENT }(q)\end{array}$

295

295

295

295

295

295

295

295

295

295

295

295

295

295

295

295

295

295

295

295

295

295

295

295

295

295

295

295

295

295

295

295

295

295

295

295

295

295

295

295

295

295

295

295

295
110

120

0

10

20

30

40

50

60

70

80

90

100

110

120

0

10

20

30

40

50

60

70

80

90

100

110

120

0

10

20

30

40

50

60

70

80

90

100

110

120

0

10

20

30
30.3

29.2

30.2

29.8

29.4

29.2

29.5

29.7

29.2

29.3

29.3

29.2

29.2

29.2

28.1

28.7

26.9

26.9

27.4

27.1

27.0

26.8

26.8

27.0

26.8

27.2

26.9

25.5

27.2

25.9

25.9

25.7

25.8

25.9

26.2

25.8

25.2

24.7

25.0

24.9

23.7

28.9

26.6

27.2

26.9 


\begin{tabular}{|c|c|c|c|}
\hline $\begin{array}{l}\text { SAMPLING } \\
\text { DATE }\end{array}$ & $\begin{array}{c}\text { DEPTH FROM } \\
\text { SURFACE }(\mathrm{cm} .)\end{array}$ & $\begin{array}{l}\text { DISTANCE } \\
\text { FROM } \\
\text { CENTER }\end{array}$ & $\begin{array}{l}\text { VOLUMETRIC } \\
\text { MOISTURE } \\
\text { CONTENT }(8)\end{array}$ \\
\hline $180 \mathrm{CT} 84$ & 295 & 40 & 27.1 \\
\hline $180 \mathrm{CT} 84$ & 295 & 50 & 27.0 \\
\hline $180 \mathrm{CT} 84$ & 295 & 60 & 26.7 \\
\hline $180 \mathrm{CT} 84$ & 295 & 70 & 26.9 \\
\hline $180 \mathrm{CT} 84$ & 295 & 80 & 26.4 \\
\hline $180 \mathrm{CT} 84$ & 295 & 90 & 26.0 \\
\hline $180 \mathrm{CT} 84$ & 295 & 100 & 26.9 \\
\hline $180 \mathrm{CT} 84$ & 295 & 110 & 26.5 \\
\hline $180 \mathrm{CT} 84$ & 295 & 120 & 25.5 \\
\hline $310 \mathrm{CT} 84$ & 295 & 0 & 28.4 \\
\hline $310 \mathrm{CT} 84$ & 295 & 10 & 26.8 \\
\hline $310 \mathrm{CT} 84$ & 295 & 20 & 27.1 \\
\hline 310 CT84 & 295 & 30 & 27.1 \\
\hline $310 \mathrm{CT} 84$ & 295 & 40 & 26.9 \\
\hline $310 \mathrm{CT} 84$ & 295 & 50 & 26.6 \\
\hline $310 \mathrm{CT} 84$ & 295 & 60 & 26.8 \\
\hline $310 \mathrm{CT} 84$ & 295 & 70 & 26.6 \\
\hline $310 \mathrm{CT} 84$ & 295 & 80 & 26.3 \\
\hline $310 \mathrm{CT} 84$ & 295 & 90 & 26.5 \\
\hline $310 \mathrm{CT} 84$ & 295 & 100 & 26.5 \\
\hline $310 \mathrm{CT} 84$ & 295 & 110 & 26.5 \\
\hline $310 \mathrm{CT} 84$ & 295 & 120 & 24.9 \\
\hline 28NOV84 & 295 & 0 & 28.8 \\
\hline 28 NOV84 & 295 & 10 & 27.4 \\
\hline 28NOV84 & 295 & 20 & 27.6 \\
\hline 28NOV84 & 295 & 30 & 27.7 \\
\hline 28NOV84 & 295 & 40 & 27.2 \\
\hline 28NOV84 & 295 & 50 & 27.2 \\
\hline 28NOV84 & 295 & 60 & 27.3 \\
\hline 28NOV84 & 295 & 70 & 27.6 \\
\hline 28NOV84 & 295 & 80 & 27.1 \\
\hline 28NOV84 & 295 & 90 & 26.9 \\
\hline 28NOV84 & 295 & 100 & 27.0 \\
\hline 28NOV84 & 295 & 110 & 27.3 \\
\hline 28 NOV 84 & 295 & 120 & 26.2 \\
\hline 03JAN85 & 295 & 0 & 28.5 \\
\hline 03JAN85 & 295 & 60 & 27.2 \\
\hline 03JAN85 & 295 & 120 & 26.0 \\
\hline 17JAN85 & 295 & 0 & 30.7 \\
\hline 17JAN85 & 295 & 60 & 28.7 \\
\hline 17JAN85 & 295 & 120 & 28.2 \\
\hline 04FEB 85 & 295 & 0 & 28.4 \\
\hline 04 FEB8 5 & 295 & 60 & 27.2 \\
\hline 04FEB85 & 295 & 120 & 25.3 \\
\hline $20 \mathrm{FEB} 85$ & 295 & 0 & 29.3 \\
\hline
\end{tabular}




\begin{tabular}{|c|c|c|c|}
\hline $\begin{array}{l}\text { SAMPLING } \\
\text { DATE }\end{array}$ & $\begin{array}{l}\text { DEPTH FROM } \\
\text { SURFACE }(\mathrm{cm} .)\end{array}$ & $\begin{array}{l}\text { DISTANCE } \\
\text { FROM } \\
\text { CENTER }\end{array}$ & $\begin{array}{l}\text { VOLUMETRIC } \\
\text { MOISTURE } \\
\text { CONTENT }\end{array}$ \\
\hline 20FEB8 5 & 295 & 60 & 27.8 \\
\hline 20FEB85 & 295 & 120 & 27.2 \\
\hline 06MAR85 & 295 & 0 & 30.0 \\
\hline 06MAR85 & 295 & 60 & 28.5 \\
\hline O6MAR 85 & 295 & 120 & 27.7 \\
\hline 21 MAR 85 & 295 & 0 & 30.7 \\
\hline 21MAR85 & 295 & 60 & 28.6 \\
\hline 21MAR8 5 & 295 & 120 & 27.7 \\
\hline OIAPR85 & 295 & 0 & 31.7 \\
\hline 01APR8 5 & 295 & 60 & 30.4 \\
\hline 01APR85 & 295 & 120 & 28.8 \\
\hline 08APR85 & 295 & 0 & 30.1 \\
\hline 08APR 85 & 295 & 60 & 28.0 \\
\hline 08APR85 & 295 & 120 & 27.1 \\
\hline 25APR85 & 295 & 0 & 28.8 \\
\hline 25APR85 & 295 & 60 & 26.8 \\
\hline 25APR85 & 295 & 120 & 25.7 \\
\hline 29APR85 & 295 & 0 & 29.3 \\
\hline 29APR85 & 295 & 60 & 27.0 \\
\hline 29APR85 & 295 & 120 & 26.3 \\
\hline 30APR85 & 295 & 0 & 29.0 \\
\hline 30APR85 & 295 & 60 & 27.0 \\
\hline 30APR85 & 295 & 120 & 25.9 \\
\hline 01MAY85 & 295 & 0 & 28.8 \\
\hline 01MAY85 & 295 & 60 & $27: 1$ \\
\hline O1MAY85 & 295 & 120 & 25.0 \\
\hline 02MAY85 & 295 & 0 & 28.8 \\
\hline 02MAY85 & 295 & 60 & 26.4 \\
\hline 02MAY85 & 295 & 120 & 23.1 \\
\hline 03MAY85 & 295 & 0 & 28.5 \\
\hline 03MAY85 & 295 & 60 & 26.6 \\
\hline 03MAY85 & 295 & 120 & 22.1 \\
\hline 06MAY85 & 295 & 0 & 27.8 \\
\hline 06MAY85 & 295 & 60 & 26.0 \\
\hline 06MAY85 & 295 & 120 & 23.2 \\
\hline 08MAY85 & 295 & 0 & 26.9 \\
\hline O8MAY85 & 295 & 60 & 25.4 \\
\hline 08MAY85 & 295 & 120 & 21.5 \\
\hline 10MAY85 & 295 & 0 & 26.8 \\
\hline 10MAY85 & 295 & 60 & 25.1 \\
\hline 10MAY85 & 295 & 120 & 21.0 \\
\hline 13MAY85 & 295 & 0 & 26.1 \\
\hline 13MAY85 & 295 & 60 & 24.6 \\
\hline 13MAY85 & 295 & 120 & 21.7 \\
\hline 15MAY85 & 295 & 0 & 25.6 \\
\hline
\end{tabular}




\section{SAMPLING \\ DATE}

15MAY85

15MAY85

17 MAY85

17 MAY85

17MAY85

21MAY85

21MAY85

21MAY85

23MAY85

23 MAY85

23 MAY85

28 MAY 85

28MAY85

28MAY85

30MAY 85

30MAY85

30MAY85

04JUN85

04JUN85

04JUN85

12 JUN85

12JUN85

12JUN85

$18 J U N 85$

18JUN85

18JUN85

25JUN85

25JUN85

25JUN85

02JUL85

02JUL85

02JUL85

11JUL85

11JUL85

11JUL85

12JUL85

12JUL85

12JUL85

13JUL85

13JUL85

13JUL85

14JUL85

14JUL85

14JUL8 5

15JUL85

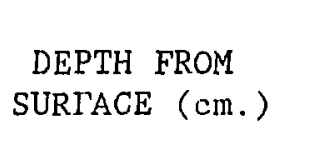

295

295

295

295

295

295

295

295

295

295

295

295

295

295

295

295

295

295

295

295

295

295

295

295

295

295

295

295

295

295

295

295

295

295

295

295

295

295

295

295

295

295

295

295

295

$\begin{array}{ll}\text { DISTANCE } & \text { VOLUMETRIC } \\ \text { FROM } & \text { MOISTURE } \\ \text { CENTER } & \text { CONTENT (q) }\end{array}$

60
120
0

23.9

21.3

25.4

23.8

21.0

24.1

22.4

19.6

23.8

22.4

19.2

22.4

21.2

18.3

21.7

20.9

18.4

22.1

20.7

18.1

21.2

20.1

17.3

21.0

20.2

17.2

21.1

19.9

16.7

20.6

20.1

16.1

20.2

19.5

16.4

20.0

18.7

16.3

19.5

19.0

15.6

19.7

18.7

15.2

19.6 


\section{SAMPLING DATE}

15JUL85

15JUL85

16JUL85

16JUL85

16JUL85

17 JUL85

17JUL85

17JUL85

18JUL85

18 JUL85

18JUL85

19JUL85

19JUL85

19JUL85

21 JUL85

21JUL85

21JUL85

22JUL85

22JUL85

22JUL85

23JUL85

23JUL85

23JUL85

24JUL85

24JUL85

24JUL85

25JUL85

25JUL85

25JUL85

26JUL85

26JUL85

26JUL85

27JUL85

27JUL85

27JUL85

28JUL85

28JUL85

28JUL85

29JUL85

29JUL85

29JUL8 5

30 JUL8 5

30 JUL85

30 JUL8 5

02 AUG85

\author{
DEPTH FROM \\ SURFACE $(\mathrm{cm}$.
}

295

295

295

295

295

295

295

295

295

295

295

295

295

295

295

295

295

295

295

295

295

295

295

295

295

295

295

295

295

295

295

295

295

295

295

295

295

295

295

295

295

295

295

295

295

$\begin{array}{ll}\text { DISTANCE } & \text { VOLUMETRIC } \\ \text { FROM } & \text { MOISTURE } \\ \text { CENTER } & \text { CONTENT }(q)\end{array}$

18.9

12.6

19.2

18.7

11.4

20.2

18.9

15.5

21.6

20.1

16.5

25.8

24.3

19.8

27.4

25.9

24.2

27.1

26.0

24.2

27.6

25.8

24.0

26.9

26.0

23.8

27.7

25.7

22.6

27.6

26.0

24.2

28.0

26.1

24.8

27.8

25.8

24.7

27.8

26.3

24.5

28.0

26.2

24.4

26.4 


\begin{tabular}{|c|c|c|c|}
\hline $\begin{array}{l}\text { SAMPLING } \\
\text { DATE }\end{array}$ & $\begin{array}{l}\text { DEPTH FROM } \\
\text { SURFACE (cm.) }\end{array}$ & $\begin{array}{l}\text { DISTANCE } \\
\text { FROM } \\
\text { CENTER }\end{array}$ & $\begin{array}{l}\text { VOLUMETR } \\
\text { MOISTURE } \\
\text { CONTENT }\end{array}$ \\
\hline 02AUG8 5 & 295 & 60 & 26.1 \\
\hline 02AUG8 5 & 295 & 120 & 24.6 \\
\hline 04AUG85 & 295 & 0 & 27.6 \\
\hline 04AUG85 & 295 & 60 & 25.9 \\
\hline 04AUG85 & 295 & 120 & 24.9 \\
\hline 06AUG85 & 295 & 0 & 28.2 \\
\hline 06AUG85 & 295 & 60 & 26.6 \\
\hline 06AUG85 & 295 & 120 & 25.1 \\
\hline 09AUG85 & 295 & 0 & 27.9 \\
\hline 09AUG85 & 295 & 60 & 26.3 \\
\hline 09AUG85 & 295 & 120 & 25.1 \\
\hline 14AUG85 & 295 & 0 & 28.3 \\
\hline 14AUG85 & 295 & 60 & 26.3 \\
\hline 14AUG85 & 295 & 120 & 24.6 \\
\hline 16AUG85 & 295 & 0 & 28.2 \\
\hline 16AUG85 & 295 & 60 & 26.7 \\
\hline 16AUG85 & 295 & 120 & 24.7 \\
\hline 21AUG85 & 295 & 0 & 28.6 \\
\hline 21AUG85 & 295 & 60 & 26.9 \\
\hline 21AUG85 & 295 & 120 & 25.2 \\
\hline 23AUG85 & 295 & 0 & 28.2 \\
\hline 23AUG85 & 295 & 60 & 26.6 \\
\hline 23AUG85 & 295 & 120 & 24.9 \\
\hline 27AUG85 & 2.95 & 0 & 28.8 \\
\hline 27AUG85 & 295 & 60 & 26.7 \\
\hline 27AUG85 & 295 & 120 & 24.9 \\
\hline 30AUG85 & 295 & 0 & 27.9 \\
\hline 30AUG85 & 295 & 60 & 26.7 \\
\hline 30AUG85 & 295 & 120 & 24.9 \\
\hline 03SEP85 & 295 & 0 & 28.4 \\
\hline 03SEP85 & 295 & 60 & 27.3 \\
\hline 03SEP85 & 295 & 120 & 25.4 \\
\hline 09SEP85 & 295 & 0 & 28.5 \\
\hline 09SEP85 & 295 & 60 & 26.9 \\
\hline 09SEP85 & 295 & 120 & 25.1 \\
\hline 18SEP85 & 295 & 0 & 28.5 \\
\hline 18SEP85 & 295 & 60 & 26.8 \\
\hline 18SEP85 & 295 & 120 & 25.3 \\
\hline 30SEP85 & 295 & 0 & 28.6 \\
\hline 30 SEP85 & 295 & 60 & 26.4 \\
\hline 30 SEP85 & 295 & 120 & 25.1 \\
\hline 090СT85 & 295 & 0 & 28.4 \\
\hline 090СT85 & 295 & 60 & 26.9 \\
\hline 090СT85 & 295 & 120 & 25.3 \\
\hline 160CT85 & 295 & 0 & 29.0 \\
\hline
\end{tabular}


SAMPLING
DATE

$160 \mathrm{CT} 85$

$160 \mathrm{CT} 85$

$230 \mathrm{CT} 85$

$230 \mathrm{CT} 85$

$230 \mathrm{CT} 85$

$300 \mathrm{CT} 85$

$300 \mathrm{CT} 85$

$300 \mathrm{CT} 85$

06NOV85

06NOV85

06NOV85

DEPTH FR
SURFACE
295
295
295
295
295
295
295
295
295
295
295

DEPTH FROM

295

295

295

295

295

295

295

295

295

295
DISTANCE VOLUMETRIC

FROM

CENTER

60

120

0

60

120

0

60

120

0

60

120
MOISTURE

CONTENT (q)

26.2

25.2

28.7

27.0

25.6

28.4

26.8

25.1

28.4

26.6

25.3 


\begin{tabular}{|c|c|c|c|}
\hline SAMPLING & DEPTH FROM & $\begin{array}{l}\text { DISTANCE } \\
\text { EROM }\end{array}$ & $\begin{array}{l}\text { VOLUMETRIC } \\
\text { MOISTURE }\end{array}$ \\
\hline DATE & SURFACE $(\mathrm{cm})$. & CENTER & CONTENT (z) \\
\hline 12APR84 & 370 & 0 & 22.4 \\
\hline 12APR84 & 370 & 10 & 21.0 \\
\hline 12APR84 & 370 & 20 & 21.2 \\
\hline 12APR84 & 370 & 30 & 21.0 \\
\hline 12APR84 & 370 & 40 & 21.7 \\
\hline 12APR8 4 & 370 & 50 & 21.8 \\
\hline 12APR84 & 370 & 60 & 21.7 \\
\hline 12APR84 & 370 & 70 & 22.1 \\
\hline 12APR 84 & 370 & $8 n$ & 21.3 \\
\hline 12APR84 & 370 & 90 & 20.9 \\
\hline 12APR84 & 370 & 100 & 20.7 \\
\hline 12APR84 & 370 & 110 & 20.0 \\
\hline 12APR84 & 370 & 120 & 18.6 \\
\hline 12APR84 & 370 & 130 & 12.8 \\
\hline 10MAY84 & 370 & 0 & 20.9 \\
\hline 10MAY84 & 370 & 10 & 20.3 \\
\hline 10MAY84 & 370 & 20 & 20.4 \\
\hline 10MAY84 & 370 & 30 & 20.2 \\
\hline IOMAY84 & 370 & 40 & 20.5 \\
\hline 10MAY84 & 370 & 50 & 20.5 \\
\hline 10MAY84 & 370 & 60 & 20.8 \\
\hline 10MAY84 & 370 & 70 & 20.4 \\
\hline 10MAY84 & 370 & 80 & 20.6 \\
\hline 10MAY84 & 370 & 90 & 19.8 \\
\hline 10MAY84 & 370 & 100 & 19.2 \\
\hline 10MAY84 & 370 & 110 & 19.2 \\
\hline 10MAY84 & 370 & 120 & 18.0 \\
\hline 06JUN84 & 370 & 0 & 26.2 \\
\hline 06JUN84 & 370 & 10 & 25.9 \\
\hline 06JUN84 & 370 & 20 & 26.0 \\
\hline 06JUN84 & 370 & 30 & 26.2 \\
\hline 06JUN84 & 370 & 40 & 26.4 \\
\hline 06JUN84 & 370 & 50 & 26.5 \\
\hline 06JUN84 & 370 & 60 & 26.0 \\
\hline 06JUN84 & 370 & 70 & 26.6 \\
\hline 06JUก 84 & 370 & 80 & 26.2 \\
\hline 06JUN84 & 370 & 90 & 27.2 \\
\hline 06JUnY84 & 370 & 100 & 27.9 \\
\hline 06JUN84 & 370 & 110 & 28.0 \\
\hline 06JUN84 & 370 & 120 & 26.8 \\
\hline 25JUL84 & 370 & 0 & 31.5 \\
\hline $25 \mathrm{JUL} 84$ & 370 & 10 & 29.6 \\
\hline 25JUL84 & 370 & 20 & 29.2 \\
\hline 25JUL84 & 370 & 30 & 28.5 \\
\hline 25JUL84 & 370 & 40 & 27.9 \\
\hline
\end{tabular}




\section{SAMPLING DATE}

25JUL84

25JUL84

25JUL84

25JUL84

$25 J U L 84$

2.5JUL84

25JUL84

25JUL84

09AUG84

09AUG84

09AUG84

09AUG84

09AUG84

O9AUG84

09AUG84

09AUG84

09 AUG84

09AUG 84

09AUG84

09AUG84

09AUG84

09AUG84

09AUG84

09AUG84

09AUG84

$09 A U G 84$

09AUG84

09AUG84

09AUG84

$09 A U G 84$

09AUG84

09AUG84

09AUG84

09AUG84

20AUG 84

20AUG84

20AUG84

20AUG 84

20AUG84

20AUG84

20AUG84

20AUG84

20AUG84

20AUG84

20AUG84
DEPTH FROM

SURFACE (cm.)

370

370

370

370

370

370

370

370

370

370

370

370

370

370

370

370

370

370

370

370

370

370

370

370

370

370

370

370

370

370

370

370

370

370

370

370

370

370

370

370

370

370

370

370

370

\section{DISTANCE}

FROM

CENTER

50

60

70

80

90

100

110

120

0

0

10

10

$2 C$

20

30

30

40

40

50

50

60

60

70

70

80

80

90

90

100

100

110

110

120

120

0

10

20

30

40

50

60

70

80

90

100
VOLUMETRIC

MOISTURE

CONTENT (\%)

27.6

27.0

27.3

27.9

27.8

28.4

28.9

28.1

34.0

32.7

31.1

32.3

31.8

30.6

31.4

30.3

31.1

30.0

30.4

29.3

30.1

29.1

29.2

30.2

29.2

30.3

30.6

29.5

30.9

29.8

30.6

31.8

30.1

31.2

35.4

33.9

33.0

32.3

31.5

31.6

31.0

30.6

31.1

31.5

31.8 


\section{SAMPLING \\ DATE}

20AUG84

20AUG84

04SEP84

04SEP 84

04SEP84

04SEP84

04SEP84

04SEP84

04SEP84

04SEP84

04SEP84

04SEP84

04SEP84

04SEP84

D4SEP 84

18 SEP84

18 SEP 84

$18 \mathrm{SEP} 84$

$18 \mathrm{SEP} 84$

$18 \mathrm{SEP} 84$

$18 \mathrm{SEP} 84$

18 SEP 84

18SEP 84

18SEP 84

18SEP 84

18SEP 84

18SEP 84

$18 S E P 84$

$020 \mathrm{CT} 84$

$020 \mathrm{CT} 84$

$020 \mathrm{CT} 84$

$020 \mathrm{CT} 84$

$020 \mathrm{CT} 84$

020CT84

$020 \mathrm{CT} 84$

$020 \mathrm{CT} 84$

020CT 84

020CT 84

$020 \mathrm{CT} 84$

020CT84

$020 \mathrm{CT} 84$

180CT 84

$180 \mathrm{CT} 84$

$180 \mathrm{CT} 84$

180CT 84

$\begin{array}{cl}\text { DEPTH FROM } & \text { DISTANCE } \\ \text { SURFACE (cm. }) & \text { FROM } \\ \text { CENTER }\end{array}$

370

370

370

370

370

370

370

370

370

370

370

370

370

370

370

370

370

370

370

370

370

370

370

370

370

370

370

370

370

370

370

370

370

370

370

370

370

370

370

370

370

370

370

370

370
110

120

0

10

20

30

40

50

60

70

80

90

100

110

120

0

10

20

30

40

50

60

70

80

90

100

110

120

0

10

20

30

40

50 .

60

70

80

90

100

110

120

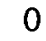

10

20

30
VOLUMETRIC

MOISTURE

CONTENT ( 8 )

32.8

31.8

34.4

32.5

32.1

31.6

30.7

30.4

30.0

29.6

30.4

30.4

31.0

31.8

31. 3

31.2

30.1

29.3

29.0

28.5

28.1

27.8

27.8

28.4

28.4

29.1

29.0

28.5

29.5

28.7

28.7

28.2

27.8

27.5

27.4

27.1

27.8

28.2

28.5

28.6

26.9

31.2

29.6

29.7

28.9 
SAMPLING

DATE

$180 \mathrm{CT} 84$

$180 \mathrm{CT} 84$

$180 \mathrm{CT} 84$

$180 \mathrm{CT} 84$

$180 \mathrm{CT} 84$

$180 \mathrm{CT} 84$

$180 \mathrm{CT} 84$

$180 \mathrm{CT} 84$

$180 \mathrm{CT} 84$

$310 \mathrm{CT} 84$

$310 \mathrm{CT} 84$

$310 \mathrm{CT} 84$

$310 \mathrm{CT} 84$

$310 \mathrm{CT} 84$

$310 \mathrm{CT} 84$

310CT 84

310 CT 84

310 CT 84

$310 \mathrm{CT} 84$

310CT 84

310CT84

$310 \mathrm{CT} 84$

28NOV84

28 NOV 84

28 NOV 84

28NOV 84

28NOV84

28NOV84

28NOV84

28NOV84

28NOV 84

28NOV84

28NOV84

28NOV84

28NOV84

03JAN85

03JAN85

03JAN85

17JAN85

17JAN85

17JAN85

04FEB85

04FEB85

04FEB 85

20FEB85

$\begin{array}{cl} & \text { DISTANCE } \\ \text { DEPTH FROM } & \text { FROM } \\ \text { SURFACE (cm.) } & \text { CENTER }\end{array}$

370

370

370

370

370

370

370

370

370

370

370

370

370

370

370

370

370

370

370

370

370

370

370

370

370

370

370

370

370

370

370

370

370

370

370

370

370

370

370

370

370

370

370

370

370
40

50

60

70

80

90

100

110

120

0

10

20

30

40

50

60

70

80

90

100

110

120

0

10

20

30

40

50

60

70

80

90

100

110

120

0

60

120

0

60

120

0

60

120

0
VOLUMETRIC

MOISTURE

CONTENT

( 8 )
28.4

28.0

27.7

28.1

28.1

28.9

29.4

29.4

27.7

30.7

30.1

29.7

29.0

28.8

28.2

27.6

27.8

28.0

28.4

29.0

29.5

28.8

31.4

30.2

29.8

29.4

28.9

28.3

28.1

28.0

28.1

28.6

29.5

29.5

28.9

29.1

27.1

26.6

33.0

28.7

31.0

31.5

28.4

28.8

31.9 
SAMPLING

DATE

20FEB 85

20FEB 85

06MAR 85

O6MAR85

06MAR85

21MAR 85

21MAR85

21MAR85

01APR85

O1APR 85

01APR 85

08APR 85

08APR85

08APR 85

25APR 85

25APR 85

25APR 85

29APR85

29APR85

29APR 85

30APR 85

30APR 85

30APR 85

01MAY85

01MAY85

01MAY85

02MAY85

02 MAY8 5

02 MAY85

03 MAY85

03MAY85

03MAY85

06MAY85

06MAY85

06MAY85

08 MAY85

08 MAY85

08MAY85

10MAY 85

10MAY85

10MAY85

13MAY85

13MAY85

13MAY85

15MAY85

DEPTH FROM
SURFACE (cm.)

370

370

370

370

370

370

370

370

370

370

370

370

370

370

370

370

370

370

370

370

370

370

370

370

370

370

370

370

370

370

370

370

370

370

370

370

370

370

370

370

370

370

370

370

370 $\begin{array}{ll}\text { DISTANCE } & \text { VOLUMETRIC } \\ \text { FROM } & \text { MOISTURE } \\ \text { CENTER } & \text { CONTENT }(\xi)\end{array}$

60
120

0

60

120

0

60

120

0

60

120

0

60

120

0

60

120

0

60

120

0

60

120

0

60

120

0

60

120

0

60

120

0

60

120

0

60

120

0

60

120

0

60

120

0
28.2

30.0

32.1

29.1

29.8

32.9

29.1

29.0

34.5

30.3

31.7

32.3

29.0

30.0

31.2

27.6

28.2

31.2

28.0

28.5

31.8

27.9

29.1

31.3

28.3

$27 . ?$

31.2

28.2

26.3

31.2

28.0

25.5

30.0

27.7

27.3

28.4

27.6

23.2

27.2

25.8

20.8

25.6

24.0

21.9

24.6 


\section{SAMPLING \\ DATE}

15MAY85
15 MAY85
17 MAY85
17 MAY 85
17 MAY85
21 MAY85
21 MAY85
21MAY85
23 MAY85
23MAY85

23 MAY 85

$28 M A Y 85$

28MAY85

28MAY85

30MAY85

30MAY85

30MAY85

04JUN8 5

04JUN85

04JUN85

12JUN85

12JUN85

12 JUN85

18JUN85

$18 J$ UN8 5

$18 J$ UN 85

25JUN8 5

25JUN85

25JUN85

02JUL85

02JUL.85

02JULB5

11JUL85

11JUL85

11JUL85

12JUL85

12 JUL85

12JUL85

13JUL85

13JUL85

13JUL85

14JUL85

14JUL85

14JUL85

15JUL85

\section{DEPTH FROM \\ SURFACE (cm.)}

370

370

370

370

370

370

370

370

370

370

370

370

370

370

370

370

370

370

370

370

370

370

370

370

370

370

370

370

370

370

370

370

370

370

370

370

370

370

370

370

370

370

370

370

370

$\begin{array}{ll}\text { DISTANCE } & \text { VOLUMETRIC } \\ \text { FROM } & \text { MOISTURE } \\ \text { CENTER } & \text { CONTENT (g) }\end{array}$

60

120

23.4

20.9

24.0

23.3

21.5

23.4

22.5

19.8

22.6

22.2

19.9

22.1

21.6

19.5

22.2

21.7

18.5

21.9

21.5

18.3

21.8

21.2

18.5

21.3

21.0

17.9

21.3

21.2

18. 2

21.3

20.8

18.4

21.0

20.6

17.7

20.6

20.0

17.0

20.0

20.0

17.4

20.2

20.2

16.8

19.7 


\section{SAMPLING DATE}

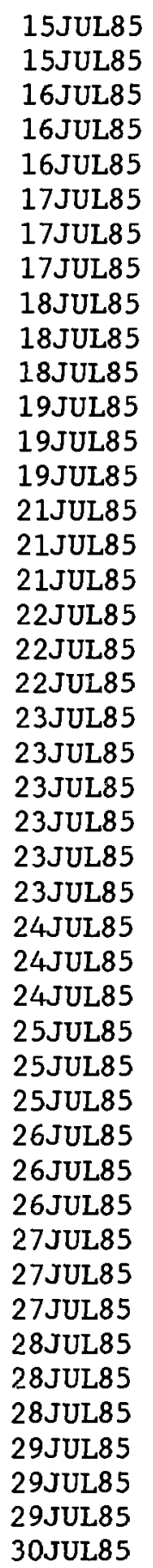

$\begin{array}{cl} & \text { DISTANCE } \\ \text { DEPTH FROM } & \text { FROM } \\ \text { SURFACE (cm.) } & \text { CENTER }\end{array}$

370

370

370

370

370

370

370

370

370

370

370

370

370

370

370

370

370

370

370

370

370

370

370

370

370

370

370

370

370

370

370

370

370

370

370

370

370

370

370

370

370

370

370

370

370
12

60

120

0

60

120

0

60

120

0

60

120

0

60

120

0

60

120

0

0

60

60

120

120

0

60

120

0

60

120

0

60

120

0

60

120

0

60

120

0

60

120

0
VOLUMETRIC

MOISTURE

CONTENT (8)

20.2

12.0

20.1

20.0

9.9

20.3

20.4

16.7

20.5

20.3

16.7

20.8

20.3

9.9

27.2

25.1

25.6

29.6

25.7

26.6

29.0

29.0

26.1

26.1

26.1

26.1

29.6

26.4

26.7

29.9

26.3

26.5

29.7

26.0

27.5

29.7

26.4

27.2

29.8

26.4

27.8

30.4

26.3

26.9

30.4 


\begin{tabular}{|c|c|c|c|}
\hline $\begin{array}{l}\text { SAMPLING } \\
\text { DATE }\end{array}$ & $\begin{array}{c}\text { DEPTH FROM } \\
\text { SURFACE }(\mathrm{cm} .)\end{array}$ & $\begin{array}{l}\text { DISTANCE } \\
\text { FROM } \\
\text { CENTER }\end{array}$ & $\begin{array}{l}\text { VOLUMETRIC } \\
\text { MOISTURE } \\
\text { CONTENT }\end{array}$ \\
\hline 30JUL85 & 370 & 60 & 26.0 \\
\hline 30JUL85 & 370 & 120 & 26.6 \\
\hline 02AUG85 & 370 & 0 & 29.6 \\
\hline 02AUG85 & 370 & 60 & 26.4 \\
\hline 02AUG 85 & 370 & 120 & 26.9 \\
\hline 04AUG85 & 370 & 0 & 29.7 \\
\hline 04 AUG85 & 370 & 60 & 26.6 \\
\hline 04AUG85 & 370 & 120 & 27.2 \\
\hline 06AUG85 & 370 & 0 & 30.4 \\
\hline 06AUG85 & 370 & 60 & 26.6 \\
\hline 06AUG85 & 370 & 120 & 27.2 \\
\hline 09AUG85 & 370 & 0 & 30.9 \\
\hline 09AUG85 & 370 & 60 & 26.5 \\
\hline 09AUG85 & 370 & 120 & 28.2 \\
\hline 14AUG85 & 370 & 0 & 30.7 \\
\hline 14AUG85 & 370 & 60 & 26.6 \\
\hline 14AUG85 & 370 & 120 & 27.7 \\
\hline 16AUG85 & 370 & 0 & 30.4 \\
\hline 16AUG85 & 370 & 60 & 26.5 \\
\hline 16AUG85 & 370 & 120 & 27.7 \\
\hline 21AUG85 & 370 & 0 & 31.0 \\
\hline 21AUG85 & 370 & 60 & 26.6 \\
\hline 21AUG85 & 370 & 120 & 27.4 \\
\hline 23AUG85 & 370 & 0 & 30.6 \\
\hline 23AUG85 & 370 & 60 & 26.4 \\
\hline 23AUG85 & 370 & 120 & 27.7 \\
\hline 27AUG85 & 370 & 0 & 30.7 \\
\hline 27AUG85 & 370 & 60 & 27.3 \\
\hline 27AUG85 & 370 & 120 & 27.6 \\
\hline 30AUG85 & 370 & 0 & 30.8 \\
\hline 30AUG85 & 370 & 60 & 27.3 \\
\hline 30AUG85 & 370 & 120 & 27.8 \\
\hline 03SEP85 & 370 & 0 & 30.7 \\
\hline 03SEP85 & 370 & 60 & 26.6 \\
\hline 03SEP85 & 370 & 120 & 28.0 \\
\hline 09SEP85 & 370 & 0 & 30.5 \\
\hline 09SEP85 & 370 & 60 & 27.0 \\
\hline 09SEP85 & 370 & 120 & 28.1 \\
\hline 18SEP85 & 370 & 0 & 30.8 \\
\hline 18SEP85 & 370 & 60 & 26.8 \\
\hline 13SEP85 & 370 & 120 & 28.2 \\
\hline 30SEP85 & 370 & 0 & 31.2 \\
\hline 30SEP85 & 370 & 60 & 27.0 \\
\hline 30SEP85 & 370 & 120 & 27.7 \\
\hline 090CT85 & 370 & 0 & 31.0 \\
\hline
\end{tabular}




\section{SAMPLING \\ DATE}

$090 \mathrm{CT} 85$

$090 \mathrm{CT} 85$

$160 \mathrm{CT} 85$

$160 \mathrm{CT} 85$

$160 \mathrm{CT} 85$

$230 \mathrm{CT} 85$

$230 \mathrm{CT} 85$

$230 \mathrm{CT} 85$

$300 \mathrm{CT} 85$

$300 \mathrm{CT} 85$

$300 \mathrm{CT} 85$

06 NOV85

06 NOV85

06 NOV 85

\section{DEPTH FROM \\ SURFACE (cm.) \\ DISTANCE \\ FROM \\ CENTER}

370

370

370

370

370

370

370

370

370

370

370

370

370

370
60

120

0

60

120

0

60

120

0

60

120

0

60

120
VOLUMETRIC

MOISTURE

CONTENT $(z)$

26.9

27.8

31.7

27. 1

27.9

31.0

26.9

28.3

31.0

27.4

28.0

31.5

27.4

27.6 


\section{SAMPLING DATE}

12APR 84

12APR 84

12APR84

12APR84

12APR 84

12APR84

12APR84

12APR84

12APR8 4

12APR84

12APR 84

12APR 84

12APR84

12APR 84

10MAY84

1OMAY 84

10MAY84

10MAY84

10MAY84

10MAY84

10MAY84

10MAY84

10MAY84

10MAY84

10MAY84

10MAY84

10MAY84

06JUN84

06JUN84

06JUN84

06JUN84

06JUN84

06JUN84

06JUN84

06JUN84

06JUN 84

06JUN84

06JUN84

06JUN84

06JUN84

25JUL84

25JUL84

25 JUL84

25JUL84

25JUL84

\section{DEPTH FROM}

SURFACE $(\mathrm{cm}$.

445

445

445

445

445

445

445

445

445

445

445

445

445

445

445

445

445

445

445

445

445

445

445

445

445

445

445

445

445

445

445

445

445

445

445

445

445

445

445

445

445

445

445

445

445

$\begin{array}{ll}\text { DISTANCE } & \text { VOLUMETRIC } \\ \text { FROM } & \text { MOISTURE } \\ \text { CENTER } & \text { CONTENT }(\xi)\end{array}$

25.2

25. 1

24.7

25.0

24.3

24.1

23.8

23.2

23.3

23.6

22.8

22.3

20.7

15.2

23.9

23.3

23.2

23.3

23.2

22.9

22.9

22.5

21.9

22.2

21.9

21.0

19.3

25.4

24.7

25.1

24.7

25.2

24.6

23.8

23.6

23.9

23.8

23.4

22.7

20.7

29.6

27.6

27.9

27.3

27.4 


\section{SAMPLING \\ DATE}

25JUL84

25JUL84

25JUL84

25JUL84

25JUL84

25JUL84

25JUL84

25JUL84

09AUG84

$09 A U G 84$

09AUG84

09AUG84

09AUG84

09AUG84

09AUG84

09AUG84

09AUG84

09AUG84

09AUG84

09AUG84

09AUG84

09AUG84

09AUG84

09AUG84

OYAUG84

09AUG84

09AUG84

09AUG84

09AUG84

09AUG84

09AUG84

09AUG84

09AUG84

09AUG84

20AUG84

20AUG84

20AUG84

20AUG84

20AUG84

20AUG84

20AUG84

20AUG84

20AUG84

20AUG84

20AUG84

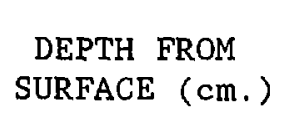

445

445

445

445

445

445

445

445

445

445

445

445

445

445

445

445

445

445

445

445

445

445

445

445

445

445

445

445

445

445

445

445

445

445

445

445

445

445

445

445

445

445

445

445

445

$\begin{array}{ll}\text { DISTANCE } & \text { VOLUMETRIC } \\ \text { FROM } & \text { MOISTURE } \\ \text { CENTER } & \text { CONTENT }(\xi)\end{array}$

27.3

27.5

27.6

27.3

27.5

28.1

28.2

27.2

32.2

31.1

31.0

29.9

30.9

29.8

30.5

29.4

29.4

30.5

29.2

30.3

30.1

29.1

31.0

29.9

29.2

30.2

29.4

30.5

30.1

31.2

32.0

30.9

29.4

30.5

33.2

31.5

30.6

31.1

30.6

30.7

31.3

30.7

30.7

30.6

31.1 


\begin{tabular}{|c|c|c|c|}
\hline $\begin{array}{l}\text { SAMPLING } \\
\text { DATE }\end{array}$ & $\begin{array}{l}\text { DEPTH FROM } \\
\text { SURFACE }(\mathrm{cm} .)\end{array}$ & $\begin{array}{l}\text { DISTANCE } \\
\text { FROM } \\
\text { CENTER }\end{array}$ & $\begin{array}{l}\text { VOLUMETRIC } \\
\text { MOISTURE } \\
\text { CONTENT }\end{array}$ \\
\hline 20AUG84 & 445 & 110 & 31.4 \\
\hline 20AUG84 & 445 & 120 & 30.0 \\
\hline 04SEP 84 & 445 & 0 & 31.8 \\
\hline 04SEP84 & 445 & 10 & 29.9 \\
\hline 04SEP84 & 445 & 20 & 29.9 \\
\hline 04SEP84 & 445 & 30 & 29.6 \\
\hline 04SEP84 & 445 & 40 & 30.3 \\
\hline 04SEP84 & 445 & 50 & 29.7 \\
\hline 04SEP84 & 445 & 60 & 30.0 \\
\hline 04SEP84 & 445 & 70 & 29.7 \\
\hline 04SEP84 & 445 & 80 & 29.6 \\
\hline 04SEP84 & 445 & 90 & 29.5 \\
\hline 04SEP84 & 445 & 100 & 29.7 \\
\hline 04SEP84 & 445 & 110 & 30.0 \\
\hline 04 SEP84 & 445 & 120 & $28.7 !$ \\
\hline 18SEP84 & 445 & 0 & 29.6 \\
\hline 18SEP84 & 445 & 10 & 27.8 \\
\hline 18SEP84 & 445 & 20 & 27.3 \\
\hline 18SEP84 & 445 & 30 & 27.9 \\
\hline 18SEP84 & 445 & 40 & 27.3 \\
\hline 18SEP84 & 445 & 50 & 27.5 \\
\hline 18SEP84 & 445 & 60 & 27.4 \\
\hline 18SEP84 & 445 & 70 & 26.9 \\
\hline 18SEP84 & 445 & 80 & 22.3 \\
\hline 18SEP84 & 445 & 90 & 27.2 \\
\hline 18SEP84 & 445 & 100 & 27.8 \\
\hline 18SEP 84 & 445 & 110 & 27.5 \\
\hline 18SEP84 & 445 & 120 & 26.3 \\
\hline 020CT 84 & 445 & 0 & 28.3 \\
\hline $020 \mathrm{CT} 84$ & 445 & 10 & 26.8 \\
\hline 020CT84 & 445 & 20 & 26.8 \\
\hline 020CT84 & 445 & 30 & 26.6 \\
\hline 020CT84 & 445 & 40 & 26.4 \\
\hline 020CT 84 & 445 & 50 & 26.4 \\
\hline 020CT84 & 445 & 60 & 26.3 \\
\hline 020CT84 & 445 & 70 & 26.0 \\
\hline $020 \mathrm{CT} 84$ & 445 & 80 & 25.8 \\
\hline 020CT84 & 445 & 90 & 25.7 \\
\hline C20CT 84 & 445 & 100 & 25.6 \\
\hline 020CT84 & 445 & 110 & 24.9 \\
\hline 020СТ84 & 445 & 120 & 22.8 \\
\hline $180 \mathrm{CT} 94$ & 445 & 0 & 29.1 \\
\hline $180 \mathrm{CT} 84$ & 445 & 10 & 27.9 \\
\hline $180 \mathrm{CT} 84$ & 445 & 20 & 26.9 \\
\hline $180 \mathrm{CT} 84$ & 445 & 30 & 27.4 \\
\hline
\end{tabular}


SAMPLING
DATE

$180 \mathrm{CT} 84$

$180 \mathrm{CT} 84$

$180 \mathrm{CT} 84$

$180 \mathrm{CT} 84$

$180 \mathrm{CT} 84$

$180 \mathrm{CT} 84$

$180 \mathrm{CT} 84$

$180 \mathrm{CT} 84$

$180 \mathrm{CT} 84$

$310 \mathrm{CT} 84$

$310 \mathrm{CT} 84$

310 CT 84

$310 \mathrm{CT} 84$

$310 \mathrm{CT} 84$

310CT84

$310 \mathrm{CT} 84$

$310 \mathrm{CT} 84$

$310 \mathrm{CT} 84$

310CT 84

$310 \mathrm{CT} 84$

310 TT 84

310 CT 84

28NOV84

28NOV 84

28NOV84

28NOV84

28NOV84

28 NOV84

28NOV84

28NOV84

28NOV 84

28NOV84

28NOV84

28NOV84

28NOV84

17JAN85

17JAN85

17JAN85

04FEB85

04FEB 85

04FEB8 5

20FEB 85

20FEB85

20FEB 85

06MAR8 5

$\begin{array}{cl} & \text { DISTANCE } \\ \text { DEPTH FROM } & \text { FROM } \\ \text { SURFACE (cm.) } & \text { CENTER }\end{array}$

445

445

445

445

445

445

445

445

445

445

445

445

445

445

445

445

445

445

445

445

445

445

445

445

445

445

445

445

445

445

445

445

445

445

445

445

445

445

445

445

445

445

445

445

445
VOLUMETRIC

MOISTURE

CONTENT

(8)
27.5

27.1

27.6

26.8

27.1

27.1

27.3

26.8

25.4

29.4

27.9

28.0

27.6

28.0

26.9

27.3

27.1

27.5

27.2

27.2

26.6

26.0

29.3

28.3

27.7

28.1

27.7

27.5

27.7

27.6

27.3

27.0

27.7

27.8

26.8

31.3

28.6

28.9

29.1

27.1

26.1

30.5

28.5

27,8

30,4 
SAMPLING

DATE

06MAR85

06MAR85

21MAR85

21MAR85

21MAR85

OIAPR 85

OIAPR 85

OIAPR 85

O8APR8 5

08APR85

O8APR 85

25APR85

25APR 85

25APR85

30APR 85

30 APR 85

$30 A P R 85$

O2MAY85

$02 M A Y 85$

02 MAY 85

03 MAY 85

03 MAY85

03MAY85

06MAY85

06 MAY85

06MAY85

08MAY85

08MAY85

O8MAY85

10MAY85

10MAY 85

10MAY85

13 MAY 85

13 MAY85

13 MAY85

15MAY85

15MAY85

15MAY85

17MAY85

17MAY85

17MAY85

21MAY85

21MAY85

21MAY85

23MAY85
DEPTH FROM

SURFACE (cm.)

445

445

445

445

445

445

445

445

445

445

445

445

445

445

445

445

445

445

445

445

445

445

445

445

445

445

445

445

445

445

445

445

445

445

445

445

445

445

445

445

445

445

445

445

445

$\begin{array}{ll}\text { DISTANCE } & \text { VOLUMETRIC } \\ \text { FROM } & \text { MOISTURE } \\ \text { CENTER } & \text { CONTENT (\&) }\end{array}$

28.9

27.8

31.0

29.1

29.3

32.7

30.3

30.1

30.6

28.3

27.9

28.6

27.3

26.5

30.2

27.8

27.1

29.2

27.5

24.3

29.5

27.3

23.6

28.5

26.8

23.5

27.7

26.0

20.4

27.4

25.8

20.8

26.8

25.3

21.4

26.6

24.7

21.5

26.4

24.4

21.5

25.7

23.9

20.7

25.4 


\section{SAMPLING \\ DATE}

23MAY85
23 MAY85
28MAY85
28MAY85
28MAY85

30MAY85

30 MAY 85

30MAY85

04JUN85

$04 \mathrm{JUN} 85$

04JUN85

12 JUN85

12 JUN 85

12 JUN85

18.JUN85

18JUN85

18JUN85

25JUN85

25JUN 85

25JUN85

02JUL8 5

02JUL85

02JUL85

11JUL8 5

11JUL85

11JUL85

12JUL85

12JUL85

12JUL85

13JUL85

13JUL85

13JUL85

14JUL85

14JUL85

15JUL85

15JUL85

15 JUL85

16JUL85

16JUL85

16JUL85

17JUL85

17JUL85

17JUL8 5

18JUL85

18JUL85

\section{DEPTH FROM}

SURFACE $(\mathrm{cm}$.

445

445

445

445

445

445

445

445

445

445

445

445.

445

445

445

445

445

445

445

445

445

445

445

445

445

445

445

445

445

445

445

445

445

445

445

445

445

445

445

445

445

445

445

445

445

$\begin{array}{ll}\text { DISTANCE } & \text { VOLUMETRIC } \\ \text { FROM } & \text { MOISTURE } \\ \text { CENTER } & \text { CONTENT }(\%)\end{array}$

60
120

23.7

21.6

25.0

23.8

19.8

24.9

23.1

20.0

24.7

23.1

19.4

24.1

22.9

19.5

24.5

23.1

19.5

24.4

22.9

19.2

24.3

23.0

19.5

24.1

22.7

18.9

22.9

22.2

18.7

23.4

21.7

17.4

23.2

21.9

23.5

21.7

13.4

22.8

21.7

12.3

23.3

22.2

18.3

23.1 


SAMPLING
DATE

18JUL85

$19 J U L 85$

19JUL85

19JUL85

21JUL85

21JUL85

21JUL85

22JUL85

22JUL85

22 JUL85

24JUL85

24JUL85

24JUL85

25JUL85

25JUL85

25JUL85

26JUL 85

26JUL85

26JUL85

27JUL85

27 JUL85

27 JUL85

28JUL85

28JUL85

28JUL85

29JUL85

29 JUL85

29JUL85

30JUL85

30 JUL85

30 JUL85

O2AUG 85

$02 A U G 85$

02AUG85

04AUG85

04AUG 85

04AUG85

06AUG85

06AUG85

06AUG85

09AUG85

09AUG85

09AUG85

14AUG85

14AUG85
DEPTH FROM

SURFACE $(\mathrm{cm}$.

445

445

445

445

445

445

445

445

445

445

445

445

445

445

445

445

445

445

445

445

445

445

445

445

445

445

445

445

445

445

445

445

445

445

445

445

445

445

445

445

445

445

445

445

445

$\begin{array}{ll}\text { DISTANCE } & \text { VOLUMETRIC } \\ \text { FROM } & \text { MOISTURE } \\ \text { CENTER } & \text { CONTENT }(\xi)\end{array}$

120

18.3

22.4

21.9

11.0

24.4

22.8

18.7

26.4

25.0

22.4

28.3

26.4

25.0

27.9

26.4

24.8

28.0

26.1

25.6

28.5

26.4

25.6

28.0

26.8

26.4

28.2

26.4

26.1

28.2

26.6

24.9

28.9

26.5

25.7

28.6

26.7

25.7

28.9

26.7

26.0

28.9

26.6

25.9

28.5

26.6 
SAMPIING

DATE

14AUG85

16AUG85

16 AUG85

16AUG85

21 AUG 85

21AUG85

21 AUG 85

23AUG85

23AUG85

23AUG85

27 AUG85

27 AUG85

27 AUG85

$30 A U G 85$

$30 A U G 85$

30AUG8 5

03SEP8 5

03SEP85

$03 S E P 85$

09SEP85

09SEP85

09SEP85

$18 \$ E P 85$

$18 S E P 85$

18 SEP85

$30 S E P 85$

$30 S E P 85$

30 SEP 85

090CT85

$090 \mathrm{CT} 85$

$090 \mathrm{CT} 85$

$160 \mathrm{CT} 85$

$160 \mathrm{CT} 85$

$160 \mathrm{CT} 85$

$230 \mathrm{CT} 85$

$230 \mathrm{CT} 85$

$230 \mathrm{CT} 85$

$300 \mathrm{CT} 85$

$300 \mathrm{CT} 85$

$300 \mathrm{CT} 85$

06NOV85

06 NOV 85

06NOV 85

\section{DEPTH FROM}

SURFACE (cm.)

445

445

445

445

445

445

445

445

445

445

445

445

445

445

445

445

445

445

445

445

445

445

445

445

445

445

445

445

445

445

445

445

$+45$

445

445

445

445

445

445

445

445

445

445

$\begin{array}{ll}\text { DISTANCE } & \text { VOLUMETRIC } \\ \text { FROM } & \text { MOISTURE } \\ \text { CENTER } & \text { CONTENT ( } 8)\end{array}$

24.9

28.6

26.9

25.4

29.2

26.7

25.9

28.4

26.6

26.3

28.8

26.9

26.3

29.0

26.6

26.3

28.9

2.6 .7

25.9

29.1

27.0

26.2

29.1

27.3

26,6

28.7

27.1

25.8

28.6

27.1

25.9

28.7

26.7

25.9

29.4

26.7

25.5

28.8

26.8

24.0

28.2

26.1

25.3 


\section{APPENDIX B}

TRACER DATA BASE

FOR THE CAISSON B EXPERIMENTS 


\section{FOR THE CAISSON B EXPERIMENTS}

This data base contains the iodide, bromide, and lithium concentration data for the soil solutions collected in Caisson B as a function of sampling depth and time for several pulse experiments. The data presented are for the samples collected $1.5 \mathrm{~m}$ into the tuff matrix from the six access ports within Caisson $B$, as well as the vertical samples and the caisson outflow samples. 


\begin{tabular}{|c|c|c|c|}
\hline & $\begin{array}{l}\text { Iodide } \\
\text { Concentration }\end{array}$ & Bromide & $\begin{array}{l}\text { Lithium } \\
\text { Concentration }\end{array}$ \\
\hline & $(\mathrm{ppm})$ & (ppm) & (ppm) \\
\hline
\end{tabular}

\section{AUG84}

05SEP84

07SEP84

$115 E P 84$

$12 S E P 84$

$13 S E P 84$

14 SEP84

17SEP84

19SEP 84

20SEP 84

21SEP84

24SEP 84

25SEP 84

26SEP84

27SEP84

28SEP84

$010 \mathrm{CT} 84$

$020 \mathrm{CT} 84$

$030 \mathrm{CT} 84$

040CT 84

050CT 84

090CT84

100CT84

$110 \mathrm{CT} 84$

$120 \mathrm{CT} 84$

$150 \mathrm{CT} 84$

$160 \mathrm{CT} 84$

$170 \mathrm{CT} 84$

$180 \mathrm{CT} 84$

$190 \mathrm{CT} 84$

$220 \mathrm{CT} 84$

$230 \mathrm{CT} 84$

240CT 84

$250 \mathrm{CT} 84$

260CT 84

290CT 84

$300 \mathrm{TT} 84$

$310 \mathrm{CT} 84$

02NoV84

05NOV84

07 NOV 84

09NOV 84

12 NOV 84

14 NOV 84

16 NOV84

$$
0.03
$$

0.03

0.03

0.03

0.03

0.03

0.02

0.04

2.60

12.40

64.00

130.00

150.00

6.30

0.56

0.35

0.44

0.47

2.00

2. 10

1.70

1.40

1.30

0.33

0.64

0.76

1.40

0.23

0.09

0.04

1.10

0.10

0.35

0.38

0.22

0.09

0.03

0.02

0.06

0.05
0.020

0.020

0.020

0.020

0.020

0.020

0.020

0.041

1. 520

1. 502

6.880

33.730

67.470

82.190

2. 600

0.105

0.113

0.297

0.377

1.670

1.670

1.418

1. 283

1.040

0.258

0.459

0.499

0.870

0.102

0.076

0.057

0.052

0.131

0.378

0.309

0.139

0.020

0.020

0.020

0.020

0.020
0.037

0.040

0.080

0.080

0.070

0.660

3.690

5. 330

0.370

0.070

0.050

0.100

0.140

0.320

0.340

0.340

0.300

0.210

0.070

0.060

0.120

0.130

0.050

0.030

0.040

0.050

0.030 


$\begin{array}{llll} & \text { Iodide } & \text { Bromide } & \text { Lithium } \\ \text { Sampling } & \text { Concentration } & \text { Concentration } & \text { Concentration } \\ \text { Date } & \text { (ppm) } & \text { (ppm) } & \text { (ppm) }\end{array}$

\begin{tabular}{|c|c|c|c|}
\hline 21 NOV84 & 0.04 & & . \\
\hline 23NOV84 & 0.03 & 0.109 & . \\
\hline 26 NOV84 & . & 0.726 & . \\
\hline 28NOV84 & 0.03 & 0.020 & . \\
\hline 30NOV84 & . & 0.127 & . \\
\hline 03DEC84 & 0.04 & 0.364 & . \\
\hline 05DEC84 & 0.02 & 0.389 & $\cdot$ \\
\hline 07DEC84 & 0.43 & 0.402 & 0.04 \\
\hline 08DEC84 & 58.00 & 28.135 & 2.38 \\
\hline 09DEC84 & 146.00 & 67.880 & 5.41 \\
\hline 10DEC84 & 130.00 & 77.200 & 6.32 \\
\hline 11DEC84 & 160.00 & 81.630 & 7.87 \\
\hline $12 \mathrm{DEC} 84$ & 159.00 & 87.180 & 8.02 \\
\hline 13DEC84 & 152.00 & 78.610 & 7.97 \\
\hline 14DEC84 & 102.00 & 51.430 & 5.55 \\
\hline 15DEC84 & 38.00 & 19.670 & 2.65 \\
\hline 17DEC84 & 7.20 & 3.540 & 0.59 \\
\hline 18DEC84 & 2.85 & 1.445 & 0.20 \\
\hline 19DEC84 & 1.27 & 0.769 & 0.10 \\
\hline 20DEC84 & . & 0.272 & . \\
\hline 20DEC84 & 0.61 & $\cdot$ & 0.06 \\
\hline 21DEC84 & . & 0.290 & . \\
\hline 21DEC84 & 0.48 & . & 0.06 \\
\hline 22DEC84 & . & 0.184 & . \\
\hline 22DEC 84 & 0.36 & & 0.05 \\
\hline 24DEC84 & & 0.165 & . \\
\hline 24DEC84 & 0.33 & . & 0.04 \\
\hline 26DEC84 & . & 0.006 & . \\
\hline 26DEC84 & 0.22 & $\cdot$ & 0.04 \\
\hline 27DEC84 & . & 0.274 & . \\
\hline 27DEC 84 & 0.58 & . & 0.09 \\
\hline 28DEC 84 & . & 0.020 & . \\
\hline 28DEC84 & 0.13 & . & 0.04 \\
\hline 29DEC84 & 0.11 & . & 0.04 \\
\hline 31 DEC84 & 0.11 & . & 0.04 \\
\hline 02JAN85 & 0.10 & . & 0.04 \\
\hline 03JAN85 & 0.12 & . & . \\
\hline 04JAN85 & 0.10 & . & . \\
\hline 05JAN85 & . & 0.067 & . \\
\hline 05JAN85 & 0.10 & . & . \\
\hline 07JAN85 & 0.06 & . & . \\
\hline O8JAN85 & 0.05 & . & . \\
\hline 09JAN85 & 0.49 & . & . \\
\hline 10JAN85 & 0.05 & . & . \\
\hline 11JAN85 & 0.05 & . & . \\
\hline
\end{tabular}




\begin{tabular}{|c|c|c|c|}
\hline & Iodide & Bromide & Lithium \\
\hline & $(\mathrm{ppm})$ & (ppm) & (ppm) \\
\hline
\end{tabular}

\begin{tabular}{|c|c|}
\hline 12JAN85 & 0.060 \\
\hline 14JAN85 & 0.070 \\
\hline 16JAN85 & 0.030 \\
\hline 18JAN85 & 0.030 \\
\hline 18JAN85 & 0.050 \\
\hline 21JAN85 & 0.049 \\
\hline 25JAN85 & 0.040 \\
\hline 28JAN85 & \\
\hline 28JAN85 & 0.040 \\
\hline 30JAN85 & 0.040 \\
\hline 01 FEB 85 & . \\
\hline 01FEB85 & 0.040 \\
\hline 04FEB 85 & . \\
\hline 04FEB85 & 0.020 \\
\hline 06FEB85 & \\
\hline 06FEB85 & 0.020 \\
\hline 08FEB85 & · \\
\hline O8FEB 85 & 0.020 \\
\hline 09FEB85 & 3.460 \\
\hline 10FEB85 & 98.000 \\
\hline $11 \mathrm{FEB} 85$ & \\
\hline 11 FEB85 & 177.000 \\
\hline 12 FEB85 & \\
\hline 12FEB85 & 198.000 \\
\hline 13 FEB 85 & \\
\hline $13 \mathrm{FEB} 85$ & 156.000 \\
\hline 14FEB85 & \\
\hline 14FEB85 & 143.000 \\
\hline 15FEB8 5 & 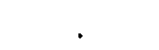 \\
\hline $15 \mathrm{FEB} 85$ & 140.000 \\
\hline 16FEB85 & 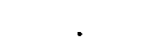 \\
\hline 16FEB8 5 & 107.000 \\
\hline $18 \mathrm{FEB} 85$ & \\
\hline $18 \mathrm{FEB} 85$ & 28.500 \\
\hline 19FEB85 & $\cdot$ \\
\hline 19FEB85 & 10.100 \\
\hline 20FEB 85 & \\
\hline 20FEB85 & 3.300 \\
\hline 21FEB85 & \\
\hline 21FEB85 & 1.550 \\
\hline 22FEB85 & \\
\hline 22FEB85 & 0.950 \\
\hline 23FEB85 & 0.400 \\
\hline 25FEB85 & 0.230 \\
\hline
\end{tabular}




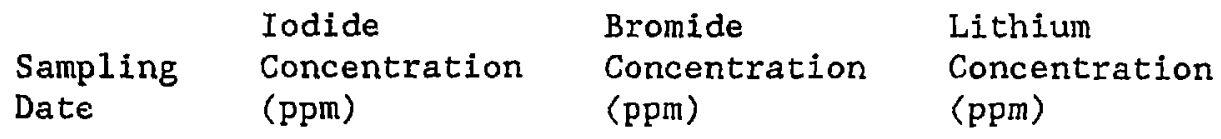

27FEB85

28FEB 85

01MAR 85

02MAR8 5

04MAR85

05MAR 85

OTMAR85

O8MAR85

10MAR85

11MAR85

13MAR85

15MAR85

18MAR85

20MAR 85

22MAR85

25MAR85

27MAR85

09APR85

09APR85

10APR 85

10APR 85

11APR85

11APR 85

12APR 85

12APR85

13AFR8 5

13APR 85

14APR 85

14APR8 5

15APR 85

15APR 85

16APR85

16APR85

17APR 85

17APR 85

18APR 85

18APR 85

19APR85

19APR85

20APR85

21APR 85

21APR 85

22APR 85

23APR 85

24APR85
0.13

0.26

0.36

0.22

1.47

0.19

0.24

0.27

0.17

0.27

0.14

0.43

0.54

0.07

0.05

0.06

0.05

14.40

109.00

164.00

188.00

178.00

174.00

113.00

28.00

5.60

1. 23

0.57

.

0.25

0.18

0.17

0.21 $\begin{array}{ll}\text { Bromide } & \text { Lithium } \\ \text { Concentration } & \text { Concentration } \\ \text { (ppm) } & (\mathrm{ppm})\end{array}$

9.17

67.60

91.20

0.29

4. 31

6.83

103.00

7.68

94.10

7.54

102.00

7.88

66.20

15.80

6.01

1.80

2.63

0.28

0.43

0.12

0.05

0.06

0.25

0.11

.

0.04

0.04 


\begin{tabular}{|c|c|c|c|}
\hline $\begin{array}{l}\text { Sampling } \\
\text { Date }\end{array}$ & $\begin{array}{l}\text { Iodide } \\
\text { Concentration } \\
\text { (ppm) }\end{array}$ & $\begin{array}{l}\text { Bromide } \\
\text { Concentration } \\
\text { (ppm) }\end{array}$ & $\begin{array}{l}\text { Lithium } \\
\text { Concentration } \\
\text { (ppm) }\end{array}$ \\
\hline 25APR 85 & 0.16 & . & . \\
\hline 26APR 85 & 0.17 & . & 0.04 \\
\hline 28APR85 & 0.16 & . & 0.04 \\
\hline 29APR85 & 0.06 & . & . \\
\hline 02MAY85 & 0.11 & . & . \\
\hline 08MAY85 & 0.17 & . & . \\
\hline 15MAY85 & 0.23 & . & . \\
\hline 13JUL85 & 125.00 & . & 4.45 \\
\hline 14JUL85 & 171.00 & . & 7.16 \\
\hline 15JUL85 & 155.00 & . & 7.16 \\
\hline 16JUL85 & 186.00 & . & 8.29 \\
\hline 17JUL85 & 185.00 & . & 8.42 \\
\hline 18JUL85 & 145.00 & . & 7.52 \\
\hline 19JUL85 & 196.00 & . & 8.46 \\
\hline 20JUL85 & 123.00 & . & 5.71 \\
\hline 21JUL85 & 27.00 & . & 1.81 \\
\hline 22JUL85 & 7.60 & . & 0.61 \\
\hline 23JUL85 & 1.37 & . & 0.16 \\
\hline 24JUL85 & 1.62 & . & 0.14 \\
\hline 25JUL85 & 6.20 & . & 0.36 \\
\hline 26JUL85 & 49.00 & . & 0.32 \\
\hline 27JUL85 & . & . & 0.13 \\
\hline 28JUL8 5 & . & . & 0.07 \\
\hline 29JUL85 & . & . & 0.12 \\
\hline
\end{tabular}




\begin{tabular}{|c|c|c|c|}
\hline $\begin{array}{l}\text { Sampling } \\
\text { Date }\end{array}$ & $\begin{array}{l}\text { Iodide } \\
\text { Concentration } \\
\text { (ppm) }\end{array}$ & $\begin{array}{l}\text { Bromide } \\
\text { Concentration } \\
\text { (ppm) }\end{array}$ & $\begin{array}{l}\text { Lithium } \\
\text { Concentration } \\
\text { (ppm) }\end{array}$ \\
\hline 17AUG84 & . & . & 0.030 \\
\hline 18AUG84 & . & . & 0.030 \\
\hline 19AUG 84 & . & . & 0.030 \\
\hline 20AUG84 & . & . & 0.030 \\
\hline 21AUG84 & . & . & 0.030 \\
\hline 31 AUG84 & . & 0.020 & . \\
\hline 06SEP84 & 0.03 & · & . \\
\hline 07SEP84 & . & 0.020 & 0.030 \\
\hline 12SEP84 & . & 0.020 & . \\
\hline 13 SEP84 & 0.04 & 0.020 & . \\
\hline 14SEP84 & 0.04 & 0.020 & 0.452 \\
\hline 17SEP84 & 0.04 & 0.020 & . \\
\hline 19SEP84 & 0.03 & 0.020 & . \\
\hline 20SEP84 & 0.01 & 0.020 & 0.040 \\
\hline 21SEP84 & 0.01 & 0.020 & 0.040 \\
\hline 24SEP84 & 0.01 & 0.020 & 0.040 \\
\hline 25SEP84 & 0.01 & 0.020 & 0.040 \\
\hline 26SEP84 & 0.01 & 0.020 & 0.040 \\
\hline 27SEP84 & 0.02 & 0.075 & 0.040 \\
\hline 28SEP84 & 0,02 & 0.020 & 0.040 \\
\hline 010CT84 & 0.03 & 0.020 & 0.040 \\
\hline 020СТ84 & 0.48 & 0.305 & 0.040 \\
\hline $030 \mathrm{CT} 84$ & 6.00 & 3.475 & 0.040 \\
\hline $040 C T 84$ & 42.00 & 24.300 & 0.030 \\
\hline $050 C T 84$ & 73.00 & 53.610 & 0.560 \\
\hline 090СT84 & 100.00 & 70.400 & 5.410 \\
\hline $100 \mathrm{CT} 84$ & 107.00 & 69.100 & 7.490 \\
\hline $110 \mathrm{CT} 84$ & 101.00 & 63.680 & 8.180 \\
\hline $120 \mathrm{CT} 84$ & 94.00 & 56.820 & 8.410 \\
\hline $150 \mathrm{CT} 84$ & 40.00 & 28.285 & 6.490 \\
\hline $160 \mathrm{CT} 84$ & 21.00 & 12.111 & 3.250 \\
\hline $170 C T 84$ & 16.00 & 7.869 & 1.990 \\
\hline $180 \mathrm{CT} 84$ & 10.70 & 5.372 & 1.470 \\
\hline $190 \mathrm{CT} 84$ & 7.00 & 2.976 & 1.090 \\
\hline $220 \mathrm{CT} 84$ & 3.10 & 1.268 & 0.560 \\
\hline $230 \mathrm{CT} 84$ & 1.46 & 0.566 & 0.280 \\
\hline $240 \mathrm{CT} 84$ & 1.10 & 0.371 & 0.200 \\
\hline $250 \mathrm{CT} 84$ & 0.73 & 0.240 & 0.150 \\
\hline $260 \mathrm{CT} 84$ & 0.65 & 0.197 & 0.120 \\
\hline $290 C T 84$ & 0.85 & 0.339 & \\
\hline $300 C T 84$ & 0.01 & 0.160 & 0.050 \\
\hline $310 \mathrm{CT} 84$ & 0.01 & 0.020 & 0.060 \\
\hline 02NOV84 & 0.01 & 0.020 & 0.060 \\
\hline 05NOV84 & 0.05 & 0.020 & 0.050 \\
\hline 07 NOV84 & 0.01 & 0.020 & 0.050 \\
\hline
\end{tabular}


Sampling

Date

09Nov84

12 NOV 84

$14 N 0 V 84$

16NOV 84

19NOV84

21 NOV 84

23NOV 84

26NOV84

28NOV84

30 NOV 84

03DEC 84

05DEC84

07DEC 84

08DEC84

09DEC84

10DEC84

11DEC84

12DEC84

13DEC84

14DEC84

15DEC84

17DEC84

18DEC84

19DEC84

20DEC 84

20DEC 84

21DEC 84

21DEC 84

22DEC 84

22DEC 84

24DEC 84

24DEC 84

26DEC 84

26DEC 84

27DEC84

27DEC84

28DEC84

28 DEC 84

29 DEC 84

29DEC84

31DEC 84

31 DEC 84

02JAN8 5

02JAN85

03JAN85

\section{Iodide}

Concentration

(ppm)

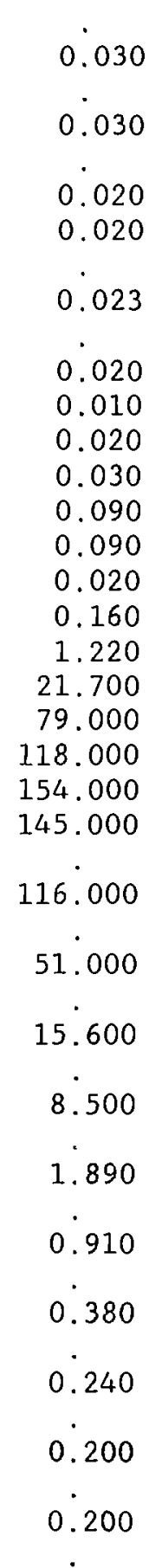

Bromide

Concentration

(ppm)

0.020
0.020

Lithium

Concentration

(ppm)

$$
\begin{aligned}
& 0.06 \\
& 0.06 \\
& 0.06 \\
& 0.07 \\
& 0.08 \\
& 0.05 \\
& 0.05 \\
& . \\
& . \\
& . \\
& . \\
& . \\
& . \\
& . \\
& . \\
& 0.04 \\
& 0.04
\end{aligned}
$$

0.020

0.020

0.497

0.150

0.293

0.227

0.435

0.329

0.299

0.520

0.514

0.020

0.620

10.840

42.970

69.190

84.550

75.830

88.520

25.550

7.870

4. 239

0.08

0.08

0.51

3.44

5.35

6.92

7.11

5.80

3.40

0.699

1.70

0.67

0.236

0.150

0.087

0.648

0.168

0.020

0.21

.

.

0.13 


$\begin{array}{llll} & \text { Iodide } & \text { Bromide } & \text { Lithium } \\ \text { Sampling } & \text { Concentration } & \text { Concentration } & \text { Concentration } \\ \text { Date } & \text { (ppm) } & \text { (ppm) } & \text { (ppm) }\end{array}$

\begin{tabular}{|c|c|c|c|}
\hline 03JAN85 & 0.13 & - & $\circ \quad$ \\
\hline 04JAN85 & 0.12 & . & 0.08 \\
\hline 05JAN85 & . & 0.270 & . \\
\hline 05JAN85 & 0.10 & & 0.07 \\
\hline 07JAN85 & . & 0.020 & . \\
\hline 07JAN85 & 0.08 & . & 0.07 \\
\hline 08JAN85 & 0.07 & . & . \\
\hline O9JAN85 & . & 0.020 & . \\
\hline O9JAN85 & 0.06 & • & . \\
\hline 10JAN85 & 0.06 & . & . \\
\hline 11JAN85 & 0.06 & . & . \\
\hline 12JAN85 & 0.05 & . & . \\
\hline 14J AN85 & . & 0.020 & . \\
\hline 14JAN85 & 0.07 & . & . \\
\hline 16JAN85 & 0.05 & . & . \\
\hline 18JAN85 & 0.02 & . & . \\
\hline 21JAN85 & . & 0.060 & . \\
\hline 21JAN85 & 0.12 & . & . \\
\hline 23JAN85 & 0.02 & . & . \\
\hline 25JAN85 & 0.07 & . & . \\
\hline 28JAN85 & · & 0.010 & . \\
\hline 28JAN85 & 0.07 & . & . \\
\hline 30JAN85 & 0.06 & . & . \\
\hline 01FEB85 & 0.06 & 0.020 & . \\
\hline 04FEB85 & 0.37 & 0.255 & . \\
\hline $06 \mathrm{FEB} 85$ & 0.03 & 0.427 & . \\
\hline 08 FEB 85 & 0.02 & 0.061 & . \\
\hline 09FEB85 & 0.02 & . & . \\
\hline 10FEB85 & 0.02 & . & . \\
\hline $11 F E B 85$ & 0.06 & • & . \\
\hline 12 FEB 85 & 0.05 & 0.020 & . \\
\hline 13 FEB 85 & 0,02 & $\cdot$ & . \\
\hline $14 \mathrm{FEB} 85$ & 0.02 & 0.110 & . \\
\hline 15FEB85 & 5.44 & 2.968 & 0.04 \\
\hline 16FEB85 & 37.30 & 22.220 & 0.06 \\
\hline $18 \mathrm{FEB} 85$ & 122.00 & 65.000 & 1.73 \\
\hline 19FEB85 & 150.00 & . & 4.52 \\
\hline $20 \mathrm{FEB} 85$ & 157.00 & 80,000 & 5.47 \\
\hline 21 FEB8 5 & 155.00 & 71.000 & 5.70 \\
\hline 22 FEB 85 & 121.00 & 66.000 & 5.70 \\
\hline $23 F E B 85$ & 73.10 & 46.000 & 5.37 \\
\hline 25FEB85 & 32.90 & 20.000 & 3.65 \\
\hline 26FEB85 & 14.80 & 9.000 & 1.83 \\
\hline 27FEB85 & 9.00 & 5.000 & 1.19 \\
\hline $28 \mathrm{FEB} 85$ & 8.36 & 3.000 & 0.82 \\
\hline
\end{tabular}




$\begin{array}{llll} & \text { Iodide } & \text { Bromide } & \text { Lithium } \\ \text { Sampling } & \text { Concentration } & \begin{array}{l}\text { Concentration } \\ \text { (ppm) }\end{array} & \begin{array}{l}\text { Concentration } \\ \text { Date }\end{array} \\ \text { (ppm) } & \text { (ppm) }\end{array}$

OLMAR 85

O2MAR 85

04MAR 85

O5MAR 85

O6MAR 85

07MAR8 5

O8MAR 85

11 MAR 85

13 MAR 85

15MAR 85

18MAR 85

20MAR 85

22MAR 85

25MAR 85

27MAR8 5

13APR 85

14APR85

14 APR 85

15APR 85

$15 A P R 85$

16APR85

16APR85

17APR 85

17APR 85

18APR 85

18APR 85

19APR8 5

19APR 85

20APR 85

21APR 85

21 APR 85

22APR 85

22APR 85

23APR 85

23APR 85

24APR 85

24APR 85

25APR 85

25APR 85

26APR 85

26APR 85

28APR 85

29APR 85

02 MAY 85

08MAY85
6.16

5.12

3. 72

2. 65

1.47

0.90

0.49

0.23

0.09

0.29

0.43

0.15

0.11

0.12

0.09

0.32

9.90

51.00

106.00

155.00

169.00

131.00

65.00

23.00

6.60

3.10

1. 36

0.90

0.47

0.44

0.40
3.00

0.30

1.00

0.90

0.70

1.00

1.00

0.24

6.22

31.10

60.30

80.90

88.80

91.00

72.40

36.30

11.90

3.29

1.15

0.44

0.59

0.47

0.33

.

.

.

.

0.04

0.04

0.04

0.17

1. 38

3.73

5. 72

6.51

5.62

3. 26

1.94

1. 22

0.66

0.36

0.20

0.15

0.12 
Sampling

Date

15MAY85

15JUL85

16JUL85

17JUL85

18JUL85

19JUL85

20JUL85

21JUL85

22JUL85

23JUL85

24 JUL85

25JUL85

26JUL85

27JUL85

28.UL85

29JUL85

30 JUL85

OIAUG85

02AUG85

04AUG85

\section{Bromide}

Concentration

(ppm)
Lithium

Concentration

(ppm)

$$
\begin{array}{r}
0.41 \\
2.90 \\
1.35 \\
1.90 \\
13.40 \\
69.00 \\
150.00 \\
170.00 \\
170.00 \\
170.00 \\
141.00 \\
96.00 \\
31.70
\end{array}
$$

013

0.19

0.12

0.42

2.47

5.79

7.17

7.59

7.46

7.32

5.92

3.28

1.66

0.93

0.52

0.31

0.18

0.13 


$\begin{array}{llll} & \text { Iodide } & \text { Bromide } & \text { Lithium } \\ \text { Sampling } & \text { Concentration } & \text { Concentration } & \text { Concentration } \\ \text { Date } & \text { (ppm) } & \text { (ppm) } & \text { (ppm) }\end{array}$

17AUG84

18AUG84

19AUG84

20AUG 84

21AUG84

30 AUG 84

04SEP84

O6SEP84

11SEP 84

13 SEP 84

14 SEP 84

17 SEP 4

19SEP84

20SEP 84

21SEP 84

24SEP 84

25SEP 84

26SEP84

27SEP 84

28SEP 84

$010 C \Gamma 84$

$020 \mathrm{CT} 84$

$030 \mathrm{CT} 84$

$040 \mathrm{CT} 84$

050CT 84

$090 \mathrm{CT} 84$

$100 \mathrm{CT} 84$

$110 \mathrm{CT} 84$

$120 \mathrm{CT} 84$

$150 \mathrm{CT} 84$

160 TT 84

$170 \mathrm{CT} 84$

180CT 84

$190 \mathrm{CT} 84$

$220 \mathrm{CT} 84$

$230 \mathrm{CT} 84$

240CT 84

$250 \mathrm{CT} 84$

$260 \mathrm{CT} 84$

290CT 84

$300 \mathrm{CT} 84$

$310 \mathrm{CT} 84$

02NOV84

05NOV84

07NOV84

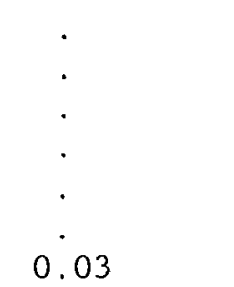

.

•

0.03

0.02

0.01

0.05

0.02

0.02

0.25

0.09

0.11

0.01

0.01

0.01

0.01

0.01

0.09

0.95

4.10

10.70

94.00

129.00

113.00

102.00

82.00

12.30

1.46

0.64

0.38

0.12

0.12

0.04

0.03

0.05

0.04

0.03
0.020

0.020

0.020

0.020

0.020

0.020

0.096

0.020

0.020

0.020

0.770

0.090

0.020

0.020

0.205

0.020

0.020

0.020

0.067

0.028

0.020

0.089

0.656

2.837

7.402

60.900

77.490

66.710

60.350

53.185

7.422

0.736

0.380

0.187

0.020

0.020

0.020

0.020

0.020

0.020

0.020
.

0.030

.

.

.

.

.

.$$
\text { . }
$$

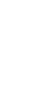$$
\text { . }
$$$$
\text { . }
$$$$
\text { . }
$$$$
\text { . }
$$$$
0.030
$$

0.030

0.030

0.030

0.030

0.060

0.040

3.980

6.240

7.070

2. 490

1. 220

0.800

0.520

0.350

0.270

0.225

0.200

0.150

0.110

0.100 


$\begin{array}{llll} & \text { Iodide } & \text { Bromide } & \text { Lithium } \\ \text { Sampling } & \text { Concentration } & \begin{array}{l}\text { Concentration } \\ \text { (ppm) }\end{array} & \begin{array}{l}\text { Concentration } \\ \text { Date }\end{array} \\ \text { (ppm) } & \text { (ppm) }\end{array}$

09Nov84

$\begin{array}{lc}0.070 & 0.200 \\ 0.080 & 0.020 \\ 0.070 & 0.155 \\ 0.060 & . \\ 0.040 & 0.020 \\ 0.030 & 0.047 \\ 0.033 & 0.020 \\ 0.030 & 0.544 \\ 0.050 & 0.198 \\ 0.030 & 0.327 \\ 0.020 & 0.227 \\ 0.020 & 0.220 \\ 0.050 & 0.157 \\ 0.020 & 0.231 \\ 0.030 & 0.020 \\ 0.030 & 0.658 \\ 0.050 & 0.598 \\ 0.040 & 0.020 \\ 0.220 & 0.020 \\ 0.140 & 0.098 \\ 0.100 & 0.077 \\ 0.100 & 0.020 \\ 0.090 & 0.020 \\ 4.900 & 2.274 \\ 54.000 & \end{array}$

12 NOV84

14 NOV 84

16 NOV84

19 NOV 84

21 NOV 84

23 NOV 84

26 NOV84

28 NOV 84

30 NOV 84

03DEC84

$05 \mathrm{DEC} 84$

$07 D E C 84$

$07 D E C 84$

08DEC84

09DEC 84

10DEC84

11DEC84

12DEC 84

13DEC 84

14DEC84

15DEC84

17 DEC 84

$18 D E C 84$

19DEC84

20DEC84

21 DEC84

22DEC84

24DEC 84

26DEC84

27 DEC84

$28 \mathrm{DEC} 84$

29DEC 84

31DEC84

02JAN85

03JAN85

04J AN85

O5JAN85

07JAN85

08JAN85

09JAN85

10JAN8 5

11JAN85

12JAN85

14JAN85

54.000

114.000

133.000

136.000

71.000

26.500

10.100

5.200

1.850

0.970

0.690

0.500

0.400

0.270

0.230

0.210

0.180

0.170

0.130

0.120

0.09

\section{Bromide \\ Concentracion}

0.200

0.09

0.07

0.07

0.06

0.06

0.06 


\begin{tabular}{|c|c|c|c|}
\hline $\begin{array}{l}\text { Sampling } \\
\text { Date }\end{array}$ & $\begin{array}{l}\text { Iodide } \\
\text { Concentration } \\
\text { (ppm) }\end{array}$ & $\begin{array}{l}\text { Bromide } \\
\text { Concentration } \\
\text { (ppm) }\end{array}$ & $\begin{array}{l}\text { Lithium } \\
\text { Concentration } \\
\text { (ppm) }\end{array}$ \\
\hline 16JAN85 & 0.09 & . & . \\
\hline 18JAN85 & 0.02 & . & . \\
\hline 21JAN85 & 0.10 & . & . \\
\hline 23JAN85 & 0.02 & . & . \\
\hline 25JAN85 & 0.09 & . & . \\
\hline 28JAN85 & 0.10 & . & . \\
\hline 30JAN85 & 0.09 & & . \\
\hline 01FEB85 & 0.07 & 0.125 & . \\
\hline 04FEB85 & 0.03 & 0.094 & . \\
\hline 06FEB85 & 0.03 & 0.514 & . \\
\hline 08 FEB 85 & 0.04 & 0.083 & . \\
\hline 09FEB85 & 0.04 & . & . \\
\hline 10FEB85 & 0.03 & . & . \\
\hline 11 FEB85 & 0.05 & & . \\
\hline 12FEB8 5 & 0.04 & 0.020 & . \\
\hline $13 \mathrm{FEB} 85$ & 0.02 & & . \\
\hline $14 \mathrm{FEB} 85$ & 0.02 & 0.020 & . \\
\hline 15FEB 85 & 0.01 & & . \\
\hline 16FEB85 & 0.05 & 0.020 & . \\
\hline 18FEB85 & 0.04 & 0.400 & . \\
\hline 19FEB85 & 0.08 & . & . \\
\hline 20FEB85 & 0.07 & & . \\
\hline $21 \mathrm{FEB} 85$ & 4.80 & 1.000 & 0.04 \\
\hline 22FEB 85 & 37.20 & 17.000 & 0.04 \\
\hline 23 FEB8 5 & 89.00 & 47.000 & 0.05 \\
\hline 25FEB85 & 124.00 & 81.000 & 2.81 \\
\hline 26FEB85 & 139.00 & 80.000 & 4.66 \\
\hline 27 FEB85 5 & 116.00 & 78.000 & 5.29 \\
\hline $28 \mathrm{FEB} 85$ & 120.00 & 66.000 & 5.66 \\
\hline O1MAR85 & 73.10 & 43.000 & 5.65 \\
\hline 02MAR85 & 42.30 & 23.000 & 5.07 \\
\hline 04MAR85 & 20.10 & 7.000 & 3.26 \\
\hline 05MAR85 & 9.50 & 4.000 & 2.27 \\
\hline 06MAR8 5 & 6.00 & 3.000 & 1.58 \\
\hline 07MAR 85 & 4.00 & 2.000 & 1.10 \\
\hline 08MAR85 & 2.24 & 2.000 & 0.82 \\
\hline 11MAR85 & 1.60 & . & 0.50 \\
\hline 13MAR85 & 0.50 & . & . \\
\hline 15MAR 85 & 0.08 & . & . \\
\hline 18MAR85 & 0.40 & . & . \\
\hline 20MAR85 & 0.14 & . & . \\
\hline 22MAR85 & 0.77 & . & . \\
\hline 25MAR85 & 0.17 & . & . \\
\hline 27MAR85 & 0.11 & . & . \\
\hline 29MAR85 & 0.10 & . & . \\
\hline
\end{tabular}




$\begin{array}{ll} & \text { Iodide } \\ \text { Sampling } & \text { Concentration } \\ \text { Date } & \text { (ppm) }\end{array}$

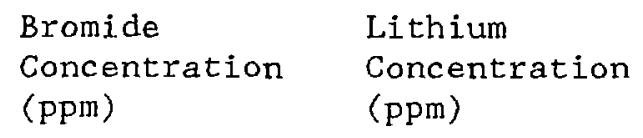

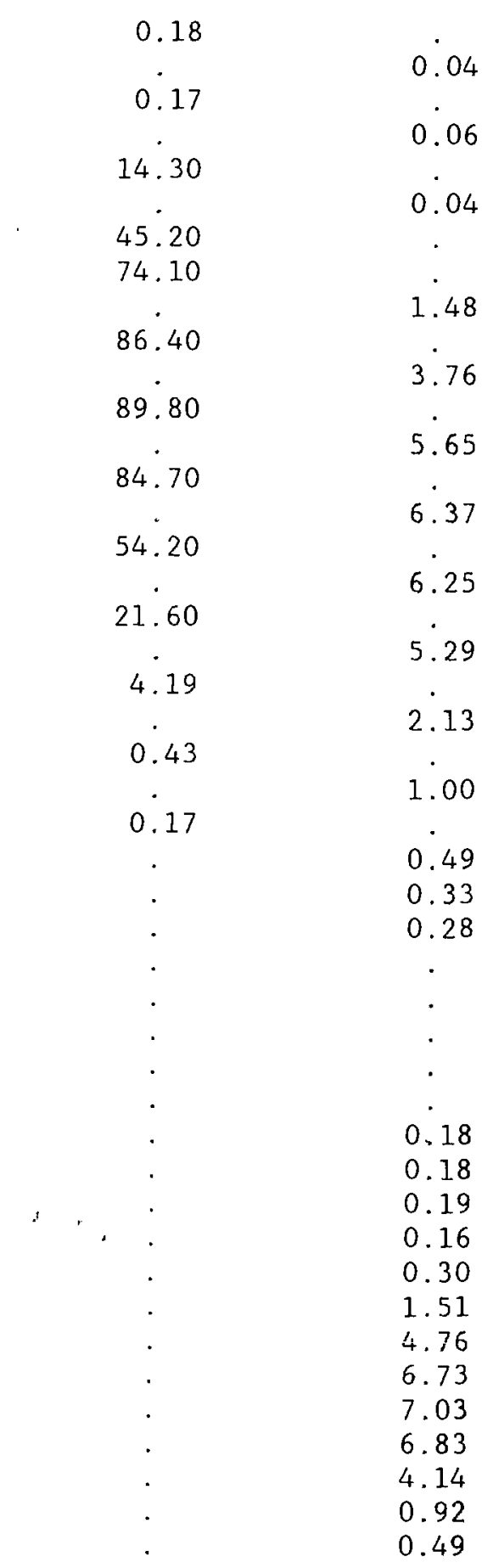




$\begin{array}{ll} & \text { Ioride } \\ \text { Sampling } & \text { Concentration } \\ \text { Date } & \text { (ppm) }\end{array}$

$\begin{array}{ll}\text { Bromide } & \text { Lithium } \\ \text { Concentration } & \text { Concentration } \\ \text { (ppm) } & \text { (ppm) }\end{array}$

12AUG 85

04 SEP 84

$05 S E P 84$

06SEP 84

14 SEP84

17 SEP 84

19SEP84

20SEP84

21SEP84

24SEP84

25SEP84

26SEP 84

27 SEP 84

28SEP 84

$010 \mathrm{CT} 84$

020CT84

$030 C T 84$

$040 \mathrm{CT} 84$

050CT 84

090CT84

100CT 84

$110 \mathrm{CT} 84$

$120 \mathrm{CT} 84$

$150 \mathrm{CT} 84$

160CT 84

$170 \mathrm{CT} 84$

$180 \mathrm{CT} 84$

190CT 84

$220 \mathrm{CT} 84$

230 CT 34

240 TT 84

$250 \mathrm{CT} 84$

260 CT 84

290CT 84

$300 \mathrm{CT} 84$

310 CT 84

02NOV84

05NOV84

07NOV84

09NOV84

1 2NOV 84

14 NOV 84

16 NOV 84

19 NOV 84

21NOV84
0.02

0.03

0.04

0.03

0.01

0.01

0.01

0.01

0.01

0.01

0.01

0.01

0.01

0.01

0.01

0.01

0.01

0.01

0.02

0.21

57.00

112.50

130.00

99.00

31.00

10.60

5.70

2.80

1.43

0.62

0.27

0.19

0.14

0.11

0.02

0.20

0.40

0.34

0.06

0.08

0.05
127.00
2.30

0.040

0.238

0.020

0.020

0.020

0.020

0.020

0.020

0.020

0.020

0.061

0.092

0.020

0.020

0.020

0.020

0.020

0.191

37.590

75.630

77.810

13.810

52.110

19.795

5.942

3.028

1. 402

0.643

4. 112

0.092

0.073

0.020

0.020

0.076

0.152

0.380

0.235

0.020

0.03

0.03

0.03

0.03

0.03

0.03

0.03

0.09

2. 51

5. 21

6.69

5.60

3.69

2.49

1.73

0.97

0.62

0.51

0.39

0.26

0.21

0.18

0.15

0.14

0.12

0.10

0.09 
-TYPE=Caisson B $194 \mathrm{~cm}$ Depth Port

\begin{tabular}{|c|c|c|c|}
\hline $\begin{array}{l}\text { Sampling } \\
\text { Date }\end{array}$ & $\begin{array}{l}\text { Iodide } \\
\text { Concentration } \\
\text { (ppm) }\end{array}$ & $\begin{array}{l}\text { Bromide } \\
\text { Concentration } \\
\text { (ppm) }\end{array}$ & $\begin{array}{l}\text { Lithium } \\
\text { Concentration } \\
\text { (ppm) }\end{array}$ \\
\hline 23 NOV84 & 0.04 & 0.187 & 0.09 \\
\hline 26 NOV84 & 0.07 & 0.020 & . \\
\hline 28 NOV 84 & 0.03 & 0.317 & . \\
\hline 30 NOV84 & 0.04 & 0.020 & . \\
\hline 03DEC84 & 0.03 & 0.277 & . \\
\hline 05DEC84 & 0.02 & 0.125 & . \\
\hline 08DEC84 & 0.09 & 0.064 & . \\
\hline 09DEC84 & 0.02 & 0.616 & . \\
\hline 10DEC84 & 0.02 & 0.326 & . \\
\hline 11DEC84 & 0.03 & 0.580 & . \\
\hline 12DEC84 & 0.03 & 0.020 & . \\
\hline 13DEC84 & 0.05 & 0.020 & . \\
\hline 14DEC84 & 0.05 & 0.020 & . \\
\hline 15DEC84 & 0.10 & 0.077 & 0.06 \\
\hline 17DEC84 & 0.10 & 0.052 & 0.06 \\
\hline 18DEC84 & 0.04 & 0.020 & 0.06 \\
\hline 19DEC84 & 1.22 & 2.052 & 0.06 \\
\hline 20DEC84 & 25.80 & . & 0.05 \\
\hline 21DEC84 & 85.00 & . & 0.18 \\
\hline 22DEC84 & 128.00 & . & 0.12 \\
\hline 24DEC84 & 139.00 & . & 2.99 \\
\hline 26DEC84 & 112.00 & . & 6.20 \\
\hline 27DEC84 & 36.10 & . & 6.33 \\
\hline 28DEC84 & 12.60 & . & 5.30 \\
\hline 29DEC84 & 3.80 & . & 3.90 \\
\hline $31 D E C 84$ & 1. . 28 & . & 2.00 \\
\hline 02JAN85 & 0.46 & . & 0.80 \\
\hline 03JAN85 & 0.25 & . & 0.42 \\
\hline 04JAN85 & 0.19 & . & 0.30 \\
\hline 05JAN85 & 0.15 & . & 0.24 \\
\hline 07JAN85 & 0.14 & . & 0.19 \\
\hline 08JAN8 5 & 0.09 & . & 0.15 \\
\hline 09JAN85 & 0.09 & . & 0.13 \\
\hline 10JAN85 & 0.05 & . & . \\
\hline 11JAN85 & 0.05 & . & . \\
\hline 12JAN85 & 0.06 & . & . \\
\hline 14JAN85 & 0.08 & . & . \\
\hline 16JAN85 & 0.03 & . & . \\
\hline 18JAN85 & 0.02 & . & . \\
\hline 21JAN85 & 0.11 & . & . \\
\hline 23JAN85 & 0.04 & . & . \\
\hline 25JAN8 5 & 0.06 & . & . \\
\hline $28 \mathrm{JAN} 85$ & 0.09 & . & . \\
\hline 30JAN85 & 0.06 & $\cdot$ & . \\
\hline 01FEB85 & 0.05 & 0.049 & . \\
\hline
\end{tabular}




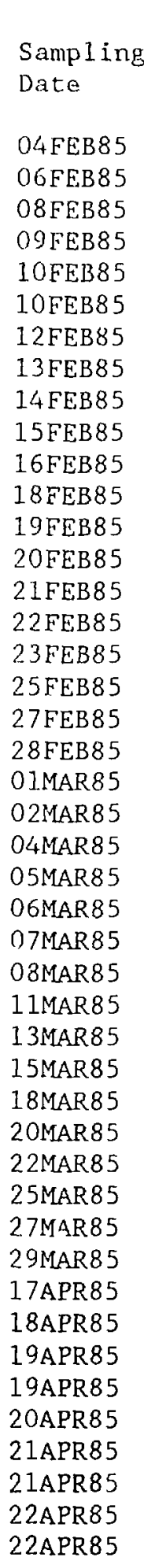

Iodide

Concentration (ppm)

1.5
124
120
54
17

0.10

0.02

0.02

0.04

0.01

0.03

0.05

0.01

0.18

0.01

1. 80

0.01

0.01

0.03

0.31

19.00

1.03 .00

1.51 .00

124.00

120.00

54.70

17.10

4.93

0.71

0.15

0.12

0.23

0.18

0.07

0.34

0.45

0.10

0.14

0.13

0.07

0.07

0.05

0.06

0.06

21.00

84.00

\section{Bromide \\ Concentration \\ (ppm)}

$$
0.020
$$

0.165

0.039

0.194

0.285

0.503

$$
\begin{gathered}
. \\
. \\
9.000
\end{gathered}
$$

58.000

85.000

80.000

71.000

33.000

8.000

2.000

0.04

0.04

0.05

2.79

6.04

6.05

5.79

5.33

3.24

1. 34

0.75

0.48

0.38

0.26
Concentration (ppm) 
Sampling

Date

23APR 85

23APR 85

24APR85

24APR8 5

25APR 85

25APR 85

26APR 85

26APR 85

28APR 85

28APR 85

29APR 85

29APR 85

$02 M A Y 85$

02 MAY 85

08 MAY85

15MAY85

18JUL85

19JUL85

20JUL85

22JUL85

23 JUL85

25JUL85

27JUL85

28 JUL85

29JUL85

30JUL85

01AUG85

02AUG 85

04AUG8 5

05AUG85

\section{Bromide Lithium \\ Concentration Concentration \\ (ppm) \\ ( $r: 3 \mathrm{~m})$}

$\begin{array}{cc}145.00 & 75.50 \\ 159.00 & 88.40 \\ 157.00 & 89.40 \\ 145.00 & 85.60 \\ 87.00 & . \dot{0} \\ 16.30 & 9.14 \\ . & . ं 52\end{array}$

4.20

1. 32

0.78

11.40

2.10

0.27

0.20

0.21

3.50
0.06

1.74

4.96

6.26

6.30

5.48

2.96

1.25

1.49

.

$\cdot$

0.16

0.13

0.28

4.38

6.89

6.45

4.36

2.10

0.84 
-TYPE=Caisson B $271 \mathrm{~cm}$ Depth Port

\begin{tabular}{|c|c|c|c|}
\hline $\begin{array}{l}\text { Sampling } \\
\text { Date }\end{array}$ & $\begin{array}{l}\text { Iodide } \\
\text { Concentration } \\
\text { (ppm) }\end{array}$ & $\begin{array}{l}\text { Bromide } \\
\text { Concentration } \\
\text { (ppm) }\end{array}$ & $\begin{array}{l}\text { Lithium } \\
\text { Concentration } \\
\text { (ppm) }\end{array}$ \\
\hline 17AUG84 & . & . & 0.020 \\
\hline 18AUG84 & . & . & 0.020 \\
\hline 19AUG84 & . & . & 0.020 \\
\hline 20AUG84 & . & . & 0.020 \\
\hline 21AUG84 & . & . & 0.020 \\
\hline 27AUG84 & . & 0.020 & . \\
\hline 31 AUG84 & . & . & 0.033 \\
\hline 06SEP84 & 0.03 & . & . \\
\hline 07SEP84 & . & 0.407 & . \\
\hline 11SEP84 & . & 0.020 & . \\
\hline 12SEP84 & . & 0.020 & . \\
\hline 13 SEP84 & $\cdot$ & 0.020 & . \\
\hline 17SEP84 & 0.04 & . & . \\
\hline 19SEP84 & 0.04 & . & . \\
\hline 20SEP84 & 0.04 & . & . \\
\hline 21SEP 84 & 0.04 & . & . \\
\hline 24SEP84 & 0.04 & . & . \\
\hline 25SEP84 & 0.04 & . & . \\
\hline 26SEP84 & 0.04 & . & . \\
\hline 27SEP84 & 0.04 & . & . \\
\hline 28SEP84 & 0.04 & . & . \\
\hline 010CT84 & 0.04 & . & . \\
\hline 020CT84 & 0.04 & . & . \\
\hline 030СT84 & 0.04 & . & . \\
\hline 040CT84 & 0.04 & . & . \\
\hline 050CT84 & 0.01 & . & . \\
\hline 090CT84 & 0.03 & 0.020 & . \\
\hline $100 \mathrm{CT} 84$ & 0.01 & 0.020 & . \\
\hline $110 \mathrm{CT} 84$ & 0.04 & 0.020 & . \\
\hline 120CT84 & 0.03 & 0.020 & . \\
\hline $150 \mathrm{CT} 84$ & 0.01 & 0.020 & 0.030 \\
\hline $160 \mathrm{CT} 84$ & 0.01 & 0.020 & 0.030 \\
\hline $170 \mathrm{CT} 84$ & 0.02 & 0.020 & 0.030 \\
\hline 180 CT84 & 0.03 & 0.020 & 0.030 \\
\hline $190 \mathrm{CT} 84$ & 0.16 & 1.070 & 0.030 \\
\hline $220 \mathrm{CT} 84$ & 22.00 & 7.590 & 0.030 \\
\hline $230 \mathrm{CT} 84$ & 34.00 & 22.515 & 0.030 \\
\hline $240 \mathrm{CT} 84$ & 38.00 & 23.495 & 0.030 \\
\hline $250 \mathrm{CT} 84$ & 30.20 & 20.106 & 0.030 \\
\hline $260 \mathrm{CT} 84$ & 18.80 & 11.607 & 0.050 \\
\hline $290 \mathrm{CT} 84$ & 6.60 & 4.169 & 0.830 \\
\hline $300 \mathrm{CT} 84$ & 7.90 & 4.783 & 1.130 \\
\hline $310 \mathrm{CT} 84$ & 10.80 & 6.475 & 1.070 \\
\hline 02NOV84 & 10.50 & 6.593 & 0.720 \\
\hline 05NOV84 & 7.60 & 4.542 & 0.750 \\
\hline
\end{tabular}




$\begin{array}{ll} & \text { Iodide } \\ \text { Sampling } & \text { Concentration } \\ \text { Date } & \text { (ppm) }\end{array}$

$\begin{array}{ll}\text { Bromide } & \text { Lithium } \\ \text { Concentration } & \text { Concentration } \\ \text { (ppm) } & \text { (ppm) }\end{array}$

07NOV84
09NOV84
12NOV84
14NOV84
16NOV84

12DEC 84

$13 \mathrm{DEC} 84$

14DEC84

$15 \mathrm{DEC} 84$

$17 \mathrm{DEC} 84$

$18 \mathrm{DEC} 84$

19 DEC 84

20DEC84

20DEC84

21DEC 84

21DEC 84

22DEC84

22DEC84

24DEC 84

24DEC84

26DEC84

26DEC 84

27DEC84

27DEC84

27DEC 84

28DEC84

28DEC 84

29DEC84

31 DEC84

31 DEC 84

02JAN8 5
5.90

1.04

3.26

4.90

6.10

1.09

0.46

0.24

0.16

0.11

0.10

0.06

0.06

0.04

0.04

0.04

0.42

0.05

0.04

0.09

3.419

2.994

2.042

4. 622

3.357

0.699

0.245

0.114

0.084

0.276

0.020

0.296

1. 625

0.490

0. 394

0.342

0.638

0.596

0.149

0.069

0.194

0.020

0.02

0.10

0.04

0.06

0.097

0.293

1.607

3. 15

31.00

90.00

131.00

130.00

10.80

83.00

33.20

16.760

52.390

75.610

67.140

23.225

17.612

5.144

1. 081

2.60
0.77

0.63

0.50

0.47

0.35

0.44

0.44

0.33

0.23

0.18

0.14

0.09

0.08

0.07

0.06

.

•

.

0.04

0.04

0.04

0.04

0.04

0.04

1.69

5.68

4.59

6.04

5.71

2. 11
0.397 


\begin{tabular}{|c|c|c|c|}
\hline $\begin{array}{l}\text { Sampling } \\
\text { Date }\end{array}$ & $\begin{array}{l}\text { Iodide } \\
\text { Concentration } \\
\text { (ppm) }\end{array}$ & $\begin{array}{l}\text { Bromide } \\
\text { Concentration } \\
\text { (ppm) }\end{array}$ & $\begin{array}{l}\text { Lithium } \\
\text { Concentration } \\
\text { (ppm) }\end{array}$ \\
\hline 02JAN85 & 1.05 & & 0.87 \\
\hline O3JAN85 & & 0.238 & . \\
\hline O3JAN 85 & 0.71 & . & 0.53 \\
\hline 04JAN8 5 & . & 0.215 & . \\
\hline 04JAN85 & 7.10 & . & 0.36 \\
\hline 05JAN85 & . & 0.148 & . \\
\hline 05JAN8 5 & 0.56 & . & 0.27 \\
\hline 07JAN85 & . & 0.136 & . \\
\hline 07JAN85 & 0.43 & . & 0.21 \\
\hline 08JAN85 & & 0.131 & . \\
\hline O8JAN85 & 0.38 & . & 0.17 \\
\hline O9JAN85 & & 0.129 & . \\
\hline 09JAN85 & 0.40 & . & 0.15 \\
\hline 10JAN85 & & 0.117 & . \\
\hline 10JAN85 & 0.31 & • & 0.13 \\
\hline 11JAN85 & & 0.127 & . \\
\hline 11JAN85 & 0.34 & & 0.12 \\
\hline 12JAN8 5 & . & 0.100 & . \\
\hline 12JAN85 & 0.25 & . & 0.10 \\
\hline 14JAN85 & . & 0.083 & . \\
\hline 14JAN8 5 & 0.27 & . & 0.09 \\
\hline 16JAN8 5 & 0.22 & . & 0.08 \\
\hline 18JAN85 & 0.12 & . & 0.07 \\
\hline 21JAN85 & . & 0.073 & . \\
\hline 21JAN85 & 0.05 & . & 0.06 \\
\hline 23JAN85 & 0.11 & . & 0.05 \\
\hline 25JAN85 & 0.18 & . & 0.05 \\
\hline 28JAN85 & . & 0.069 & \\
\hline 28JAN85 & 0.19 & . & 0.05 \\
\hline 30JAN85 & 0.16 & . & . \\
\hline 01FEB 85 & . & 0.082 & . \\
\hline 01FEB85 & 0.11 & & . \\
\hline O4FEB85 & . & 0.128 & . \\
\hline 04FEB85 & 0.11 & & . \\
\hline 06FEB8 5 & . & 0.545 & . \\
\hline 06FEB85 & 0.11 & . & . \\
\hline 08FEB8 5 & 0.10 & 0.060 & . \\
\hline 09FEB85 & 0.09 & . & . \\
\hline 10FEB85 & 0.08 & . & . \\
\hline $11 \mathrm{FEB} 85$ & 0.10 & . & . \\
\hline 12 FEB 85 & 0.09 & 0.020 & . \\
\hline $13 \mathrm{FEB} 85$ & 0.05 & . & . \\
\hline $14 \mathrm{FEB} 85$ & 0.06 & 0.071 & . \\
\hline 15FEB8 5 & 0.07 & . & . \\
\hline 16FEB85 & 0.06 & . & , \\
\hline
\end{tabular}




$\begin{array}{llll} & \text { Iodide } & \text { Bromide } & \text { Lithium } \\ \text { Sampling } & \text { Concentration } & \text { Concentration } & \text { Concentration } \\ \text { Date } & \text { (ppm) } & \text { (ppm) } & \text { (ppm) }\end{array}$

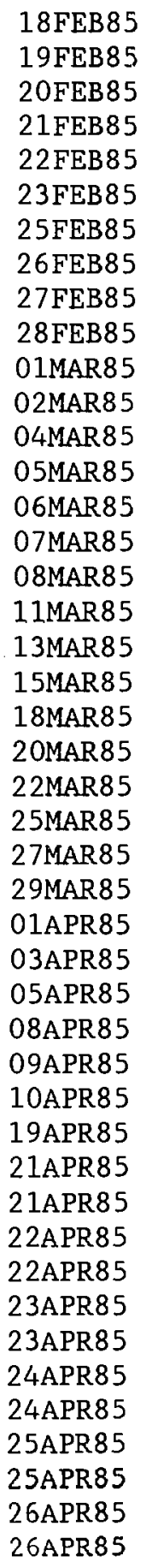

$$
\begin{array}{r}
0.07 \\
0.05 \\
0.05 \\
0.49 \\
11.50 \\
69.70 \\
147.00 \\
148.00 \\
120.00 \\
120.00 \\
76.00 \\
31.30 \\
7.36 \\
2.15 \\
1.26 \\
0.67 \\
0.32 \\
0.20 \\
0.49 \\
0.06 \\
0.23 \\
0.48 \\
0.37 \\
0.30 \\
0.29 \\
0.18 \\
0.17 \\
0.25 \\
0.41 \\
0.24 \\
0.22 \\
0.21 \\
151.00 \\
35.00 \\
90.00 \\
146.00 \\
. \\
152.00 \\
.
\end{array}
$$

\begin{tabular}{|c|c|}
\hline & \\
\hline 6.0 & 0.04 \\
\hline 40.0 & 0.04 \\
\hline 86.0 & 1.67 \\
\hline 81.0 & 5.75 \\
\hline 72.0 & 5.96 \\
\hline 49.0 & 5.72 \\
\hline 20.0 & 5.25 \\
\hline 4.0 & 3.34 \\
\hline . & 1.81 \\
\hline . & 0.98 \\
\hline . & 0.62 \\
\hline . & 0.42 \\
\hline . & 0.25 \\
\hline . & \\
\hline . & 0.12 \\
\hline . & 0.09 \\
\hline . & $\cdot$ \\
\hline . & . ' \\
\hline . & . \\
\hline . & . \\
\hline • & $\cdot$ \\
\hline . & $\cdot$ \\
\hline . & . \\
\hline . & . \\
\hline . & . \\
\hline . & • \\
\hline . & 0.04 \\
\hline & 0.04 \\
\hline 20.3 & . \\
\hline$\cdot$ & 0.04 \\
\hline 49.9 & $\therefore$ \\
\hline 75.7 & 0.04 \\
\hline & 0.97 \\
\hline 84.3 & \\
\hline & 3.07 \\
\hline 91.3 & · \\
\hline & 5.45 \\
\hline 85.7 & 6.39 \\
\hline
\end{tabular}

Lithium

(ppm) 


\begin{tabular}{|c|c|c|c|}
\hline $\begin{array}{l}\text { Sampling } \\
\text { Date }\end{array}$ & $\begin{array}{l}\text { Iodide } \\
\text { Concentration } \\
\text { (ppm) }\end{array}$ & $\begin{array}{l}\text { Bromide } \\
\text { Concentration } \\
\text { (ppm) }\end{array}$ & $\begin{array}{l}\text { Lithium } \\
\text { Concentration } \\
\text { (ppm) }\end{array}$ \\
\hline 28APR85 & . & 41.70 & . \\
\hline 28APR85 & 73.00 & . & 6.21 \\
\hline 29APR85 & . & 13.30 & . \\
\hline 29. IPR85 & 21.00 & . & 5.08 \\
\hline 02MAY85 & . & 1.40 & . \\
\hline 02MAY85 & 3.82 & . & 2.14 \\
\hline 08MAY85 & . & 0.27 & . \\
\hline 08MAY85 & 1.11 & . & 0.66 \\
\hline 15MAY85 & . & 0.19 & . \\
\hline 15MAY85 & 0.89 & . & 0.37 \\
\hline 14JUL85 & 4.50 & . & . \\
\hline 15JUL85 & 3.50 & . & . \\
\hline 16JUL85 & 3.30 & . & . \\
\hline $17 \mathrm{JUL} 85$ & 2.97 & . & . \\
\hline 18JUL85 & 1.50 & . & . \\
\hline 19JUL85 & 1.90 & . & . \\
\hline 20JUI 85 & . & . & 0.24 \\
\hline 20JUL85 & 0.82 & . & . \\
\hline 21JUL85 & . & . & 0.25 \\
\hline 21JUL85 & 0.60 & . & . \\
\hline 22JUL85 & . & . & 0.28 \\
\hline 22JUL85 & 1.90 & . & . \\
\hline 23JUL85 & . & . & 0.19 \\
\hline 23JUL85 & 0.66 & . & . \\
\hline 24JUL85 & . & . & 0.23 \\
\hline 24JUL85 & 2.02 & . & . \\
\hline 25JUL85 & . & . & 0.16 \\
\hline 25JUL85 & 0.48 & . & . \\
\hline 26JUL85 & . & . & 0.19 \\
\hline 26JUL85 & 0.92 & . & . \\
\hline 27JUL85 & 1.06 & . & 0.14 \\
\hline 28JUL85 & 24.40 & . & 0.13 \\
\hline 29JUL85 & 77.00 & . & 0.13 \\
\hline 30JUL85 & 130.00 & . & 0.13 \\
\hline 01 AUG85 & 149.00 & . & 2.85 \\
\hline $02 A U G 85$ & 119.00 & . & 5.36 \\
\hline 04AUG85 & 32.00 & . & 5.63 \\
\hline 05AUG85 & 7.80 & . & 3.84 \\
\hline 07AUG85 & 2.20 & . & 1.54 \\
\hline 09AUG85 & 1.18 & . & 0.68 \\
\hline 12AUG 85 & 0.82 & . & 0.30 \\
\hline 14AUG85 & . & . & 0.19 \\
\hline 16AUG85 & . & . & 0.15 \\
\hline 19AUG85 & 0.59 & . & . \\
\hline 21AUG 85 & 0.59 & . & . \\
\hline
\end{tabular}




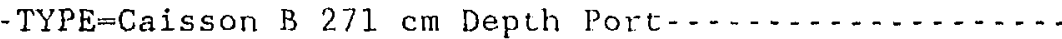

$\begin{array}{llll}\text { Sampling } & \begin{array}{l}\text { Iodide } \\ \text { Concentration } \\ \text { (ppm) }\end{array} & \begin{array}{l}\text { Bromide } \\ \text { Concentration } \\ \text { (ppin) }\end{array} & \begin{array}{l}\text { Lithium } \\ \text { Concentration } \\ \text { (ppm) }\end{array} \\ \text { 23AUg85 } & 0.6 & & \end{array}$


- TYPE $=$ Caisson B $347 \mathrm{~cm}$ Depth Port

\begin{tabular}{|c|c|c|c|}
\hline $\begin{array}{l}\text { Sampling } \\
\text { Date }\end{array}$ & $\begin{array}{l}\text { Iodide } \\
\text { Concentration } \\
\text { (ppm) }\end{array}$ & $\begin{array}{l}\text { Bromide } \\
\text { Concentration } \\
\text { (ppm) }\end{array}$ & $\begin{array}{l}\text { Lithium } \\
\text { Concentration } \\
\text { (ppm) }\end{array}$ \\
\hline 02JAN84 & 4.90 & . & . \\
\hline 03JAN84 & 29.00 & . & . \\
\hline 17 AUG84 & . & . & 0.02 \\
\hline $20 A U G 84$ & $\cdot$ & . & 0.02 \\
\hline O5SEP84 & 0.03 & . & . \\
\hline 06SEP84 & . & 0.187 & . \\
\hline 07SEP84́ & . & & 0.04 \\
\hline 11SEP 84 & . & 0.020 & . \\
\hline 17 SEP84 & 0.03 & . & . \\
\hline 21SEP84 & 0.03 & . & . \\
\hline 24 SEP 84 & 0.03 & . & . \\
\hline 26SEP 84 & 0.03 & . & . \\
\hline 28SEP84 & 0.03 & . & . \\
\hline 010CT84 & 0.03 & . & 0.04 \\
\hline 040CT84 & 0.03 & . & . \\
\hline $050 C T 84$ & 0.03 & . & 0.04 \\
\hline 090СТ84 & 0.03 & . & . \\
\hline $100 \mathrm{CT} 84$ & 0.03 & 0.020 & 0.04 \\
\hline $110 \mathrm{CT} 84$ & 0.03 & . & . \\
\hline $120 \mathrm{CT} 84$ & 0.02 & 0.020 & 0.04 \\
\hline $150 \mathrm{CT} 84$ & 0.02 & 0.020 & . \\
\hline $160 \mathrm{CT} 84$ & 0.02 & . & 0.04 \\
\hline $180 \mathrm{CT} 84$ & 0.02 & . & . \\
\hline $190 \mathrm{CT} 84$ & 0.01 & 0.020 & 0.04 \\
\hline $220 \mathrm{CT} 84$ & 0.15 & . & 0.04 \\
\hline $230 \mathrm{CT} 84$ & 0.15 & 0.020 & . \\
\hline $240 \mathrm{CT} 84$ & 0.15 & . & 0.04 \\
\hline $250 \mathrm{CT} 84$ & 0.15 & 0.020 & . \\
\hline $260 \mathrm{CT} 84$ & 0.12 & 0.020 & 0.04 \\
\hline $290 \mathrm{CT} 84$ & 0.50 & 7.518 & . \\
\hline $300 \mathrm{CT} 84$ & 36.00 & 24.300 & 0.04 \\
\hline $310 \mathrm{CT} 84$ & 78.00 & 51.010 & . \\
\hline 02NOV84 & 93.00 & 58.790 & 0.03 \\
\hline 05NOV84 & 71.00 & 41.000 & 0.15 \\
\hline 07NOV84 & 47.00 & 29.380 & 1.04 \\
\hline 09NOV84 & 12.10 & 10.615 & 3.95 \\
\hline 12 NOV 84 & 7.80 & 4.419 & 3.71 \\
\hline 14 NOV 84 & 2.95 & 1.361 & 2.18 \\
\hline 16 NOV84 & 1.09 & 0.146 & 1.31 \\
\hline 19 NOV 84 & 0.35 & 0.132 & 0.90 \\
\hline 21 NOV 84 & 0.22 & 0.010 & 0.76 \\
\hline 23 NOV 84 & 0.14 & 0.020 & 0.62 \\
\hline 26 NOV 84 & 0.12 & 0.163 & 0.40 \\
\hline 28NOV84 & 0.08 & 0.020 & 0.31 \\
\hline 30NOV84 & 0.08 & 0.440 & 0.25 \\
\hline
\end{tabular}




$\begin{array}{llll}\text { Sampling } & \begin{array}{l}\text { Iodide } \\ \text { Concentration }\end{array} & \begin{array}{l}\text { Bromide } \\ \text { Concentration }\end{array} & \begin{array}{l}\text { Lithium } \\ \text { Concentration } \\ \text { Date }\end{array} \\ \text { (ppm) } & \text { (ppm) } & \text { (ppm) }\end{array}$

O3DEC84

05DEC 84

$07 \mathrm{DEC} 84$

$08 D E C 84$

09DEC84

10DEC84

11DEC84

$12 D E C 84$

13DEC84

14DEC84

$15 D E C 84$

17 DEC 84

$18 \mathrm{DEC} 84$

$19 \mathrm{DEC} 84$

19 DEC 84

$20 D E C 84$

$20 \mathrm{DEC} 84$

21 DEC84

21DEC84

22DEC84

$22 \mathrm{DEC} 84$

24DEC84

24DEC8/4

26DEC 84

26DEC 84

27DEC 84

27 DEC 84

28 DEC 84

28DEC84

29DEC84

29DEC84

31 DEC 84

31 DEC84

02JAN85

03JAN8 5

04JAN85

04JAN85

05JAN85

05JAN85

07JAN 85

07JAN85

08JAN85

08JAN85

09J AN85

09J AN85
0.05

0.03

0.03

0.03

0.08

0.03

0.03

0.03

0.05

0.10

0.07

0.03

0.06

0.06

0.06

0.06

0.39

0.14

0.10

4.90

0.03

0.421

0.322

0.416

0.280

0.465

0.558

0.640

0.020

0.020

0.020

.

0.119

0.020

0.020

0.020

0.020

13.560

0.020

0.167

0.020

0.020

0.020

0. 020

3. 153

16.390

38.070

73.00

116.00

61.140

73.710

140.00

132.00

111.00
0.17

0.15

0.13 


$\begin{array}{ll}\text { Sampling } & \text { Iadide } \\ \text { Date } & \text { Concentration } \\ & \text { (ppm) }\end{array}$

$\begin{array}{ll}\text { Bromide } & \text { Lithium } \\ \text { Concentration } & \text { Concentration } \\ \text { (ppm) } & \text { (ppm) }\end{array}$

10JAN85

10JAN85

11 JAN85

11JAN 85

12JAN85

12JAN85

14JAN85

14JAN85

16JAN85

16JAN85

18JAN85

$18 \mathrm{JAN} 85$

21JAN85

21JAN85

23JAN85

23JAN85

25JAN85

28JAN85

30JAN 85

01FEB 85

OIFEB8 5

04FEB85

04FEB85

06FEB85

06FEB 85

08FEB85

O9FEB 85

10FEB 85

11FEB 85

12 FEB8 5

13FEB85

$14 \mathrm{FEB} 85$

15 FEB85 5

$16 \mathrm{FEB} 85$

$18 F E B 85$

19FEB 85

20FEB8 5

21FEB 5

22 FEB 85

23FEB 85

25FEE85

26FEB 85

27FEB 85

28FEB85

O1MAR 85

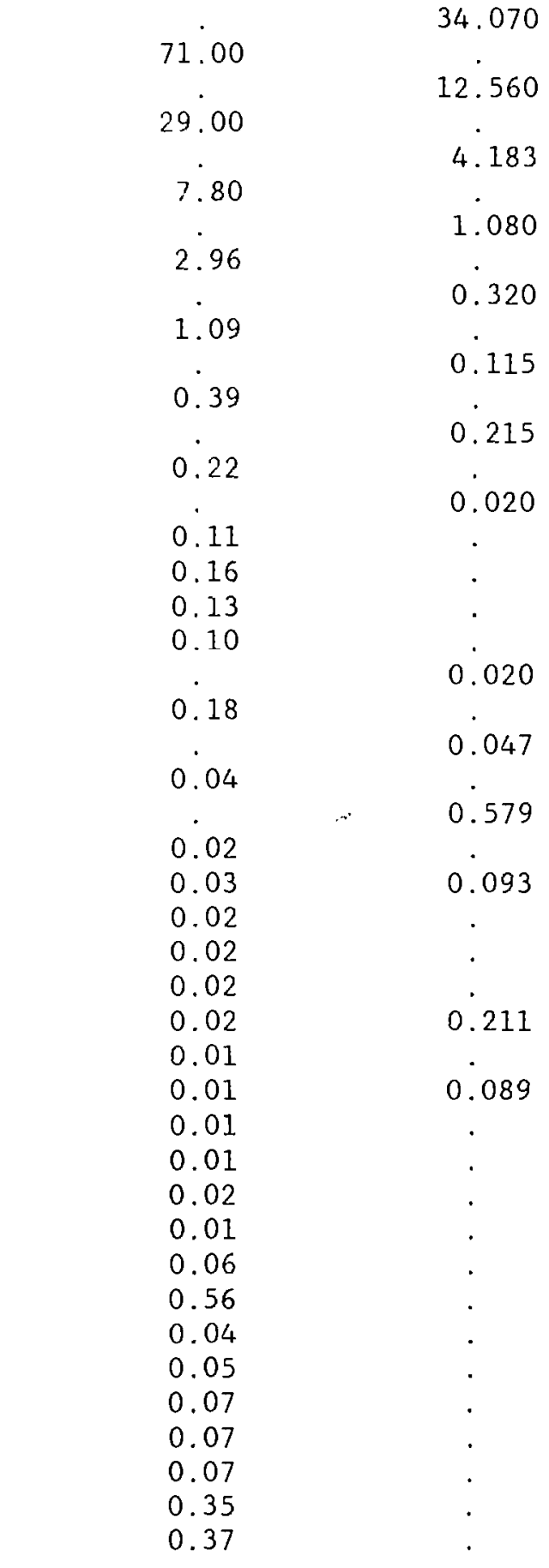

34.070

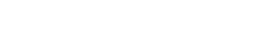


- TYPE=Caisson B $347 \mathrm{~cm}$ Depth Port

$\begin{array}{llll} & \text { Iodide } & \text { Bromide } & \text { Lithium } \\ \text { Sampling } & \text { Concentration } & \text { Concentration } & \text { Concentration } \\ \text { Date } & \text { (ppm) } & \text { (ppm) } & \text { (ppm) }\end{array}$

\begin{tabular}{|c|c|}
\hline 02MAR8 5 & 0.28 \\
\hline 04MAR85 & 0.40 \\
\hline 05MAR8 5 & 0.36 \\
\hline 06MAR85 & 2.74 \\
\hline 07MAR85 & 18.20 \\
\hline O8MAR85 & 60.00 \\
\hline 11MAR85 & 118.00 \\
\hline 13MAR85 & 100.00 \\
\hline 15MAR85 & 49.00 \\
\hline 18MAR85 & 12.40 \\
\hline 20MAR8 5 & 5.50 \\
\hline 22MAR85 & 4.04 \\
\hline 25 MAR 85 & 2.88 \\
\hline 27MAR85 & 2.08 \\
\hline 29MAR 85 & 1.90 \\
\hline 01APR85 & 0.39 \\
\hline 03APR85 & 1.50 \\
\hline 05APR85 & 1.30 \\
\hline 08APR85 & 1.21 \\
\hline 09APR85 & 0.86 \\
\hline 10APR85 & • \\
\hline 15APR85 & . \\
\hline 21APR8 5 & . \\
\hline 21APR 85 & 0.55 \\
\hline 22APR85 & . \\
\hline 22APR85 & 0.49 \\
\hline $23 \mathrm{APR} 85$ & . \\
\hline 23APR 85 & 0.52 \\
\hline 24APR 85 & 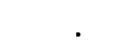 \\
\hline 24APR85 & 0.50 \\
\hline 25APR85 & 0.78 \\
\hline 26APR 85 & • \\
\hline 26APR85 & 0.39 \\
\hline 28APR85 & \\
\hline 28APR85 & 0.52 \\
\hline 29APR85 & \\
\hline 29APR85 & 0.37 \\
\hline O2MAY85 & \\
\hline O2MAY85 & 0.71 \\
\hline O8MAY85 & . \\
\hline 08MAY85 & 86.00 \\
\hline 15MAY85 & 213.00 \\
\hline 21JUL85 & . \\
\hline 23JUL85 & \\
\hline 23JUL85 & 80.00 \\
\hline
\end{tabular}

\begin{tabular}{|c|c|}
\hline 2.00 & 0.05 \\
\hline 11.00 & 0.05 \\
\hline 38.00 & 0.06 \\
\hline 75.00 & 0.16 \\
\hline 65.00 & \\
\hline 27.00 & 4.33 \\
\hline 2.00 & 4.43 \\
\hline . & 2.85 \\
\hline . & 1.62 \\
\hline . & 0.90 \\
\hline . & 0.58 \\
\hline . & 0.37 \\
\hline · & $\circ$ \\
\hline . & 0.22 \\
\hline$\cdot$ & $\cdot$ \\
\hline . & i. \\
\hline . & 0.12 \\
\hline & 0.10 \\
\hline 0.19 & . \\
\hline & 0.07 \\
\hline 0.15 & 0.07 \\
\hline 0.08 & . \\
\hline . & 0.07 \\
\hline 0.44 & · \\
\hline$\cdot$ & 0.07 \\
\hline$\cdot$ & 0.09 \\
\hline 0.48 & $\cdot$ \\
\hline$\cdot$ & 0.07 \\
\hline 0.09 & $\cdot$ \\
\hline 013 & 0.07 \\
\hline 0.13 & '. \\
\hline 0.14 & 0.08 \\
\hline & C.07 \\
\hline 6.40 & $\cdot$ \\
\hline · & 0.08 \\
\hline$\cdot$ & 0.09 \\
\hline . & 2.84 \\
\hline . & 13.42 \\
\hline
\end{tabular}




$\begin{array}{llll} & \text { Iodide } & \text { Bromide } & \text { Lithium } \\ \text { Sampling } & \text { Concentration } & \text { Concentration } & \text { Concentration } \\ \text { Date } & (\text { ppm) } & \text { (ppm) } & \text { (ppm) }\end{array}$

24JUL85

24JUL85

26JUL85

26 JUL85

$27 \mathrm{JUL} 85$

28 JUL8 5

29JUL8 5

30 JUL 85

01AUG 85

02AUG 85

04AUG85

06AUG85

08AUG85

12 AUG8 5

15 AUG8 5

$19 A U G 85$

23 AUG 85

$$
\begin{array}{r}
23.00 \\
10.60 \\
9.20 \\
6.10 \\
4.00 \\
3.20 \\
1.89 \\
3.76 \\
2.10 \\
27.00 \\
250.00 \\
148.00 \\
11.00 \\
4.90
\end{array}
$$

10.21

9.43

7.72

6.01

6.70

4. 37

3. 29

2. 19

1.57

1.83 


$\begin{array}{llll} & \text { Iodide } & \text { Bromide } & \text { Lithium } \\ \text { Sampling } & \text { Concentration } & \text { Concentration } & \text { Concentration } \\ \text { Date } & \text { (ppm) } & \text { (ppm) } & \text { (ppm) }\end{array}$

17 AUG84 20AUG84 21AUG 84 05SEP84 06SEP84 $07 S E P 84$ 11SEP84 17SEP84 21SEP84 24SEP84 26SEP 84 28 SEP 84 $010 \mathrm{CT} 84$ $030 \mathrm{CT} 84$ 050CT 84 $090 \mathrm{CT} 84$ $100 \mathrm{CT} 84$ 110CT 84 $150 \mathrm{CT} 84$ $160 \mathrm{CT} 84$ $170 \mathrm{CT} 84$ $180 \mathrm{CT} 84$ $220 \mathrm{CT} 84$ $230 \mathrm{CT} 84$ $240 \mathrm{CT} 84$ $250 \mathrm{CT} 84$ $260 \mathrm{CT} 84$ $290 \mathrm{CT} 84$ 300CT 84 $310 \mathrm{CT} 84$ 02NOV 84 05 NOV 84 07NOV84 09NOV84 12 NOV 84 14 NOV 84 16 NOV 84 19 NOV 84 21NOV84 23 NOV 84 26NOV84 28NOV84 30 NOV 84 O3DEC84 O5DEC84

.

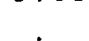

0.03

0.03

0.03

0.02

0.03

0.03

0.03

0.03

0.03

0.03

0.03

0.03

0.02

0.02

0.02

0.15

0.15

0.15

0.15

0.01

0.10

0.10

0.10

16.10

60.00

60.00

14.40

1.88

0.49

0.20

0.11

0.07

0.09

0.06

0.06

0.03

0.02

\section{Bromide \\ (ppm)}

\begin{tabular}{|c|c|}
\hline . & 0.02 \\
\hline . & 0.02 \\
\hline & 0.02 \\
\hline 0.020 & · \\
\hline 0.547 & $\cdot$ \\
\hline & 0.04 \\
\hline 0.020 & · \\
\hline$\cdot$ & $\cdot$ \\
\hline$\cdot$ & $\cdot$ \\
\hline$\cdot$ & $\cdot$ \\
\hline$\cdot$ & · \\
\hline · & · \\
\hline . & · \\
\hline$\cdot$ & $\cdot$ \\
\hline$\cdot$ & $\cdot$ \\
\hline 0.020 & 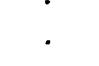 \\
\hline 0.020 & . \\
\hline 0.020 & \\
\hline$\cdot$ & . \\
\hline 0.020 & . \\
\hline . & . \\
\hline 0.020 & 0.04 \\
\hline 0.020 & 0.04 \\
\hline 0.020 & 0.04 \\
\hline 0.020 & 0.04 \\
\hline 0.020 & 0.04 \\
\hline 0.120 & 0.04 \\
\hline 9.800 & 0.04 \\
\hline 54.990 & 0.04 \\
\hline 39.790 & 0.04 \\
\hline 7.835 & 0.38 \\
\hline 1.020 & 2.33 \\
\hline 0.362 & 2.32 \\
\hline 0.078 & 1.22 \\
\hline 0.010 & 0.80 \\
\hline 0.020 & 0.60 \\
\hline 0.020 & 0.43 \\
\hline 0.365 & 0.37 \\
\hline 0.257 & 0.32 \\
\hline 0.131 & \\
\hline 0.277 & 0.21 \\
\hline
\end{tabular}




\begin{tabular}{|c|c|c|c|}
\hline $\begin{array}{l}\text { Sampling } \\
\text { Date }\end{array}$ & $\begin{array}{l}\text { Iodide } \\
\text { Concentration } \\
\text { (ppm) }\end{array}$ & $\begin{array}{l}\text { Bromide } \\
\text { Concentration } \\
\text { (ppm) }\end{array}$ & $\begin{array}{l}\text { Jithium } \\
\text { Concentration } \\
\text { (ppm) }\end{array}$ \\
\hline $07 \mathrm{DEC} 84$ & 0.02 & 0.405 & 0.19 \\
\hline 08DEC84 & 0.02 & 0.186 & . \\
\hline 09DEC84 & 0.04 & 0.179 & 0.17 \\
\hline $10 \mathrm{DEC} 84$ & 0.02 & 0.594 & . \\
\hline 11 DEC 84 & 0.02 & 0.343 & . \\
\hline $12 \mathrm{DEC} 84$ & 0.02 & 0.020 & . \\
\hline $13 \mathrm{DEC} 84$ & . & 0.020 & . \\
\hline 14DEC84 & · & 0.020 & . \\
\hline $15 \mathrm{DEC} 84$ & 0.01 & & . \\
\hline 17DEC 84 & 0.10 & 0.089 & . \\
\hline 18DEC 84 & 0.03 & & . \\
\hline $19 D E C 84$ & 0.02 & 0.020 & . \\
\hline 20DEC84 & $\cdot$ & 0.020 & . \\
\hline 20DEC 84 & 0.03 & · & . \\
\hline 21DEC84 & . & 0.020 & . \\
\hline $21 \mathrm{DEC} 84$ & 0.03 & & . \\
\hline $22 \mathrm{DEC} 84$ & & 0.020 & . \\
\hline $22 \mathrm{DEC} 84$ & 0.02 & • & . \\
\hline 24DEC84 & . & 0.020 & . \\
\hline $24 D E C 84$ & 0.03 & • & . \\
\hline 26DEC84 & . & 0.133 & . \\
\hline 26DEC84 & 0.25 & · & . \\
\hline $27 D E C 84$ & , & 0.103 & . \\
\hline $27 \mathrm{DEC} 84$ & 0.19 & & . \\
\hline $28 D E C 84$ & · & 0.519 & . \\
\hline $28 D E C 84$ & 0.06 & & . \\
\hline 29DEC 84 & . & 0.040 & . \\
\hline 29DEC 84 & 0.03 & & . \\
\hline $31 \mathrm{DEC} 84$ & $\cdot$ & 0.020 & . \\
\hline $31 \mathrm{DEC} 84$ & 0.02 & . & . \\
\hline 02JAN8 5 & . & 0.020 & . \\
\hline 02JAN85 & 0.03 & . & . \\
\hline 03JAN85 & $\cdot$ & 0.020 & . \\
\hline 03JAN85 & 0.04 & . & . \\
\hline 04JAN85 & $\cdot$ & 0.020 & . \\
\hline 04JAN85 & 0.07 & $\cdot$ & . \\
\hline 05JAN85 & . & 0.054 & . \\
\hline 05JAN85 & 0.10 & . & . \\
\hline 07JAN85 & . & 0.433 & . \\
\hline 07JAN8 5 & 0.68 & & . \\
\hline O8JAN85 & & 3.228 & . \\
\hline 08JAN85 & 5.40 & & . \\
\hline 09JAN85 & . & 12.270 & . \\
\hline 09JAN8 5 & 22.00 & 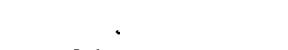 & . \\
\hline 10JAN85 & . & 29.650 & . \\
\hline
\end{tabular}




$\begin{array}{llll}\text { Sampling } & \begin{array}{l}\text { Iodide } \\ \text { Concentration }\end{array} & \begin{array}{l}\text { Bromide } \\ \text { Concentration }\end{array} & \begin{array}{l}\text { Lithium } \\ \text { Concentration } \\ \text { (ppm) }\end{array} \\ \text { (ppm) } & \text { (ppm) }\end{array}$

11JAN8 5

12JAN85

12JAN85

14JAN85

14JAN85

16JAN85

16JAN85

18JAN85

18JAN85

21JAN85

21JAN85

23JAN85

23JAN85

25JAN85

25JAN85

28JAN85

28JAN85

30JAN85

30 JAN8 5

01FEB 85

01FEB 85

04FEB8 5

04FEB8 5

06FEB 85

06FEB85

08FEB85

09FEB85

10FEB 85

11 FEB 85

12FEB85

13FEB 85

14FEB 85

$15 F E B 85$

$16 \mathrm{FEB} 85$

$18 \mathrm{FEB} 85$

19 FEB 85

20FEB8 5

21 FEB 85

22 FEB 85

$23 \mathrm{FEB} 85$

25FEB 85

26 FEB 85

27FEB 85

28FEB8 5

01MAR8 5

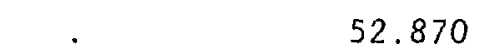

129.00

143.00

93.00

16.80

1.88

0.55

0.38

0.26

0.18

0.13

0.08

0.05

0.04

0.03

0.03

0.04

0.03

0.01

0.01

0.02

0.04

0.03

0,02

0.02

0.54

0.08

0.05

0.15

0.05

0.05

0.38

0.38
52.870

69.560

72.440

45.750

11.620

1.173

0.175

0.416

0.104

0.113

0.051

0.070

0.020

0.068

0.174
0.04

0.04

0.46

3.42

4.45

3. 16

1.83

0.89

0.60

0.45 


\begin{tabular}{|c|c|c|c|}
\hline $\begin{array}{l}\text { Sampling } \\
\text { Date }\end{array}$ & $\begin{array}{l}\text { Iodide } \\
\text { Concentration } \\
\text { (ppm) }\end{array}$ & $\begin{array}{l}\text { Bromide } \\
\text { Concentration } \\
\text { (ppm) }\end{array}$ & $\begin{array}{l}\text { Lithium } \\
\text { Concentration } \\
\text { (ppm) }\end{array}$ \\
\hline 02MAR8 5 & 0.28 & & . \\
\hline 04 MAR 85 & 0.39 & . & . \\
\hline O5MAR8 5 & 0.39 & . & . \\
\hline 06MAR8 5 & 0.39 & . & . \\
\hline 07MAR85 & 0.39 & . & . \\
\hline 08MAR85 & 0.39 & . & . \\
\hline 11MAR85 & 0.14 & . & . \\
\hline 13MAR 85 & 15.80 & 11.000 & . \\
\hline 15MAR85 & 98.00 & 56.000 & . \\
\hline 18MAR 85 & 131.00 & 80.000 & 0.06 \\
\hline 20MAR85 & . & 60.700 & • \\
\hline 2OMAR85 & 102.00 & . & 1.58 \\
\hline 22MAR 85 & & 22.500 & , \\
\hline 22MAR 85 & 42.30 & . & 3.96 \\
\hline 25 MAR 85 & & 3.380 & . \\
\hline $25 \mathrm{MAR} 85$ & 7.26 & . & 4.30 \\
\hline 27MAR 85 & . & 0.060 & . \\
\hline 27MAR8 5 & 1.59 & . & 3.24 \\
\hline 29MAR85 & & 0.650 & . \\
\hline 29MAR8 & 0.70 & • & 1.99 \\
\hline 01APR85 & 1.70 & . & 0.27 \\
\hline 03APR8 5 & 0.35 & . & 0.68 \\
\hline 05APR 85 & 0.30 & . & 0.47 \\
\hline 08APR8 5 & 0.21 & . & 0.35 \\
\hline 09APR8 5 & 0.14 & . & . \\
\hline 10APR8 5 & . & . & 0.25 \\
\hline 15APR85 & . & . & 0.15 \\
\hline 21APR 85 & . & . & 0.11 \\
\hline 22APR 85 & . & 0.004 & . \\
\hline 22APR 85 & 0.07 & . & . \\
\hline 23APR 85 & . & 0.080 & . \\
\hline 23APR85 & 0.06 & & . \\
\hline 24APR 85 & . & 0.180 & . \\
\hline 24APR85 & 0.16 & . & . \\
\hline 25APR85 & . & 0.090 & . \\
\hline 25APR 85 & 0.08 & • & . \\
\hline 26APR85 & . & 0.400 & . \\
\hline 26APR85 & 0.05 & . & 0.08 \\
\hline 28APR85 & & 0.180 & $\cdot$ \\
\hline 28APR85 & 0.07 & & 0.08 \\
\hline 29APR85 & . & 0.270 & . \\
\hline 29APR85 & 0.07 & . & 0.08 \\
\hline 02MÂY85 & $\cdot$ & 0.280 & . \\
\hline 02MAY85 & 0.05 & & 0.07 \\
\hline 08MAY 85 & . & 0.150 & . \\
\hline
\end{tabular}




$\begin{array}{llll}\text { Sampling } & \begin{array}{l}\text { Iodide } \\ \text { Concentration }\end{array} & \begin{array}{l}\text { Bromide } \\ \text { Concentration } \\ \text { (ppm) }\end{array} & \begin{array}{l}\text { Lithium } \\ \text { Concentration } \\ \text { Date }\end{array} \\ \text { (ppm) } & \text { (ppm) }\end{array}$

\begin{tabular}{|c|c|c|c|}
\hline 08MAY85 & 0.31 & & 0.07 \\
\hline 15MAY85 & . & 17.2 & \\
\hline 15MAY8: & 58.00 & . & 0.08 \\
\hline 18JUL85 & & . & 2.94 \\
\hline 18JUL85 & 280.00 & . & • \\
\hline 22JUL85 & $\cdot$ & . & 3.13 \\
\hline 22JUL85 & 72.00 & . & . \\
\hline 23JUL85 & & . & 3.30 \\
\hline 23JUL85 & 96.00 & . & . \\
\hline 24JUL85 & $\cdot$ & . & 3.86 \\
\hline 24JUL85 & 67.00 & . & . \\
\hline 25JUL85 & $\cdot$ & . & 4.39 \\
\hline 25JUL85 & 39.60 & . & . \\
\hline 26JUL85 & . & . & 5.62 \\
\hline 26JUL85 & 28.20 & . & \\
\hline 27JUL85 & 20.70 & . & 5.92 \\
\hline 28JUL.85 & 12.30 & . & 4.59 \\
\hline 29JUL85 & 12.10 & . & 5.77 \\
\hline 30JUL85 & 7.10 & . & 5.51 \\
\hline 01AUG85 & 4.20 & . & 4.33 \\
\hline 02AUG85 & 1.83 & . & 3.61 \\
\hline 04AUG85 & 1.53 & . & 2.68 \\
\hline 05AUG85 & 1.51 & . & 2.21 \\
\hline 07AUG85 & 1.96 & . & 1.88 \\
\hline 09AUG85 & 1.48 & . & 1.70 \\
\hline 12AUG 85 & 1.24 & . & 1.36 \\
\hline 14AUG85 & 2.70 & . & 1.03 \\
\hline 16AUG85 & 45.00 & . & 0.79 \\
\hline 19AUG85 & 132.00 & . & 0.60 \\
\hline 21AUG85 & 149.00 & . & 1.14 \\
\hline 23AUG85 & 74.00 & . & 3.99 \\
\hline 28AUG85 & 4.40 & . & 4.35 \\
\hline
\end{tabular}


TYPE $=$ Caisson B Vertical Sampler at 43

\begin{tabular}{|c|c|c|c|}
\hline $\begin{array}{l}\text { Sampling } \\
\text { Date }\end{array}$ & $\begin{array}{c}\text { Indide } \\
\text { concentration } \\
\text { (ppm) }\end{array}$ & $\begin{array}{c}\text { Bromide } \\
\text { concentration } \\
(\mathrm{ppm})\end{array}$ & $\begin{array}{c}\text { Lithium } \\
\text { concentration } \\
\text { (ppm) }\end{array}$ \\
\hline $15 \mathrm{FEB} 85$ & 142.00 & 72.98 & 6.61 \\
\hline $16 \mathrm{FEB} 85$ & 115.00 & 62.33 & 6.23 \\
\hline $18 \mathrm{FEB} 85$ & 97.20 & 6.16 & 1.32 \\
\hline 19 FEB 85 & 0.90 & $\cdot$ & 0.45 \\
\hline 20FEB85 & 0.51 & . & 0.13 \\
\hline 21 FEB 85 & 0.51 & . & 0.07 \\
\hline 23 FEB 85 & 0.05 & . & 0.04 \\
\hline $25 \mathrm{FEB} 85$ & 0.21 & . & 0.05 \\
\hline 26 FEB 85 & 0.05 & . & 0.04 \\
\hline 2.7FEB 85 & 0.04 & . & $\cdot$ \\
\hline $28 F E B 85$ & 0.04 & . & . \\
\hline 05MAR85 & 0.06 & . & . \\
\hline 06MAR85 & $0 . \therefore 0$ & . & . \\
\hline 07MAR85 & 0.39 & . & . \\
\hline 08MAR85 & 0.41 & . & . \\
\hline 11) LAR 85 & 0.40 & . & . \\
\hline 13MAR8 5 & 0.70 & . & . \\
\hline 15MAR 85 & 0.40 & . & . \\
\hline 18MAR85 & 0.16 & . & . \\
\hline 20MAR8 5 & 0.65 & . & . \\
\hline 22MAR85 & 0.20 & . & . \\
\hline 25MAR85 & 0.07 & . & . \\
\hline 27MAR 85 & 2.17 & . & . \\
\hline 29MAR85 & 0.02 & . & \\
\hline 10APR85 & 13.10 & . & 0.07 \\
\hline 11APR85 & 0.89 & . & 0.04 \\
\hline 12APR85 & 151.00 & . & 6.04 \\
\hline 13APR8 5 & 157.00 & . & 7.20 \\
\hline 14APR8 5 & 178.00 & . & 7.63 \\
\hline 15APR 85 & 174.00 & . & 7.61 \\
\hline 16APR85 & 100.00 & . & 6.23 \\
\hline 17APR85 & 43.00 & . & 2.93 \\
\hline 18APR8 5 & 14.30 & . & 1.33 \\
\hline 19APR85 & 4.10 & . & 0.55 \\
\hline 21APR85 & 0.19 & . & 0.07 \\
\hline 21APR 85 & 0.36 & . & 0.12 \\
\hline 23APR85 & 0.11 & . & 0.05 \\
\hline 24APR85 & 0.09 & . & $\cdot$ \\
\hline 25APR85 & 0.11 & . & . \\
\hline 26APR85 & 0.08 & . & . \\
\hline 28APR85 & 0.06 & . & . \\
\hline 29APR 85 & 0.07 & . & . \\
\hline 02MAY85 & 0.07 & . & . \\
\hline 13JUL85 & 213.90 & . & 5.87 \\
\hline 14JUL85 & 192.00 & . & 7.85 \\
\hline
\end{tabular}


- TYPE $=$ Caisson B Vertical Sampler at 43

$\mathrm{cm} \ldots \ldots \ldots \ldots$

Iodide Bromide Lithium

Sampling Date

15JUL85

16JUL85

18JUL8 5

20JUL85

21JUL85

22JUL85

23.JUL 85

24JUL85 concentration (ppn)

200.00

155.00

46.10

2.90

2.90

0.50

0.37

0.29 concentration

(ppm)

8.49

7. 37

0.44

0.35 
- TYPE=Caisson B Vertica1 Sampler at 58

\begin{tabular}{|c|c|c|c|}
\hline $\begin{array}{l}\text { Sampling } \\
\text { Date }\end{array}$ & $\begin{array}{c}\text { Iodide } \\
\text { concentration } \\
\text { (ppm) }\end{array}$ & $\begin{array}{c}\text { Bromide } \\
\text { concentration } \\
\text { (ppm) }\end{array}$ & $\begin{array}{c}\text { Lithium } \\
\text { concentration } \\
\text { (ppm) }\end{array}$ \\
\hline $15 \mathrm{FEB} 85$ & 177.00 & 84.08 & 7.03 \\
\hline 16 FEB 85 & 152.00 & 78.00 & 7.11 \\
\hline $18 \mathrm{FEB} 85$ & 112.00 & 69.00 & 6.35 \\
\hline 19FEB85 & 57.00 & . & 4.90 \\
\hline 20FEB85 & 18.40 & 9.00 & 2.71 \\
\hline 21 FEB 85 & 2.43 & 0.40 & 1.10 \\
\hline 22 FEB 85 & 0.05 & . & 0.38 \\
\hline 23FEB85 & 0.18 & . & 0.15 \\
\hline 25 FEB 85 & 0.15 & . & 0.07 \\
\hline 26 FEB 85 & 0.09 & . & 0.06 \\
\hline 28 FEB 85 & 0.36 & . & . \\
\hline 01MAR85 & 0.36 & . & . \\
\hline 02MAR 85 & 0.39 & . & . \\
\hline 04MAR85 & 0.29 & . & . \\
\hline 05MAR85 & 0.22 & . & . \\
\hline 06MAR85 & 0.34 & . & . \\
\hline 07MAR85 & 0.39 & . & . \\
\hline 08MAR85 & 0.39 & . & . \\
\hline 11MAR85 & 0.39 & . & . \\
\hline 13MAR85 & 0.15 & . & . \\
\hline 15MAR 85 & 0.43 & . & . \\
\hline 18MAR85 & 0.56 & . & . \\
\hline 20MAR85 & 0.12 & . & . \\
\hline 22MAR85 & 0.03 & . & . \\
\hline 25MAR85 & 0.03 & . & . \\
\hline 27MAR85 & 0.05 & . & . \\
\hline 27MAR85 & 0.08 & . & 0.05 \\
\hline 29MAR85 & 0.01 & . & . \\
\hline 01APR85 & & . & 2.02 \\
\hline 10APR 85 & 0.14 & . & 0.04 \\
\hline 11APR85 & 0.09 & . & 0.04 \\
\hline 12APR85 & 68.00 & . & 1.03 \\
\hline 12.APR 85 & 138.00 & . & 4.97 \\
\hline 14APR85 & 175.00 & . & 7.13 \\
\hline 15APR 85 & 178.00 & . & 7.24 \\
\hline 16APR 85 & 164.00 & . & 7.17 \\
\hline 1.7APR85 & 150.00 & . & 7.05 \\
\hline 18APR 85 & 58.00 & . & 4.63 \\
\hline 19APR85 & 10.60 & . & 1.44 \\
\hline 21APR8 5 & 1.66 & . & 0.17 \\
\hline 22APR 85 & 0.56 & . & 0.10 \\
\hline 23APR85 & 0.34 & . & 0.07 \\
\hline 24APR85 & 0.19 & . & 0.06 \\
\hline 25AFR85 & 0.13 & . & 0.05 \\
\hline 26APR85 & 0.09 & . & 0.04 \\
\hline
\end{tabular}


TYPE=Caisson B Vertical Sampler at 58

$\begin{array}{lccc}\begin{array}{c}\text { Sampling } \\ \text { Date }\end{array} & \begin{array}{c}\text { Iodide } \\ \text { concentration } \\ \text { (ppm) }\end{array} & \begin{array}{c}\text { Bromide } \\ \text { concentration } \\ \text { (ppm) }\end{array} & \begin{array}{c}\text { Lithium } \\ \text { concentration } \\ \text { (ppm) }\end{array} \\ \text { 28APR85 } & 0.08 & . & 0.04 \\ \text { 29APR85 } & 0.08 & . & . \\ \text { 02MAY85 } & 0.07 & . & . \\ \text { 08MAY85 } & 0.10 & . & . \\ \text { 13JUL85 } & 0.17 & . & 0.13 \\ \text { 14JUL85 } & 45.00 & . & 0.08 \\ \text { 15JUL85 } & 158.00 & . & 5.70 \\ \text { 16JUL85 } & 169.00 & . & 7.41 \\ \text { 17JUL85 } & 166.00 & . & 7.49 \\ \text { 18JUL85 } & 151.00 & . & 6.71 \\ \text { 19JUL85 } & 128.00 & . & 2.70 \\ \text { 20JUL85 } & 26.00 & . & 0.63 \\ \text { 21JUL85 } & 2.60 & . & .\end{array}$


- TYPE $=$ Caisson B Vertical Sampler at 73

\begin{tabular}{|c|c|c|c|}
\hline $\begin{array}{l}\text { Sampling } \\
\text { Date }\end{array}$ & $\begin{array}{c}\text { Iodide } \\
\text { concentration } \\
(\mathrm{ppm})\end{array}$ & $\begin{array}{c}\text { Bromide } \\
\text { concentration } \\
(\mathrm{ppm})\end{array}$ & $\begin{array}{c}\text { Lithium } \\
\text { concentration } \\
(\mathrm{ppm})\end{array}$ \\
\hline $15 \mathrm{FEB} 85$ & 132.00 & 59.54 & 2.12 \\
\hline 16FEB8 5 & 155.00 & 81.49 & 3.51 \\
\hline $18 \mathrm{FEB} 85$ & 170.00 & 86.00 & 6.29 \\
\hline 19FEB85 & 155.00 & . & 6.67 \\
\hline $20 \mathrm{FEB} 85$ & 136.00 & 76.00 & 6.46 \\
\hline $21 \mathrm{FEB} 85$ & 100.00 & 47.00 & 5.89 \\
\hline $22 \mathrm{FEB} 85$ & 89.20 & 15.00 & 4.33 \\
\hline $23 F E B 85$ & 7.55 & 1.00 & 2.73 \\
\hline $25 \mathrm{FEB} 85$ & 0.73 & 0.70 & 0.68 \\
\hline $26 \mathrm{FEB} 85$ & 0.26 & . & 0.31 \\
\hline $27 \mathrm{FEB} 85$ & 0.25 & . & 0.18 \\
\hline $28 \mathrm{FEB} 85$ & 0.19 & . & 0.13 \\
\hline 01MAR85 & 0.25 & . & 0.10 \\
\hline 01MAR 85 & 0.34 & . & 0.09 \\
\hline 04MAR85 & 0.30 & . & 0.07 \\
\hline 05MAR 85 & 0.35 & . & . \\
\hline 06MAR8 5 & 0.27 & . & . \\
\hline 07 MAR85 & 0.23 & . & . \\
\hline O8MAR8 5 & 0.29 & . & . \\
\hline 11MAR 85 & 0.35 & . & . \\
\hline 13 MAR 85 & 0.13 & . & . \\
\hline 15MAR8 5 & 0.41 & . & . \\
\hline 18MAR8 5 & 0.54 & . & . \\
\hline 2OMAR85 & 0.08 & . & . \\
\hline 22MAR 85 & 0.08 & . & . \\
\hline 25MAR8 5 & 0.05 & . & . \\
\hline 27MAR8 5 & 0.05 & . & . \\
\hline 29MAR 85 & 0.02 & . & . \\
\hline 10APR8 5 & . & . & 0.04 \\
\hline 11APR85 & . & . & 0.04 \\
\hline 12APR8 5 & 0.11 & . & 0.04 \\
\hline 13APR 85 & 13.00 & . & 0.04 \\
\hline 14APR85 & 73.00 & . & 0.63 \\
\hline 15APR 85 & 142.00 & . & 2.75 \\
\hline 16APR85 & 175.00 & . & 5.51 \\
\hline 17APR 85 & 189.00 & . & 7.30 \\
\hline 18APR 85 & 178.00 & . & 7.39 \\
\hline 19APR 85 & 138.00 & . & 6.82 \\
\hline 21APR85 & 14.90 & . & 2.69 \\
\hline 22APR8 5 & 4.40 & . & 1.03 \\
\hline 23APR8 5 & 1.70 & . & 0.49 \\
\hline 24APR 85 & 1.08 & . & 0.29 \\
\hline 25APR85 & 0.40 & . & 0.18 \\
\hline 26APR85 & 0.22 & . & 0.12 \\
\hline 28AFR 85 & 0.16 & . & 0.07 \\
\hline
\end{tabular}




\begin{tabular}{|c|c|c|c|}
\hline $\begin{array}{l}\text { Sampling } \\
\text { Date }\end{array}$ & $\begin{array}{c}\text { Iodide } \\
\text { concentration } \\
(\mathrm{ppm})\end{array}$ & $\begin{array}{c}\text { Bromide } \\
\text { concentration } \\
\text { (ppm) }\end{array}$ & $\begin{array}{c}\text { Lithium } \\
\text { concentration } \\
\text { (ppm) }\end{array}$ \\
\hline 29APR85 & 0.14 & . & 0.06 \\
\hline 02MAY85 & 0.13 & . & . \\
\hline 08MAY85 & 0.33 & . & . \\
\hline 13JUL85 & 0.30 & . & 0.13 \\
\hline 15JUL85 & 0.66 & . & 0.10 \\
\hline 16JUL85 & 110.00 & . & 0.37 \\
\hline $17 \mathrm{JUL} 85$ & & . & 0.08 \\
\hline 17JUL85 & 172.00 & . & 6.50 \\
\hline 18JUL85 & 200.00 & . & 8.72 \\
\hline 19JUL85 & 178.00 & . & 7.21 \\
\hline 20JUL85 & 180.00 & . & 8.56 \\
\hline 21JUL85 & 55.00 & . & 6.01 \\
\hline 22JUL85 & 3.50 & . & 1.77 \\
\hline 23JUL85 & 0.91 & . & 0.55 \\
\hline 24JUL85 & 0.95 & . & 0.30 \\
\hline 25JUL85 & 0.52 & . & 0.19 \\
\hline 26JUL85 & & . & 0.12 \\
\hline
\end{tabular}




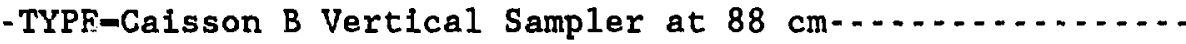

\begin{tabular}{|c|c|c|c|}
\hline $\begin{array}{l}\text { Sampling } \\
\text { Date }\end{array}$ & $\begin{array}{c}\text { Iodide } \\
\text { concentration } \\
\text { (ppm) }\end{array}$ & $\begin{array}{c}\text { Bromide } \\
\text { concentration } \\
\text { (ppm) }\end{array}$ & $\begin{array}{r}\text { Lithiv } \\
\text { concentra } \\
\text { (ppm) }\end{array}$ \\
\hline 15FEB85 & 106.00 & 48.13 & 2.79 \\
\hline 16FEB85 & 113.00 & 57.09 & 3.61 \\
\hline $18 \mathrm{FEB} 85$ & 120.00 & 73.00 & 4.47 \\
\hline 19FEB85 & 133.00 & . & 4.65 \\
\hline 20FEB85 & 116.00 & 63.00 & 4.88 \\
\hline $21 \mathrm{FEB} 85$ & 100.00 & 47.00 & 4.44 \\
\hline 22 FEB 85 & 74.90 & 32.00 & 3.60 \\
\hline 23 FEB 85 & 41.10 & 23.00 & 2.22 \\
\hline $25 F E B 85$ & 21.20 & 14.00 & 1.98 \\
\hline 26 FEB 85 & 11.10 & 7.00 & 1.68 \\
\hline 27 FEB 85 & 4.11 & 2.00 & 1.24 \\
\hline 28FEB 85 & 1.14 & 0.40 & 0.81 \\
\hline O1MAR85 & 0.18 & . & 0.49 \\
\hline O2MAR85 & 0.23 & . & 0.31 \\
\hline 04MAR85 & 0.28 & . & 0.14 \\
\hline 05MAR85 & 0.35 & . & 0.11 \\
\hline 06MAR85 & 0.19 & . & 0.09 \\
\hline 07MAR85 & 0.26 & . & . \\
\hline 08MAR85 & 0.27 & . & . \\
\hline 11MAR85 & 0.31 & . & . \\
\hline 12MAR85 & 0.13 & . & . \\
\hline 15MAR85 & 0.43 & . & . \\
\hline 18MAR85 & 0.54 & . & . \\
\hline 20MAR85 & 0.08 & . & . \\
\hline 22MAR85 & 0.05 & . & . \\
\hline 25MAR85 & 0.04 & . & . \\
\hline 27MAR85 & 0.04 & . & . \\
\hline 10APR85 & . & . & 0.04 \\
\hline 11APR 85 & . & . & 0.04 \\
\hline 12APR85 & 0.11 & . & 0.04 \\
\hline 13APR85 & 6.40 & . & 0.04 \\
\hline 14APR 85 & 48.00 & . & 0.55 \\
\hline 15APR85 & 75.00 & . & 1.86 \\
\hline 16APR85 & 108.00 & . & 3.10 \\
\hline 17APR85 & 126.00 & . & 3.88 \\
\hline 18APR85 & 137.00 & . & 4.47 \\
\hline 19APR85 & 132.00 & . & 4.95 \\
\hline 21APR85 & 63.00 & . & 3.70 \\
\hline 22APR 85 & 45.00 & . & 2.98 \\
\hline 23APR 85 & 32.00 & . & 2.43 \\
\hline 24APR 85 & 20.70 & . & 2.07 \\
\hline 25APR8 5 & 6.00 & . & 1.49 \\
\hline 26APR 85 & 1.56 & . & 0.77 \\
\hline 28APR 85 & 0.49 & . & 0.23 \\
\hline 29APR 85 & 0.19 & . & 0.04 \\
\hline
\end{tabular}


- TYPE=Caisson B Vertical Sampler at 88

\begin{tabular}{|c|c|c|c|}
\hline $\begin{array}{l}\text { Sampling } \\
\text { Datee }\end{array}$ & $\begin{array}{c}\text { Iodide } \\
\text { concentration } \\
\text { (ppm) }\end{array}$ & $\begin{array}{c}\text { Bromide } \\
\text { concentration } \\
\text { (ppm) }\end{array}$ & $\begin{array}{c}\text { Lithium } \\
\text { concentration } \\
\text { (ppm) }\end{array}$ \\
\hline O2MAY85 & 0.15 & . & 0.12 \\
\hline 08MAY85 & 0.16 & . & 0.12 \\
\hline 15MAY85 & 0.21 & . & \\
\hline 13JUL.85 & 0.37 & . & 0.13 \\
\hline $15 \mathrm{JUL} 85$ & 0.11 & . & 0.09 \\
\hline $16 \mathrm{JUL} 85$ & 5.40 & . & 0.08 \\
\hline 17JUL85 & 70.00 & . & 0.87 \\
\hline 18JUL85 & 114.00 & . & 2.80 \\
\hline 19 JUL85 & 166.00 & . & 4.35 \\
\hline 20JTI85 & 170.00 & . & 7.21 \\
\hline 21JUL85 & 140.00 & . & 7.60 \\
\hline 22JULL85 & 79.00 & . & 5.99 \\
\hline $23 \mathrm{JULZ} 85$ & 39.00 & . & 3.86 \\
\hline 24JUL85 & 9.30 & . & 2.23 \\
\hline 25.JUL85 & 2.40 & . & 1.08 \\
\hline 26JUI85 & 0.57 & . & 0.47 \\
\hline 27JUL85 & 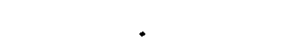 & . & 0.25 \\
\hline 28JUL85 & & . & 0.16 \\
\hline
\end{tabular}


TYPE-Caisson B Vertical Sampler at 103

\begin{tabular}{|c|c|c|c|}
\hline $\begin{array}{l}\text { Sampling } \\
\text { Date }\end{array}$ & $\begin{array}{c}\text { lodide } \\
\text { concentration } \\
(\text { ppm })\end{array}$ & $\begin{array}{c}\text { Bromide } \\
\text { concentration } \\
\text { (ppm) }\end{array}$ & $\begin{array}{r}\text { Lithi } \\
\text { concentr } \\
\text { (ppm }\end{array}$ \\
\hline $15 F E B 85$ & 60.20 & 18.4 & 0.14 \\
\hline $16 \mathrm{FEB} 85$ & 68.00 & • & 1.03 \\
\hline 18FEB85 & 79.00 & 49.0 & 2.06 \\
\hline 19FEB85 & 91.00 & • & 2.64 \\
\hline 20FEB85 & 101.00 & 58.0 & 2.97 \\
\hline 21FEB85 & 104.00 & 50.0 & 3.18 \\
\hline 22FEB8 5 & 78.20 & 38.0 & 2.97 \\
\hline 23FEB85 & 55.80 & 33.0 & 2.47 \\
\hline 25FEB85 & 58.70 & 40.0 & 1.95 \\
\hline $26 \mathrm{FEB} 85$ & 51.50 & 35.0 & 1.84 \\
\hline 27FEB85 & 60.90 & 25.0 & 1.95 \\
\hline 28FEB85 & 44.20 & 21.0 & 2.11 \\
\hline OIMAR85 & 32.60 & 20.0 & 2.05 \\
\hline O2MAR85 & 25.00 & 13.0 & 2.01 \\
\hline 04 MAR8 5 & 10.20 & 4.0 & 1.68 \\
\hline 05MAR 85 & 3.09 & 1.0 & 1.16 \\
\hline 06MAR8 5 & 0.72 & . & 0.83 \\
\hline 07MAR85 & 0.12 & . & • \\
\hline 08MAR85 & 0.18 & . & . \\
\hline 11MAR85 & 0.27 & . & . \\
\hline 13MAR85 & 0.08 & . & . \\
\hline 15MAR85 & 0.39 & . & . \\
\hline 18MAR85 & 0.52 & . & . \\
\hline 20MAR85 & 0.08 & . & - \\
\hline 22MAR85 & 0.06 & . & . \\
\hline 25MAR85 & 0.05 & . & . \\
\hline 27MAR85 & 0.05 & . & • \\
\hline 10APR85 & . & . & 0.04 \\
\hline 11APR85 & . & . & 3.47 \\
\hline 12APR 85 & . & . & 0.04 \\
\hline 13APR85 & . & . & 0.04 \\
\hline 14APR8 5 & 0.04 & . & 0.04 \\
\hline 15APR85 & . & . & 0.04 \\
\hline 16APR85 & 14.30 & . & 0.04 \\
\hline 17APR85 & 34.00 & . & 0.25 \\
\hline 18APR85 & 54.00 & . & 0.80 \\
\hline 19APR85 & 74.00 & . & 1.49 \\
\hline 21APR85 & 92.00 & . & 2.64 \\
\hline 22APR85 & 93.00 & . & 2.93 \\
\hline 23APR85 & 87.00 & . & 2.91 \\
\hline 24APR85 & 79.00 & . & 2.90 \\
\hline 25APR 85 & 69.00 & . & 2.80 \\
\hline 26APR85 & 55.00 & - & 2.82 \\
\hline 28APR85 & 49.00 & - & 3.07 \\
\hline 29APR85 & 24.00 & - & 2.73 \\
\hline
\end{tabular}




$\begin{array}{cccc}\begin{array}{c}\text { Sampling } \\ \text { Date }\end{array} & \begin{array}{c}\text { Iodide } \\ \text { concentration } \\ \text { (ppm) }\end{array} & \begin{array}{c}\text { Bromide } \\ \text { concentration } \\ \text { (ppm) }\end{array} & \begin{array}{c}\text { Lithium } \\ \text { concentration } \\ \text { (ppin) }\end{array} \\ \text { 02MAY85 } & 10.00 & . & 2.71 \\ \text { 08MAY85 } & 1.50 & . & 1.37 \\ \text { 15MAY85 } & 0.55 & . & 0.67 \\ \text { 13JUL85 } & 0.19 & . & 0.19 \\ \text { 15JUL85 } & 0.16 & . & 0.16 \\ \text { 16JUL85 } & 1.05 & . & 0.20 \\ \text { 17JUL85 } & 1.81 & . & 0.16 \\ \text { 18JUL85 } & 18.00 & . & 0.19 \\ \text { 19JUL85 } & 73.00 & . & 1.11 \\ \text { 20JUL85 } & 113.00 & . & 2.94 \\ \text { 21JUL85 } & 132.00 & . & 3.50 \\ \text { 22JUL85 } & 150.00 & . & 4.81 \\ \text { 23JUL85 } & 131.00 & . & 5.31 \\ \text { 24JUL85 } & 100.00 & . & 5.80 \\ \text { 25JUL85 } & 81.00 & . & 5.60 \\ \text { 26JUL85 } & 47.00 & . & 4.21 \\ \text { 27JUL85 } & . & . & 3.50 \\ \text { 28JUL85 } & . & . & 2.40 \\ \text { 29JUL85 } & . & . & 1.38 \\ \text { 30JUL85 } & . & . & 0.71 \\ \text { 01AUG85 } & . & . & 0.28 \\ \text { 04AUG85 } & . & & 0.12\end{array}$


-TYPE-Caisson B Vertical Sampler at $118 \mathrm{~cm} \ldots \ldots . . . . . . . . .$.

\begin{tabular}{|c|c|c|c|}
\hline $\begin{array}{l}\text { Sampling } \\
\text { Date }\end{array}$ & $\begin{array}{c}\text { Iodide } \\
\text { concentration } \\
\text { (ppm) }\end{array}$ & $\begin{array}{c}\text { Bromide } \\
\text { concentration } \\
\text { (ppm) }\end{array}$ & $\begin{array}{c}\text { Lithium } \\
\text { concentration } \\
\text { (ppm) }\end{array}$ \\
\hline 06FEB85 & 7.10 & 4.38 & 0.04 \\
\hline 08FEB85 & 93.00 & 58.00 & 1.55 \\
\hline 15FEB85 & 0.03 & 1.30 & 0.04 \\
\hline 19FEB85 & 120.00 & . & 2.58 \\
\hline 20FEB85 & 132.00 & 70.00 & 3.69 \\
\hline 21FEB85 & 136.00 & 67.00 & 4.10 \\
\hline 22FEB8 5 & 118.00 & 65.00 & $\cdot$ \\
\hline $25 F E B 85$ & 43.40 & 31.00 & 2.95 \\
\hline 26FEB85 & 37.60 & 26.00 & 2.35 \\
\hline $27 \mathrm{FEB} 85$ & 28.50 & 22.00 & 1.86 \\
\hline $28 \mathrm{FEB} 85$ & 37.30 & 20.00 & 1.71 \\
\hline 28FEB85 & 81.10 & 52.00 & 4.19 \\
\hline 01MAR85 & 31.60 & 22.00 & 1.46 \\
\hline 02MAR85 & 25.30 & 17.00 & 1.35 \\
\hline 04MAR85 & 20.30 & 7.00 & 1.34 \\
\hline 05MAR85 & 14.90 & 6.00 & 1.37 \\
\hline 06MAR85 & 9.60 & 2.00 & 1.31 \\
\hline 07MAR85 & 6.32 & 3.00 & 1.27 \\
\hline 08MAR85 & 3.59 & 2.00 & 1.16 \\
\hline 11MAR85 & 0.47 & · & 0.70 \\
\hline 12MAR85 & 0.10 & . & • \\
\hline 15MAR85 & 0.31 & . & . \\
\hline 18MAR85 & 0.46 & . & . \\
\hline 20MAR85 & 0.20 & . & . \\
\hline 22MAR85 & 0.09 & . & . \\
\hline 25MAR85 & 0.09 & . & . \\
\hline 27MAR85 & 0.08 & . & . \\
\hline 10APR85 & . & . & 0.04 \\
\hline 13APR85 & . & . & 0.04 \\
\hline 14 APP 5 & • & . & 0.04 \\
\hline 15APR85 & 0.04 & . & 0.04 \\
\hline 16APR85 & 0.29 & . & 0.04 \\
\hline 17APR85 & 17.20 & . & 0.04 \\
\hline 18APR85 & 48.00 & . & 0.09 \\
\hline 19APR85 & 87.00 & . & 1.37 \\
\hline 21APR85 & 122.00 & . & 3.46 \\
\hline 22APR85 & 121.00 & . & 3.75 \\
\hline 23APR85 & 93.00 & . & 4.21 \\
\hline 24APR85 & 68.00 & . & 3.83 \\
\hline 25APR85 & 47.00 & . & 3.27 \\
\hline 26APR85 & 36.00 & . & 2.73 \\
\hline 28APR85 & 22.00 & . & 1.72 \\
\hline 29APR85 & 17.00 & . & 1.61 \\
\hline 02MAY85 & 12.90 & - & 1.45 \\
\hline 08MAY85 & 6.30 & . & 1.05 \\
\hline
\end{tabular}


-TYPE-Caisson B Vertical Sampler at 118

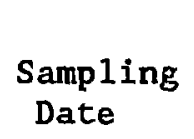

15MAY85

14JUL85

$16 J U L 85$

17 JUL85

18JUL85

19JUL85

20JUL85

21JUL85

22JUL85

23JUL85

24JUL85

25JUL85

26JUL85

27JUL85

28JUL85

29JUL85

30JUL85

01AUG85

02AUG85

04AUG85

\section{Iodide \\ concentration (ppm)}

4.50
0.45
0.22
0.84
3.10
12.60
97.00
132.00
120.00
148.00
129.00
85.00
46.00
.
.
.

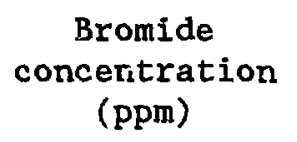

Lithium
concentration
(ppm)

0.88

0.34

0.22

0.30

0.30

0.63

3.40

4.05

5.00

5.89

5.96

4.12

3.35

2.63

2.05

1.54

0.69

0.41

0.19 


$\begin{array}{ll}\text { Sampling } & \begin{array}{l}\text { Iodide } \\ \text { Concentration } \\ \text { Date }\end{array} \\ \text { (ppm) }\end{array}$

\section{Bromide Lithium Concentration Concentration (ppm) (ppm)}

\begin{tabular}{|c|c|}
\hline 30JUL84 & \\
\hline 01AUG84 & 0.02 \\
\hline 02AUG84 & 0.02 \\
\hline 03AUG84 & • \\
\hline 06AUG84 & . \\
\hline 07AUG84 & . \\
\hline 08AUG84 & . \\
\hline 09AUG84 & . \\
\hline 10AUG84 & . \\
\hline 11AUG84 & . \\
\hline 12AUG84 & . \\
\hline 13AUG84 & . \\
\hline 19AUG84 & . \\
\hline 20AUG84 & . \\
\hline 21AUG84 & . \\
\hline 26AUG84 & . \\
\hline 04SEP84 & \\
\hline 06SEP84 & 0.04 \\
\hline 07SEP84 & . \\
\hline 11SEP84 & . \\
\hline 14SEP84 & \\
\hline $050 \mathrm{CT} 84$ & 0.04 \\
\hline $100 \mathrm{CT} 84$ & - \\
\hline $120 \mathrm{CT} 84$ & . \\
\hline $150 \mathrm{CT} 84$ & . \\
\hline $170 \mathrm{CT} 84$ & . \\
\hline 190CT84 & . \\
\hline $230 \mathrm{CT} 84$ & . \\
\hline $240 \mathrm{CT} 84$ & . \\
\hline $250 \mathrm{CT} 84$ & $\cdot$ \\
\hline $290 \mathrm{CT} 84$ & 0.08 \\
\hline $300 \mathrm{CT} 84$ & 1.95 \\
\hline $310 \mathrm{CT} 84$ & 3.60 \\
\hline 02NOV84 & 3.80 \\
\hline 05NOV84 & 3.60 \\
\hline 07NOV84 & 3.30 \\
\hline 09NOV84 & 0.78 \\
\hline 12NOV84 & 6.40 \\
\hline 14 NOV84 & 8.80 \\
\hline 16NOV84 & 8.80 \\
\hline 19NOV84 & 8.70 \\
\hline 21NOV84 & 13.60 \\
\hline 23NOV84 & 10.30 \\
\hline 26NOV84 & 22.00 \\
\hline 28NOV84 & 15.20 \\
\hline
\end{tabular}

\subsection{5}

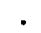

•

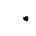

.

.

$$
\text { . }
$$$$
\text { . }
$$$$
\text { . }
$$$$
\cdot
$$$$
\cdot
$$$$
\cdot
$$$$
\text { . }
$$$$
\text { . }
$$$$
0.020
$$$$
0.020
$$

0.218

0.020

0.020

0.020

0.020

0.020

0.020

0.092

0.020

0.020

0.548

1. 218

2.090

2. 312

2. 216

1.805

2.083

4.898

6.439

7.467

9.516

12.407

16.250

15.920

13.468
0.020

0.020

0.013

0.010

0.020

0.020

0.020

0.020

0.020

0.020

0.020

0.020

0.020

0.020

0.020

.

0.040

0.040

0.040

0.040

0.040

0.040

0.040

0.080

0.090

0.100

0.110

0.110

0.120

0.170

0.200

0.240

0.340

0.370 


\begin{tabular}{|c|c|c|c|}
\hline $\begin{array}{l}\text { Sampling } \\
\text { Date }\end{array}$ & $\begin{array}{l}\text { Iodide } \\
\text { Concentration } \\
\text { (ppm) }\end{array}$ & $\begin{array}{l}\text { Bromide } \\
\text { Concentration } \\
\text { (ppm) }\end{array}$ & $\begin{array}{l}\text { Lithium } \\
\text { Concentration } \\
\text { (ppm) }\end{array}$ \\
\hline 30 NOV84 & 5.60 & 7.465 & 0.40 \\
\hline 03DEC84 & 2.80 & 2.456 & 0.40 \\
\hline 04DEC84 & 2.40 & 2.089 & . \\
\hline 05DEC84 & 0.80 & 1.032 & 0.82 \\
\hline 06DEC84 & 0.80 & 0.833 & . \\
\hline 07DEC84 & 0.17 & 0.462 & . \\
\hline 08DEC84 & 0.29 & 0.412 & 0.85 \\
\hline 09DEC84 & 0.09 & 0.736 & 0.78 \\
\hline 10DEC84 & 0.09 & 0.467 & 0.72 \\
\hline 11DEC84 & 0.04 & 0.441 & . \\
\hline 12DEC84 & 0.10 & 0.325 & . \\
\hline 13DEC84 & . & 0.283 & . \\
\hline 14DEC84 & . & 0.225 & . \\
\hline $15 \mathrm{DEC} 84$ & 0.28 & 0.281 & . \\
\hline 17DEC84 & 0.09 & 0.196 & . \\
\hline 18DEC84 & 0.15 & 0.132 & . \\
\hline 19DEC84 & 0.14 & 0.135 & . \\
\hline 20DEC84 & 0.16 & • & . \\
\hline $21 D E C 84$ & 0.15 & . & . \\
\hline 22DEC84 & 0.12 & . & . \\
\hline 24DEC84 & 0.07 & . & . \\
\hline 26DEC84 & 0.08 & . & . \\
\hline 27DEC84 & 0.07 & . & . \\
\hline 28DEC84 & 0.17 & . & . \\
\hline 29DEC84 & 0.06 & . & . \\
\hline $31 D E C 84$ & 1.90 & . & . \\
\hline 02JAN85 & 10.10 & . & . \\
\hline 03JAN85 & 15.90 & $\dot{.}$ & . \\
\hline 04JAN85 & 22.10 & . & . \\
\hline 05JAN85 & 28.30 & . & . \\
\hline 07JAN85 & 36.00 & . & . \\
\hline O8JAN85 & 35.00 & . & . \\
\hline 09JAN85 & 33.10 & . & . \\
\hline 10JAN85 & 33.00 & . & . \\
\hline 11JAN85 & 35.00 & . & . \\
\hline 12JAN85 & 27.70 & . & . \\
\hline 14JAN85 & 25.30 & . & . \\
\hline 16JAN85 & 22.20 & . & . \\
\hline 18JAN85 & 17.70 & . & . \\
\hline 21JAN85 & 14.28 & . & . \\
\hline 23JAN85 & 17.50 & . & . \\
\hline 25JAN85 & 26.10 & . & . \\
\hline 28JAN85 & 27.90 & . & . \\
\hline 30JAN85 & 23.80 & . & . \\
\hline 01FEB85 & 7.41 & . & . \\
\hline
\end{tabular}




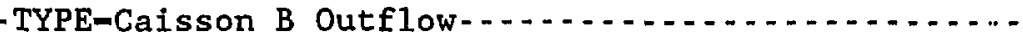

$\begin{array}{llll} & \text { Iodide } & \text { Bromide } & \text { Lithium } \\ \text { Sampling } & \text { Concentration } & \text { Concentration } & \text { Concentration } \\ \text { Date } & (\mathrm{ppm}) & (\mathrm{ppm}) & \text { (ppm) }\end{array}$

04FEB85

06FEB85

08FEB85

09FEB85

10FEB85

11 FEB 85

5.20

5.20

3.67

3.38

2.53

2.83

1.22

0.75

0.69

13FEB 85

14FEB85

0.53

15 FEB 85

16FEB8 5

18FEB85

0.34

0.23

$19 F E B 85$

20FEB85

21FEB85

22FEB85

23FEB85

25FEB85

26FEB85

27FEB85

28FEB85

01MAR85

02MAR85

04MAR 85

05MAR85

06MAR85

07MAR85

08MAR8 5

11MAR85

13MAR85

15MAR 85

18 MAR 85

20MAR 85

22MAR85

25MAR8 5

27MAR 85

29MAR8 5

01APR 85

0.19

0.19

0.37

0.16

0.13

0.11

0.11

0.11

0.32

0.32

0.28

0.46

3.80

10.60

16.30

22.50

40.50

35.30

33.00

26.20

23.40

23.90

21.30

22.20

32.00

36.00

03APR8 5

27.00

17.00

6.40

5.40

$$
\begin{aligned}
& 2.713 \\
& 2.094 \\
& 1.874 \\
& 1.494
\end{aligned}
$$

OSAPR85

09APR85

10APR85

15APR85

21APR85 


$\begin{array}{llll} & \text { Iodide } & \begin{array}{l}\text { Bromide } \\ \text { Concentration }\end{array} & \begin{array}{l}\text { Lithium } \\ \text { Concentration } \\ \text { Sampling }\end{array} \\ \text { Concentration } & \begin{array}{l}\text { (ppm) } \\ \text { (ppm) }\end{array} & \text { (ppm) }\end{array}$

\begin{tabular}{|c|c|}
\hline 22APR85 & 0.11 \\
\hline 23APR 85 & 0.09 \\
\hline 24APR85 & 0.10 \\
\hline 25APR85 & 0.25 \\
\hline 26APR85 & 0.04 \\
\hline 28APR85 & 4.50 \\
\hline 29APR85 & 4.10 \\
\hline 2OMAY85 & 11.60 \\
\hline 21MAY85 & 16.70 \\
\hline 22MAY85 & 16.00 \\
\hline 23MAY85 & 12.00 \\
\hline 24MAY85 & 17.20 \\
\hline 28MAY85 & 16.50 \\
\hline 30MAY85 & 16.80 \\
\hline 04JUN85 & 10.00 \\
\hline 13JUL85 & \\
\hline 13JUL85 & 20.50 \\
\hline 14JUL85 & \\
\hline 14JUL85 & 20.40 \\
\hline 15JUL85 & 13.00 \\
\hline 16JUL85 & 16.80 \\
\hline 17JUL85 & \\
\hline 17JUL85 & 17.30 \\
\hline 18JUL85 & 18.30 \\
\hline 19JUL85 & \\
\hline 19JUL85 & 17.00 \\
\hline 20JUL85 & 18.00 \\
\hline 21JUL85 & . \\
\hline 21JUL85 & 18.00 \\
\hline 22JUL85 & 10.30 \\
\hline 23JUL85 & ${ }^{\circ}$ \\
\hline 23JUL85 & 9.10 \\
\hline 24JUL85 & 19.70 \\
\hline 25JUL85 & 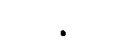 \\
\hline 25JUL85 & 23.80 \\
\hline 26JUL85 & 25.00 \\
\hline 27JUL85 & 42.00 \\
\hline 28JUL85 & 54.00 \\
\hline 29JUL85 & 50.00 \\
\hline 01AUG85 & 35.00 \\
\hline 02AUG85 & 34.00 \\
\hline 04AUG85 & 30.00 \\
\hline 05AUG85 & 33.00 \\
\hline 07AUG85 & 39.00 \\
\hline 09AUG85 & 50.00 \\
\hline
\end{tabular}

0.11

0.09

0.10

0.25

0.04

4.50

4.10

11.60

16.70

16.00

12.00

17.20

16.50

16.80

0.50

20.40

3.00

7.30

8.30

17.00

8.00

. 10

19.70

3.80

25.00

2.00

4.00

0.00

5.00

4.00

0.00

3.00

50.00 (ppm)

0.35

0.25

0.30

0.64

0.54

0.52

0.54

0.51

0.54

0.48

0.53

0.55

1.49

0.64

.

0.47

0.93

0.45

.

0.55

.

0.58

1.03

1.42

1.54

1.40 


Samplin
Date
12AUG85
14AUG85
16AUG85
19AUG85
21AUG85
23AUG85
28AUG85

Iodide

Concentration

(ppm)

41.0

56.0

46.0

34.0

23.6

21.5

\section{Bromide \\ Concentration (ppm)}

\section{Lithium \\ Concentration (ppm)}

1.56

2.32

2.86

2.48

1.96 